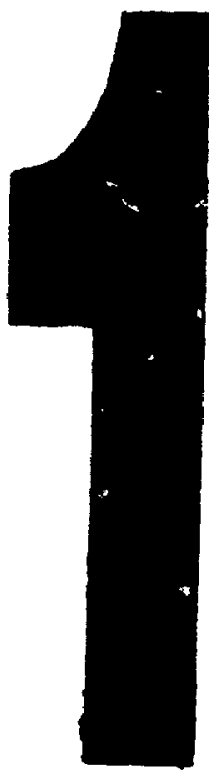

PM-1 31\%" $\times 4$ " PHOTOGRAPHIC MICROCOPY TARGET NBS 1010a ANSI/ISO *2 EQUIVALENT

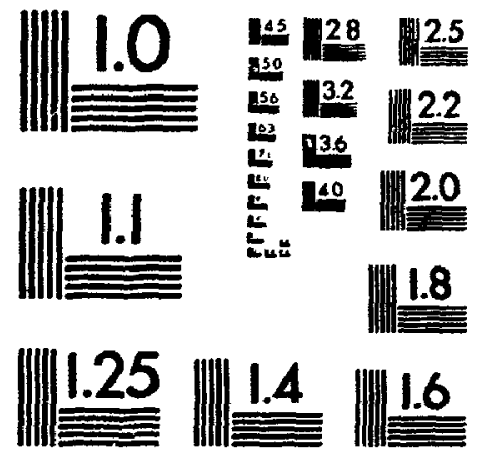

PRECISIONSM RESOLUTION TARGETS 
National Library

of Canada

Acquisitions and

Bibliographic Services Branch

395 Wellington Street

Ottawa. Onlario

K1A ONA
Bibliothèquc nationale

du Canada

Direction des acquis cions et

des services bibliry raphiquos

395, rue Wellington

Otawa (Ontario) hour the volvesterence

Our fite Notre reteremice

NOTICE

AVIS

The quality of this microform is heavily dependent upon the quality of the original thesis submitted for microfilming. Every effort has been made to ensure the highest quality of reproduction nossible.

If pages are missing, contact the university which granted the degree.

Some pages may have indistinct print especially if the original pages were typed with a poor typewriter ribbon or if the university sent us an inferior photocopy.

Reproduction in full or in part of this microform is governed by the Canadian Copyright Act, R.S.C. 1970, c. C-30, and subsequent amendments.
La qualité de cette microforme dépend grandement de la qualité de la thèse soumise au microfilmage. Nous avons tout fait pour assurer une qualité supérieure de reproduction.

S'il manque des pages, veuillez communiquer avec l'université qui a conféré le grade.

La qualité d'impression de certaines pages peut laisser à désirer, surtout si les pages originales ont été dactylographiées à l'aide d'un ruljan usé ou si l'université nous a iait parvenir une photocopie de qualité inférieure.

La reproduction, même partielle, de cette microforme est soumise à la Loi canadienne sur le droit N'auteur, SRC 1970, c. C-30, et ses amendements subséquents.

\section{Canadä}




\title{
Nucleotide Pattern Matching on the Intel iPSC/860 Hypercube
}

\author{
by \\ John L. Houle, B.Sc., Hons. B.Sc. \\ A thesis submitted to \\ the Faculty of Graduate Studies and Research \\ in partial fulfilment of \\ the requirements for the degree of \\ Master of Computer Science
}

Ottawa-Carleton Institute for Computer Science

School of Computer Science

Carleton University

Ottawa, Ontario

December 1, 1994

Copyright (C) 1994, John L. Houle, B.Sc., Hons. B.Sc. 
National Library

of Canada

Acquisitions and Bibliographic Services Branch

395 Wellingion Street Ottawa, Ontario

KIA ON4
Bibliotheque nationale du Canada

Direction des acquisitions et des services bibliographiques

395, nue Wellington

Ottawa (Ontario)

K1A ONA
Your tis votre noturence

Our in Nolre newrence
THE AUTHOR HAS GRANTED AN IRREVOCABLE NON-EXCLUSIVE LICENCE ALLOWING THE NATIONAL LIBRARY OF CANADA TO REPRODUCE, LOAN, DISTRIBUTE OR SELL COPIES OF HIS/HER THESIS BY ANY MEANS AND IN ANY FORM OR FORMAT, MAKING THIS THESIS AVAII ABLE TO INTERESTED PERSONS.
L'AUTEUR A ACCORDE UNE LICENCE IRREVOCABLE ET NON EXCLUSIVE PERMETTANT A LA BIBLIOTHEQUE NATIONALE DU CANADA DE REPRODUIRE, PRETER, DISTRIBUER OU VENDRE DES COPIES DE SA THESE DE QUELQUE MANIERE ET SOUS QUELQUE FORME QUE CE SOIT POUR METTRE DES EXEMPLAIRES DE CETTE THESE A LA DISPOSITION DES PERSONNE INTERESSEES.
THE AUTHOR RETAINS OWNERSHIP OF THE COPYRIGHT IN HIS/HER THESIS. NEITHER THE THESIS NOR SUBSTANTIAL EXTRACTS FROM IT MAY BE PRINTED OR OTHERWISE REPRODUCED WITHOUT HIS/HER PERMISSION.
L'AUTEUR CONSERVE LA PROPRIETE DU DROIT D'AUTEUR QUI PROTEGE SA THESE. NI LA THESE NI DES EXTRAITS SUBSTANTIELS DE CELLECI NE DOIVENT ETRE IMPRIMES OU AUTREMENT REPRODUITS SANS SON AUTORISATION.

ISBN $\quad 0-315-98567-4$ 
The undersigned recommend to the Faculty of Graduate Studies and Research acceptance of the thesis

Nucleotide Pattern Matching on the Intel iPSC/860 Hypercube submitted by

John L. Houle, B.Sc., Hons. B.Sc.

in partial fulfillment of the requirements for the degree of Master of Computer Science

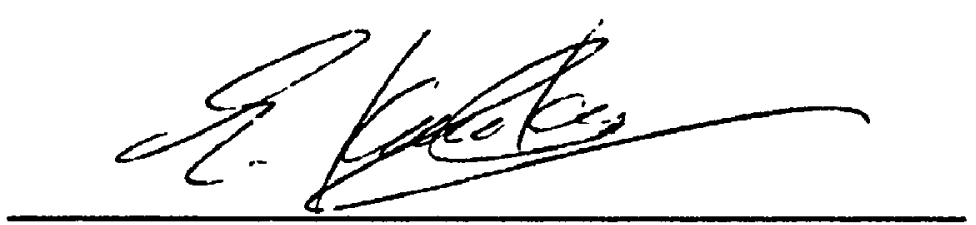

Chairman, School of Computer Science

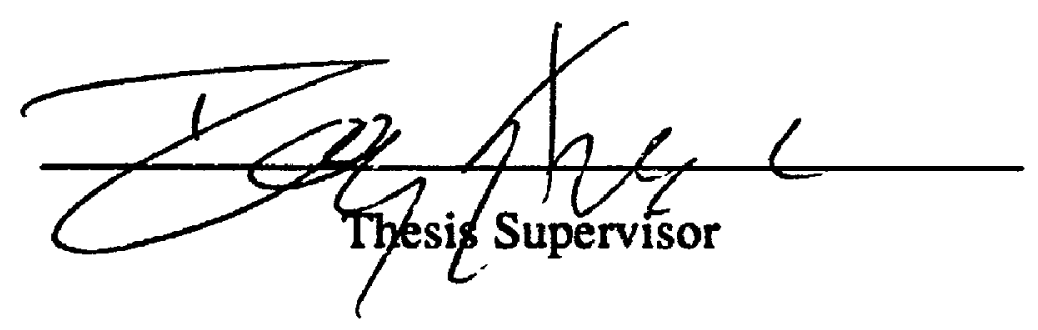

Carleton University

December 1, 1994 


\section{Abstract}

Numerous sequence analysis problems in computational molecular biology can be expressed as string search problems. We consider the multiple keyword search problem and develop a parallel solution for the hypercube architecture. The thesis reports a multiple keyword search algorithm for the Intel iPSC/860 Hypercube. The data-parallel algorithm is specialized to searching for class 1 nucleotide motifs within DNA sequences. The human interferon-alpha multigene family within the 9p13 - 9p22 chromosomal bands was characterized using restriction analysis and transcription analysis. Using this algorithm we have produced a series of human interferon-alpha restriction maps and transcription maps. Transcription analysis revealed five families of potential promoter elements: 1. tata boxes, 2. virus-response elements, 3. distal interferon regulatory factor sites, 4, proximal interferon regulatory factor sites and 5. TG sequences. On the basis of computational evidence, a theoretical model for the transcriptional control of human interferon-alpha gene expression is proposed. Future directions for human interferon-alpha transcriptional regulation research are identified. 


\section{Acknowledgements}

The author acknowledges the contributions of several individuals and institutions. The systematic characterization of the human interferon-alpha promoters was a problem originally posed to us by Dr. Mark Hayes of the Division of Cytokine Biology at the Center for Biologics Evaluation and Research, Food and Drug Administration.

Theoretical aspects of algorithm design were developed in collaboration with Dr. Elena Lodi and Dr. Nicola Santoro. Dr. Elena Lodi was a visiting researcher from the Department of Mathematics at the University of Siena. Dr. Nicola Santoro is a professor with the School of Computer Science at Carleton University. Dr. Nicola Santoro was my thesis advisor. I am particularly indebted to Dr. Santoro for his academic and financial support throughout the duration of my masters candidacy. Together we experimented with several strategies for solving the multiple keyword search problem in nucleotide sequences. These brainstorming sessions formed an integral coniponent of graduate education.

The mechanics of hypercube operation were assisted by Anton Saarimaki and Peter Choynowski. Masters candidate Anton Saarimaki provided a wealth of experience in the correct use of hypercube parallel library routines. Software engineer Peter Choynowski maintained the operation of the Intel iPSC/860 Hypercube.

Sequence data was generously provided by Dana Macelis and the National Center for Biotechnology Information. Dana Macelis of New England BioLabs sent several copies of REBASE, the restriction endonuclease database. The National Center for Biotechnology Information at the National Library of Medicine, National Institutes of Health sent a copy of TFD, the transcription factors database. The Genbank molecular sequence database of the National Center for Biotechnology Infomation sent further copies of the human retinoblastoma susceptibility gene and the human interferon-alpha genes. 
Scientific visualization of molecular biology data was made possible through the efforts of Dr. Paul Adler, Dr. Manual Diaz, Dr. Nicholas Murgolo, Nancy Manning, Govindachari Raghunath, Blair Griezic and Stacy Fietz. Dr. Paul Adler of the Department of Pathology at the University of Washington generated the human chromosome 9 ideogram. Dr. Manual Diaz of the Oncology Institute at the Loyola University Medical Center conveyed a preliminary copy of the human type-I interferon gene cluster map. Dr. Nicholas Murgolo of Schering-Plough Pharmaceuticals released x-ray crystallographic coordinates of the human interferon-alpha 2 protein which were modeled by Nancy Manning of the Protein Data Bank at the Brookhaven National Laboratory. Doctoral candidate Guvindachari Raghunath provided instruction in the use of both the CricketGraph graph plotter and the Hewlett-Packard ScanJet Ilex image scanner. Graphic artists Blair Griezic and Stacy Fietz drew all the genetic maps.

This research was supported by National Science and Engineering Research Council research grant \#A2415. 


\section{Contents}

Abstract

Acknowledgements iv

List of Tables $\quad x i$

List of Figures $\quad x i v$

1 Introduction 1

1.1 Genome Informatics 1

1.2 Nucleotide Motifs $\quad 8$

$\begin{array}{ll}1.3 \text { Notation and Problem Statement } & 10\end{array}$

1.4 Thesis Contributions $\quad 12$

$\begin{array}{ll}1.5 \text { Thesis Outline } & 14\end{array}$

2 Moiecular Biology 15

2.1 Nucleic Acids 16

2.2 Protein 26

2.3 Restriction Endonucleases 34

2.4 Transcription Factors 38

2.5 Interferon 42

2.6 Retinoblastoma 49

2.7 Human Genome Project 51 
3 Nucleotide Motif Search Algorithm 55

3.1 Keyword Search Problem 55

3.2 Nucleotide Motif Search Problem 56

3.3 Nucleotide Motif Search Algorithm 58

4 Intel iPSC/860 Hypercube Implementation 60

4.1 Hypercube Network $\quad 60$

4.2 Data-Parallel Programming Model 62

4.3 Intel iPSC/860 Hypercube Implementation 64

5 Experimental Results $\quad 66$

5.1 Human Interferon-Alpha 1 Gene 71

5.2 Human Interferon-Alpha 2 Gene 72

5.3 Human Interferon-Alpha 4 Gene 73

5.4 Human Interferon-Alpha 5 Gene 74

5.5 Human Interferon-Alpha 6 Gene 75

5.6 Human Interferon-Alpha 7 Gene 76

5.7 Human Interferon-Alpha 8 Gene 77

5.8 Human Interferon-Alpha-13 Gene $\quad 78$

5.9 Human Interferon-Alpha 14 Gene 79

5.10 Human Interferon-Alpha 16 Gene 80

5.11 Human Interferon-Alpha 17 Gene 81

5.12 Human Retinoblastoma Susceptibility Gene 82 
6 Genetic Analysis $\quad 83$

6.1 Human Interferon-Alpha Genetic Maps 83

6.2 Human Interferon-Alpha Promoter Transcription Maps 95

6.3 Human Interferon-Alpha Promoter Elements 101

6.4 Human Incerteron-Alpha Transcription Regulatory

Mechanism

106

7 Parallel Performance Analysis 110

7.1 Performance Measures 110

7.2 Restriction Endonucleases 112

7.3 Transcription Factors 115

7.4 Human Interferon Transcription Factors 118

$\begin{array}{ll}7.5 \text { Performance Analysis } & 121\end{array}$

8 Conclusions 123

8.1 Summary 123

$\begin{array}{ll}\text { 8.2 Future Research } & 126\end{array}$

$\begin{array}{lr}\text { A Restriction Endonucleases } & 129\end{array}$

B Transcription Factors 133

C Human Interferon Transcription Factors 174 
D Human Interferon-Alpha 1 Gene

D.1 Nucleotide Sequence

D.2 Restriction Endonuclease Sites

E Human Interferon-Alpha 2 Gene

E.1 Nucleotide Sequence

E.2 Restriction Endonuclease Sites

F Human Interferon-Alpha 4 Gene

F.1 Nucleotide Sequence

205

F.2 Restriction Endonuclease Sites

206

G Human Interferon-Alpha 5 Gene

221

G.1 Nucleotide Sequence

221

G.2 Restriction Endonuclease Sites

222

H Human Interferon-Alpha 6 Gene

H.1 Nucleotide Sequence

233

H.2 Restriction Endonuclease Sites

234

I Human Interferon-AIpha 7 Gene

I.1 Nucleotide Sequence

1.2 Restriction Endonuclease Sites 
J Human Interferon-Alpha 8 Gene 261

J.1 Nucleotide Sequence 261

J.2 Restriction Endonuclease Sites 262

K Human interferon-Alpha 13 Gene 271

K.1 Nucleotide Seq snce 271

K.2 Resrriction Endonuclease Sites 272

L Human Interferon-Alpha 14 Gene 284

L.1 Nucleotide Sequence 284

L.2 Restriction Endonuclease Sites 285

M Human Interferon-Alpha 16 Gene 296

M.1 Nucleotide Sequence 296

M.2 Restriction Endonuclease Sites 297

N Human Interferon-Alpha 17 Gene 307

N.1 Nucleotide Sequence 307

N.2 Restriction Endonuclease Sites 308

Bibliography 


\section{List of Tables}

1.1.1. Genbank DNA sequence data.

1.4.1. Thesis contributions.

2.1.1. Nucleic acid nomenclature.

2.2.1. Genetic code.

2.3.1. Restriction endonuclease action.

2.5.1. Nomenclature of the human interferon genes.

3.2.1. Current list of motif classes.

3.3.1. Summary of single-letter code nucleotide designations. 58

5.1. Human genes analyzed by nucleotide motif search. 66

5.2. Restriction endonuclease and transcription factor patterns. 66

5.1.1. Human interfercit-alpha 1 restriction and transcription analysis data. $\quad 71$

5.1.2. Human interferon-alpha 1 transcription control elements. 71

5.1.3. Human interferon-alpha 1 restriction and traniscription analysis summary. 71

5.2.1. Human interferon-alpha 2 restriction and transcription analysis data. 72

5.2.2. Human interferon-alpha 2 transcription control elements. 72

5.2.3. Human interferon-aipha 2 restriction and transcription analysis summary. 72

5.3.1. Human interferon-alpha 4 restriction and transcription analysis data. $\quad 73$

5.3.2. Human interferon-alpha 4 transcription control 'lements. 73

5.3.3. Human interferon-alpha 4 restriction and transcription analysis summary. 73

5.4.1. Human interferon-alpha 5 restriction and transcription analysis data. $\quad 74$

5.4.2. Human interferon-alpha 5 transcription control elements. 74

5.4.3. Human interferon-alpha 5 restriction and transcription analysis summary. 74

5.5.1. Human interferon-alpha 6 restriction and transcription analysis data. $\quad 75$ 
5.5.2. Human interferon-alpha 6 transcription control elements.

5.5.3. Human interferon-alpha 6 restriction and transcription analysis summary. $\quad 75$

5.6.1. Human interferon-alpha 7 restriction and transcription analysis data. $\quad 76$

5.6.2. Human interferon-alpha 7 transcription control elements. 76

5.6.3. Human interferon-alpha 7 restriction and transcription analysis summary. 76

5.7.1. Human interferon-alpha 8 restriction analysis data. 77

5.7.2. Human interferon-alpha 8 restriction and transcription analysis sumımary. 77

5.8.1. Human interferon-alpha 13 restriction and transcription analysis data. $\quad 78$

5.8.2. Human interferon-alpha 13 transcription contrcl elements.

5.8.3. Human interferon-alpha 13 restriction and transcription analysis summary. 78

5.9.1. Human interferon-alpha 14 restriction and transcription analysis data. $\quad 79$

5.9.2. Human interferon-alpha 14 transcription control elements. 79

5.9.3. Human interferon-alpha 14 restriction and transcription analysis summary. 79

5.10.1. Human interferon-alpha 16 restriction and transcription analysis data. $\quad 80$

5.10.2. Human interioron-alpha 16 transcription control elements. 80

5.10.3. Human interferon-alpha 16 restriction and transcription analysis summary. 80

5.11.1. Human interferon-alpha 17 restriction and transcription analysis data. $\quad 81$

5.11.2. Human interferon-alpha 17 transcription control elements. 81

5.11.3. Human interferon-alpha 17 restriction and transcription analysis summary. 81

5.12.1. Human retinoblastoma restriction and transcription analysis summary. $\quad 82$

$\begin{array}{ll}\text { 6.3.1. Promoter element consensus sequences. } & 105\end{array}$

6.4.1. Human interferon-alpha transcription regulatory sequences. 107

7.1.1. Parallel performance analysis experiments. 111

$\begin{array}{ll}\text { 7.1.2. Parallel performance analysis graphs. } & 111\end{array}$

7.2.1. Parallel perforn:aisce analysis of restriction endonuclease patterns. 112 
7.3.1. Parallel performance analysis of transcription factor patterns.

7.4.1. Parallel performance analysis of human interferon transcription factor patterns. 


\section{List of Figures}

1.1.1. Genbank DNA sequence growth curve (December, 1982 - August, 1994). 3

2.1.1. Nitrogenous bases and ribose sugars.

2.1.2. Deoxyadenosine monophosphate conformational model, generic nucleotide structure and deoxynucleotide structural formulas.

2.1.3. Polydeoxynucleotide segment.

2.1.4. DNA double helix unrolled to illustrate the sugar-phosphate backbones and base-pair rungs.

2.1.5. DNA duplex demonstrating the antiparallel polarity of the complementary strands.

2.1.6. Double-helical model of DNA.

2.1.7. B-DNA helix of the dodecamer CGCGAATTCGCG.

2.2.1. Generic amino acid structural formula, $R$ is an amino acid-specific side group.

2.2.2. Common amino acids found in proteins.

2.2.3. Portion of a protein molecule.

2.2.4. Tripeptide composed of three amino acids: alanine, valine and aspartic acid. 28

2.2.5. Human interferon-alpha 2 protein, backbone trace diagram.

2.5.1. Human chromosome 9 cytogenetic map featuring type-I interferon gene cluster locus.

2.5.2. Human type-I interferon gene cluster.

4.1.1. One-, two-, three- and four-dimensional hypercubes.

6.1.1. Human interferon alpha-1 genetic map. 
6.i.3. Human interferon alpha-4 genetic map. 86

6.1.4. Human interferon alpha-5 genetic map. 87

6.1.5. Human interferon alpha-6 genetic map. 88

6.1.6. Human interferon alpha-7 genetic map. $\quad 89$

6.1.7. Human interferon alpha-8 genetic map. 90

6.1.8. Human interferon alpha-13 genetic map. 91

6.1.9. Hunian interferon alpha-14 genetic map. 92

6.1.10. Human interferon alpha-16 genetic map. 93

6.1.11. Human interferon alpha-17 genetic map. 94

6.2.1. Human interferon-alpha 1 promoter transcription map. 95

6.2.2. Human interferon-alpha 2 promoter transcription map. 96

6.2.3. Human interferon-alpha 4 promoter transcription map. 96

6.2.4. Human interferon-alpha 5 promoter transcription map. 97

6.2.5. Human interferon-alpha 6 promoter transcription map. 97

6.2.6. Human interferon-alpha 7 promoter transcription map. 98

6.2.7. Human interferon-alpha 13 promoter transcription map. 98

6.2.8. Human interferon-alpha 14 promoter transcription map. 99

6.2.9. Human interferon-alpha 16 promoter transcription map. 99

6.2.10. Human interferon-alpha 17 promoter transcription map. 100

$\begin{array}{ll}\text { 6.3.1. Tata boxes. } & 101\end{array}$

$\begin{array}{ll}\text { 6.3.2. Virus-response elements. } & 102\end{array}$

6.3.3. Distal interferon regulatory factor sites. 104

6.3.4. Proximal interferon regulatory factor sites. 104

$\begin{array}{ll}\text { 6.3.5. TG sequences. } & 105\end{array}$ 
6.4.1. Human interferon-alpha transcription regulatory consensus sequence organization.

$\begin{array}{ll}\text { 7.2.1. Speedup curve of restriction endonuclease patterns. } & 113\end{array}$

7.2.2. Cost curve of restriction endonuclease patterns. $\quad 113$

$\begin{array}{ll}\text { 7.2.3. Efficiency curve of restriction endonuclease patterns. } & 114\end{array}$

7.3.1. Speedup curve of transcription factor patterns. 116

7.3.2. Cost curve of transcription factor patterns. 116

$\begin{array}{ll}\text { 7.3.3. Efficiency curve of transcription factor patterns. } & 117\end{array}$

7.4.1. Speedup curve of human interferon transcription factor patterns. $\quad 119$

7.4.2. Cost curve of human interferon transcription factor patterns. 119

$\begin{array}{ll}\text { 7.4.3. Efficiency curve of human interferon transcription factor patterns. } & 120\end{array}$ 


\section{Chapter 1}

\section{Introduction}

Open research problems in computational molecular biology are described. Precedent research in nucleotide motif analysis is reported. The nucleotide motif search problem is formally defined. Research findings and thesis organization are summarized.

\subsection{Genome Informatics}

The Human Genome Project is a concerted effort to systemically delineate genetic and physical maps of the total DNA complement of Homo sapiens, ultimately yielding the total nucleotide sequence of this DNA. The Human Genome Project has five objectives: 1. construction of a high resolution genetic map of the human genome, 2. production of a variety of physical maps of all human chromosomes and the chromosomes of selected model organisms, 3. determination of the complete sequence of human DNA and of the DNA of selected model organisms, 4. development of informatics capabilities to collect, store, analyze and distribute the data produced and 5. creation of the DNA sequencing and computing technologies necessary to achieve these objectives (Pearson and Soll, 1991).

Genome projects will generate enormuus amounts of information. Genbank 84.0, the August 1994 version of the U.S. national depository of nucleic acid sequences contains approximately 201 million nucleotides (Genbank, 1994). A major objective of the Human Genome Project is the development of more advanced DNA sequencing technologies. Concerted genome sequencing using these advanced technologies will result in further increases in the rate at which DNA sequences are obtained. DNA sequence growth rates are already creating significant bottlenecks in user accessibility and usage. The Genbank DNA sequence data exponential growth rate is presented (Table 1.1.1.) (Figure 1.1.1.). 


$\begin{array}{ccrr}\text { Release } & \text { Date } & \text { Nucleotides } & \text { Entries } \\ 3 & \text { Dec 82 } & 680338 & \\ 14 & \text { Nov 83 } & 2274029 & 2427 \\ 20 & \text { May } 84 & 3002088 & 3665 \\ 24 & \text { Sep 84 } & 3323270 & 4135 \\ 25 & \text { Oct 84 } & 3368765 & 4175 \\ 26 & \text { Nov 84 } & 3689752 & 4393 \\ 32 & \text { May 85 } & 4211931 & 4954 \\ 36 & \text { Sep 85 } & 5204420 & 5700 \\ 40 & \text { Feb 86 } & 5925429 & 6642 \\ 42 & \text { May 86 } & 6765476 & 7416 \\ 44 & \text { Aug 86 } & 8442357 & 8823 \\ 46 & \text { Nov 86 } & 9615371 & 9978 \\ 46 & \text { Feb 87 } & 10961380 & 10913 \\ 50 & \text { May 87 } & 13048473 & 12534 \\ 52 & \text { Aug 87 } & 14855145 & 14020 \\ 53 & \text { Sep 87 } & 15514776 & 14584 \\ 54 & \text { Dec 87 } & 16752872 & 15465 \\ 55 & \text { Mar 88 } & 19156002 & 17047 \\ 56 & \text { Jun 88 } & 20795279 & 18226 \\ 57 & \text { Sep 88 } & 22019698 & 19044 \\ 57.1 & \text { Oct 88 } & 23800000 & 20579 \\ 58 & \text { Dec 88 } & 24690876 & 21248 \\ 59 & \text { Mar 89 } & 26382491 & 22479 \\ 60 & \text { Jun 89 } & 31808784 & 26317 \\ 61 & \text { Sep 89 } & 34762585 & 28791 \\ 62 & \text { Dec 89 } & 37183950 & 31229 \\ 63 & \text { Mar 90 } & 40127752 & 33377 \\ 64 & \text { Jun 90 } & 42495893 & 35100 \\ 65 & \text { Sep 90 } & 49179285 & 39533 \\ 66 & \text { Dec 90 } & 51306092 & 41057 \\ 67 & \text { Mar 91 } & 55169276 & 43903 \\ 68 & \text { Jun 91 } & 65868799 & 51418 \\ 69 & \text { Sep 91 } & 71947426 & 55627 \\ 70 & \text { Dec 91 } & 77337678 & 58952 \\ 71 & \text { Mar 92 } & 83894652 & 65100 \\ 72 & \text { Jun 92 } & 92160761 & 71280 \\ 73 & \text { Sep 92 } & 101008486 & 78608 \\ 74 & \text { Dec 92 } & 120242234 & 97084 \\ 75 & \text { Feb 93 } & 126212259 & 106684 \\ 76 & \text { Apr 93 } & 129968355 & 111911 \\ 77 & \text { Jun 93 } & 138904393 & 120134 \\ 78 & \text { Aug 93 } & 147215633 & 131328 \\ 79 & \text { Oct 93 } & 157152442 & 143492 \\ 80 & \text { Dec 93 } & 163802597 & 150744 \\ 81 & \text { Feb 94 } & 173261500 & 162946 \\ 82 & \text { Apr 94 } & 180589455 & 169396 \\ 83 & \text { Jun 94 } & 191393939 & 182753 \\ 84 & \text { Aug 94 } & 201815802 & 196703\end{array}$

Table 1.1.1. Genbank DNA sequence data (Genbank, 1994). 


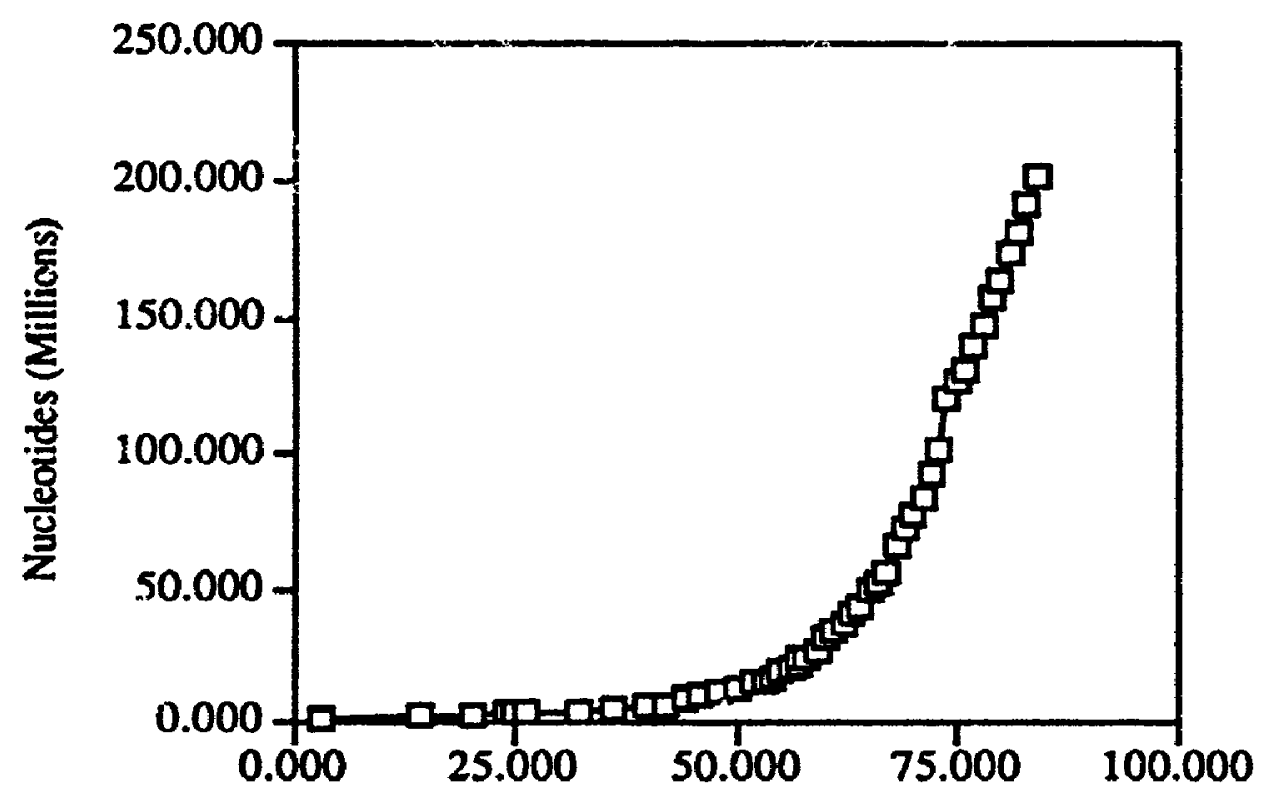

Release Number

Figure 1.1.1. Genbank DNA sequence growth curve (December, 1982 - August, 1994).

Computational biology refers to the study of biological phenomena through computational experiments. Computational molecular biology focuses on the computational interpretation of biological phenomena that involve nucleic acid and protein sequences. Genome informatics refers to the çomputational molecular biology aspects of genome projects. Genome informatics has identified five theoretical and computational problems amenable to solution by parallel computing: 1. database representations of genetic and physical maps, 2. combinatorial and statistical problems in map and sequence assembly, 3. prediction of protein structures from amino acid sequences, 4 . sequence similarity comparisons and 5. recognition of functionally-significant patterns in sequences.

1. Database representations of genetic and physical maps. Chromosome genetic linkage maps represent distances between markers based on meiotic recombination frequencies. Chromosome physical maps represent distances between rarkers based on 
numbers of nucleotides. Genomic databases should define four data types: 1 . genetic, 2. physical, 3. sequence and 4. bibliographic. Genetic data should include: 1. locus name, 2. location, 3. recombination distance, 4. polymorphisms, 5. breakpoints, 6. rearrangements and 7. disease association. Physical data should include: 1. restriction sites, 2. probe locations and 3. contigs. Sequence data should include: 1. sequence-tagged sites, 2. noncoding regions, 3. coding regions, 4. control regions, 5. telomeres, 6. centromeres, 7. repeats and 8. metaphase chromosome bands. Bibliographic references should cite primary scientific and medical literature.

Genomic databases are classified into four categories based on their contents: 1. bibliographic, 2. molecular, 3. genetic and 4. organism. Bibliographic databases include: 1. Medline, 2. Biological Abstracts (Biosis), 3. Chemical Abstracts Service (CAS) and 4. Agricola. Molectlar databases include: 1. European Molecular Biology Laboratory Nucleotide Sequence Data Library (EMBL), 2. Genbank, 3. Geninfo, 4. DNA Database of Japan (DDBJ), 5. Protein Identification Resource (PIR), 6. GenPept, 7. Swissprot, 8. Carbank, 9. BioMagRes (BMR) and 10. Protein Data Base (PDB). Genetic databases include: 1. Genome Data Base (GDB), 2. Online Mendelian Inheritance in Man (OMIM), 3. DRHPL and 4. Gbase. Organism databases include: 1. E. coli Genetic Stock Center and 2. American Type Culture Collection. Genomic databases have been reviewed (Pearson and Soll, 1991).

2. Combinatorial and statistical problems in map and sequence assembly. Genetic maps are cunstructed by assembling random DNA fragments into composite DNA sequences. The assembly procedure seeks to maximize the probable order and spacing of markers within a map.

The construction of a physical map typically requires the overlap and ordering of 1000 to 10000 partially-characterized clones to be established. First, the pairwise probability of clune overlap is estimated. Second, for those clones deduced to overlap with high probability a greedy algorithm is used to assemble the overlapping clones into contigs. 
During the process of contig construction, the characterization of a new clone may confirm or contradict an assigned overlap. Several weak though consistent overlaps may be necessary to acquire confidence in a contig. Therefore, a more systematic method for treating multiple overlapping clones for the entire contig would be preferable to simple pairwise overlaps. Interval graph theory may be useful in developing such an algorithm (Bell, 1990).

3. Prediction of protein structures from amino acid sequences. Protein folding refers to the mechanism by which the amino acid sequence of a protein adopts its functional three-dimensional conformation. The protein folding problem is the determination of the set of rules for predicting the native three-dimensional structure of a protein based entirely upon its amino acid sequence. The protein folding problem has been made more urgent by advances in DNA sequencing technology which have generated DNA sequences at a greater rate than the ability to isolate and characterize the protein products. The development of a folding code to complement the genetic code would complete the information transfer in the central dogma of molecular biology: DNA $\rightarrow$ RNA $\rightarrow$ protein $\rightarrow$ functional conformation.

Three methods have been developed to predict protein structure: 1 . substructure condensation, 2. homology modeling and 3. energetic methods. In substructure condensation, secondiry structures are predicted and these secondary structure units are then packed together to construct an approximate tertiary structure. Homology modeling exploits a recognizable sequence relationship between the protein under question and another protein of known structure to model a tertiary siructure. Energetic methods construct a potential energy function and follow the thermodynamic gradient to the optimal structure (energy minimization) or simulate the time-dependent motions of the polypeptide during the conformational transitions to a more thermodynamically-stable state (molecular dynamics). None of these prediction methods have been entirely successful (Ponder and Richards, 1987). Thus, the protein folding problem remains an open research question in 
structural molecular biology. The protein folding problem has been reviewed (Murphy and Freire, 1992) (Matthews, 1993).

4. Sequence similarity comparisons. Nucleic acid and protein sequence comparison is an important tool in genome informatics. Initial clues to understanding the structure or function of a macromolecular sequence arise from homologies to other macromolecules that have been previously studied. Genomic database searenes reveal biologically-significant sequence relationships and suggest future investigation strategies.

Molecular sequence database homology searching is affected by five factors: 1 . algorithms, 2. scoring systems, 3. alignment statistics, 4. database updates and 5. database sequence bias. 1. Algorithms. Database search algorithms are based upon measures of local sequence similarity. Algorithms must balance the competing factors of speed. hardware requirements and sensitivity to biological relationships. 2. Scoring systems. Alignments are ranked by ssores whose calculations are dependent upon the particular scoring systems used. The appropriate scoring system to use is largely dependant upon the problem under consideration. 3. Alignment statistics. Given a specific query, database search algorithms produce an ordered list of imperfectly-matched dataiase similarities. An important question is defining the critical point of statistical significance. 4. Database updates. The use of an up-to-date sequence database is essential to any similarity search. 5. Database sequence bias. There are biases in the molecules chosen to be included in nucleic acid and protein sequence databases. Issues in searching molecular sequence databases have been reviewed (Altschul et. al., 1994).

5. Recognition of functionally-significant patterns in sequences. Functional sites in DNA have been studied and partially characterized. DNA functional sites are sequences recognized and bound to by specific proteins, e.g. promster elements. DNA recognition sequences are difficult to characterize computationally. Recognition sequence algorithms exhibit performance tradeoffs between increasing sensitivity (ability to detect true positives) and decreasing selectivity (ability to exclude false positives). 
The identification of intron-exon boundaries and splice sites where RNA is transcribed from genomic DNA is of further importance. The ability to accurately predict introns would greatly facilitate the translation of genomic DNA into the amino acid sequence of the gene product.

Comparative analysis of DNA sequences is an important technique in d-tecting biologically-significant relationships. Multiple sequence alignment is a useful technique in analyzing sequence-structure relationships. The DNA sequence of an unknown gene often exhibits structural homology with a known gene. Thus, homology relatiorships frequently permit biological and functional insights to be made regarding the mechanism of action of the unknown gene. Multiple sequence alignment is important for the recognition of patterns or motifs common to a set of functionally-related DNA sequences and is of assistance in structure prediction and molecular modeling. Multiple sequence alignment algorithms use variations of the dynamic programming method. Dynamic programming methods use an explicit measure of alignment quality, consisting of defined costs for aligned pairs of residues or residues with gaps and use an algorithm for finding an alignment with minimum total cost. Multiple sequence alignment algorithms have been reviewed (Chan et. al., 1992).

Genome analysis has been identified as a Grand Challenge problem in computational science. Genome informatics has been reviewed (Smith, 1994). 


\subsection{Nucleotide Motifs}

Nucleotide motifs are conserved patterns of nucleotides that are common to a group of functionally-related sequences and often encode a discrete function, e.g. the tata box is a nucleotide sequence required for accurate transcription initiation. Nucleotide motifs are frequently compiled into machine-readable collections for the purposes of database searching and automated motif detection (Ghosh, 1993). Motifs are usually derived from the multiple alignment of a group of related sequences. Motifs are usually presented as consensus sequences. Cunsensus sequences are useful for computational searches along DNA sequences.

Consider the nucleotide motif search problem. The nucleotide motif search input consists of two items: 1. a finite set of nucleotide motifs and 2. a DNA sequence. The sum of the lengths of the nucleotide motifs is defined as $m$. The length of the DNA sequence is defined as $\mathrm{n}$. Precendent solutions have been proposed for the nucleotide motif search problem. The GCG Map program finds restriction endonuclease recognition sites using a brute force algorithm in $O(\mathrm{~nm})$ time (Devereux et. al., 1984). The Signal Scan 3.0 program determines transcription factor binding sites using an indexed brute force algorithm of undetermined time complexity (Prestridge and Stormo, 1993). Restriction endonuclease recognition sites are identified using a nondeterministic finite automaton algorithm of undetermined time complexity (Mount and Conrad, 1986). Nondeterministic finite automaton algorithms are superior to brute force algorithms but have the disadvantage of a stack requirement for execution. The stack requirement can be eliminated using a deterministic finite automaton. Restriction endonuclease recognition sites are identified using a deterministic finite automaton algorithm in $O(n+m)$ time (Smith, 1988). The Intelligenetics PC Gene Restri program finds restriction endonuclease recognition sites using an unidentified algorithm. 
Motifs have varying specificity. Because of the problem of false positives, the discovery of a particular motif in a sequence should be interpreted cautiously. Motifs are nevertheless useful for making functional inferences concerning a novel DNA sequence (Boguski, 1992). 


\subsection{Notation and Problem Statement}

Numerous sequence analysis problems in computational molecular biology can be expressed as nucleotide motif search problems, e.g. restriction analysis and transcription analysis. Nucleotide motif search is a specialized instance of the multiple keyword search problem. A multiple keyword search algorithm was developed for searching for class 1 nucleotide motifs within DNA sequences.

Consider the keyword $\mathbf{k}$ and the text $\mathbf{t}$ to be two strings defined over an arbitrary alphabet $\Sigma$. The keyword length is defined as $m_{1}|k|=m$. The text length is defined as $n$, $|t|=n$. The keyword length $m$ is typically much shorter than the text length $n, m \leq n$. The keyword search problem is to find all occurrences of the keyword as a substring within the text. The output is the set of positions in the text where the keyword occurs.

The multiple keyword search problem is a generalization of the keyword search problem. Consider a finite set of $y$ keywords $k_{1}, k_{2}, \ldots k_{y}$ and a text $t$ defined as strings over an arbitrary alphabet $\Sigma$. The multiple keyword search problem is to find all occurrences of each keyword as a substring within the text. The output is the set of positions in the text where any of the keywords occur.

A motif is defined as a continuous segment of a macromolecular sequence. The class 1 rucleotide motif involves an exact match to a short defined sequence (Staden, 1990). Class 1 nucleotide motifs occur within DNA as transcription factor binding sites and restriction endonuclease recognition sites.

The nucleotide motif search problem may be stated as follows. Consider a finite set of $y$ class 1 nucleotide motifs $k_{1}, k_{2}, \ldots k_{y}$ and a DNA sequence $t$ defined as sequences over the alphabet $\Sigma_{D N A}=\{A, C, G, T\}$. The nucleotide motif search problem is to find all occurrences of each nucleotide motif as a subsequence within the DNA sequence. The output is the set of positions in the DNA where any of the nucleotide motifs occur. 
In DNA sequence analysis, nucleotide motif search is a specialized instance of the multiple keyword search problem. Two DNA sequence analysis problems can be expressed as nucleotide motif search problems: 1 . restriction analysis and 2. transcription analysis. Thus, the identification of transcription factor binding sites and restriction endonuclease recognition sites within an arbitrary DNA sequence are specialized cases of the multiple keyword search problem. 


\subsection{Thesis Contributions}

A multiple keyword search algorithm was developed for the Intel iPSC/860 Hypercube. The algorithm is specialized to searching for class 1 nucleotide motifs within DNA sequences (Staden, 1990). Consider a hypercube composed of p processors, a DNA sequence of length $\mathrm{n}$ nucleotides and a list of nucleotide motifs of total $\mathrm{m}$ nucleotides. Under such conditions, the parallel class 1 nucleotide motif search algorithm would require $O(m+(n / p))$ time and occupy $O(n+m p)$ space.

The nucleotide motif search algorithm was used to characterize the human interferon-alpha multigene family. Eleven human interferon-alpha genes were subjected to restriction analysis and transcription analysis: IFNA1, IFNA2, IFNA4, IFNA5, IFNA6, INFA7, IFNA8, IFNA13, IFNA14, INFA16 and INFA17. Nucleotide motif analysis of the human interferon-alpha genes yielded six types of genetic markers: 1. restriction endonuclease sites, 2. virus response elements, 3. distal interferon regulatory factor sites, 4. proximal interferon regulatory factor sites, 5. TG sequences and 6. polyadenylation signals. The nucleotide motif sites were visualized to pruduce human interferon-alpha restriction maps and transcription maps. On the basis of computational evidence, a theoretical model for the transcriptional control of human interferon-alpha gene expression was proposed. The distribution of original research findings among the human interferonalpha genes is summarized (Table 1.4.1.). 
Data

nucleotide motif analysis algorithm restriction endonuclease sites restriction maps virus response elements distal interferon regulatory factor sites proximal interferon regulatory factor sites TG sequences polyadenylation signals transcription maps transcription regulatory mechanism
Human Interferon-Alpha Genes

$1,2,4,5,6,7,8,13,14,16,17$

$1,2,4,5,6,7,8,13,14,16,17$

$1,2,4,5,6,7,8,13,14,16,17$

$6,16,17$

$2,4,5,6,7,13,14,16,17$

$2,4,5,6,7,13,14,16,17$

$2,4,5,6,7,13,14,16,17$

$1,2,17$

$1,2,4,5,6,7,13,14,16,17$

$1,2,4,5,6,7,13,14,16,17$

Table 1.4.1. Thesis contributions. 


\subsection{Thesis Outline}

The remainder of the thesis is organized as follows. Chapter 2 describes seven topics in molecular biology: 1. nucleic acids, 2. protein, 3. restriction endonucleases, 4. transcription factors, 5. interferon, 6. retinoblastoma and 7. Human Genome Project. Chapter 3 defines the nucleotide motif search algorithm. Chapter 4 defines the nucleotide motif search algorithm implementation on the Intel iPSC/860 Hypercube. Chapter 5 presents the human interferon-alpha nucleotide motif search results for restriction endonuclease recognition sites and transcription factor binding sites. Chapter 6 presents a genetic analysis of the human interferon-alpha multigene family. Chapter 7 presents a performance analysis of the parallel nucleotide motif search algorithm using restriction endonucleases and transcription factors. Chapter 8 concludes with a summary of the nucleotide motif search algorithm and the human interferon-alpha genetic analysis. Future directions for parallel nucleotide motif search algorithm research and human interferonalpha transcriptional regulation research are proposed. 


\section{Chapter 2}

\section{Molecular Biology}

Molecular biology is a modern branch of science concerned with the study of biological phenomena at the molecular level. Molecular genetics is a subject focusing on the properties of deoxyribonucleic acid. Molecular genetics uses biochemical and biophysical techniques to investigate genetic problems. Seven topics in molecular biology are presented: 1. nucleic acids, 2. protein, 3. restriction endonucleases, 4. transcription factors, 5. interferon, 6. retinoblastoma and 7. Human Genome Project.

1. Nucleic acids. DNA and RNA are nucleic acids responsible for the storage and transmission of genetic information. Nucleic acid biochemistry is discussed.

2. Protein. Proteins are polypeptides responsible for cellular structure and metabolism. Protein biochemistry and the genetic code are discussed.

3. Restriction endonucleases. Type II restriction endonucleases recognize and cleave double-stranded DNA at specific sequences. Restriction endonucleases are used as analytical tools in recombinant DNA technology. Restriction maps of the human interferon-alpha multigene family were determined.

4. Transcription factors. Transcription factors are a class of DNA-binding proteins that bind to specific DNA sequences in gene regulatory regions and control gene expression. Transcription maps of the human interferon-alpha multigene family were determined.

5. Interferon. The interferons are a class of potent multifunctional cytokines that form a primary defense mechanism against viral infections and neoplasms. The human interferon-alpha multigene family was subjected to nucleotide motif analysis.

6. Retinoblastoma. The retinoblastoma gene is a tumor suppressor gene that negatively regulates cell proliferation. The retinoblastoma gene spans 180,388 nucleotides 
of genomic DNA and forms the longest known contiguous human DNA sequence. Thus, retinoblastoma DNA was used as the experimental subject in nucleotide motif search algorithm parallel performance experiments.

7. Human Genome Project. The Human Genome Project is an international research initiative with the goal of producing detailed genetic and physical maps of the 24 different human chromosomes. The significance of genetic mapping and sequencing is rationalized.

\subsection{Nucleic Acids}

Nucleic acids are macromolecules that function to store and transmit genetic information. Nucleic acids are polynucleotides, i.e. polymers composed of a sequence of nucleotide monomers. There are two kinds of nucleic acids: 1 . deoxyribonucleic acid (DNA) and 2. ribonucleic acid (RNA).

DNA stores genetic information. A DNA nucleotide sequence is converted to a corresponding RNA nucleotide sequence in a process ieferred to as transcription. The RNA nucleotide sequence is subsequently converted to a corresponding protein amino acid sequence in a process referred to as translation. The DNA sequence containing the genetic information required for the synthesis of a functional biological product (RNA or protein) is referred to as a gene. A cell typically contains thousands of genes. Thus, DNA molecules are very large.

RNA transmits genetic information from DNA to protein. There are three classes of RNA molecules: 1. ribosomal RNA, 2. messenger RNA and 3. transfer RNA. Ribosomal RNAs (rRNA) are structural components of ribosomes, the large complexes that synthesize proteins. Messenger RNAs (mRNA) act as intermediate molecules moving the information specifying a gene from the DNA of the nucleus to the ribosome, where the corresponding proteins are synthesized. Tronsfer RNAs (tRNA) are adapter molecules that transtate the 
genetic information in an mRNA molecule into the corresponding sequence of amino acids that comprise a protein.

A nucleotide has three components: 1. a nitrogenous base, 2. a pentose and 3. a phosphate. A nucleoside has two componerts: 1. a nitrogenous base and 2. a pentose. Thus, a nucleotide can be considered the 5 ' monophosphorylated derivative of a nucleoside. The nitrogenous bases and pentoses are heterocyclic compounds in which the carbon and nitrogen atoms are numbered.

1. Nitrogenous base. Nitrogenous bases are derivatives of two parent compounds: 1. purine and 2. pyrimidine. Nucleic acids possess two major purine derivatives: 1 . adenine (A) and 2. guanine (G). Adenine and guanine are found in both DNA and RNA. Nucleic acids possess three major pyrimidine derivatives: 1 . cytosine (C), 2. thymine (T) and 3. uracil $(\mathrm{U})$. Cytosine and thymine are found in DNA while cytosine and uracil are found in RNA. Thymine residues in DNA correspond to uracil residues in RNA. Nitrogenous base structural formulas are presented (Figure 2.1.1.).

2. Pentose. A pentose is a 5-carbon cyclic sugar. Nucleic acids possess two kinds of pentoses: 1. 2-deoxyribose and 2. ribose. 2-deoxyribose is found in DNA while ribose is found in RNA. The difference between a 2-deoxyribose and a ribose lies solely on the $2^{\prime}$ hydroxyl group on ribose. Thus, the major structural distinction between DNA and RNA is based on the sugar residues: DNA contains only 2-deoxyribose while RNA contains only ribose. Pentose structural formulas are presented (Figure 2.1.1.).

3. Phosphate. The pyrophosphate group $\mathrm{PO}_{4}$ is linked to the hyroxyl group on the pentose $5^{*}$ carbon.

Deoxyribonucleotides (deoxyribonucleoside $5^{\prime}$-monophosphates) are the structural units of DNAs while ribonucleotides (ribonucleoside 5'-monophosphates) are the structural unite of RNAs. The nucleotide sequence is termed the primary structure of a nucleic acid. The prinary structure stores the genetic information of a DNA molecule. Nucleotide 
structural formulas are presented (Figure 2.1.2.). Nucleic acid nomenclature is presented (Table 2.1.1.).<smiles>O=C1CCN[C@H](O)N1</smiles><smiles>CC1=CNC(O)NC1=O</smiles><smiles>OC[C@H]1O[C@H](O)[C@H](O)[C@H](O)[C@H]1O</smiles>

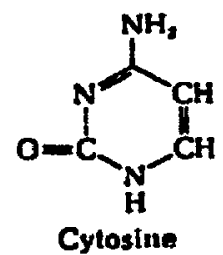

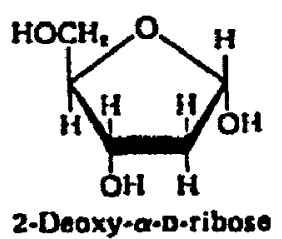<smiles>NC1NCNc2[nH]cnc21</smiles>

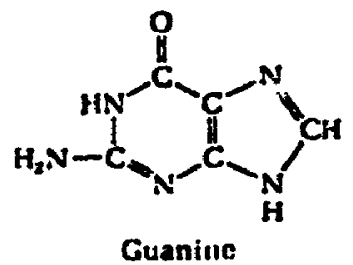

Figure 2.1.1. Nitrogenous bases and ribose sugars (Lehninger, 1982). 

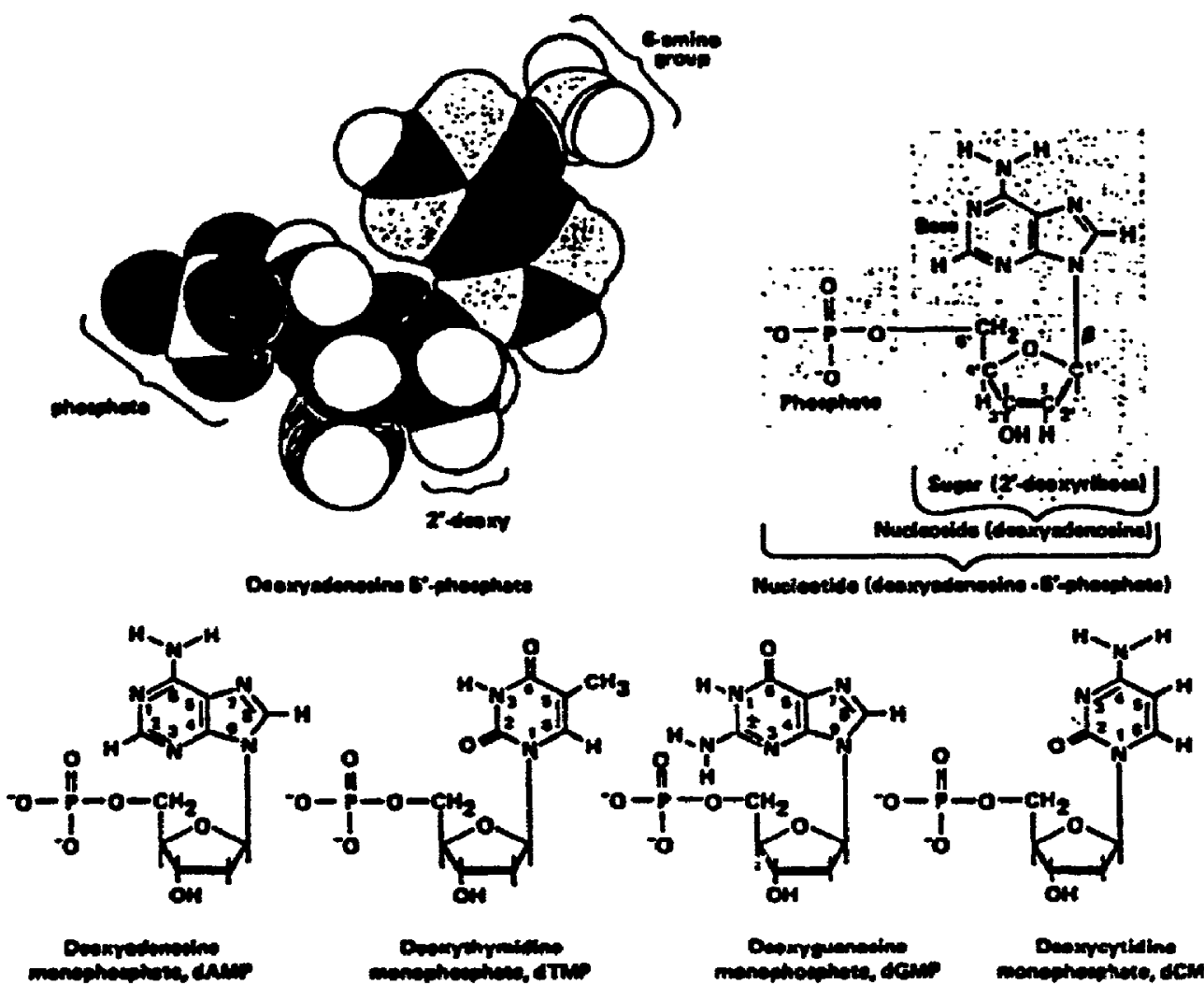

Denorym

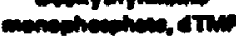

Figure 2.1.2. Deoxyadenosine monophosphate conformational model, generic nucleotide structure and deoxynucleotide structiral formulas (Komberg and Baker, 1992).

\begin{tabular}{|c|c|c|c|}
\hline Category & Base & Nucleoside & Nucleotide \\
\hline Purines & $\begin{array}{l}\text { adenine } \\
\text { guanine }\end{array}$ & $\begin{array}{l}\text { adrnosine } \\
\text { deoxyadenosine } \\
\text { guanosize } \\
\text { deoxyguanosine }\end{array}$ & $\begin{array}{l}\text { adenylate } \\
\text { deoxyadenylate } \\
\text { guanylate } \\
\text { deoxyguanylate }\end{array}$ \\
\hline Pyrimidines & $\begin{array}{l}\text { cytosine } \\
\text { thymine } \\
\text { uracil }\end{array}$ & $\begin{array}{l}\text { cytidine } \\
\text { deoxycytidine } \\
\text { thymidine } \\
\text { uridine }\end{array}$ & $\begin{array}{l}\text { cytidylate } \\
\text { deoxycytidylate } \\
\text { thymidylate } \\
\text { uridvlate }\end{array}$ \\
\hline
\end{tabular}

Table 2.1.1. Nucleic acid nomenclature (Komberg and Baker, 1992). 
Polynucleotide chains are long unbranched polymers formed by phosphodiester bonds between the 5'-hydroxyl group of one nucleotide and the 3'-hydroxyl group of the next nucleotide. Thus, the covalent backbones of nucleic acids consist of alternating phosphate and pentose residues. The nitrogenous bases are side groups that are joined to the sugar-phosphate backbone at regular intervals.

The nucleotide sequences of nucleic acids can be represented schematically. Nitrogenous bases are sumnarized by four letters: A, C, G and T, 2-deoxyriboses are represented as either vertical or horizontal lines. 2-deoxyribose carbon atoms are represented from $1^{\prime}$ at the top to $5^{\prime}$ at the bottom of the vertical line. Phosphodiester bonds are symbolized by the letter $P$ within a diagonal line. The connecting lines between nucleotides are drawn diagonally from the middle $\left(3^{\prime}\right)$ of the 2-deoxyribose of one nucleotide to the bottom (5') of the 2-deoxyribose of the next nucleotide.

DNA chains exhibit polarity. One end of the chain has a 5'-OH group and the other end has a $3^{\prime}-\mathrm{OH}$ group that is not linked to another nucleotide. By convention, an oligonucleotide is written with the unreacted 5'-OH group on the left end and the unreacted $3^{\prime}-\mathrm{OH}$ group on the right end. Thus, the nucleotide sequence is written in the $5^{\prime} \rightarrow 3^{\prime}$ direction.

Polynucleotides can be written in abbreviated formats. An oligonucleotide with an unreacted 3'-OH would be represented: pApCpGpT. An oligonucleotide with an unreacted 5'-OH would be represented: ApCpGpTp. The most compact nomenclature for the nucleotide sequence would be: ACGT. Polynucleotide structural and schematic formulas are presented (Figure 2.1.3.). 


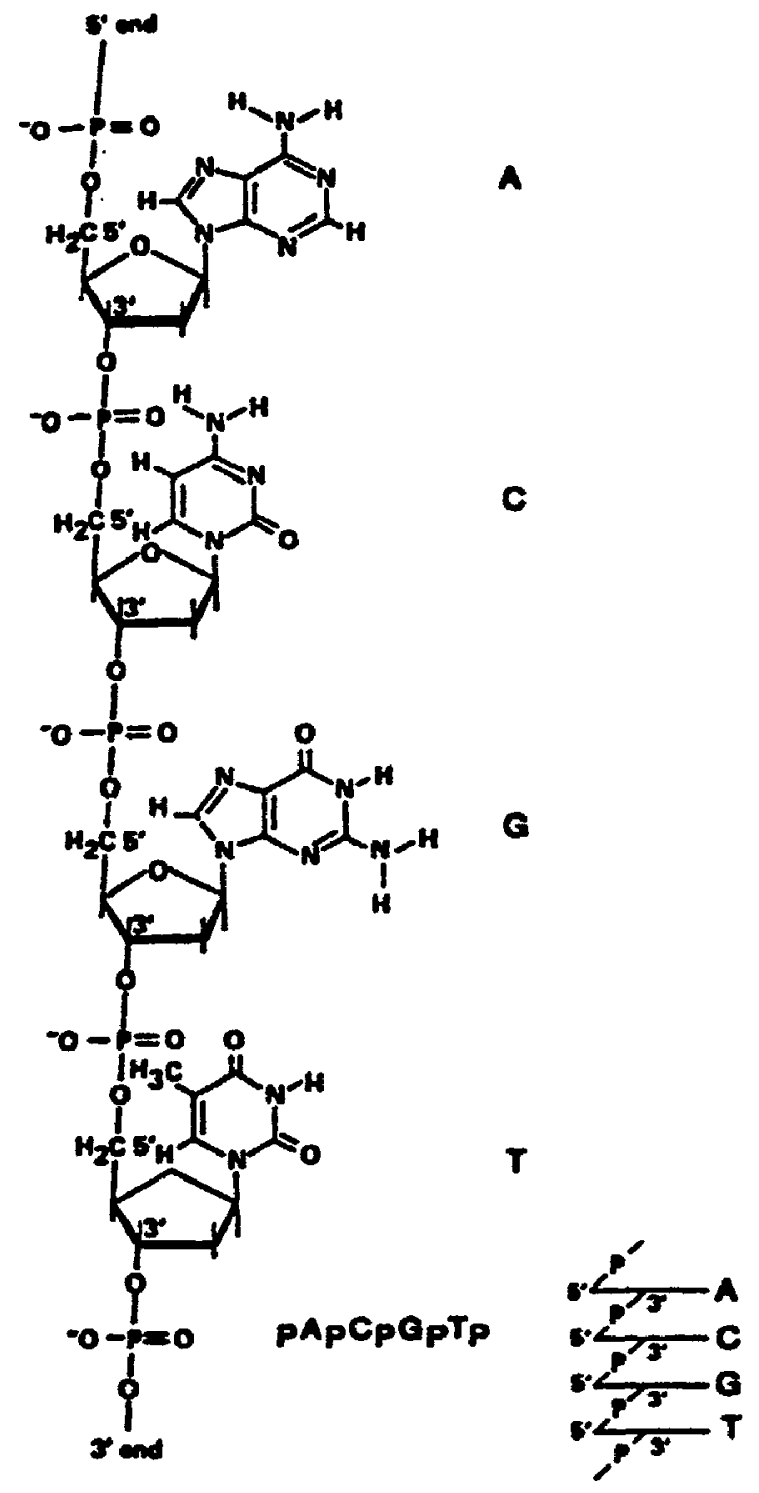

Figure 2.1.3. Polydeoxynucleotide segment (Komberg and Baker, 1992). 
Two polynucleotide strands align themselves to form a double helix. Nucleotides from opposite strands use hydrogen bonds to form complementary polynucleotide strands. Two interacting nucleotides from opposite polynucleotide strands are referred to as a base pair. There are two types of base pairs: 1. adenine-thymine and 2. cytosine-guanine. The adenine-thymine (AT) base pair forms two hydrogen bonds. The cytosine-guanine (CG) base pair forms three hydrogen bonds. The two types of base pairs occupy the same amount of space, permitting the DNA double helix to be of uniform diameter. Base-pair interaction between two complementary polynucleotide strands is presented (Figure 2.1.4.).

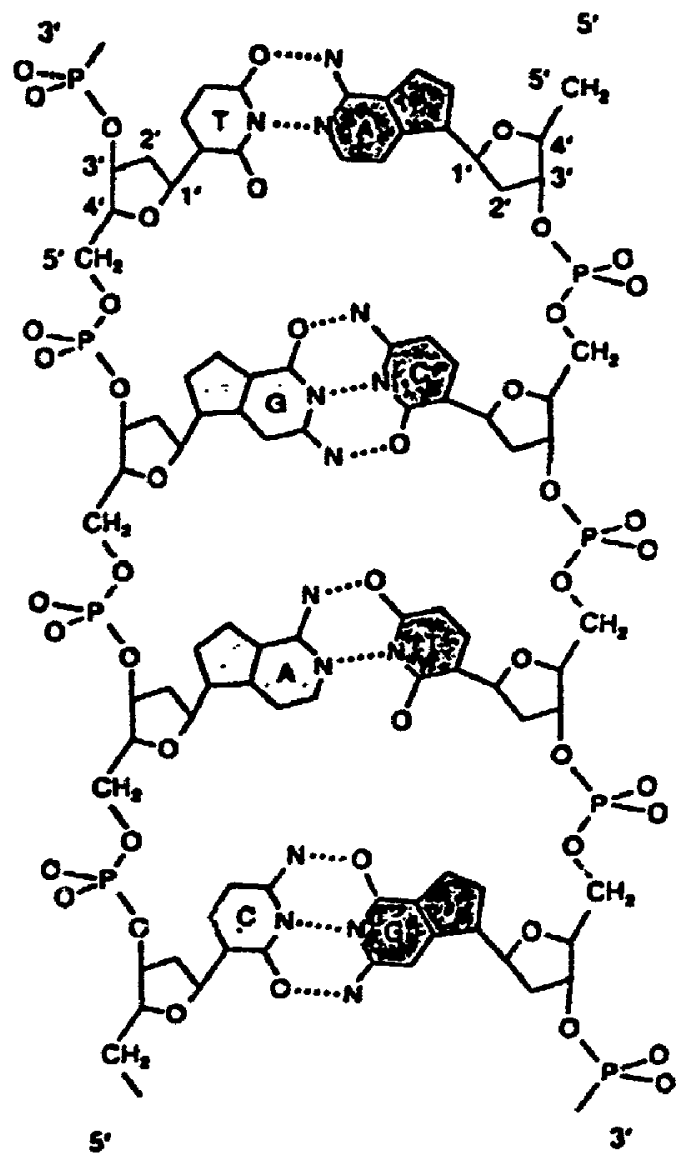

Figure 2.1.4. DNA double helix unrolled to illustrate the sugar-phosphate backbones and base-pair rungs (Juzuki et. al., 1989). 
The two polynucleotide chains of a DNA double helix are antiparallel. The $5^{\prime} \rightarrow 3^{\prime}$ phosphodiester bonds run in opposite directions. The antiparailel polarity of the two complementary polynuclectide strands is presented (Figure 2.1.5.).

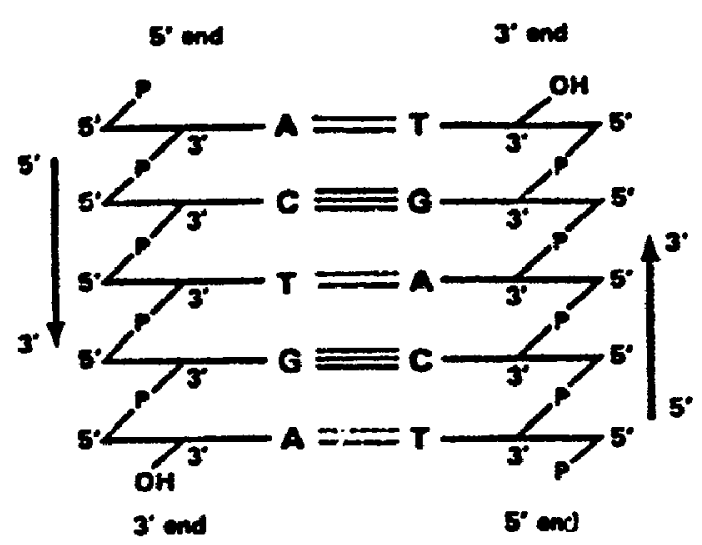

Figure 2.1.5. DNA duplex demonstrating the antiparallel polarity of the complementary strands (Komberg and Baker, 1992).

Base-pairing between nucleotides is responsible for the alignment of two polynucleotide strands to form a stabilized double helix. The base-pairing complementary of the two strands accounts for the accurate replication of DNA and the accurais transcription of RNA. The three-dimensional topology is referred to as the secondary structure of a nucleic acid.

DNA can occur in five conformations: A, B, C, D and Z. The B conformation is the most stable structural form under physiological conditions and is therefore the standard reference point for DNA architecture. R-conformation DNA helix parameters were elucidated using $\mathrm{x}$-ray crystallography.

The sugar-phosphate backbones of the two chains form a right-handed double helix with a $20 \mathrm{~A}$ diameter. The double helix has a wide major groove and a narrow minor groove. The sugar-phosphate backbones of the two chains are on the exterior of the molecule and are connected as a ladder, with the ladder rungs being the nitrogenous bases 
connected by hydrogen bonds. The nitrogenous base surface planes are perpendicular to the helical axis. Neighboring bases are spaced 3.4 $\mathrm{A}$ apart. There are ten base pairs per helix turn. Thus, each base pair is rotated $36^{\prime}$ relative to its neighbor. Each full turn has a length of $34 \mathrm{~A}$. The standard B-conformation double-helical model of DNA is presented (Figure 2.1.6.) (Figure 2.1.7.).

The three-dimensional structure of DNA has been reviewed (Zimmerman, 1982) (Dickerson, 1992).

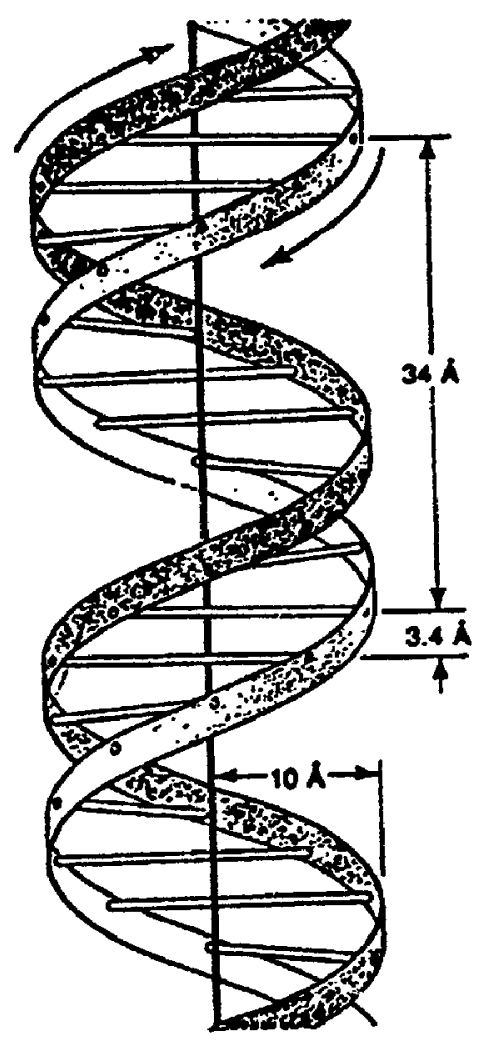

Figure 2.1.6. Double-helical model of DNA. Sticks represent base pairs and the ribbons represent the sugar-phosphate backbones of the two antiparallel stranas (Suzuki et. al., 1989). 
25

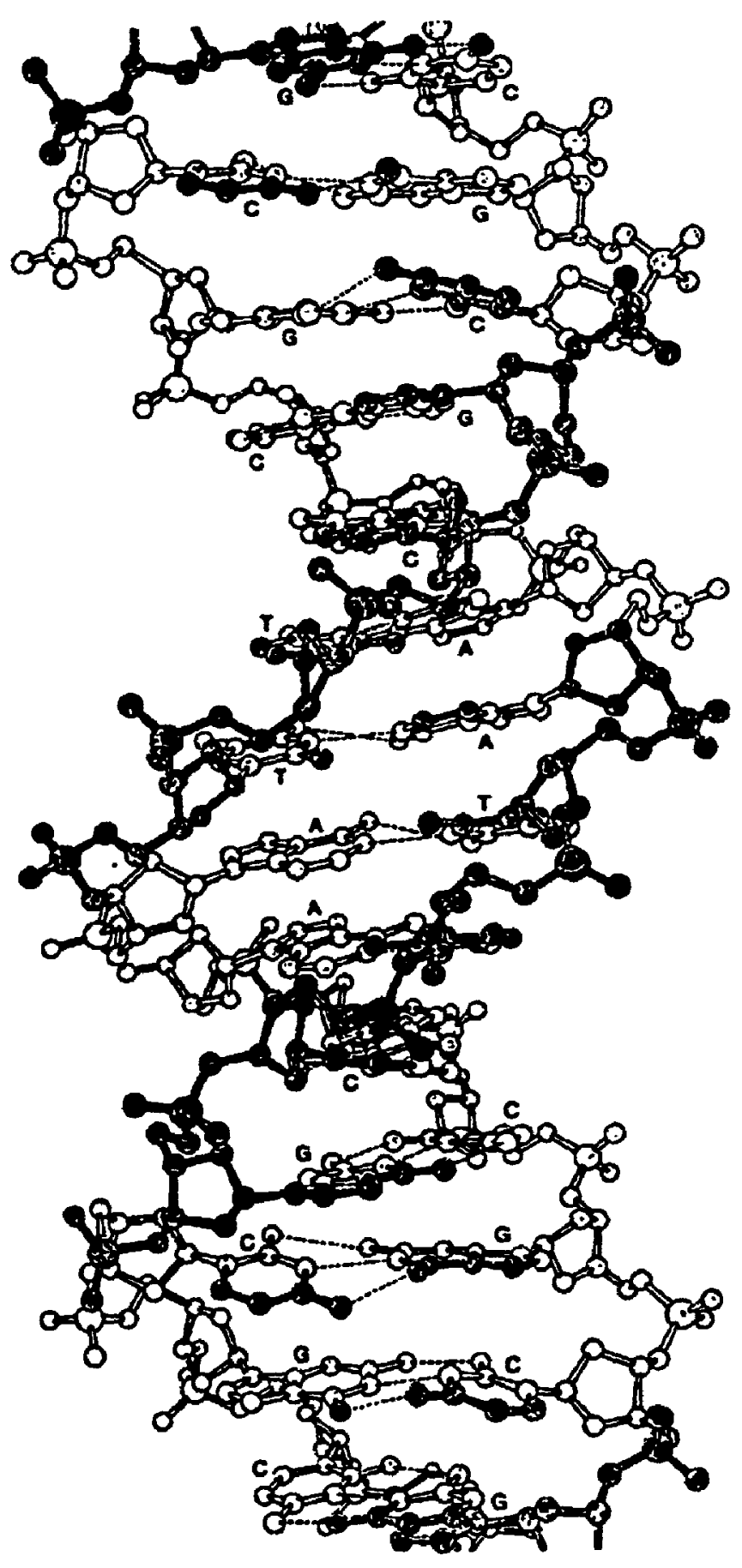

Figure 2.1.7. B-DNA helix of the dodecamer CGCGAATTCGCG (Suzuki et. al., 1989). 


\subsection{Protein}

Proteins are macromolecules that are responsible for cellular structure and metabolism. Proteins catalyze biochemical reactions which perform numerous functions, e.g. gene regulation. Proteins are polypeptides, i.e. polymers composed of a sequence of amino acid monomers.

The generic amino acid formula is $\mathrm{H}_{2} \mathrm{~N}-\mathrm{CHR}-\mathrm{COOH}$, where $\mathrm{R}$ is an amino acidspecific side group which varies from a hydrogen atom in the case of the amino acid glycine, to a complex ring in the case of the amino acid tryptophan. There are 20 common anino acids found in living organisms. The generic amino acid structural formula is presented (Figure 2.2.1.).<smiles>[R]C(N)C(=O)O</smiles>

Figure 2.2.1. Generic amino acid structural formula, $R$ is an ammo acid-specific side group.

The amino acid structural formulas are presented (Figure 2.2.2.).

Amino acids can be covalently linked through a substituted amide linkage termed a peptide bond to form a peptide. Such a linkage is formed by the removal of a water molecule (dehydration) from the carboxyl group of one amino acid and the amino group of the other amino acid. Thus, peptide-bond formation :: an example of a condensation reaction. A peptide bond is presented (Figure 2.2.3.). 

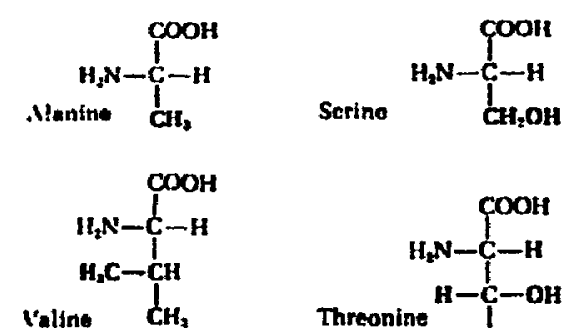<smiles>CC(C)CC(N)C(=O)O</smiles><smiles>CCC(C)C(N)(CC)C(=O)O</smiles><smiles>O=C(O)C1CC2CCC1N2</smiles>

Proline<smiles>CC(N)C(=O)O</smiles>

Gilycine

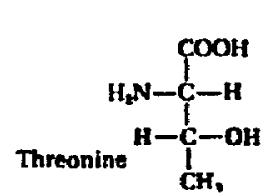

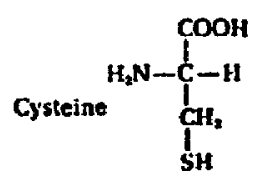<smiles>N[C@H](Cc1ccc(O)cc1)C(=O)O</smiles><smiles>CC(C(N)C(=O)O)C1CNc2ccccc21</smiles>
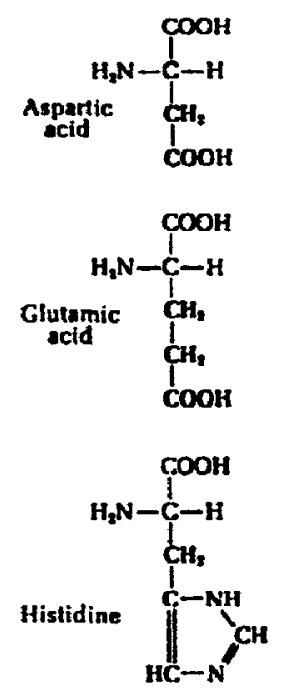<smiles>NC(O)CC(N)C(=O)O</smiles><smiles>NC(Cc1ccccc1)C(=O)O</smiles>

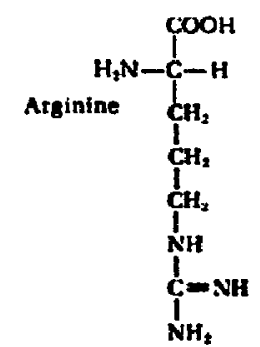<smiles>NCCCCC(N)C(=O)O</smiles><smiles>CSCCC(N)C(=O)O</smiles><smiles>N[C](N)CCCC(N)C(=O)O</smiles>

Figure 2."2.2. Common amino aciơs found in proteins (Lehninger, 1982).

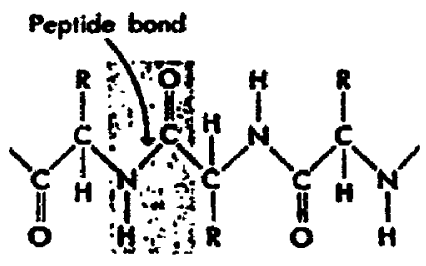

Figure 2.2.3. Portion of a protein molecule. Three amino acids aze linked together by peptide bonds (Watson et. al., 1987). 
Several amino acids linked together by peptide bonds form a molecule referred to as a polypeptide. The amino acid units in a peptide are often referred to as residues. The amino acid residue at the end of a peptide having a free amino group is referred to as the amino-terminal (N-terminal) residue. The residue at the opposite end of the peptide having a free carboxyl group is referred to as the carboxyl-terminal (C-terminal) residue. Oligopeptide names are derived from the sequence of their constituent amino acids, beginning with the amino terminus on the left and proceeding towards the carboxyl terminus on the right. The tripeptide composed from the three amino acids alanine, valine and aspartic acid is presented (Figure 2.2.4.).
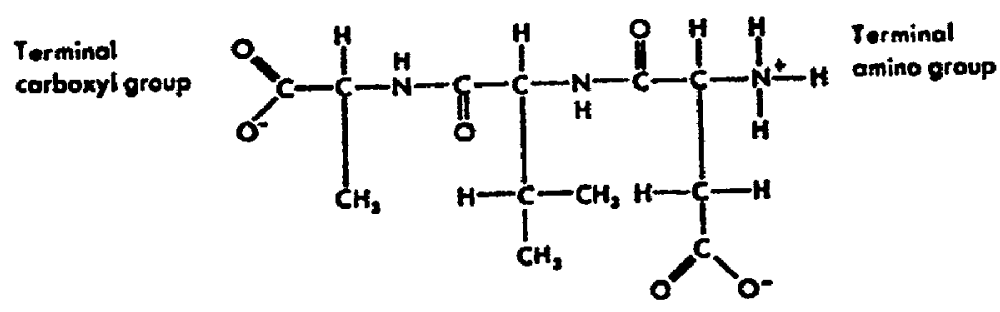

Figure 2.2.4. Tripeptide composed of three amino acids: alanine, valine and aspartic acid (Watson et. al., 1987).

The sequence of amino acids which constitutes a protein can be represented as a sequence of characters from a twenty-character alphabet. This structure is encoded in DNA by the genetic code in which each amino acid is represented by a triplet of DNA nucleotides. DNA is a sequence of nucleotides which can be represented as a sequence of characters from a four-character alphabet. Protein synthesis is called translation because the information contained in the four-character nucleotide alphabet is translated into the twenty-character amino acid alphabet.

The linear arrangement of amino acids in a polypeptide chain is referred to as the primary structure of the protein. The side groups of amino acids attract or repel one 
another resulting in different types of secondary structure of the protein. Two examples of secondary structures include: 1 . the $\alpha$-helix and 2. the $\beta$-pleated sheet. Proteins can be further folded to form a tertiary structure. Several folded structures can associate to form a quartemary structure, e.g. the oxygen-transporting protein hemoglobin is composed of four protein subunits.

Human interferon-alpha 2 (IFNA2) is a cytokine with broad-spectrum antiviral, antiproliferative and immunomodulatory properties. Human interferon-alpha 2 inhibits viral replication, inhibits malignant cell growth, regulates oncogene expression and activates natural killer cells. Human interferon alpha 2 is effective against eight human diseases: 1. hairy cell leukemia, 2. Kaposi's sarcoma, 3. chronic myelogenous leukemia, 4. non-Hodgkin's lymphoma, 5. chronic granulomatous disease, 6. condyloma acuminatum, 7. juvenile laryngeal papillomatosis and 8. chronic infection with hepatitis B and $C$ viruses (Sen and Lengyel, 1992).

Human interferon-alpha 2 codes for a protein having a signal peptide of 23 amino acids and a mature peptide of 165 amino acids with a calculated molecular weight of 19,390 daltons (Goeddel et. al., 1980) (Maeda et. al., 1980) (Streuli et. al., 1980) (Goeddel et. al., 1981) (Lawn et. al., 1981b) (Bowden et. al., 1984). The three-dimensional structure of the human interferon-alpha 2 protein has been recently determined based on a homology model with the murine interferon-beta protein (Murgolo et. al., 1993). The human interferon-alpha 2 protein backbone trace diagram is presented (Figure 2.2.5.). 


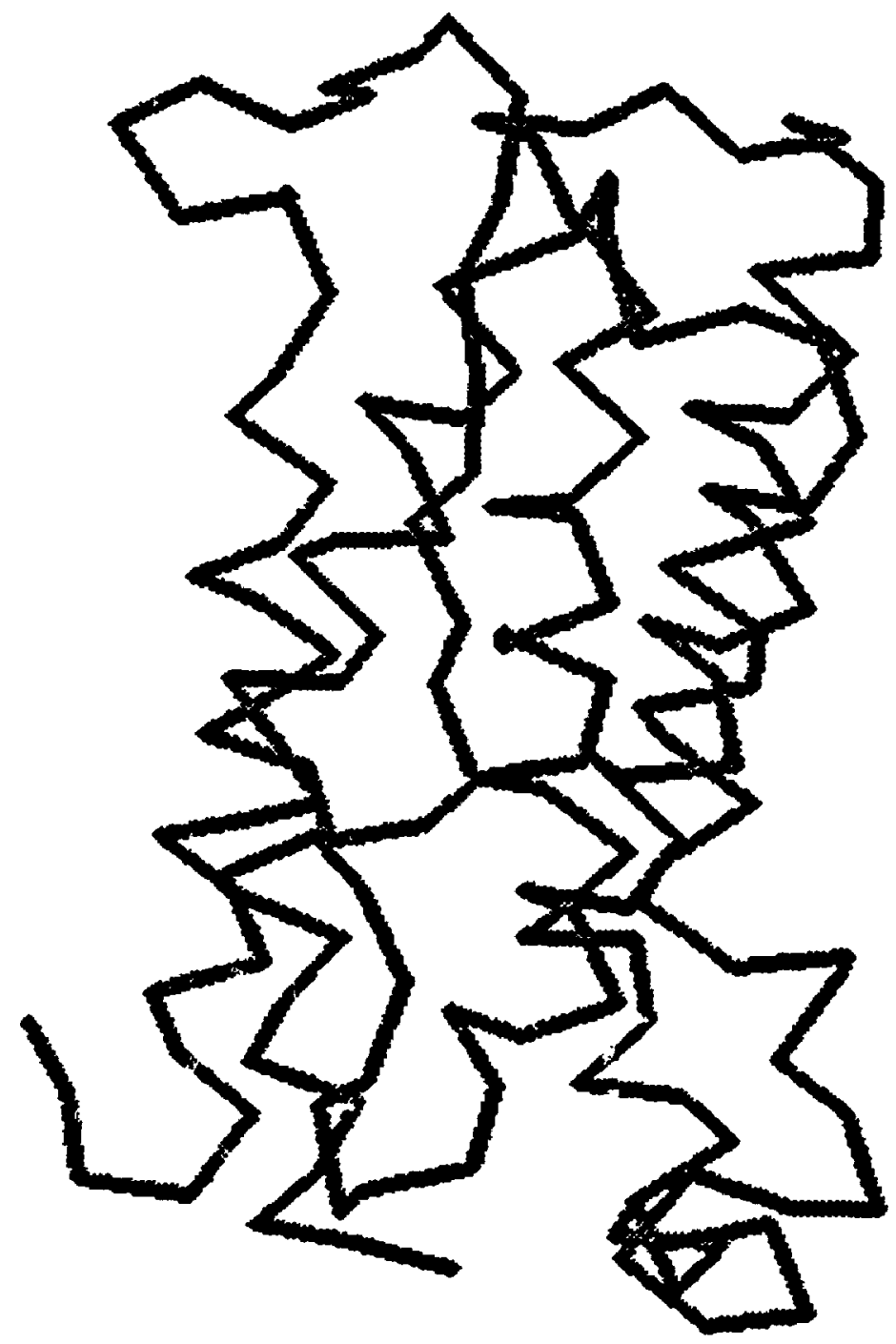

Figure 2.2.5. Human interferon-alpha 2 protein, backbone trace diagram (Murgolo et. al., 1993) 
The genetic code is the relationship between the sequence of nucleotides in DNA (or mRNA transcript) and the sequence of amino acids in the corresponding protein. The genetic code uses genetic information in the sequence of nucleotides in a DNA molecule to specify the sequence of amino acids in a protein. During protein synthesis, the coded information in DNA is initially transmitted to mRNA, i.e. the deoxyribonucleotide sequence is transcribed into a complementary sequence of ribonucleotides. The mRNA is then trans!nted into one or more polypeptides, with each amino acid of a polypeptide being encoded for by a particular sequence of three nucieotides (codon) in the mRNA. An mRNA molecule contains a series of nonoverlapping nucleotide triplets (codons) which specify the amino acid sequence of a polypeptide. Polypeptide synthesis begins at a specific AUG (initiator codon) in the mRNA and the tanslating machinery reads the triplets sequentially from this point. Thus, although triplets in a sequence of nucleotides could be read in three different ways (reading frames), in practice the correct reading frame is determined by the fixed starting point.

A codon does not interact directly with its corresponding amino acid. The amino acid must be linked to a tRNA adaptor molecule which contains a triplet of nucleotides (anticodon) complementary to the codon for that amino acid. For example, codon ACG is recognized by a tRNA containing the anticodon CGU. Codons and anticodons are written in the 5'-to-3' direction. The first nucleotide in a codon pairs with the third nucleotide in its corresponding anticodon.

The four nucleotides (A,C,G and $U$ ) when grouped into triplets in an mRNA molecule can generate $4^{3}=64$ possible triflet combınations. 61 codons encode for 20 amino acids. In most cases an amino acid is encoded by multiple (synonymous) codons. This phenomenon is referred to as degeneracy. The remaining three codons, UAA (ochre codon), UAG (umber codon) and UGA (opal or umber codon), are called nonsense codons (stop codons) (termination codons) which specify the termination of polypeptide chain synthesi: 
The three bases of a codon do not contribute equally to the specificity of the codon. The second base contributes maximally and the third base contributes minimally. Thus, certain codons which specify the same amino acid differ from each other only in the third base. This phenomenon is referred to as the wobble hypothesis.

The genetic code is nearly universal, i.e. applicable to all living systems. This phenomenon is referred to as the universal code theory. There are exceptions, e.g. in mammalian mitochondria AUA specifies methionine, while AGA and AGG function as stop codons. Mitochondria have a different genetic code because mitochondrial DNA uses a distinct set of tRNAs. Thus, the genetic code is nearly but not ahoolutely universal. It is believed that distinct genetic codes evolved from a common primordial genetic code (Singleton and Sainsbury, 1987). The genetic code is presented (Table 2.2.1.).

Codon

Amino Acid

AAA lysine

AAC asparagine

AAG lysine

AAU asparagine

ACA threonine

ACC threonine

ACG threonine

ACU threonine

AGA arginine

AGC serine

AGG arginine

AGU serine

AUA isoleucine

AUC isoleucine

AUG methionine

AUU isoleucine

CAA glutamine

CAC histidine

CAG glutamine

CAU histidine

CCA proline

CCC proline

CCG proline

CCU proline

CGA arginine

CGC arginine

CGG arginine

CGU arginine

CUA leucine 


$\begin{array}{ll}\text { CUC } & \text { leucine } \\ \text { CUG } & \text { leucine } \\ \text { CUU } & \text { leucine } \\ \text { GAA } & \text { glutamic acid } \\ \text { GAC } & \text { aspartic acid } \\ \text { GAG } & \text { glutamic acid } \\ \text { GAU } & \text { aspartic acid } \\ \text { GCA } & \text { alanine } \\ \text { GCC } & \text { alanine } \\ \text { GCG } & \text { alanine } \\ \text { GCU } & \text { alanine } \\ \text { GGA } & \text { glycine } \\ \text { GGC } & \text { glycine } \\ \text { GGG } & \text { glycine } \\ \text { GGU } & \text { glycine } \\ \text { GUA } & \text { valine } \\ \text { GUC } & \text { valine } \\ \text { GUG } & \text { valine } \\ \text { GUU } & \text { valine } \\ \text { UAA } & \text { stop } \\ \text { UAC } & \text { tyrosine } \\ \text { UAG } & \text { stop } \\ \text { UAU } & \text { tyrosine } \\ \text { UCA } & \text { serine } \\ \text { UCC } & \text { serine } \\ \text { UCG } & \text { serine } \\ \text { UCU } & \text { serine } \\ \text { UGA } & \text { stop } \\ \text { UGC } & \text { cysteine } \\ \text { UGG } & \text { tryptophan } \\ \text { UGU } & \text { cysteine } \\ \text { UUA } & \text { leucine } \\ \text { UUC } & \text { phenylalanine } \\ \text { UUG } & \text { leucine } \\ \text { UUU } & \text { phenylalanine } \\ & \text { senting }\end{array}$

Table 2.2.1. Genetic code (Suzuki et. al., 1989).

The three-dimensional structure of proteins has been reviewed (Richardson and Richardson, 1989) (Rizo and Gierasch, 1992). 


\subsection{Restriction Endonucleases}

Restriction endonucleases are strain-specific enzymes which enable bacteria to recognize and rapidly destroy foreign DNA. Restriction endonucleases are endodeoxyribonucleases that recognize specific double-stranded DNA sequences and cleave both strands. A restriction-modification system is a pair of enzymes consisting of a restriction endonuclease (restriction enzyme) and a methylase (modication enzyme). A methylase recognizes and modifies the same DNA sequence recognized by its corresponding restriction endonuclease. Methylases transfer methyl groups from Sadenosyl-methionine (AdoMet) to specific adenine or cytosine residues in the DNA. Modification enzymes protect host DNA against their own restriction endonucleases. Restriction-modificarion systems are found throughout bacteria and function to eliminate foreign DNA that gains entrance to the cell.

The restriction endonuclease nomenclature system consists of three components: 1 . an italicized three-letter acronym identifies the genus and species of the parent organism, 2. an additional letter, if necessary, identifies the strain or serotype and 3. a Roman numeral reflects the chronological order of identification or characterization. For example, EcoRI designates the first restriction endonuclease that was isolated from Escherichiz coli strain $\mathbf{R}$ (Smith and Nathans, 1973).

Three categories of restriction-modification systems have been defined on the basis of DNA cleavage. In type I systems, DNA cleavage occurs at nonspecific sites $400-7000$ nucleotides from the recognition site. In type II systems, DNA cleavage occurs at specific sites within or adjacent to the recognition site. In type II: systems, DNA cleavage occurs 25-27 nucleotides from the recognition site. Because of their specific recognition sites and the proximity of their cleavage sites, type II restriction endonucleases have been exploited as analytical tools in molecular biology. Therefore, the remainder of this section will focus on type II restriction endonucleases. 
Restriction endonuclease recognition sequences are usually composed of uniquelyspecified nucleotides. Degenerate recognition sequences also occur in which restriction endonucleases can accept several altemative nucleotides at a given position while rigorously excluding others. For example, the restrictior endonuclease AccI has the recognition sequence gtmkac. The degenerate nucleotide $m$ indicates that the enzyme can cleave the DNA sequence if either a or $\mathrm{c}$ nucleotides are present at the third residue. The restriction enzyme cannot cleave if $\mathbf{g}$ or $\mathbf{t}$ nucleotides are present. The degenerate nucleotide $\mathrm{k}$ indicates that the enzyme can cleave the DNA sequence if either $g$ or $t$ nucleotides are present at the fourth residue. The restriction enzyme cannot cleave if a or $\mathrm{c}$ nucleotides are present. Thus, at both central positions Accl is recognizing two specific base pairs in a specific orientation. The basis for this is unknown

Restriction endonuclease recognition sequences are usually 4-8 nucleotides long. Most recognition sites contain a dyad axis of symmetry. A symmetrical recognition sequence is termed a palindrome. A restriction endonuciease makes two single-standed breaks, one on each DNA strand at the recognition site. If the two breaks occur at the center of symmetry, the resulting termini are referred to as flush or blunt ends. If the two breaks are staggered, i.e. equidistant from and on opposite sides of the center of symmetry, the resulting termini are known as cohesive or sticky ends.

Restriction endonuclease cleavage usually occurs symmetrically within the recr znition sequence. Some endonucleases cleave on the $5^{5}$ side of the dyad axis producing overhanging 5' single-stranded termini, e.g. EcoRI (Hedgpeth et. al., 1972). Other endonucleases cleave in the center producing flush termini, e.g. Pvull (Gingeras et. al. 1981). Yet other endonucleases cleave on the 3' side producing overhanging 3' singlestranded termini, e.g. SacI (Arrand et. al., 1994). Restriction endonuclease cleavage patterns are presented (Table 2.3.1.). 


\begin{tabular}{|c|c|c|c|c|}
\hline Endonuclease & Recognition Sequence & Cleavage & lucts & Classificatio \\
\hline SacI & $\begin{array}{l}\text { GAGCTC } \\
\text { CTCGAG }\end{array}$ & $\stackrel{\text { GAGCT }}{\text { C }}$ & TCGAG & sticky ends \\
\hline PvuII & $\begin{array}{l}\text { CAGCTG } \\
\text { GTCGAC }\end{array}$ & $\begin{array}{l}\text { CAG } \\
\text { GTS }\end{array}$ & $\underset{\text { GAC }}{\text { CTG }}$ & blunt ends \\
\hline EcoRI & $\begin{array}{l}\text { GAATTC } \\
\text { CTTAAG }\end{array}$ & $\stackrel{\text { G }}{\text { CTTAA }}$ & $\begin{array}{r}\text { AATTC } \\
\mathbf{G}\end{array}$ & sticky en \\
\hline
\end{tabular}

Table 2.3.1. Restriction endonuclease cleavage patterns.

The property of staggered cleavage generates DNA fragments with protruding termini. Protruding termini are referred to as crhesive or sticky because such ends will form hydrogen bonds with complementary ends. DNA fragments produced by restriction endonuclease staggered cleavage readily anneal with other fragments produced by the sume restriction enzyme.

Consider DNA fragments that were generated by restriction endonuclease cleavage. DNA fragments originating from different sources can be joined together at their common restriction sites using the enzyme T4 DNA ligase. Protruding termini are readily ligated together. Blunt ends are more difficult to ligate than either type of protruding extension. The efficient joining of blunt-ended DNA fragments requires higher DNA fragment and T4 DNA ligase concentrations (Sgaramella, 1972).

The ability of resiriction endonucleases to cleave DNA at specific nucleotide sequences combined with the ability of T4 DNA ligase to rejoin phosphodiester backbone nicks in DNA permits the formation of recombinant DNA molecules. This ability to restructure DNA forms the basis of recombinant DNA techtology.

In addition to the creation and manipulation of recombinant DNA molecules, resiriction endonucleases are further used for the initial characterizution of DNA molecules. Using restriction endonucleases, it is possible to form a diagrammatic representation of the linear arrangement at which the restriction endonuclease cleavage sites occur within a DNA molecule. Such a diagram is referred to as a restriction map. Restriction endonuclease 
cleavage sites serve as useful reference points for allowing investigators to identify areas between unknown fragments and to target specific regions for nucleotide sequencing reactions.

Restriction endonucleases are used to identify potential genes in unmapped DNA. Nucleotide sequences in the promoter regions of numerous genes contain CG-rich regions that are identifiab'e by restriction endonucleases such as Hpall.

A total of 2558 restriction endonucleases are now known including 21 type I enzymes, 2532 type II enz:'mes and 5 type III enzymes (Macelis, 1994). There are 199 type II enzymes with prototype recognition sequences (Macelis, 1994). Numerous restriction endonucleases share common specificities. Restriction endonucleases with identical recognition sequences are termed isoschizomers. Restriction endonucleases with identical recognition sequences but different cleavage sites represent a restricted class of isoschizomers termed neoschizomers. For example, the SmaI and XmaI recognition sequences are $c c c^{*} g g g$ and $c^{*} c c g g g$ respectively, where the asterisk represents the cleavage site. Thus, the restriction endonucleases SmaI and XmaI are neoschizomers.

A comprehensive list of restriction endonucleases is compiled and broadcast monthly (Roberts and Macelis, 1994). Restriction endonucleases have been reviewed (Yuan, 1981) (Brooks, 1987) (Anderson, 1990) (Wilson and Murray, 1991) (Kant, 1992) (Roberts and Halford, 1993). 


\subsection{Transcription Factors}

Eukaryotic transcription is the synthesis of a messenger RNA (mRNA) molecule in which ribonucleotide 5'-triphosphates base-pair with complementary deoxyribonucleotides on a DNA template and are polymerized in the the $5^{\prime}-$ to-3 $^{\prime}$ direction. The transcription reaction is mediated by the enzyme RNA polymerase (transcriptase). Transcription has been reviewed (Weinmann, 1992) (Buratowski, 1994).

Gene expression is a multistage process which yields the formation of functional proteins. Transcription is the first stage of the gene expression process and results in the production of a primary mRNA transcript from genomic DNA. This is followed by the post-transcriptional processes of RNA spiicing and translation. Thus, transcription represents a primary target for gene regulation.

Transcription factors are a diverse class of DNA-binding proteins that bind to specific DNA sequences in gene regulatory regions and control gene transcription. DNAbinding proteins are classified into families on the basis of the protein structures used to mediate DNA binding or the dimerization that is often essential for DNA binding. Transcription factors are grouped into seven classes of related structural motifs: 1. helixturn-hslix, 2. homeodomain, 3. zinc finger, 4. steroid receptor, 5. leucine zipper, 6. helixloop-helix and 7. $\beta$-sheet (Pabo and Sauer, 1992).

Transcription factors bind to specific DNA recognition sites and exert two possible effects on gene expression: 1. transcriptional activation and 2. transcriptional repression.

1. $\because$ Inscriptional activation. Activation domains are specific regions of the transcription factor protein which activate transcription (Ptashne, 1988). Activation domains are distinct from the DNA binding regions of a transcription factor. Activation domains either interact with RNA polymerase directly or interact with other transcription factors in order to facilitate transcription. Transcription factors form a basal transcriptional complex that subsequently interacts with RNA polymerase. For example, transcription 
factor TFIID specifically binds to a DNA sequence referred to as a TATA box. The mammalian transcription factor ATF then binds to and changes the conformation of the transcription factor TFID. $T^{2}$ is altered conformation facilitates the subsequent binding of transcription factors TFIIC, TFIIE and RNA polymerase to form a stable transcriptional complex (Horikoshi et. al., 1988). In summary, transcription factor DNA-binding demains attach to DNA. Transcription factor activation domains alter the conformation of other transcription factors that have previously bound to DNA. This facilitates the assembly of a stable transcriptional complex.

2. Transcriptional repression. Transcription factors exert inhibitory effects on transcription by four mechanisms: 1 . competition for binding, 2. sequestration in solution, 3. quenching of activity and 4. direct repression.

1. Competition for binding. A negatively-acting transcription factor could act by binding to a DNA site and preventing the binding of a positively-acting transcription factor. For exampie, the $\beta$-interferon promoter has two transcriptional activators which are necessary for gene activation. A transcriptional repressor competes with the two activators for the same binding site. During viral infection the repressor is inactivated, allowing the two activators to bind thereby activating transcription (Goodbourn et. al., 1986).

2. Sequestration in solution. A negatively-acting transcription factor can interfere with DNA binding by interacting with a positively-acting transcription factor in solution and forming a complex which cannot bind to DNA. For example, the negatively-acting IPOU transcription factor interacts with the positively-acting CFla-POU transcription factor and prevents it from binding to DNA (Treacey et. al., 1991).

3. Quenching of activity. An inhibitory transcription factor can interfere with the activation of transcription mediated by a bound factor. For example, yeast miating type $\alpha_{2}$ protein binds to a site adjacent to the MCMI transcriptional activator site. The activation domain is masked thereby preventing the MCM1 from activating the $\alpha$-specific genss (Keleher et. al., 1988);. 
4. Direct repression. It is possible that transcription factors directly inhibit transcription using an inhibitory domain analogous to the activation domain. The inhibitory domain would interfere with the formation or stability of the basal transcriptional complex.

Gene expression is regulated in response to a particular signal or a particular cell type. Transcription factors are modulated by two mechanisms: 1 . regulation of synthesis and 2. regulation of activity.

1. Regulation of synthesis. Regulated synthesis results in a particular transcription facior being present in only a specific cell type. For example, the expression of the Oct-2 transcription factor gene results in the specific activation of immunoglobulin gene expression in B cells (Scheidereit et. al., 1987).

2. Regulation of activity. A transcription factor may be synthesized in all tissues but is converted into an active form only in a specific cell type or in response to a specific stimulus. Transcription factors may be activated by three mechanisms: 1. ligand binding, 2. dissociation of an inhibitory protein and 3. protein modification.

1. Ligand binding. A transcription factor when bound with a ligand undergoes conformational change resulting in activation. For example, yeast transcription factor ACE1 activates trarscription of the metallothionein gene in response to copper. The yeast transcription factor undergoes a conformational change in the presence of copper. This allows ACE1 to bind regulatory sites in the metallothionein gene and activate transcription (Furst et. al., 1988).

2. Dissociation of an inhibitory protein. A transcription factor may be activated by the disruption of an inhibitory protein-protein interaction. For example, the phorbol sster treatment of $\mathrm{T}$ cells or HeLa cells disrupts the interaction of an inhibitory protein with the NK-KB protein. Thus, phorbol ester treatment activates the NF-KB transcription factor (Baeurele and Baltimore, 1988).

3. Protein modification. Protein modification is a direct means of activating a transcription factor in response to a specific signal. For example, cyclic AMP stimulates 
the enzyme protein kinase $A$. Protein kinase $A$ then phosphorylates the CREB transcription factor. CREB subsequently mediates the activation of several genes (Yamamoto et. al., 1990).

Transcription factors have been reviewed (Morimoto, 1992) (Pabo and Sauer, 1992) (Latchman, 1993). 


\subsection{Interferon}

The cytokines are a broad heterogenous group of proteins that regulate intercellular communication. The cytokines are endogenous mediators of inflammatory and immunological host defense reactions. The cytokines have multiple regulatory roles: 1 . cell proliferation, 2. cell differentiation, 3. hematopoiesis, 4. immune response, 5. fever, 6. inflammatory response, 7. cytotoxic cells, 8. phagocytic cells, 9. tissue remodelling. 10. bone formation, 11. antiparasitic host defense and 12. antiviral host defense. Cytckine classes include: 1. growth factors, 2. lymphokines, 3. colony stimulating factor: 4 . transforming growth factors, 5. tumor necrosis factors and 6. interferons. Cytokine research will generate insight into the basic principles of cell biology and immunology. The cytokines are of further clinical significance. Thus, the cytokines have been the subject of intense investigation. Cytokine biochemistry has been reviewed (Evans and Whicher. 1993).

The interferons are a class of potent multifunctional cytokines that form a primary defense mechanism against viral infections and neoplasms. The interferons were origina!ly identified on the basis of their ability to inhibit viral replication. The interferons exhibit additional regulatory effects: 1 . cell proliferation, 2. cell differentiation, 3. immune system, 4. macrophage activation, 5. enhancement of lymphocyte-mediated toxicity and 6 . hormonal interactions. The interferons exert their multiple effects primarily by inducing protein synthesis. The interferons are present in numerous vertebrate species including: 1 . man, 2. mouse, 3. rat, 4. pig and 5. cattle. Interferon biochemistry has been reviewed (Sen and Lengyel, 1992).

Interferon classification is based on three parameters: 1. sensitivity to awid treatment, 2. antigenicity and 3. cellular origin. Sensitivity to acid treatment: type I and type II interferons are acid-stable and acid-labile respectively. Antigenicity: $\alpha, \beta, \omega$ and $\gamma$ interferons designate four serologically-distinct families. Cellular origin: leukocyte, 
fibroblast, and immune interferons correspond to the $\alpha, \beta$ and $\gamma$ serotypes respectively. The paper adopts the systematic interferon nomenclature of Diaz et. al. (1993) (Táble 2.5.1.).

Type Family

I alpha

beta

omega

non-alpha, non-omega

II ganma
Gene

interferon-alpha 1 gene

interferon-alpha 2 gene

interferon-alpha 4 gene

interferon-alpha 5 gene

interferon-alpha 6 gene

interferon-alpha 7 gene

interferon-alpha 8 gene

interferon-alpha 10 pseudogene

interferon-alpha 13 gene

interferon-alpha 14 gene

interferon-alpha 16 gene

interferon-alpha 17 gene

interferon-alpha 21 gene

interferon-alpha 22 pseudogene

interferon-beta 1 gene

interferon-omega 1 gene

interferon-omega 2 pseudogene

interferon-omega 4 pseudogene

interferon-omega 5 pseudogene

interferon-omega 9 pseudogene

interferon-omega 15 pseudogene

interferon-omega 18 pseudogene

interferon-omega 19 pseudogene

interferon-alpha 11 pseudogene (type y)

interferon-alpha 12 pseudogene (type $x$ )

interferon-alpha 20 pseudogene

interferon-alpha? pseudogene

interferon-gamma gene
Symbol

IFNAI

IFNA2

IFNA4

IFNA5

IFNA6

IFNA7

IFNA8

IFNA10

IFNA13

IFNA14

IFNA16

IFNA17

IFNA21

IFNAP22

IFNB 1

IFNW1

IFNWP2

IFNWP4

IFNWP5

IFNWP9

IFNWP15

IFNWP18

IFNWP19

IFNP11

IFNP12

IFNP20

IFNP23

Table 2.5.1. Nomenclature of the luman interferon genes (Diaz et. al., 1993).

Human interferon-alpha corresponds to an acid-stable, serologically-distinct family of cytokines produced by leukocytes. The human interferon-alpha family consists of twelve proteins: 1. IFNA1, 2. IFNA2, 3. IFNA4, 4. IFNA5, 5. IFNA6, 6. IFNA7, 7. IFNA8, 8. IFNA13, 9. IFNA14, 10. IFNA16, 11. IFNA17 and 12. IFNA21 (Diaz et. al.. 
1993). Human inteferon-alpha protein molecular weights range from 16,000 to 27,000 (Zoon et. al., 1987). Disulfide bonds are formed between the residues cys 1 - cys 98 and cys 29 - cys 138 in human inteferon-alpha 2 (Wetzel, 1981). Several human interferonalpha proteins are glycosylated (Labdon et. al., 1984). Human interferon-alpha has demonstrated significant activity in the treatment of several viral and oncological diseases: 1. hairy cell leukemia, 2. condyloma acuminata, 3. AIDS-related Kaposi's surcoma, 4. hepatitis B and 5. chronic hepatitis C (Dorr, 1993). Human interferon-alpha has been reviewed (Gutterman, 1994).

The human interferon-alpha multigene family consists of twelve genes and two pseudogenes. The twelve genes include: 1. IFNA1, 2. IFNA2, 3. IFNA4, 4. IFNA5, 5. IFNA6, 6. IFNA7, 7. IFNA8, 8. IFNA13, 9. IFNA14, 10. IFNA16, 11. IFNA17 and 12. IFNA21. The two pseudogenes include: 1. IFNA10 and 2. IFNAP22 (Diaz et. al., 1993). The human interferon-alpha genes and pseudogenes have been localized by in situ chromosomal hybridization to the 9p13 - 9p22 chromosomal bands (Figure 2.5.1.) (Trent et. al., 1982). A physical map of the human type-I interferon gene cluster has been constructed from YAC clones. The physical map demonstrates the location and relative orientation of the human interferon-alpha genes and pseudogenes (Figure 2.5.2.) (Diaz et. al., 1994). 

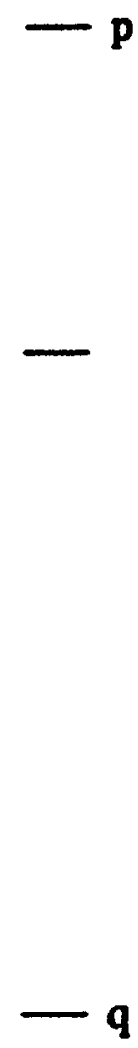

Figure 2.5.1. Human chromosome 9 cytogenetic map featuring type-I interferon gene cluster locus (Trent et. al., 1982) (Adler, 1994). 

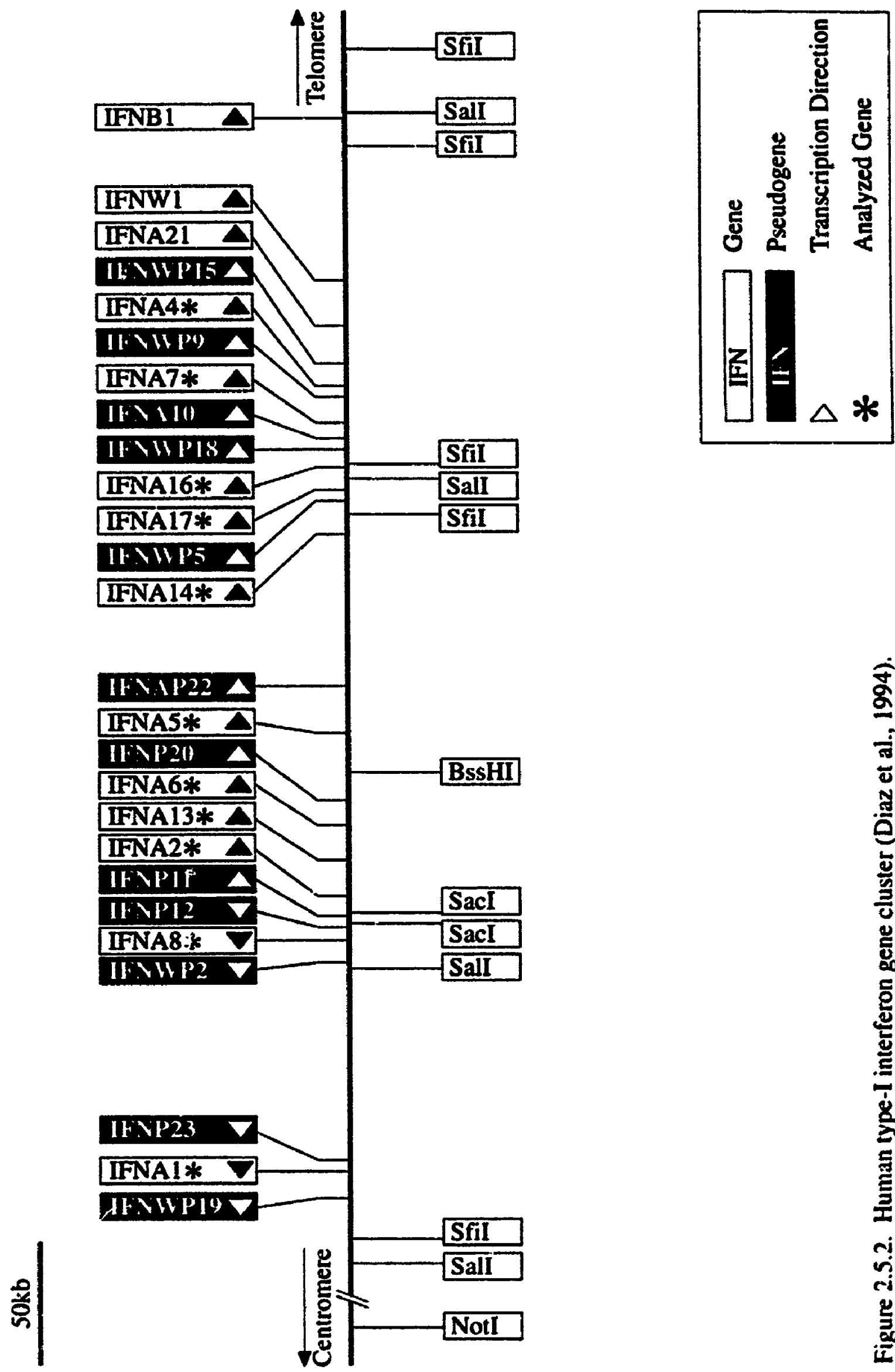
The human interferon-alpha genes lack introns (Henco et. al., 1985). A human interferon-alpha gene encodes a preinterferon protein that consists of a signal (leader) peptide and a mature peptide. The signal peptide facilitates interferon transport through the cellular membrane during secretion. During maturation the leader peptide is proteolytically cleaved from the preinterferon protein leaving the mature interferon protein. The signal peptide is composed of 23 amino acids. The mature interferon peptide is composed of 165 - 166 amino acids (Weissmann and Weber, 1986).

Human interferon-alpha genes are normally not expressed in uninduced cells. Human interferon-alpha induction occurs in response to three stimuli: 1. viruses, 2. double-stranded RNA in the form of poly (I)-poly (C) or 3. bacterial lipopolysaccharides (DeMaeyer and DeMaeyer-Guignard, 1988). Double-stranded RNA is the intermediate product responsible for viral-induced interferon synthesis (Lai and Joklik, 1973).

Current knowledge of human interferon-alpha transcriptional regulation is limited primarily to the human interferon-alpha 1 gene. The human interferon-alpha 1 gene is transcriptionally regulated (Weidle and Weissmarn, 1983). Viral-induced transcription is mediated by the 5 -promoter region of the human interferon-alpha 1 gene. The 5 flanking regions of the human interferon-alpha genes display a $75 \%$ degree of homology (Sehgal. 1982). The virus-response element is the minimal promoter requirement for conferring transient viral-induced transcription on heterologous promoter elements. The virusresponse element is the promoter sequence extending between positions -109 to -64 in the 5 direction relative to the cap site of the human interferon-alpha 1 gene. The virusresponse element is composed of two imperfect repeats: 1. RepA and 2. RepB. RepA contains two multiple-sequence alignment reg:ons termed R1 and R2. RepB similarly contains two multiple-sequence alignment regions termed R1' and R2' (Ryals et. al., 1985). 
The TG factor is a transcriptional activator of human interferon-alpha. The TG factor recognition site is referred to as the TG sequence. One TG seque-se has been identifier in the human interferon-alpha 1 promoter (MacDonald et. al., 1990).

The interferon regulatory factors are transcriptional regulators of human interferonalpha and human interferon-beta. Two interferon regulatory factors have been identified: interferon regulatory factor 1 (IRF-1) and interferon regulatory factor 2 (IRF-2). Interferon regulatory factor 1 recognizes sequences in both human interferon-alpha and human interferon-beta promoters (Fujita et. al., 1988) (Miyamoto et. al., 1988). Interferon regulatory factors 1 and 2 are structurally related and recognize the same binding sequences. However, these factors have opposite effects: interferon regulatory factor 1 is a transcriptional activator whereas interferon regulatory factor $\mathbf{2}$ is a transcriptional repressor (Harada et. al., 1989). Two interferon regulatory factor-like sites were identified in the human interferon-alpha 1 promoter (MacDonald et. al., 1990).

In summary, current knowledge of human interferon-alpha transcriptional regulation is limited primarily to the human interieron-alpha 1 gene. The promoters of the remaining human interferon-alpha genes are less well characterized than the human interferon-alpha 1 promoter. The current research objective is the characterization of the human interferon-alpha promoters. Identification of promoter transcription factor binding sites will facilitate elucidation of the transcriptional mechanism by which the human interferon-alpha genes are expressed. Human interferon-alpha molecular genetics has been reviewed (Henco et. al., 1985) (Weissmann and Weber, 1986) (Hayes and Zoon, 1994). 


\subsection{Retinoblastoma}

Retinoblastoma $(\mathbf{R b})$ is a malignant intraocular tumor of the develvping retina that occurs in early childhood (Shields, 1983). The term retinoblastoma originated from the resemblance of tumor cells to undifferentiated retinoblasts (Gallie et. al., 1982). Retinoblastoma is correlated with the inactivation of both alleles of the retinoblastoma (Rb1) gene. The retinoblastoma gene is usually inactivated by somatic mutation. Retinoblastoma gene failure renders cells incapable of negative regulation of cell proliferation and ultimately !eads to neoplastic transformation.

Retinoblastoma gene dysfunction predisposes primarily to retinoblastoma. Retinoblastoma mutations have been extended iv numerous malignancies: 1. bladder carcinomas (Horowitz et. al., 1989), 2. small cell lung carcinomas (Harbour et. al., 1988), 3. prostate carcinomas (Bookstein et. al., 1990), 4. breast carcinomas (Lee et. al., 1988) and 5. primary leukemia (Furukawa et. al., 1991). These findings provided the first evidence that the loss of a gene is correlated with tumorigenesis. This gave rise to $t^{t}: e$ concept of a tumor suppressor gene which has a negative influence on the regulation of cell growth. Thus, the retinoblastoma gene is the prototype model for a tumor suppressor gene (Friend et. al., 1986).

The retinoblastoma gene has been mapped by detailed $k$ aryotypic analysis to the 13q14.2 chromosomal band (Bowcock et. al., 1991). The retinoblastoma gene spans 180,388 nucleotides of genomic DNA and consists of 27 exons (Toguchida et. al., 1993). The retinoblastoma promoter is highly rich in GC residues that constitute a CG island (Ford et. al., 1990). CCAAT and TATA motifs have not been located. Several potential binding sites for the transcription factors Sp1, ATF and E2/DRTF1 have been found (Zacksenhaus et. a! , 1993). Endogenous retinoblastoma mRNA expression was observed in nearly all tissues examined. Ubiquitous expression of retinoblastoma mRNA is consistent with the promoter being a housekeeping promoter (Goodrich and Lee, 1993). The retinoblastoma 
mRNA (Rt 1 mRNA) is $4.7 \mathrm{~kb}$ long and consists of the regions: 1 . short 5 untransluted sequence, 2. $2.7 \mathrm{~kb}$ coding region and $3.2 \mathrm{~kb} 3^{\prime}$ untranslated sequence (Lee et. al., 1987a).

The retinoblastoma protein ( $\mathrm{pl} 10^{\mathrm{Rb}}$ ) is a $110-\mathrm{kD}$ nuclear phosphoprotein. The retinoblastoma protein consists of 928 amino acids and exhibits DNA-binding activity (Lee et. al., 1987b). The retinoblastoma protein is a transcription factor that regulates cell proliferation by modulating the activity of other transcriptional activators such as SpI. ATF2 and E2F/DRTF1 (Zacksenhaus et. al., 1993).

Retinoblastoma oncogenic mutations have been classified into four categories: 1 . deletion, 2. frameshift, 3. chain termination and 4. point (Zhang et. al., 1993). Chromosomal deletion mutations lead to a complete loss of function. Frameshift mutations and chain termination mutations lead to premature termination of translation leading to truncated or unstable retinoblastoma proteins (Dunn et. al.. 1989). Point mutations produce missense mutant retinoblastoma proteins (Gallie et. al., 1990). Point mutations affect RNA splicing donor or acceptor sequences and cause entire exons to be deleted from the processed retinoblastoma mRNA (Dunn et. al., 1989). Point mutations have been found in the potential Spl and ATF recognition sites in the retinobiastoma promoter (Sakai et. al., 1991). This suggests that these transcription factors are required for activating retinoblastoma transcription. Mutation $i$.alysis indicates that iwo conserved regions of the retinoblastoma protein, termed A and B, between amino acid residues 393 - 572 and 646 772 form a binding site for viral oncoprotein interactions (Hu et. al., 1990).

Retinoblastoma molecular genetics has been further reviewed (Weinberg. 1992) (Goodrich and Lee, 1993) (Levine, 1993) (Zacksenhaus et. al., 1993) (Zhang et. al., 1993). 


\subsection{Human Genome Project}

The human genome is the complete DNA sequence of Homo sapiens containing its entire genetic information. The diploid human genome is organized linearly into 23 pairs of chromosomes. A eukaryotic chromosome is a cytological structure located in the nucleus of a eukaryotic cell. Chromosomes are large double-helical DNA molecules that are associated with RNA and histone proteins. The DNA component is comprised of genes which function in the storage and transmission of genetic information. A genome is divided into a number of chromosomes that is characteristic for each species.

The diploid hurran genome contains $6-7$ billion base pairs of DNA which specify an estimated 100,000 genes (Guyer and Collins, 1993). A gene is a DNA proteinencoding sequence and its associated regulatory sequences. The coding nucleotides are transcribed to a messenger RNA (mRNA) molecule which is then translated to a protein molecule. A gene occupies a specific locus along a chromosome.

The 100,000 human genes encode, an equal number of proteins. The proteins control all aspects that comprise a functional organism, i.e. reproduction, development and metabolism. Currently, the function of only a small number of genes is known. The systematic characterization of all the genes comprising the human genome will have an enormous impact on medicine. Human genome structure has been reviewed (Wagner et. al., 1993).

The Human Genome Project (HGP) is an international research initiative with the goal of producing detailed genetic and physical maps for each of the 24 different human chromosomes. DNA sequence determination of the human genome will proceed when sequencing technology advances to the point at which it is economically feasible. Several nonhuman model organism genomes will be further analyzed. The model organism genomes will provide comparative information essential for understanding how the human genome functions. 
A genetic map depicts the linear arrangement of genes or genetic marker sites along a chromosome. Two types of genetic maps are identified: 1. genetic linkage maps and 2. physical maps. Genetic linkage maps are based on the frequency with which genetic markers are coinherited. The centimorgan (cM) is cue distance unit in genetic linkage maps. $1 \mathrm{cM}$ is equivalent to approximately 1 million base pairs or 1 megabase (Mb). Physical maps determine actual distances between genes on a chromosome. Physici. maps are based on collections of overlapping DNA fragments referred to as contigs. The base pair is the distance unit in physical maps. Either kilobase (kb) or megabase (Mb) units are used. A DNA sequence map in which the genetic code is specified by individual nucleotides, is the highest resolution physical map. Genetic maps serve as resources in the search for genes responsible for genetically-mediated diseases as well as for the further study of gene structure, function and expression.

The Human Genome Project has six scientific objectives: 1. genetic map, 2. physical map, 3. DNA sequence, 4. model organisms, 5. technology development and 6. computational tcols.

1. Genetic map. The creation of a dense ( $2 \mathrm{cM}$ resolution) genetic linkage map of the human genome. This map will require approximately 1650 evenly spaced and polymorphic markers to be characterized within the genome (Donis-Keller et. al., 1987). Such a map will facilitate the localization of genes exhibiting mendelian inheritance to within a tolerance of $1 \mathrm{Mb}$ (McKusick, 1994).

2. Physical map. The creation of a physical map of the human genome. This map will be formed by assembling contigs of at least $2 \mathrm{Mb}$ in length with sequence-tagged sites spaced every $100 \mathrm{~kb}$. The sequence-tagged site (STS) has been adopted as a genetic map definition language. A sequence-tagged site is a $100-1000$ nucleotide sequence with three characteristics: 1. unique in the genome, 2. identifies a mapped element and 3. amplifiable by the polymerase chain reaction. Sequence-tagged sites can be used to identify clones to 
generate overlapping inserts for a physical map. Sequence-tagged sites can also be used as correspondence points between genetic and physical maps (Olson et. al., 1989).

3. DNA sequence. Current genome analysis strategy is a two-tier approach in which maps are created first and wholesale sequencing will be delayed until technological improvements are made. Sequencing technology is being improved by two approaches: 1 . optimization of existing technologies and 2. development of novel technologies. Existing sequencing technology opimizations include: 1. improved experimental planning, 2 . automation and 3. increased data throughput. Novel sequencing technologies undergoing development include: 1. time-of-flight mass spectrometry (Jacobson et. al., 1991), 2. atomic force and scanning tunneling microscopies (Lindsay et. al., 1991), 3. single molecule sequencing (Soper et. al., 1991) and 4. hybridization (Drmanac et. al., 1990). Innovative sequencing technologies will offer order-of-magnitude improvements.

4. Model organisms. Parallel analysis of the genomes of a small number of wellcharacterized nonhuman model organisms offers two advantages: 1. model genomes are smaller and more tractable to analysis and 2. elucidation of model genomes will facilitate interpretation of the human genome. Nine organisms have been designated for genome analysis: 1. Mus musculus (mouse), 2. Caenorhabditis elegans (nematode), 3. Drosophila melanogaster (fruit fly), 4. Arabidopsis thaliana (plant), 5. Saccharomyces cerevisiae (yeast), 6. Escherichia coli (bacterium), 7. Bacillus subtilis (bacterium), 8. Mycobacterium tuberculosis (bacterium) and 9. Mycobacterium leprae (bacterium).

5. Technology development. Methods for genetic mapping, physical mapping and DNA sequencing will have to be substantially improved before the complete analysis of large genomes is feasible. Instrumentation technologies to automate mapping and sequencing procedures are vital to the success of the genome project. Automation and robotics cut across numerous areas of genome research and require particular attention.

6. Computational tools. The collection, organization, interpretation and dissemination of large amounts of complex mapping and sequencing data generated by the 
Human Genome Project will require algorithms, software, dutabases and operational infrastructure development. Genome informatics consists of three areas: 1. laboratory data management, 2. databases and 3. analytical tools. The Genome Data Base (GDB) is emerging as a major resource for genome research. The Genome Data Base stores human gene mapping data including DNA markers, map locations, genetic disease loci and bibliographic reference citations (Cuttichia et. al., 1993). Computational genome tools have three analytical roles: 1 . analyze the complex signals generated by data-acquisition devices, 2. extract meaningful information from molecular sequences and 3. visualize scientific data. Genome informatics has been reviewed (Smith, 1994).

The impact of genomic maps on locating disease genes is already evident. New disease genes are being found and reported at the rate of several per month (Guyer and Collins, 1993). Positional cloning is a technique that makes it possible to isolate genes from defined chromosomal locations (Collins, 1992). For example, the Kallmann syndrome is an X-linked disorder of the gonadal and olfactory functions. Genetic linkage mapping localized the Kallmann syndrome gene to the Xp22.3 chromosomal band (Meitinger et. al., 1990). The Kallmann syndrome gene has been isolated (Franco et. al., 1991) (Legouis et. al., 1991).

The Human Genome Project has been further reviewed (Yager et. al., 1991) (Collins and Galas, 1993) (Engel, 1993) (Guyer and Collins, 1993) (Olson, 1993) (Hoffman, 1994). 


\section{Chapter 3}

\section{Nucleotide Motif Search Algorithm}

Numerous sequence analysis problems in computational molecular biology can be expressed is nucleotide motif search problems, e.g. restriction analysis and transcription analysis. Nucleotide motif search is a specialized instance of the multiple keyword search problem. A multiple keyword search algorithm was developed for searching for class 1 nucleotide motifs within DNA sequences.

\subsection{Keyword Search Problem}

Consider the keyword $k$ and the text $t$ to be two strings defined over an arbitrary alphabet $\Sigma$. The keyword length is defined as $m,|k|=m$. The text length is defined as $n$, $|t|=n$. The keyword length $m$ is typically much shorter than the text length $n, m \leq n$. The keyword search problem is to find all occurrences of the keyword as a substring within the text. The output is the set of positions in the text where the keyword occurs.

The multiple keyword search problem is a generalization of the keyword search problem. Consider a finite set of $y$ keywords $k_{1}, k_{2}, \ldots k_{y}$ and a text $t$ defined as strings over an arbitrary alphabet $\Sigma$. The multiple keyword search problem is to find all occurrences of each keyword as a substring within the text. The output is the set of positions in the text where any of the keywords occur. The multiple keyword search problem hac been solved using deterministic finite automata (Aho and Corasick, 1975). String processing algorithras have been reviewed (Aho, 1990). 


\subsection{Nı:cleotide Motif Search Problem}

A motif is defined as a continuous segment of a macromolecular sequence. Several motif classes have been defined including: 1. exact match, 2. percentage match, 3 . weighted matrix match, 4. inverted repeat or stem-loep, 5. direct repeat or 6. set membership (Tabie 3.2.1.). The simplest category is the class 1 motif which involves an exact match to a short defined sequence (Staden, 1990). Class 1 nucleotide motifs occur within DNA as transcription factor binding sites and restriction endonuclease recognition sites.

Class Description

1 Exact match to a short defined sequence. The IUB symbols can be used for DNA sequences (FEBS, 1985).

2 Percentage match to a defined short sequence. In nucleic acids, the IUB symbols can be used.

3 Match to a defined sequence, using a score matrix and a cutoff score. The DNA matrix gives scores to IUB symbols depending on the amount of overlap between them (Staden, 1988). MDM78 is used for proteins (Dayhoff et. al., 1978).

4 Match to a weight matrix. Scores can be added or multiplied (by adding their algorithms).

5 As in class 4 but on the complementary strand.

6 Inverted repeat or stem-loop. Fixed stem length, range of loop sizes and cutoff score using A-T, G-C $=2 ; \mathrm{G}-\mathrm{T}=1$.

$7 \quad$ Exact match to short sequence but with a defined step size.

8 Direct repeat. Fixed iepeat length, range of gap sizes and cutoff score. For protein sequences scores are cillculated using MDM78, but an identity matrix is used for nucleic acids.

9 Members of a set. A list of sets of allowed amino acids for each position in the motif. When they are first entered into the computer they can be typed in with the sets separated by commas. For example IVL,,,DEKR,FYWILVM defines a motif of length 5 residue positions in which one of $I, V$ or $L$ must be found in the first position, then anything in the next three; $D, E, K$ or $R$ in the fourth position and $F, Y, W, I, L, V$ or $M$ in the fifth. Alternatively the sets can be witten in the form of a weight matrix, which is how the program will store them. This class only applies to protein sequences because for nucleic acids "membership of a set" can be achieved using IUB symbols.

Table 3.2.1. Current list of motif classes (Staden, 1990). 
57

The nucleotide motif search problem may be stated as follows. Consider a finite set of $y$ class 1 nucleotide motifs $k_{1}, k_{2}, \ldots k_{y}$ and a DNA sequence $t$ defined as sequences over the alphabet $\Sigma_{D N A}=(A, C, G, T)$. The nucleotide motif search problem is to find all occurrences of each nucleotide motif as a subsequence within the DNA sequence. The output is the set of positions in the DNA where any of the nucleotide motifs occur.

In DNA sequence analysis, nucleotide motif search is a specialized instance of the multiple keyword search problem. Two DNA sequence analysis problems can be expressed as nucleotide motif search problems: 1 . restriction analysis and 2. transcription analysis. Thus, the identification of transcription factor binding sites and restriction endonuclease recognition sites within an arbitrary DNA sequence are specialized cases of the multiple keyword search problem. 


\subsection{Nucleotide Motif Search Algorithm}

A multiple keyword search algorithm was developed for searching for class 1 nucleotide motifs within DNA sequences. The serial class 1 nucleotide motif search algorithm is an adaptation of the Aho and Corasick (1975) algorithm.

The serial class 1 nucleotide motif search algorithm input consists of: 1 . a finite set of $y$ class 1 nucleotide motifs $k_{1}, k_{2}, \ldots$ ky defined as strings over the extended DNA alphabet $\Sigma_{\text {extended }}=(A, C, G, T, K, M, R, S, W, Y, B, D, H, V, N\}$ (Table 3.3.1.) and 2. a DNA sequence $t$ defined as a string over the basic DNA alphabet $\Sigma_{\text {basic }}=\{A, C, G, T\}$. The sum of the lengths of the $y$ nucleotide motifs is defined as $m, \sum_{i=1}^{y} \mid k i l=m$. The length of the DNA sequence is defined as $n,|t|=n$.

Symbol Nucleotides

$\begin{array}{ll}\mathbf{A} & \mathbf{A} \\ \mathbf{C} & \mathbf{C} \\ \mathbf{G} & \mathbf{G} \\ \mathbf{T} & \mathbf{T} \\ \mathbf{K} & \mathbf{G} \text { or } \mathbf{T} \\ \mathbf{M} & \mathbf{A} \text { or } \mathbf{C} \\ \mathbf{R} & \text { Aor } \mathbf{G} \\ \mathbf{S} & \mathbf{C} \text { or } \mathbf{G} \\ \mathbf{W} & \text { A or T } \\ \mathbf{Y} & \mathbf{C} \text { or } \mathbf{T} \\ \mathbf{B} & \mathbf{C} \text { or } \mathbf{G} \text { or } \mathbf{T} \\ \mathbf{D} & \text { Aor } \mathbf{G} \text { or } \mathbf{T} \\ \mathbf{H} & \text { A or } \mathbf{C} \text { or } \mathbf{T} \\ \mathbf{V} & \text { A or } \mathbf{C} \text { or } \mathbf{G} \\ \mathbf{N} & \text { A or } \mathbf{C} \text { or } \mathbf{G} \text { or } \mathbf{T}\end{array}$

Table 3.3.1. Summary of single-letter code nucleotide designations (FEBS, 1985).

The serial class 1 nucleotide motif search algorithm consists of two steps: 1 . automaton construction and 2. DNA sequence processing.

1. Automaton construction. The set of nucleotide motifs is used to construct a deterministic finite automaton. The deterministic finite automaton consists of a finite set of 
states $\mathbf{S}$ and a state-transition function s. Each state $s \varepsilon \boldsymbol{\Sigma}_{\text {extended. The finite states are }}$ constructed into a 15-ary tree graph with each state represented as a separate graph node. Each tree node has a maximum possible fanout of fifteen descendant nodes. A descendant pointer repiesents a state transition corresponding to one of the fifteen characters in the extended DNA alphabet.

For each state $s 1 \in S$ and input symbol $n \in \Sigma_{\text {basic, the state-transition function }}$ $\sigma\left(s_{1}, \mathrm{n}\right)$ produces another state $s_{2} \varepsilon \mathrm{S}$. The state-transition function $\sigma$ has two possible outcomes: 1. a forward state transition and 2. a failure state transition. In a forward state transition, the input symbol $\mathbf{n}$ matches an automaton node descendant. This causes the deterministic finite automaton to descend one level down the 15-ary tree. In a failure state transition, the input symbol does not match any of the automaton node descendants. In this case, the deterministic finite automaton is reas signed to the root node. Thus, the deterministic finite automatcn makes a state transition on each input symbol.

2. DNA sequence processing. The 15-ary tree root node is designated as the deterministic finite automaton start state. The DNA sequence is applied as input to the deterministic finite automaton. The deterministic finite automaton processes the DNA text by successively reading the DNA sequence nucleotides. The deterministic finite automaten makes a state transition on each DNA sequence nucleotide. When the deterministic finite automaton makes a transition to any of the designated accept states, a nucleotide motif has been traversed and the nucleotide motif position is output.

The serial class 1 nucleotide motif search algorithm output consists of a set of nucleotide motif positions. Each position indicates the leftmost boundary of a nucleotide motif that occurs as a subsequence within the DNA sequence. 


\section{Chapter 4}

\section{Intel iPSC/860 Hypercube Implementation}

The hypercube interconnection topology is introduced. The data-parallel programming model is further presented. These concepts are combined in the parallel adaptation of the serial class 1 nucleotide motif search algorithm for the hypercube architecture. The parallel class 1 nucleotide motif search algorithm is based on the Single Program Multiple Data programming paradigm.

\subsection{Hypercube Network}

A multiprocessor computer system is a parallel computer with two or more processors. Numerous topologies have been proposed for interconnecting the processors of a distributed multiprocessor computer system. A message-passing multiprocessor computer system based on the binary $\mathbf{k}$-dimensional hypercube interconnection topology was tirst proposed by Squire and Palais (1963). A binary k-dimensional hypercube interconnection network consists of $2^{k}$ vertices. The hypercube vertices each represent a processing node while the hypercube edges represent communication links between the processing nodes.

The processors communicate by passing messages along these links. A message sent from one processor to another may be routed through intermediate processors. The ordered list of processors visited by the message is a path. The length of a path is the number of links in the path.

The term hypercube refers to a binary k-dimensiona! hypercube interconnection topology. Hypercubes are synonymously known as boolean hypercubes, boolean ncubes, binary cubes or $Q_{n}$. A hypercube with $n$ nodes is constructed by numbering the 
nodes from 0 to $n-1$ and linking each pair of nodes whose binary representations differ by exactly one bit. Thus, a $k$-dimensional hypercibe has a set of $2^{k}$ functional nodes and links. Hypercube networks are illustrated (Figure 4.1.1):<smiles>[O]O[Ge]1OCOO1</smiles>
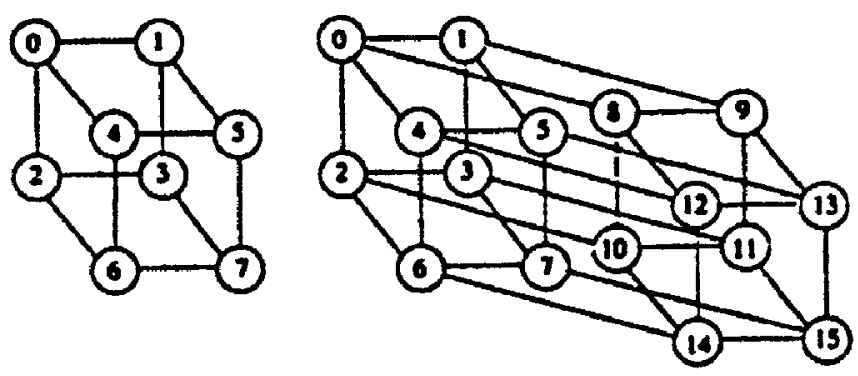

Figure 4.1.1. One-, two-, three- and four-dimensional hypercubes (Hatcher and Quinn, 1991).

The hypercube interconnection network has been the subject of significant research and commercial efforts. The hypercube has become a dominant topology because of its strong connectivity, regularity, symmetry and ability to embed other topologies (Saad and Schultz, 1988). The hypercube interconnection topology has formed the basis for viable multiprocessor computer systems, e.g. Intel iPSC/860 Hypercube (Intel Scientific Computers, 1990). The hypercube interconnection network and its associated algorithmic techniques have been further reviewed (Ranka and Sahni, 1990). 


\subsection{Data-Parallel Programming Model}

Parallel computation may be classified according to computational partitioning strategy. There are two computational partitioning methods: 1. data-parallelism and 2. ccntrol-parallelism (King et. al., 1990).

Data-parallelism refers to the use of multipie functional units to apply the same operation simultaneously to elements of a dataset. A clata-parallel computation divides data among the processors. Processors execute the same program but work on different data subsets. Data-parallel algorithms are appropriate for applications which perform the same set of operations repeatedly and independently on a large set of data. If there is no overhead associated with an increase in parallelism, then an $\mathbf{n}$-fold increase in the number of functional units should lead to an $\mathrm{n}$-fold increase in system throughput. Applications that feature nested loops to handle static and regular data structures are suitable cardidates for data-parallel decomposition, e.g. linear algebra operations (King et. al., 1990) (Quinn. 1994).

Control-parallelism is achieved through the simultaneous application of different operations to different data elements. A control-parallel computation decomposes an algorithm into modules of different functionality, which can be executed in parallel on multiple processors. Data flow between the processors can be arbitrarily complex. Control-parallelism is suitable for applications that use numerous independent subroutines. e.g. computer graphics simulations (King et. al., 1990) (Quinn, 1994).

Single Instruction Multiple Data (SIMD) computers are capable only of data parallelism. Multiple Instruction Multiple Data (MIMD) computers are capable of both data-parallelism and control-parallelism. Data-parallelism has two advantages over controlparallelism: 1. scalability and 2. simplicity. Data-paraliel algorithms are better able to scale to large numbers of processors. Data-parallel algoritıms have a single contro! flow which 
63

makes them easier to write and debug. Therefore, data-parallel implementations should be sought before control-parallel implementations are attempted (Quinn, 1994).

The Single Program Multiple Data (SPMD) paradigm refers to data-parallel implementations on MIMD architectures. SPMD parallelism arises from simultaneous operations across large datasets rather than from multiple threads of control. Thus, SPMD programs are easier to write and debug than arbitrary MIMD programs (Quinn, 1994).

Asynchronous algorithms have greater potential for errors such as deadlocks than synchronous algorithms. Hence the popularity of the SPMD programming style. SPMD programs characteristically resolve blocks of code by calls to message-passing routines. Processors execute identical blocks of code asynchronously using data stored in local memory. Data is then exchanged with other processors. Communication points further serve a synchronization function (Quinn and Hatcher, 1990). 


\subsection{Intel iPSC/860 Hypercube Implementation}

The serial class 1 nucleotide motif search algorithm has been converted into a parallel version. The parallel class 1 nucleotide motif search algorithm is an adaptation for the hypercube architecture (Saad and Schultz, 1988). The number of hypercube nodes present is p. Load balancing is achieved by partitioning the text string into the subtext strings $s_{1}, s_{2}, \ldots s q$. The number of subtext strings $q$ is the same as the number of hypercube processors $p, p=q$. The subtext length is defined as $r, n \bmod p=r$.

The parallel class 1 nucleotide motif search algorithm consists of three steps: 1 . host-to-node communication, 2. computation and 3. node-to-host communication. 1. Host-to-node communication. The host broadcasts the entire set of y class 1 nucleotide motifs to all hypercube nodes and routes one unique subtext to each hypercube node. 2. Computation. Within each hypercube node, the motif set and subtext are subjected to the serial class 1 nucleotide motif search algorithm to independently compute a set of nucleotide motif cccurrences. 3. Node-to-host communication. All hypercube nodes route their individual nucleotide motif occurrence sets back to the host.

The parallel class 1 nucleotide rnotif search algorithm uses a singl: thread of control and is written in Single Program Multiple Data (SPMD) programming style. The program uses a single thread of control and is loosely synchronous. Between the communication synchronization points, the processors asynchronously execute the same program but manipulate individual datasets. Thus, the parallel class 1 nucieotide motif search algorithm is an example of a data parallel algorithm. Data parallelism is defined as the simultaneous application of the same operation to elements of a dataset using multiple processors (Quinn, 1994).

The parallel class 1 nucleotide motif search algorithm operates in $O(m+(n / p))$ time and occupies $O(n+m p)$ space. 
The parallel class 1 nucleotide motif search algorithm was implemented on the Intel iPSC/860 Hypercube (Intel Scientific Computers, 1990). The algorithm was programmed in $\mathrm{C}$ language. The 15-ary tree data structure was programmed using recursion. 


\section{Chapter 5}

\section{Experimental Results}

Using the Intel iPSC/860 Hypercube implementation, tw dve luman genes were characterized by nucleotide motif search analysis. The genes are tabulated (Table 5.1.).

Gene

interferon-alpha 1 interferon-alpha 2 interferon-alpha 4 interferon-alpha 5 interferon-alpha 6 interferon-alpha 7 interferon-alpha 8 interferon-alpha 13 interferon-alpha 14 interferon-alpha 16 interferon-alpha 17 retinoblastoma
Location

appendix $\mathbf{D}$ appendix $\mathbf{E}$ appendix $F$ appendix $\mathbf{G}$ appendix $\mathrm{H}$ appendix I appendix $J$ appendix $\mathbf{K}$ appendix $\mathrm{L}$ appendix $M$ appendix $\mathbf{N}$

Table 5.1. Human genes analyzed by nucleotide motif search.

Nucleotide motif search analysis was performed using restriction endonuclease and transcription factor patterns. The restriction endonuclease and transcription factor patterns are tabulated (Table 5.2.).

Patterns

restriction endonucieases transcription factors human interferon transcription factors
Location appendix $A$ appendix B appendix C

Table 5.2. Restriction endonuclease and transcription factor paiterns.

Twelve types of genetic markers have been delineated on the human interferonalpha genes: 1. preinterferon peptide-encoding sequence, 2. signal peptide-encoding sequence, 3. mature interferon peptide-encoding sequence, 4. tata box, 5. virus-response 
element, 6. distal interferon regulatory factor site, 7. proximal interferon regulatory factor site, 8. TG sequence, 9. polyadenylation signal, 10. transcription initiation site, 11. transcription termination site and 12. restriction endonuclease cleavage sites.

A human interferon-alpha gene encodes a preinterferon protein that consists of a signal (leader) peptide and a mature peptide. The signal peptide facilitates interferon transport through the cellular membrane during secretion. During maturation the leader peptide is proteolytically cleaved from the preinterferon protein leaving the mature interferon protein. The preinterferon peptide-encoding sequence is translated into the preinterferon peptide which is made up of 188 - 189 amino acids. The signal peptideencoding sequence encodes the leader peptide which is comprised of 23 amino acids. The mature interferon peptide-encoding sequence directs the synthesis of the mature interferon peptide which is composed of 165 - 166 amino acids (Weissmann and Weber, 1986).

The tata box (TB) is a conserved AT-rich sequence commonly found in eukaryotic promoters. The tata box is the binding sequence recognized by transcription factor TFIID. Transcription factor TFIID is responsible for positioning RNA polymerase II at the transcription initiation site. The tata box is usually located -35 to -19 nucleotides $5^{\prime}$ relative to the transcription initiation site. The most common sequence is tataaaa. The tata box is otherwise known as the Hogness box after its discoverer D. Hogness.

The virus-response element (VRE) is the minimal promoter requirement ior conferring transient viral-induced transcription on heterologous promoter elements. The virus-response element $5^{\circ}$ boundary commences at -111 nucleotides $5^{\prime}$ relative to the transcription initiation site of the human interferon-alpha 1 gene. The human interferonalpha 1 virus-response element is 46 nucleotides long. The virus-response element sequence was determined to be gagtgcatgaaggaaagcaaaaacagaaatggaaagtggcccagaa. The virus-response element is composed of two imperfect repeats: 1. RepA and 2. RepB. RepA contains two multiple-sequence alignment regions termed R1 and R2. RepB 
similarly contains two multiple-sequence alignment regions termed R1' and R2' (Ryals et. al., 1985).

Interferon regulatory factors are transcriptional regulators of human interferonalpha and human interferon-beta. Two interferon regulatory factors have been identified: interferon regulatory factor 1 (IRF-i) and interferon regulatory factor 2 (IRF-2). Interferon regulatory factors 1 and 2 are structurally related. However, these factors have opposite effects: interferon regulatory factor 1 is a transcriptional activator whereas interferon regulatory factor 2 is a transcriptional repressor (Harada et. al., 1989). Interferon regulatory factor 1 recognizes sequences in both human interferon-alpha and human interferon-beta promoters (Fujita et. al., 1988) (Miyamoto et. al., 1988).

Harada et. al. (1989) claim that th. istinct transcription factors interferon regulatory factor 1 and interferon regulatory factor 2 bind to identical regulatory sequences. In binding site selection experiments, the interferon regulatory factor 1 and interferon regulatory factor 2 recognition sites were determined to be b(a)awa(n)y(r)amavn and s(a)waa(s)ygaaasb respectively (Tanaka et. al., 19:3).

The presence of two interferon regulatory factor binding sites within the human interferon-alpha 1 promoter was first proposed by MacDonald et. al. (1990). On the basis of their relative 5 distances from the transcription initiation site, the two sites are designated: 1. distal interferon regulatory factor site (DIRF) and 2. proximal interferon regulatory factor site (PIRF;.

Within human interferon-alpha 1 , the distal interferon regulatory factor site 5 boundary occurs at position -99 nucleotides relative to the cap site. The distal interferon regulatory factor site is 12 nucleotides long. The distal interferon regulatory factor site sequence is gaaagcauaaac. The proximal interferon regulatory factor site $5^{\prime}$ boundary occurs at position -58 nucleotides relative to the cap site. The proximal interferon regulatory factor site is 13 nucleotides long. The proximal interferon regulatory factor site sequence is gaangtggaaatc (MacDonald et. al., 1990). 
The TG factor (TG) is a transcription factor active in the transcriptional activation of the human interferon-alpha 1 gene. The TG sequence is located within the virusresponse element and is 12 nucleotides long. The TG sequence $5^{\circ}$ boundary occurs at position -85 nucleotides $5^{\prime}$ relative to the transcription iniation site. The TG sequence was determined to be gaaatggaaagt (MacDonald et. al., 1990).

Polyadenylation is the enzymatic addition of adenine nucleotides to the $3^{\prime}$ end of eukaryotic mRNA molecules. Polyadenylation is part of the processing that primary mRNA transcripts undergo subsequent to transcription and prior to transport from the nucleus to the cytoplasm. The polyadenylation tail refers to the segment of approximately 200 adenosine residues that is appended to the 3' end of the mRNA molecule. The polyadenylation tail has two possible functions: 1. mRNA stability and 2 . mRNA transport out of the nucleus. The polyarienylation signal is the hexanucleotide aataaa which plays an unknown role in polyadenylation. The polyadenylation signal occurs within the 3'-untranslated region of a eukaryotic gene and is located -20 to -18 nucleotides 5 relative to the polyadenylation tail.

Transcription refers to the synthesis of single-stranded mRNA from a doublestranded DNA template catalyzed by RNA polymerase. The mRNA is formed by complementary bass pairing to the DNA template. The transcription initiation site refers to the first DNA nucleotide to be transcribed to mRNA. Thus, the transcription initiation site corresponds to the 5 ' end of the mRNA molecule. The transcription initiation site is otherwise known as the cap site. The transcription termination site refers to the last DNA nucleotide to be transcribed to mRNA. Thus, the transcription initiation site corresponds to the $3^{\prime}$ end of the mRNA molecule.

A restriction endonuclease cleavage site is the specific nucleotide sequence in a double-stranded DNA recognized by a type II restriction endonuclease at which the enzyme cleaves the molecule. Restriction endonuclease recognition sites are usually 4 - 6 nucleotides long and exhibit bilateral symmetry, i.e. the recognition sites are pal: rdromes. 
The DNA strands may be cleaved opposite to one another to create blunt ends, e.g. Pvull. Alternately, the DNA strands may be cut in a staggered pattern to create sticky ends, e.g. EcoRI (Table 2.3.1.). 


\subsection{Human Interferon-Alpha 1 Gene}

The human interferon-alpha 1 genomic DNA nucleotide sequence has been determined. Biologisally-significant subsequences have been further elucidated: signal peptide, mature peptide, tata box, virus response element, distal interferon regulatory factor site, proximal interferon regulatory factor site, TG sequence, transcription initiation site and transcription termination site (Mantei et. al., 1980) (Nagata et. al., 1980) (Goeddel et. al., 1981) (Bowden et. al., 1984) (Ryals et. al., 1985) (MacDonald et. al., 1990). Human interferon-alpha 1 restriction analysis and transcription analysis data is presented (Tables 5.1.1. - 5.1.3.).

Data

nucleotide sequence restriction endonuclcase sites transcription factor sites

\section{Location}

appendix D.1

appendix D.2

table 5.1.2.

Table 5.1.1. Human interferon-alpha 1 restriction and transcription analysis data.

$\begin{array}{llr}\text { Eleinent } & \text { Sequence } & \text { Site } \\ \text { TB } & & \\ \text { VRE } & \text { tatttaingg } & 120 \\ \text { DIRF } & \text { gagtgcatgaaggaaagcaaaaacagaaatggaaagtggeccagaa } & 43 \\ \text { PIRF } & \text { gaaagcaaaaac } & 55 \\ \text { TG } & \text { gaaagtggaaatc } & 96 \\ & \text { gaaatggaaagt } & 68\end{array}$

Table 5.1.2. Human interferon-alpha 1 transcription control elements.

Parameter

nucleotides

restriction endonuclease sites

transcription factor sites

interferon transcription factor sites
Data

1179

187

157

173

Table 5.1.3. Human interferon-alp:ta 1 restriction and transcription analysis summary. 


\subsection{Human Interferon-Alpha 2 Gene}

The human interferon-alpha 2 genomic DNA nucleotide sequence has been determined. Biologically-significant subsequences have been further elucidated: signal peptide, mature neptide, tata box, virus response element, transcription initiation site and transcription termination site (Goeddel et. al., 1980) (Maeda et. al., 1980) (Streuli et. al.. 1980) (Goeddel et. al., 1981) (Lawn, et. al., 1981b) (Bowden et. al., 1984) (Ryals et. al.. 1985). Human interferon-alpia 2 restriction analysis and transcription analysis data is presented (Tables 5.2.1. - 5.2.3.).

Data

nucleotide sequence restriction endonuclease sites transcription factor sites
Location

appendix E.1 appendix E.2 table 5.2.2.

Table 5.2.1. Human interferon-alpha 2 restriction and transcription analysis data.

$\begin{array}{llr}\text { Element } & \text { Sequence } & \text { Site } \\ \text { TB } & \text { tattaagg } & 411 \\ \text { VRE } & \text { gagtgtataaagaaagcaaaaagagaagtagaaagtaacacaggg } & 335 \\ \text { DIRF } & \text { gaaagcaaaaag } & 346 \\ \text { PIRF } & \text { gaaaatgtaaacg } & 387 \\ \text { TG } & \text { gaagtagaaagt } & 359\end{array}$

Table 5.2.2. Human interferon-alpha 2 transcription control elements.

$\begin{array}{lr}\text { Parameter } & \text { Data } \\ & 1733 \\ \text { nucleotides } & 225 \\ \text { restriction endonuclease sites } & 182 \\ \text { transcription factor sites } & 195 \\ \text { interferon transcription factor sites } & \end{array}$

Table 5.2.3. Human interferon-alpha 2 restriction and transcription analysis summary. 


\subsection{Human Interferon-Alpha 4 Gene}

The human interferon-alpha 4 genomic DNA nucleotide sequence has been determined. Biologically-significant subsequences have been further elucidated: signal peptide, mature peptide, tata box, virus response element and transcription initiation site (Henco et. al., 1985) (Ryals et. al., 1985). Humañ interferon-alpha 4 restriction analysis and transcription analysis data is presented (Tables 5.3.1. - 5.3.3.).

Data

nucleotide sequence restriction endonuclease sites transcription factor sites

\section{Location}

appendix F.1

appendix F.2 table 5.3.2.

Table 5.3.1. Human interferon-alpha 4 restriction and transcription analysis data.

$\begin{array}{lll}\text { Element } & \text { Sequence } & \text { Site } \\ \text { TB } & \text { tatttaaga } & 697 \\ \text { VRE } & \text { gagtgcgtaaagaaagcaaaaacagagatagaaagtacaactagg } & 621 \\ \text { DIRF } & \text { gaaagcaaaaac } & 632 \\ \text { PIRF } & \text { gaaaatggaaatt } & 673 \\ \text { TG } & \text { gagatagaaagt } & 645\end{array}$

Table 5.3.2. Human interferon-:-ipha 4 transcription control elements.

Parameter

nucleotides

restriction endonuclease sites

transcription factor sites

interferor transcription factor sites
Data

2022

265

231

208

Table 5.3.3. Human interferon-alpha 4 restriction and transcription analysis summary. 


\subsection{Human Interferon-Alpha 5 Gene}

The human interferon-alpha 5 genomic DNA nucleotide sequence has been determined. Biologically-significant subsequences have been further elucidated: signal peptide, mature peptide, tata box, virus response element and transcription initiation site (Henco et. al., 1925) (Ryals et. al., 1985). Human interferon-alpha 5 restriction analysis and transcription analysis data is presented (Tables 5.4.1. - 5.4.3.).

Data Location

nucleotide sequence restriction endonuclease sites transcription factor sites appendix G.1 appendix G.2 table 5.4.2.

Table 5.4.1. Human interferon-alpha 5 restriction and transcription analysis data.

$\begin{array}{llr}\text { Element } & \text { Sequence } & \text { Site } \\ \text { TB } & \text { ttttaaga } & 337 \\ \text { VRE } & \text { aagagcataaaagaaagcaaaaagagaagtagaaagtaacacaagg } & 261 \\ \text { DIRF } & \text { gaaagcaaaaag } & 273 \\ \text { PIRF } & \text { gaaaatggaaact } & 313 \\ \text { TG } & \text { gaagtagaaagt } & 286\end{array}$

Table 5.4.2. Human interferon-alpha 5 transcription control elements.

Parameter

nucleotides

restriction endonuclease sites transcription factor sites

interferon transcription factor sites
Data

1475

183

166

179

Table 5.4.3. Human interferon-alpha 5 restriction and transcription analysis summary. 


\subsection{Human Interferon-Alpha 6 Gene}

The human interferon-alpha 6 genomic DNA nucleotide sequence has been determined. Biologically-significant subsequences have been further elucidated: signal peptide, mature peptide, tata box and transcription iritiation site (Henco et. al., 1985). Human interferon-alpha 6 restriction analysis and transcription analysis data is presented (Tables 5.5.1. - 5.5.3.).

Data

nucleotide sequence restriction endonuclease sites transcription factor sites
Location

appendix $\mathrm{H} .1$ appendix H.2 table 5.5.2.

Table 5.5.1. Human interferon-alpha 6 restriction and transcription analysis data.

$\begin{array}{lll}\text { Element } & \text { Sequence } & \text { Site } \\ \text { TB } & \text { tattraga } & 391 \\ \text { VRE } & \text { gagtaaataaatgaaagcaaaatcagacgtagaaagtaaattctgaa } & 323 \\ \text { DIRF } & \text { gaaagcaaaatc } & 335 \\ \text { PIRF } & \text { gaaaatggaaact } & 367 \\ \text { TG } & \text { gacgtagaaagt } & 348\end{array}$

Table 5.5.2. Human interferon-alpha 6 transcription control elements.

$\begin{array}{lr}\text { Prameter } & \text { Data } \\ \text { nucleotides } & 1544 \\ \text { restriction endonuclease sites } & 212 \\ \text { transcription factor sites } & 152 \\ \text { interferon transcription factor sites } & 183\end{array}$

Table 5.5.3. Human interferon-alpha 6 restriction and transcription analysis summary. 


\subsection{Human Interferon-Alpha 7 Gene}

The human interferon-alpha 7 genomic DNA nucleotide sequence has been determined. Biologically-significant subsequences have been further elucidated: signal peptide, mature peptide, tata box, virus response element and transcription initiation site (Henco et. al., 1985) (Ryals et. al., 1985). Human interferon-alpha 7 restriction analysis and transcription analysis data is presented (Tables 5.6.1. - 5.6.3.).

\section{Data}

nucleotide sequence restriction endonuclease sites transcription factor sites
Location

appendix I.1

appendix 1.2

table 5.6.2.

Table 5.6.1. Human interferon-alpha 7 restriction and transcription analysis data.

$\begin{array}{lll}\text { Element } & \text { Sequence } & \text { Site } \\ \text { TB } & \text { tattaaga } & 652 \\ \text { VRE } & \text { gagtgcataaagaaagcaaaaacagagatagaaagtaaaactagg } & 576 \\ \text { DIRF } & \text { gaaagcaaaaac } & 587 \\ \text { PIRF } & \text { gaaaatggaaatt } & 628 \\ \text { TG } & \text { gagatagaaagt } & 600\end{array}$

Table 5.6.2. Human interferon-alpha 7 transcription control elements.

\section{Parameter}

nucleotides restriction endonuclease sites transcription factor sites interferon transcription factor sites
Data

Table 5.6.3. Human interferon-alpha 7 restriction and transcription analysis summary. 


\subsection{Human Interferon-Alpha 8 Gene}

The human interferon-alpha 8 genomic DNA nucleotide sequence has been determined. Biologically-significant subsequences have been further elucidated: signal peptide and mature peptide (Henco et. al., 1985). The human inierferon-alpha 8 promoter region has not been sequenced. Therefore, it was not possible to determine the human interferon-alpha 8 transcription control elements. Human interferon-alpha 8 restriction analysis and transcription analysis data is presented (Tak'es 5.7.1. - 5.7.2.).

\section{Data}

nucleotide sequence

restriction endonuclease sites
Location

appendix J.1

appendix J.2

Table 5.7.1. Human interferon-alpha 8 restriction analysis data.

Parameter Data

nucleotides 633

restriction endonuclease sites $\quad 120$

transcription factor sites 73

interferon transcription factor sites $\quad 78$

Table 5.7.2. Human interferon-alpha 8 restriction and transcription analysis summary. 


\subsection{Human Interferon-Alpha 13 Gene}

The human interferon-alpha 13 genomic DNA nucleotide sequence has been determined. Biologically-significant subsequences have been further elucidated: peptide. tata box, virus response el $r .2 n t$ end transcription initiation site (Mantei et. al., 1980) (Todokoro, et. al., 1984) (Ryals et. al., 1985). Human interferon-alpha 13 restriction analysis and transcription analysis data is presented (Tables 5.8.1. - 5.8.3.).

Data

nucleotide sequence restriction endonuclease sites transcription factor sites

\section{Location}

appendix $\mathrm{K} .1$

appendix K.2 table 5.8.2.

Table 5.8.1. Human interfercn-alpha 13 restriction and transcription analysis data.

$\begin{array}{llr}\text { Element } & \text { Sequence } & \text { Site } \\ \text { TB } & \text { tattragg } & 119 \\ \text { VRE } & \text { gagttcaagaaggaaagcaaaaacagaaatggaaagtggtccagaa } & 42 \\ \text { DIRF } & \text { gaaagcaaaaac } & 54 \\ \text { PIRF } & \text { gaaag: 3gaaatc } & 95 \\ \text { TG } & \text { gaaatggaaagt } & 67\end{array}$

Table 5.8.2. Human interferon-alpha $1^{2}$ transcription control elements.

$\begin{array}{lr}\text { Parameter } & \text { Data } \\ \text { nucleotides } & 1108 \\ \text { restriction endonuclease sites } & 184 \\ \text { transcription factor sites } & 140 \\ \text { interferon taanscription factor sites } & 162\end{array}$

Table 5.8.3. Human interferon-alpha 13 restriction and transcription analysis summary. 


$$
2
$$




\subsection{Human Interferon-Alpha 14 Gene}

The human interferon-alpha 14 genomic. DNA nucleotide sequence has been determined. Biologically-significant subsequences have been further elucidated: signal peptide, mature peptide, tata box, virus response element and transcription initiation site (Henco et. al., 1985) (Ryals et. al., 1985). Human interferon-alpha 14 restriction analysis and transcription analysis data is presented (Tables 5.9.1. - 5.9.3.).

Data Location

nucleotide sequence restriction endoinu:ler se sites transcription facio: siles appendix L.1 appendix L.2 table 5.9.2.

Table 5.9.1. Human interferon-alpha 14 restriction and transcription analysis data.

Element

TB

VRE

DIRF

PIRF

TG

$$
\text { Sequence }
$$

tatttaaga caggacataaaggaaagccaaaagagaagtagaaaaaaacatga gaaagccaaaag gaaaatggaagct gaagtagaaa
Site

176

99

111

152

124

Table 5.9.2. Human interferon-alpha 14 transcription control elements.

$\begin{array}{lr}\text { Parameter } & \text { Data } \\ \text { nucleotides } & 1126 \\ \text { restriction endonuclease sites } & 160 \\ \text { transcription factor sites } & 133 \\ \text { interferon transcription factor sites } & 157\end{array}$

Table 5.9.3. Human interferon-alpha 14 restriction and transcription analysis summary. 


\subsection{Human Interferon-Alpha 16 Gene}

The human interforon-alpha 16 genomic DNA nucleotide sequence has been determined. Biologically-significant subsequences have been further elucidated: signal peptide, mature peptide, tata box and transcription initiation site (Henco et. al., 1985). Human interferon-alpha 16 restriction analysis and transcription analysis data is presented (Tables 5.10.1 - 5.10.3.).

Data

nucleotide sequence restriction endonuclease sites transcription factor sites
Location

appendix M.1

appendix M.2

table 5.10.2.

Table 5.10.1 Human interferon-alpha 16 restriction and transcription analysis data.

$\begin{array}{llr}\text { Element } & \text { Sequence } & \text { Site } \\ \text { TB } & & 385 \\ \text { VRE } & \text { tattaaga } & 309 \\ \text { DIRF } & \text { gagtgcataaagaaagcaaaaacagagatagaaagtaaaaccaggg } & 320 \\ \text { PIRF } & \text { gaaagcaaaaac } & 361 \\ \text { TG } & \text { gaaaatggaaatt } & 333\end{array}$

Table 5.10.2. Human interferon-alpha 16 transcription control elements.

$\begin{array}{lr}\text { Parameter } & \text { Data } \\ \text { nucleotides } & 1133 \\ \text { restriction endonuclease sites } & 148 \\ \text { transcription factor sites } & 123 \\ \text { interferon transcription factor sites } & 143\end{array}$

Table 5.10.3. Human interferon-alpha 16 restriction and transcription analysis summary. 


\subsection{Human Interferon-Alpha 17 Gene}

The human interferon-alpha 17 genomic DNA nucleotide sequence has been determined. Biologically-significant subsequences have been further elucidated: peptide, tata box, transcription initiation site and transcription termination site (Lawn et. al., 1981a). Human interferon-alpha 17 restriction analysis and transcription analysis data is presented (Tables 5.11 .1 - 5.11.3.).

Data

nucleotide sequence restriction endonuclease sites transcription factor sites
Location

appendix N.1 appendix $\mathbf{N} .2$ table 5.11.2.

Table 5.11.1 Human interferon-alpha : 7 restriction and transcription analysis data.

$\begin{array}{lll}\text { Element } & \text { Sequence } & \text { Site } \\ \text { TB } & & \\ \text { VRE } & \text { tatttaagg } & 539 \\ \text { DIRF } & \text { gagtgcaaaaagaaagcaaaaacagacatagaaagtaaaactagg } & 463 \\ \text { PIRF } & \text { gaaagcaaaaac } & 474 \\ \text { TG } & \text { gaaaatggaaatt } & 515 \\ \text { gacatagaaagt } & 487\end{array}$

Table 5.11.2. Human interferon-alpha 17 transcription control elements.

$\begin{array}{lr}\text { Parameter } & \text { Data } \\ \text { nucleotides } & 1873 \\ \text { restriction endonuclease sites } & 236 \\ \text { transcription factor sites } & 211 \\ \text { interferon transcription factor sites } & 182\end{array}$

Table 5.11.3. Human interferon-alpha 17 restriction and transcription analysis summary. 


\subsection{Human Retinoblastoma Susceptibility Gene}

The human retinoblastoma genomic DNA nucleotide sequence has been determined. The retinoblastoma susceptibility gene is composed of 180,388 nucleotides making it the longest known continous human DNA sequence (Toguchida et. al., 1993). Thus, the retinoblastoma susceptibility gene was used as an experimental subject in the nucleotide motif search parallel performance experiments. Human retinoblastoma restriction analysis and transcription analysis data is presented (Table 5.12.1.).

Parameter

nucleotides restriction endonuclease sites transcription factor sites interferon transcription factor sites
Data

180388

24699

20174

16662

Table 5.12.1. Human retinoblastoma restriction and transcription analysis summary. 


\section{Chapter 6}

\section{Genetic Analysis}

The nucleotide motif analysis is presented as four series of computational results: 1 . human interferon-alpha genetic maps, 2. human interferon-alpha promoter transcription maps, 3. human interferon-alpha promoter elements and 4. human interferon-alpha transcriotion regulatory mechanism.

\subsection{Human Interferori-Alpha Genetic Maps}

Human interferon-alpha genetic maps comprise the entire genes. The diagrams include the complete protein-encoding region as well as the nontranslated $5^{\prime}$ and $3^{\prime}$ flanking regions. The human interferon-alpha genetic maps indicate twelve types of genetic marker sites: 1. preinterferon peptide-encoding sequence (Peptide), 2. signal peptide-encoding sequence (Signal), 3. mature interferon peptide-encoding sequence (Mature Peptide), 4. tata box (Tata), 5. virus-response element (VRE), 6. distal interferon regulatory factor site (DIRF), 7. proximal interferon regulatory factor site (PIRF), 8. TG sequence (TG), 9. polyadenylation signal (PolyA), 10. transcription initiation site (mRNA>), 11. transcription termination site (mRNA<) and 12. restriction endonuclease cleavage sites (BsrI, EcokI, Mael, etc.). The human interferon-alpha genetic maps are presented (Figures 6.1.1. 6.1.11.). 

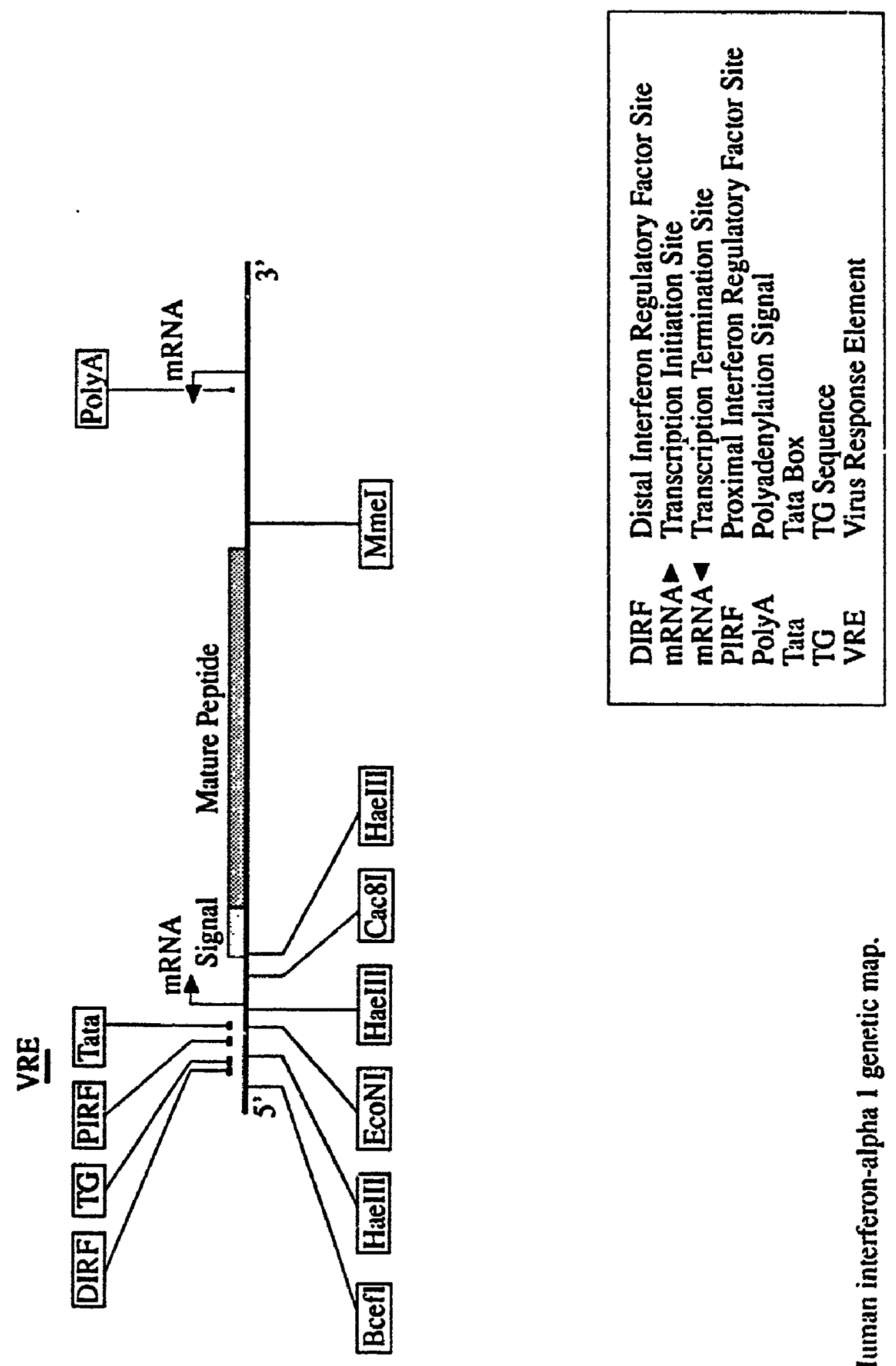

횜

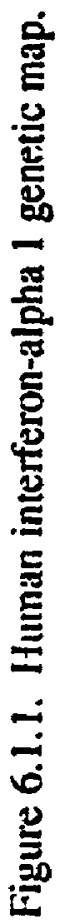



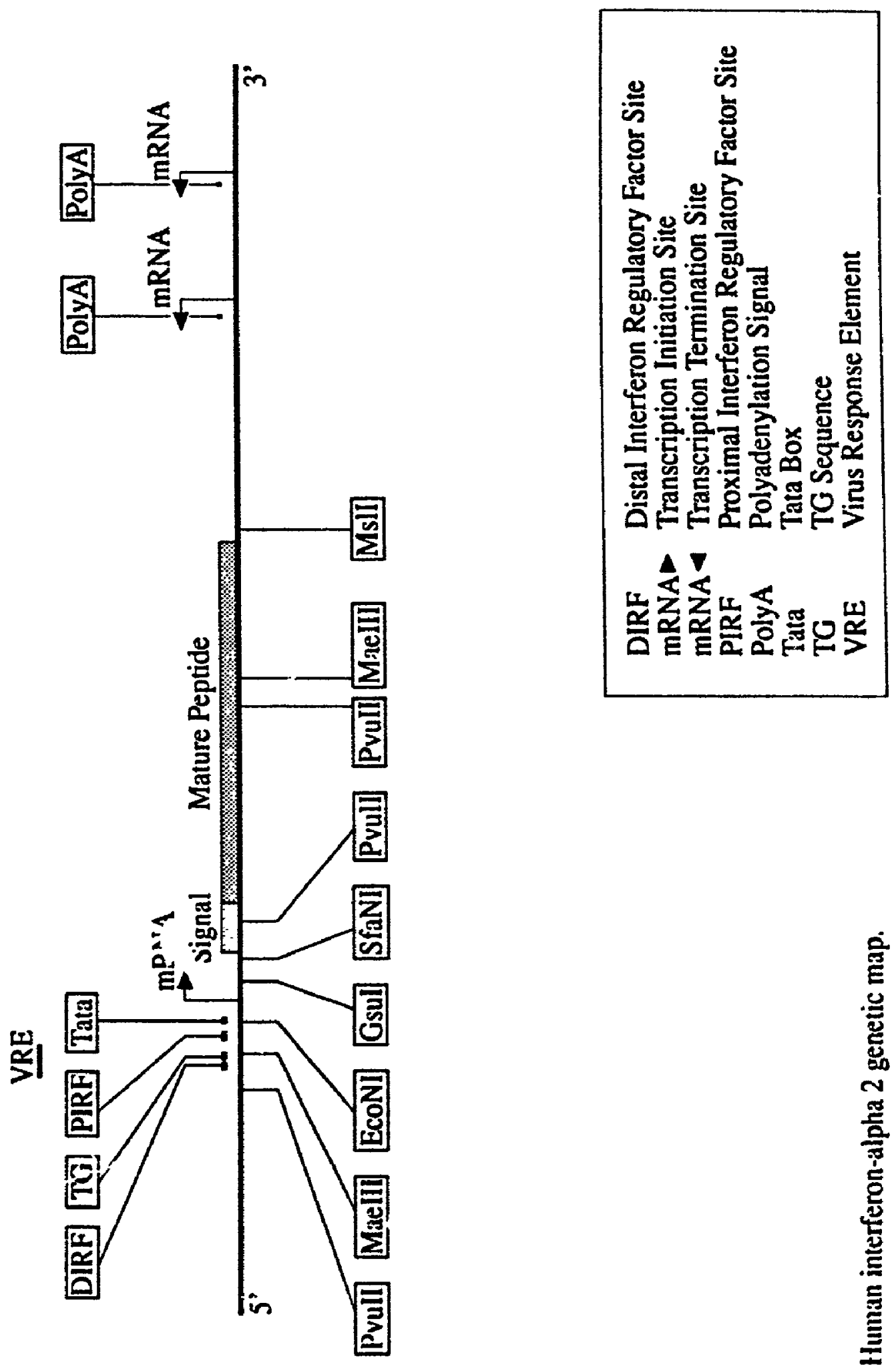

홍

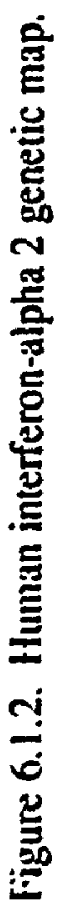



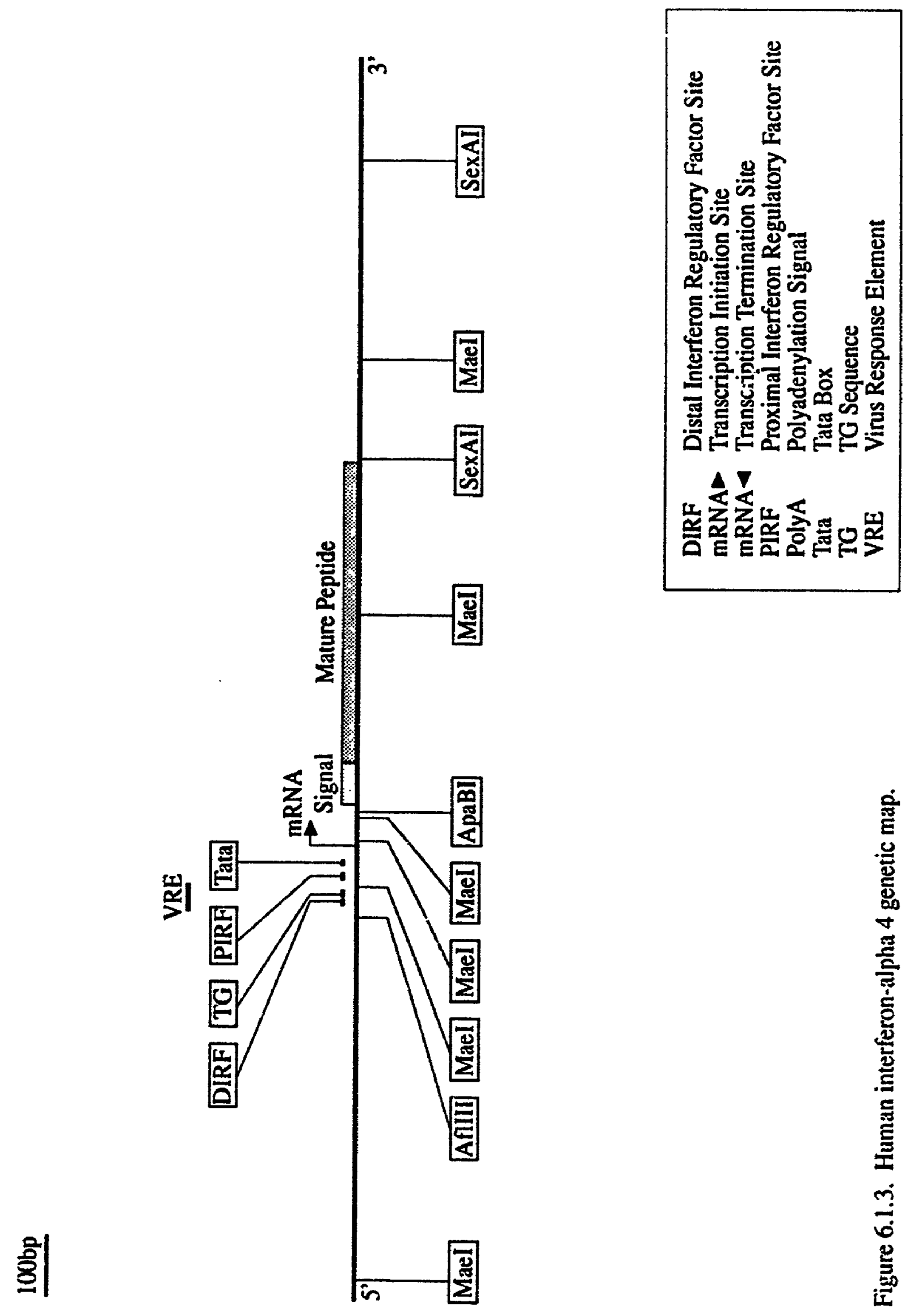

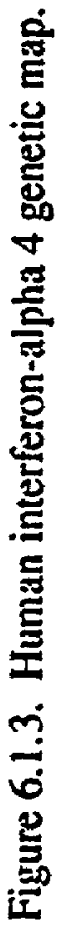



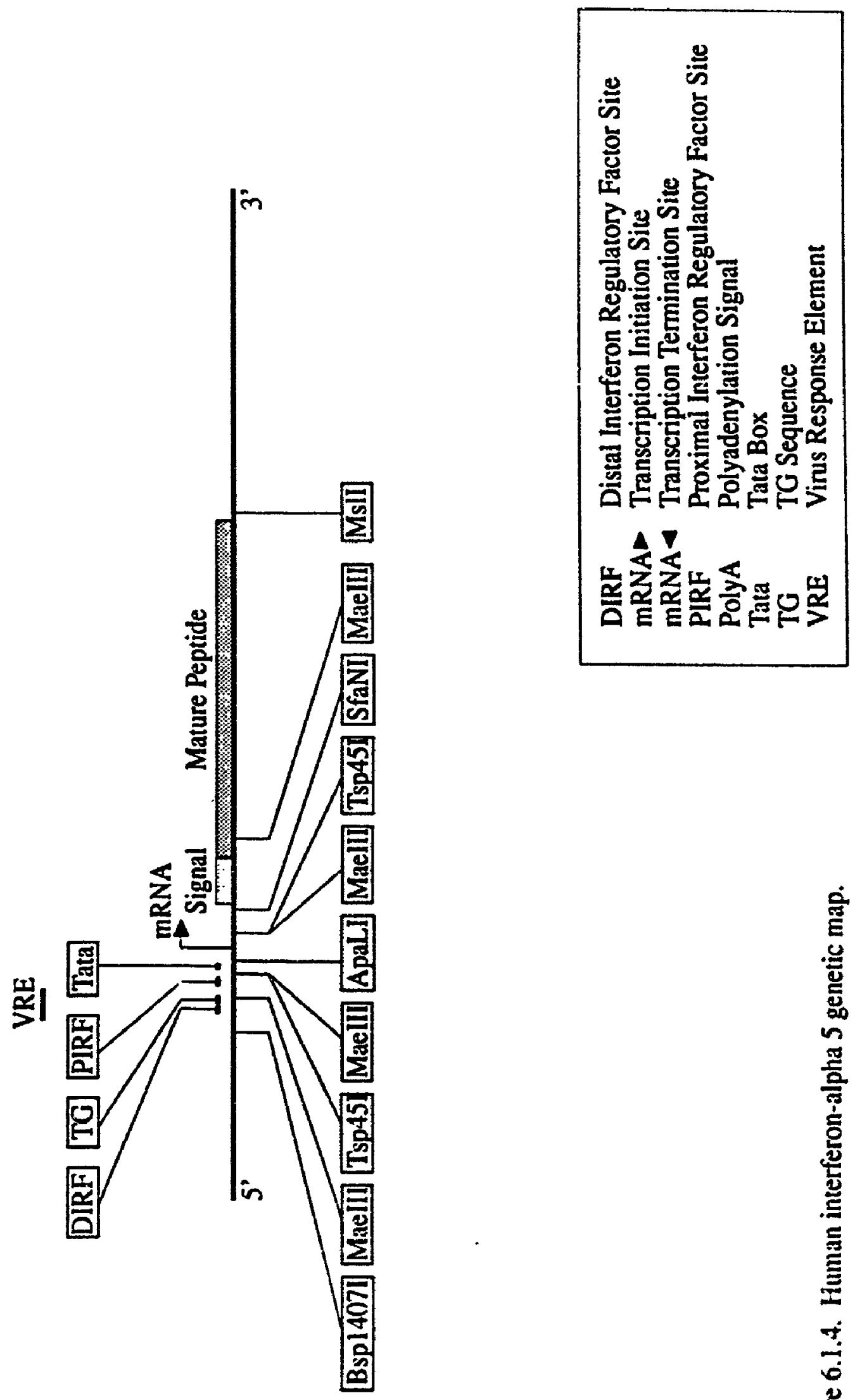

ㅎํ|

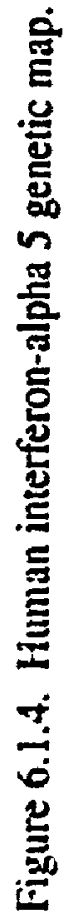



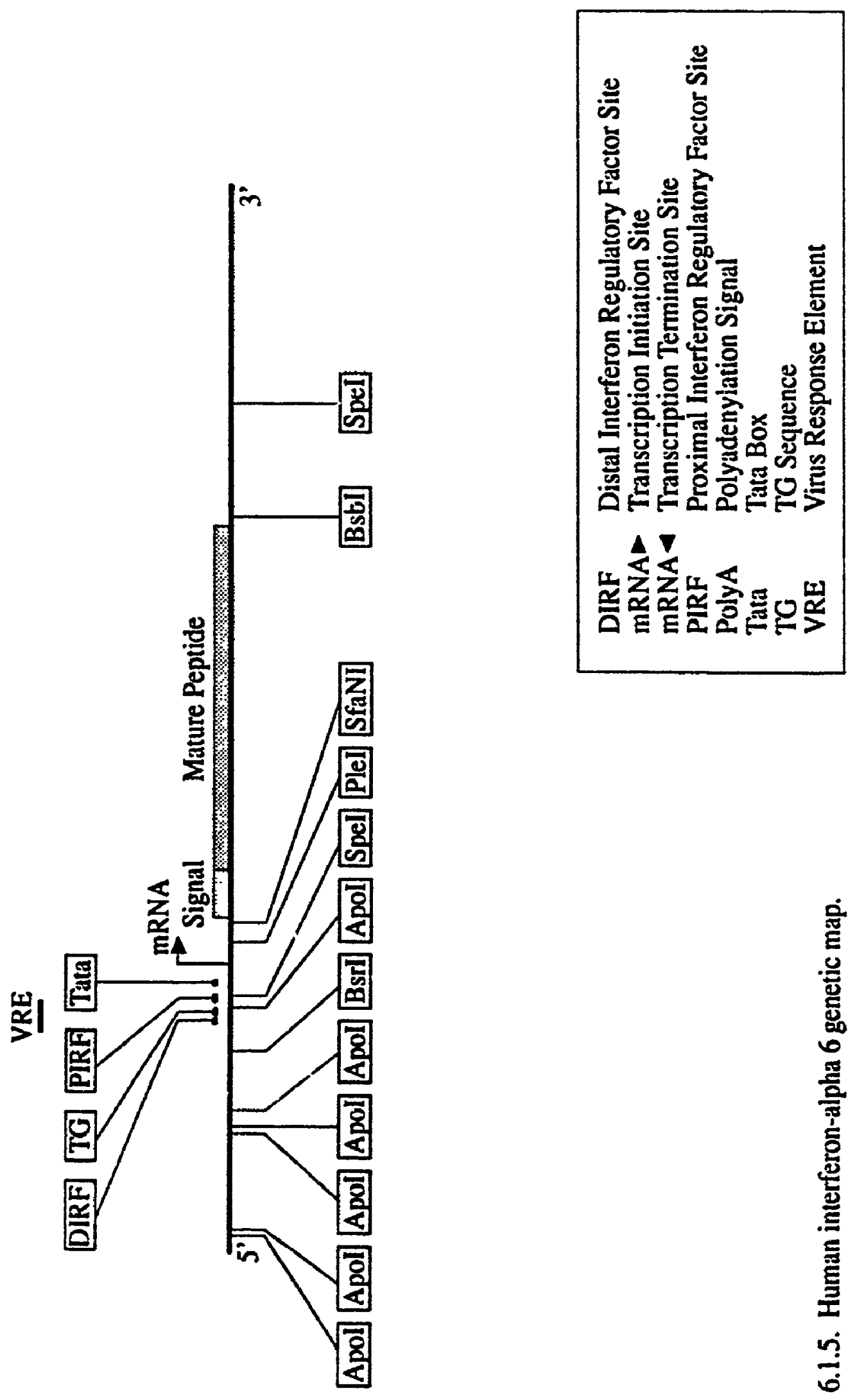

홓

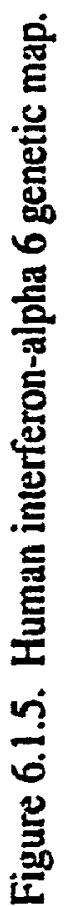



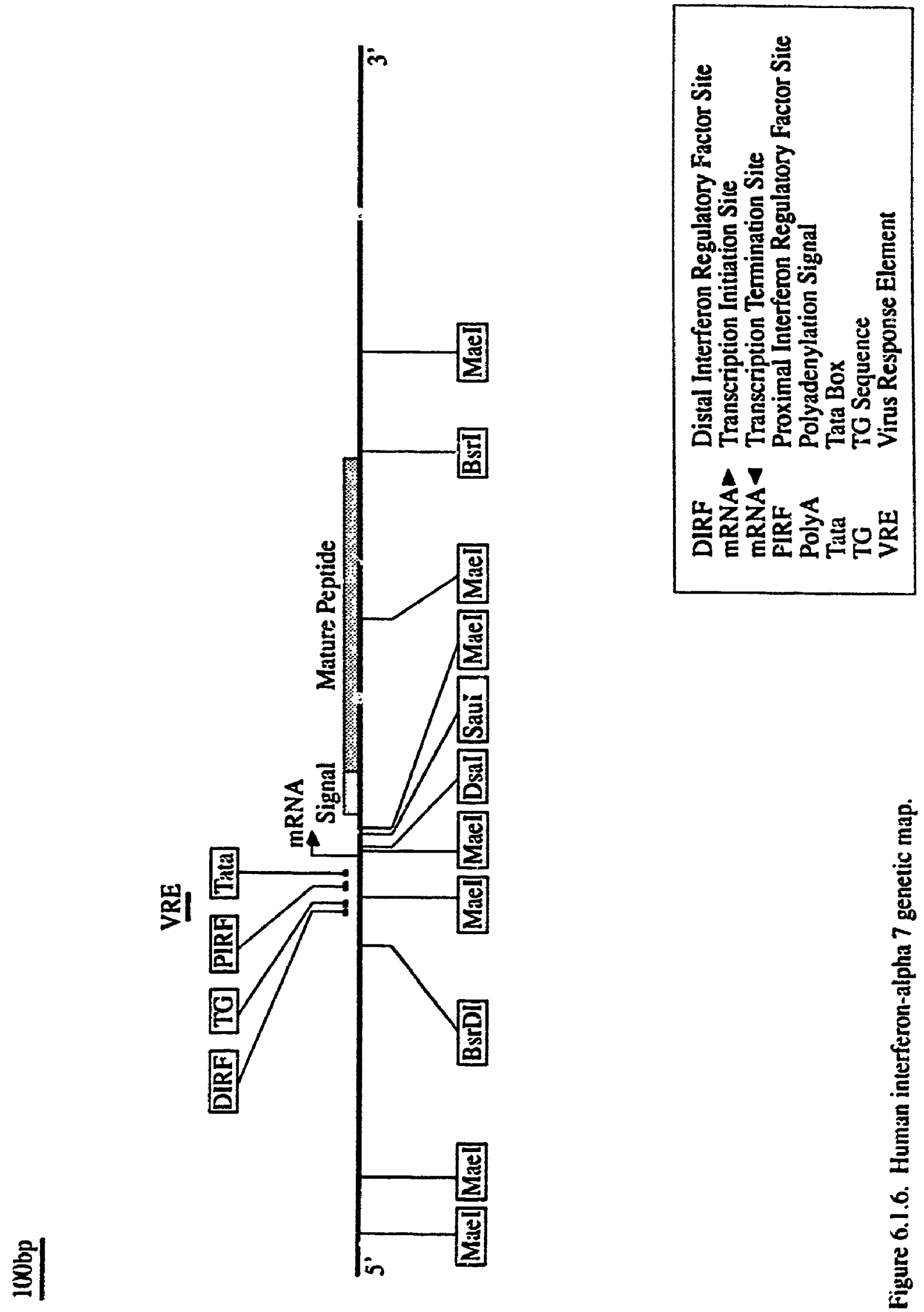

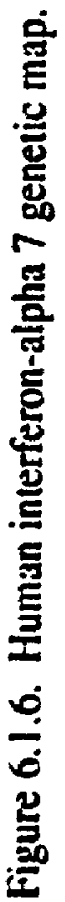



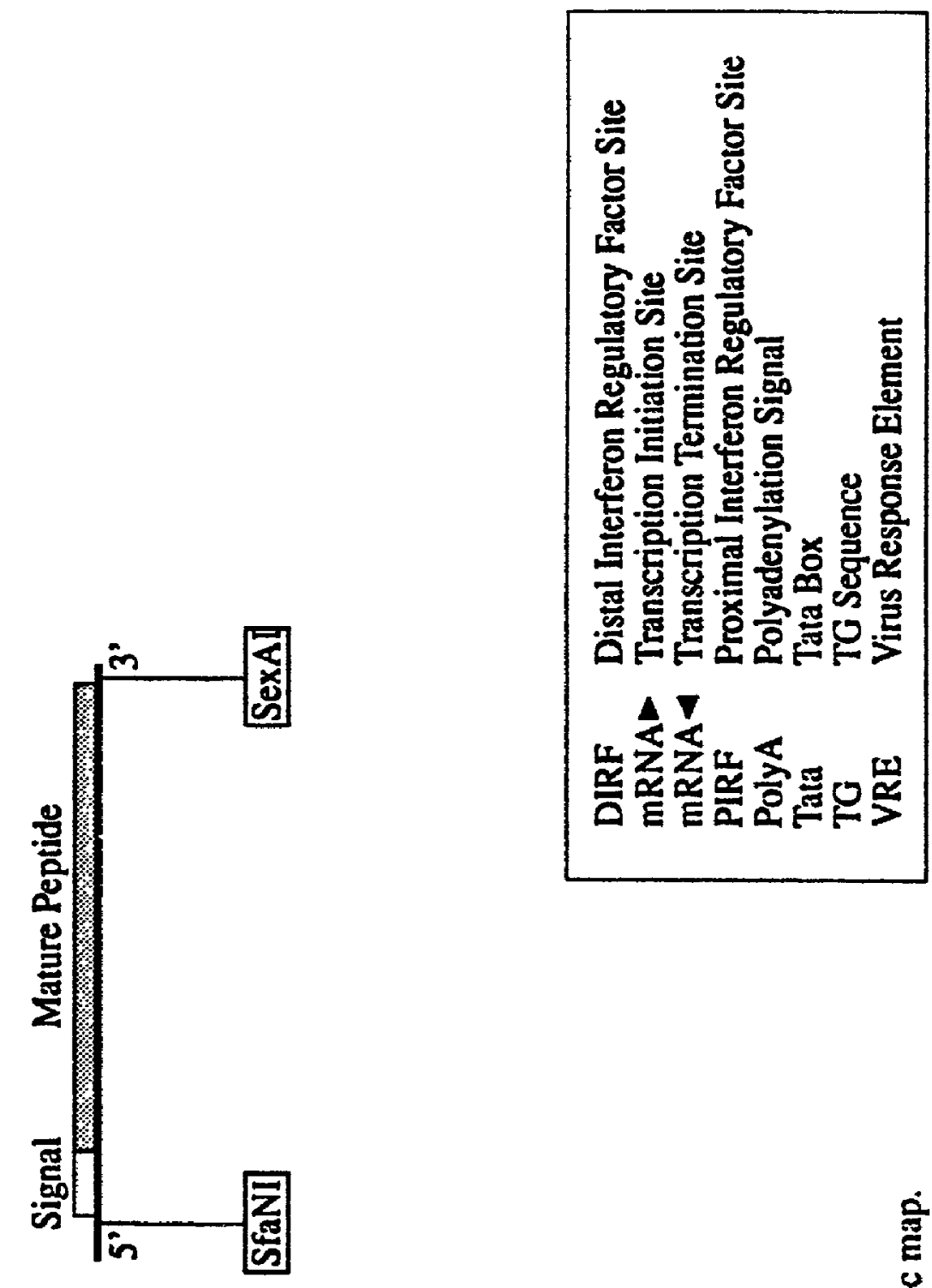

향

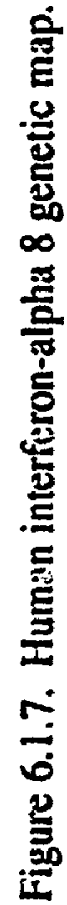



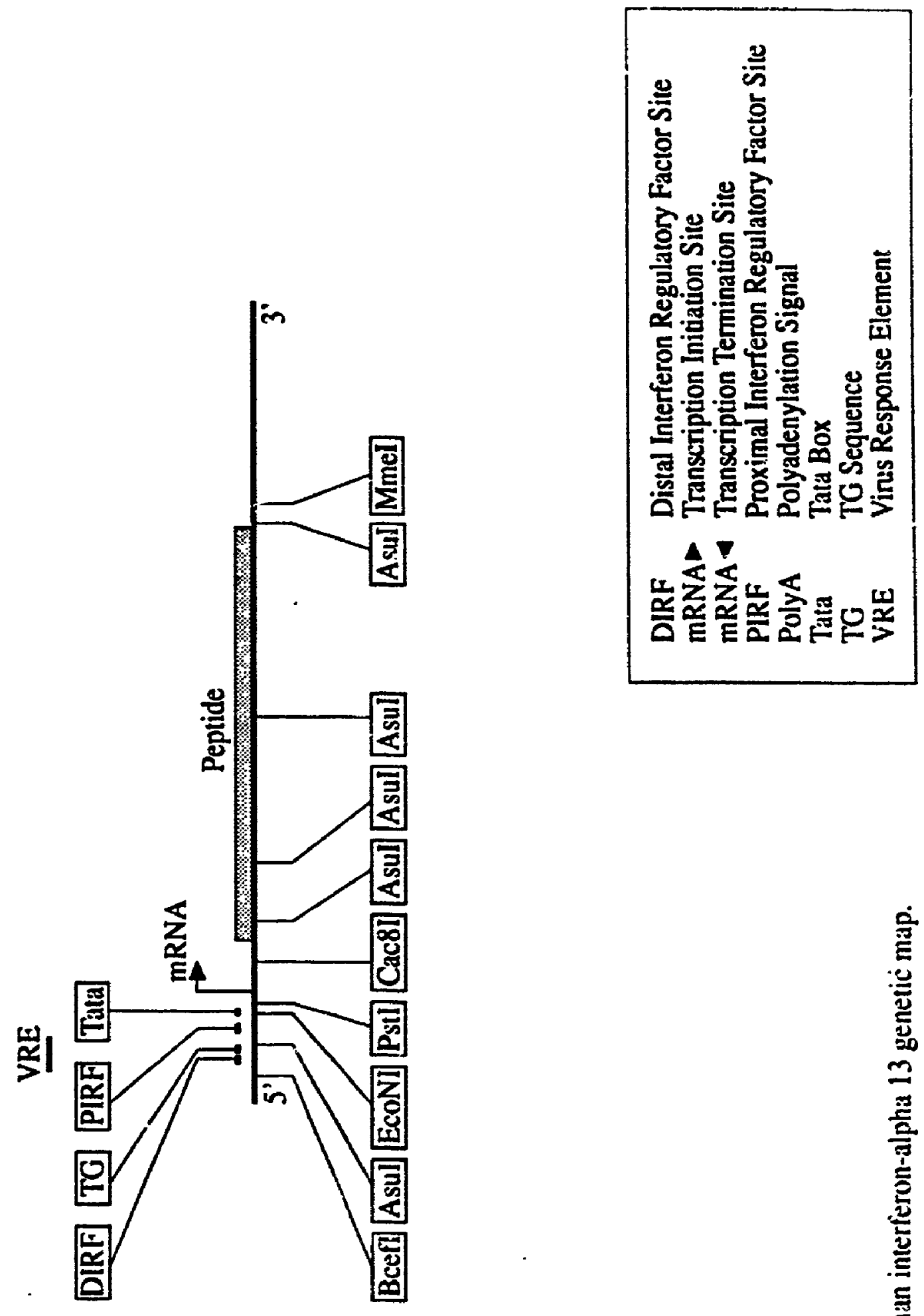

휭

是 

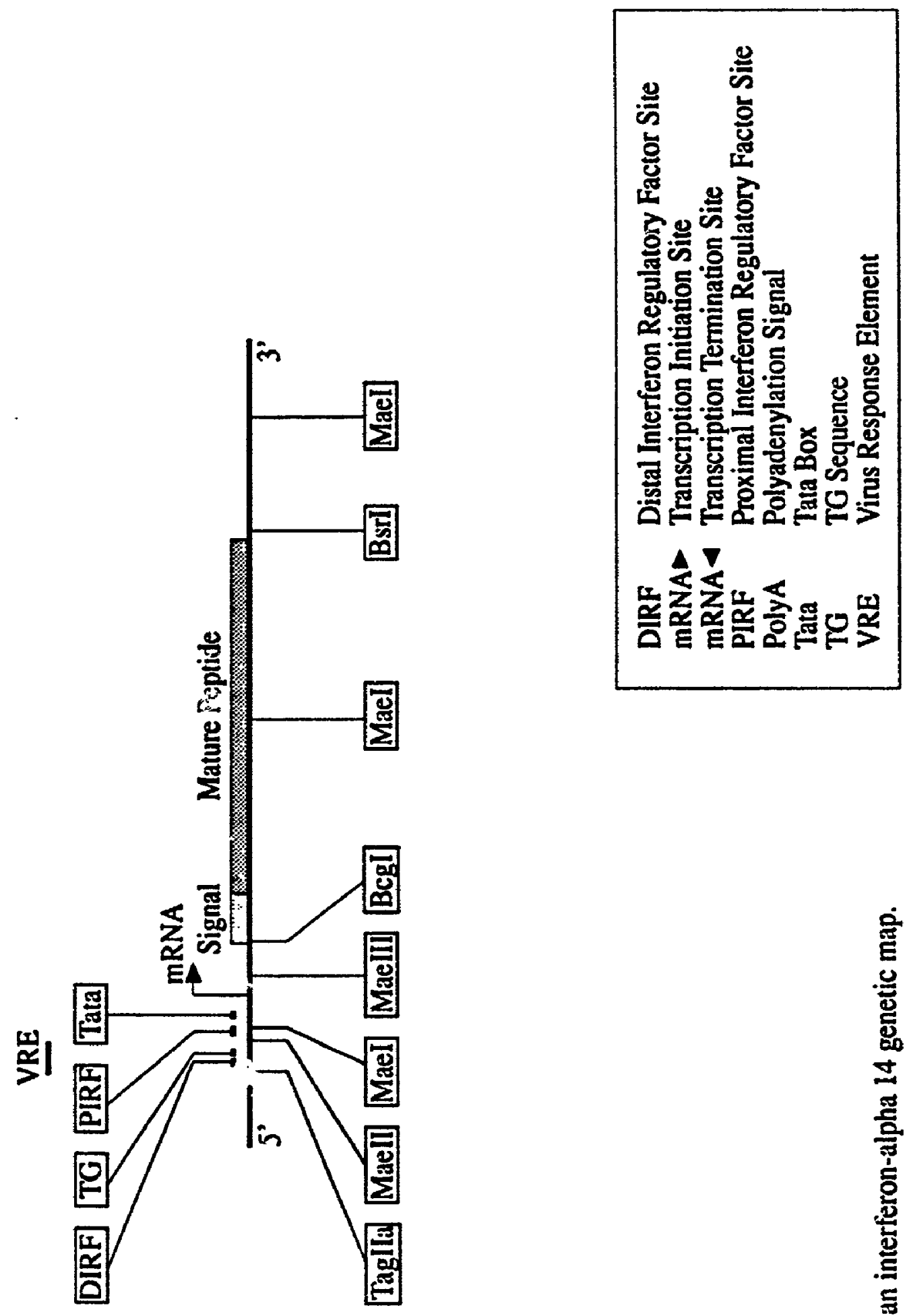

횧

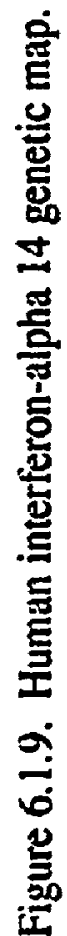




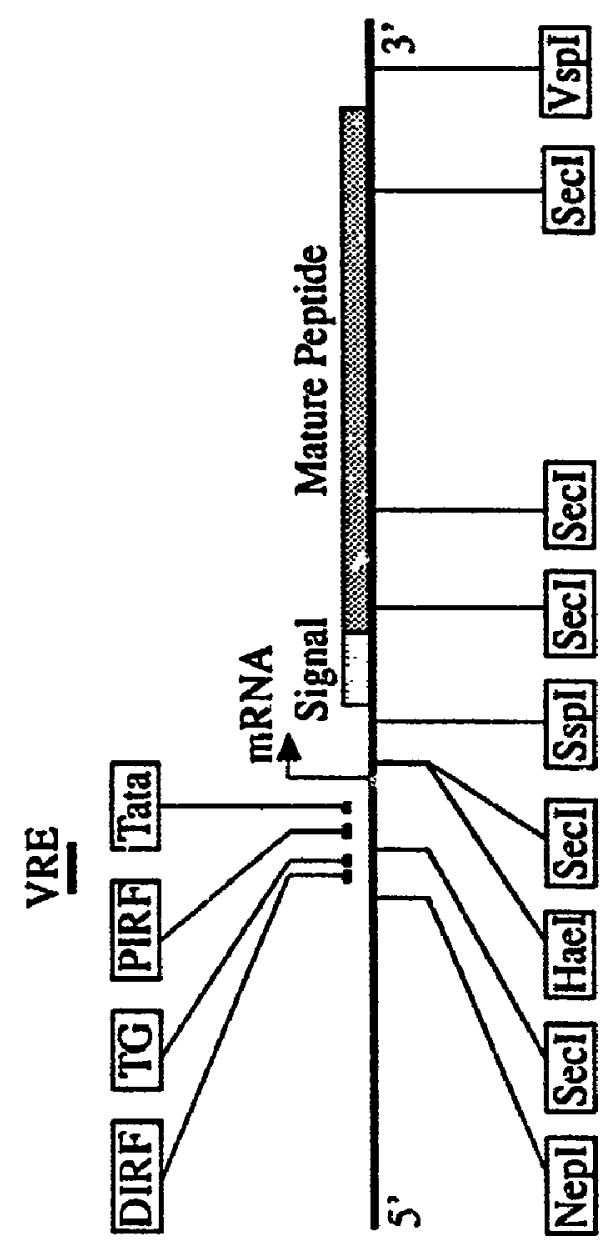

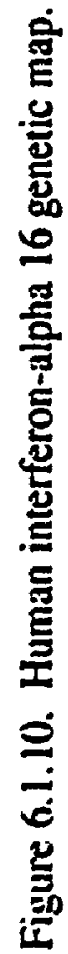



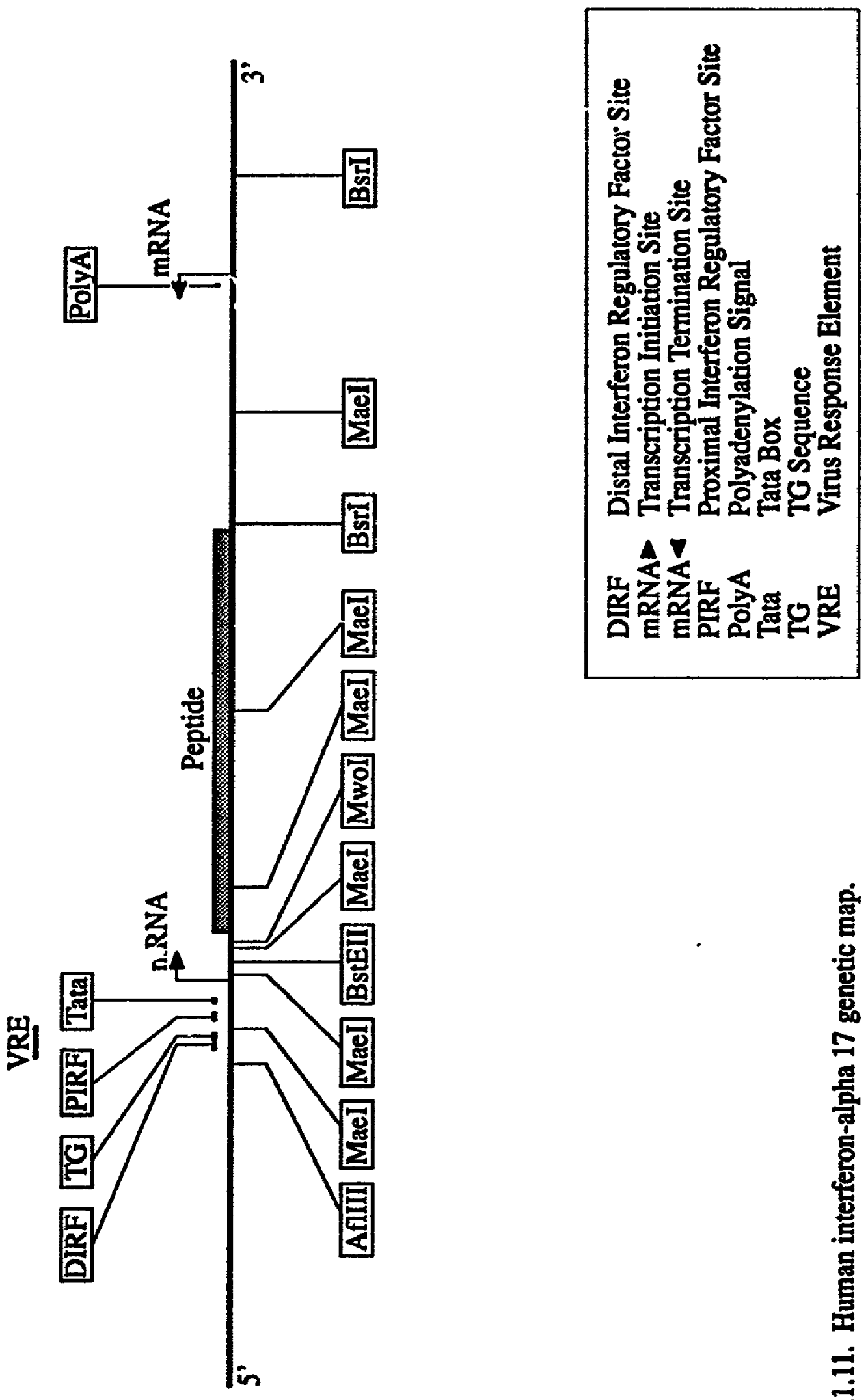

휘

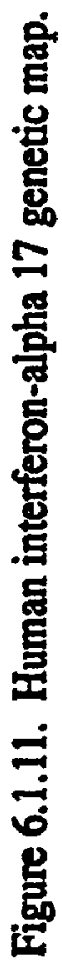




\subsection{Human Interferon-Alpha Promoter Transcription Maps}

Human interferon-alpha promoter transcription maps are restricted to the promoter regions. The human interferon-alpha promoter transeription maps indicate six types of promoter elements: 1. distal interferon regulatory factor site (DIRF), 2. proximal interferon regulatory factor site (PIRF), 3. tata box (Tata), 4. TG sequence (TG), 5. virus-response element (VRE) and 6. transcription initiation site (Cap). The diagrams further denote six multiple-sequence alignment regions: 1. RepA, 2. RepB, 3, R1, 4. R2, 5. R1' and 6. R2' (Ryals et. al., 1985). The human interferon-alpha promoter transcription maps are presented (Figures 6.2.1. - 6.2.10.).

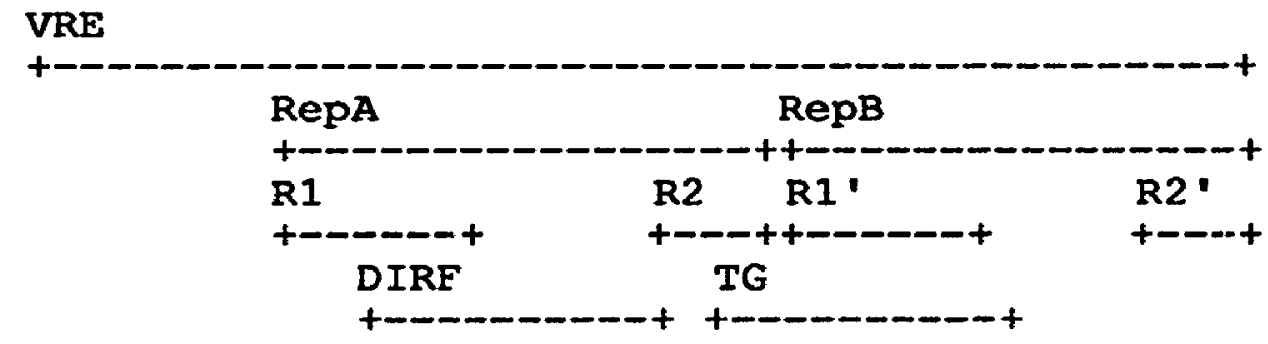

gagtgcatgaaggaaagcaaaaacagaaatggaaagtggcccagaagcattaa 1

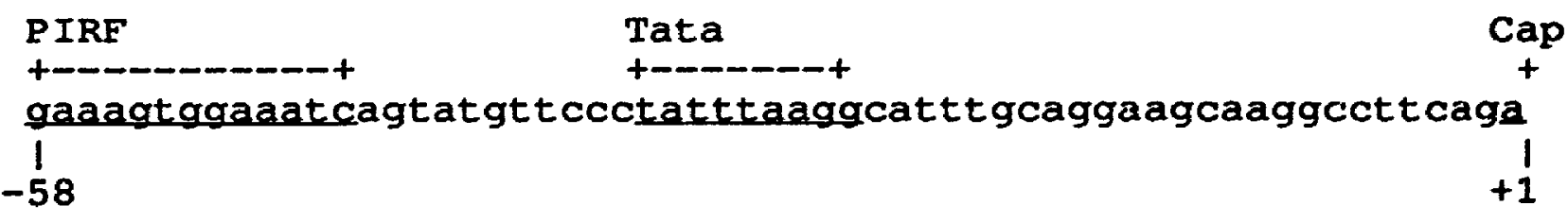

Figure 6.2.1. Human interferon-alpha 1 promoter transcription map. 

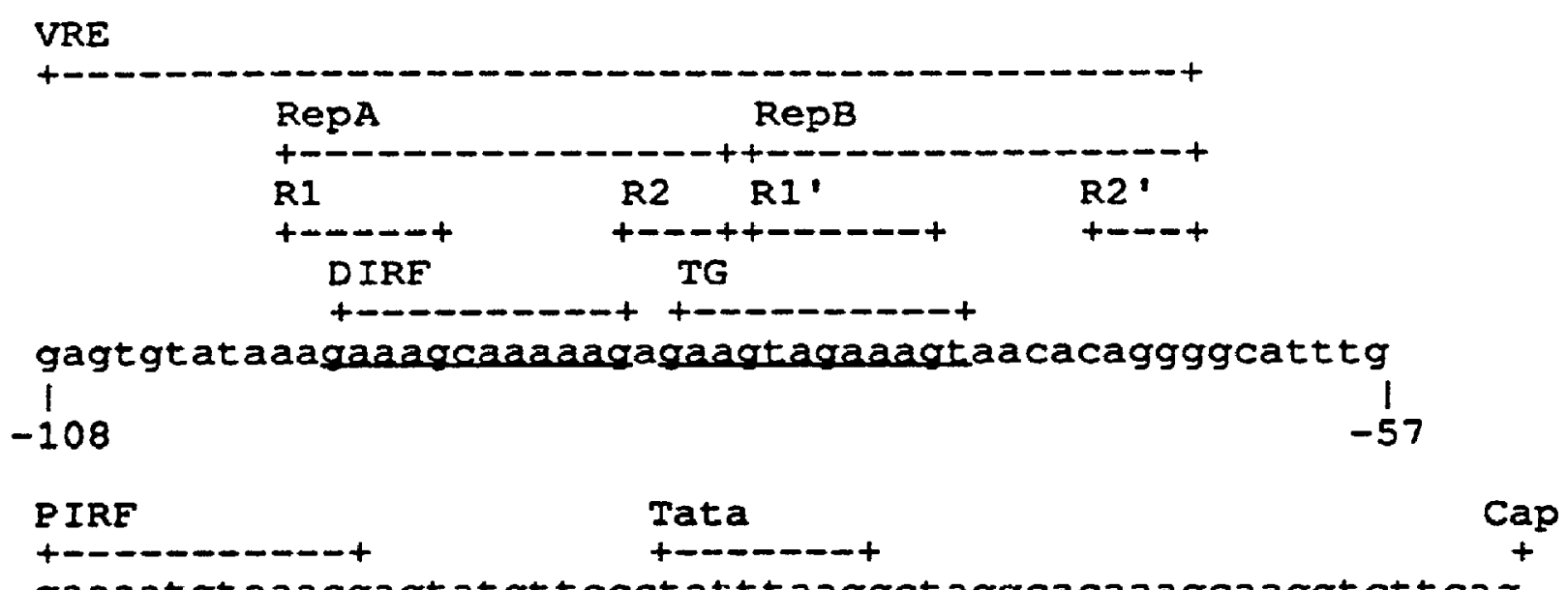

gaaaatotaaacgagtatgttccctatthaaggctaggcacaaagcaaggtcttcag 1

$-56$

Figure 6.2.2. Human interferon-alpha 2 promoter transcription map.

VRE

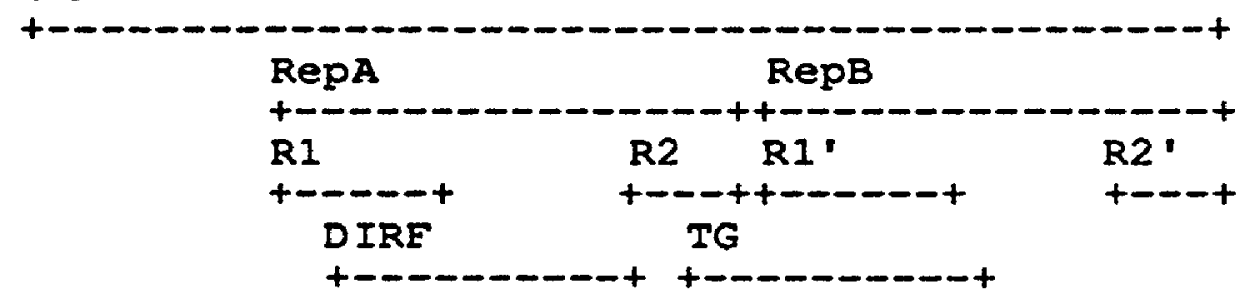

gagtgcgtaaagaaagcaaaaacagagatagaaagtacaactagggaattta I

$-108$

$-57$

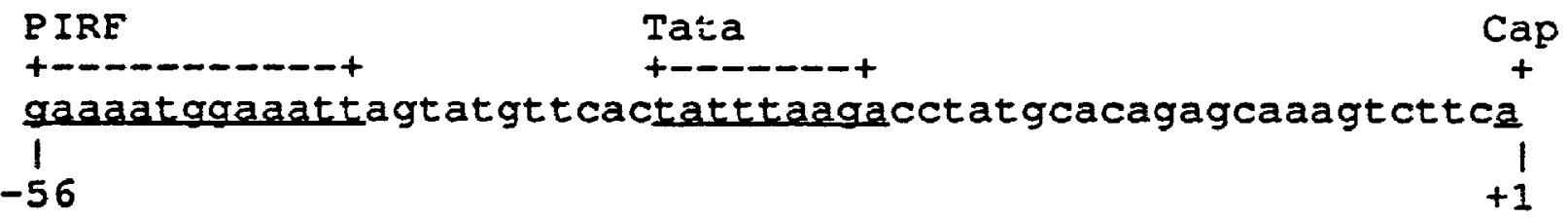

Figure 6.2.3. Human interferon-alpha 4 promoter transcription map. 


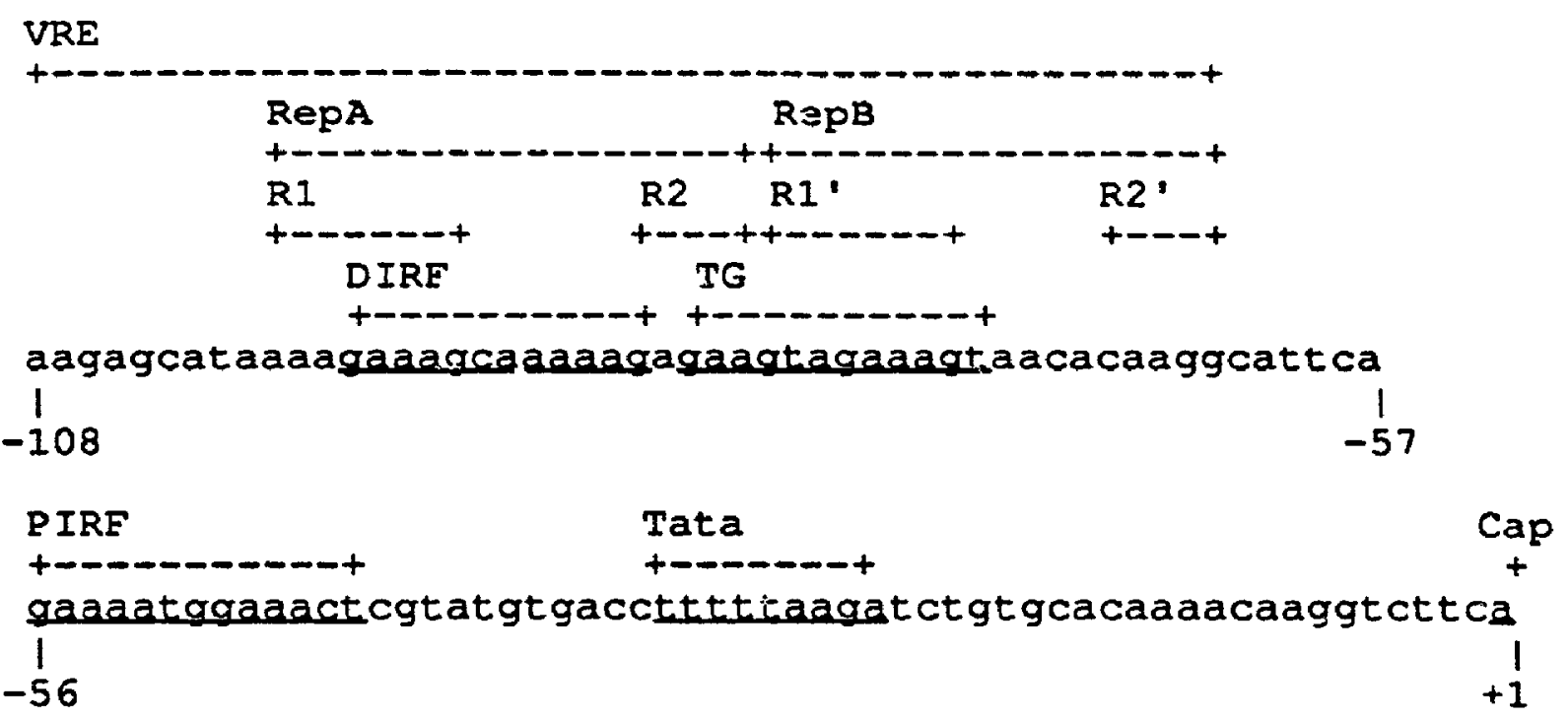

Figure 6.2.4. Human interferon-alpha 5 promoter transcription map.

VRE

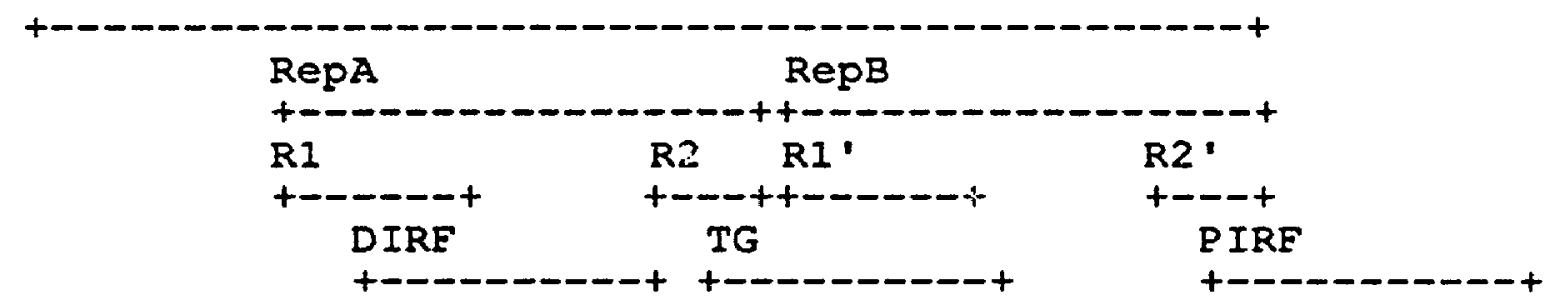

gagtaaataaatgaaagcaaatcagacgtagaaagtaaattctgaaaatggaaact 1

$-100$

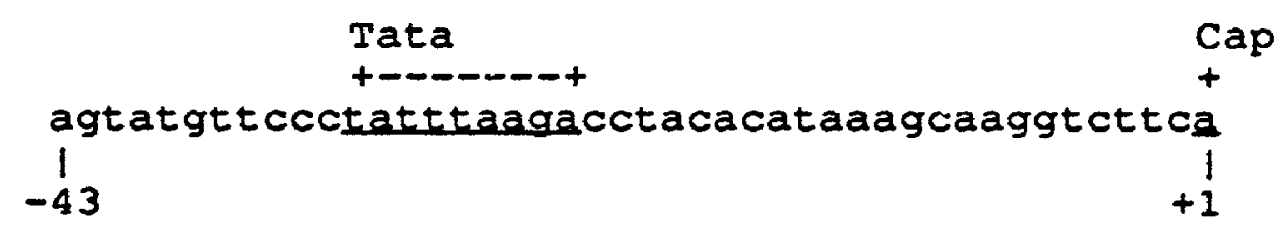

Figure 6.2.5. Human interferon-alpha 6 promoter transcription map. 
VRE

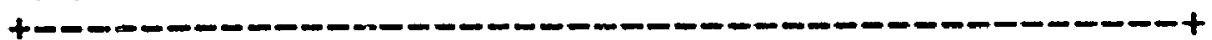

RepA RepB

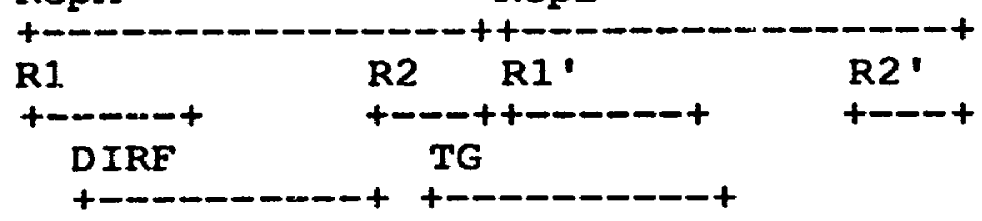

gagtgcataaagaaagcaaaaacagagatagaaagtaaaactagggcattta

1

$-108$

$-57$

$\begin{array}{llc}\text { PIRF } & \text { Tata } & \text { Cap } \\ +--+ & +- & +\end{array}$

gaaaatgraaattagtatgttcactatttaagacctatgcacagagcaaagtctcca 1

$-56$

Figure 6.2.6. Human interferon-alpha 7 promoter transcription map.

VRE

tre+che

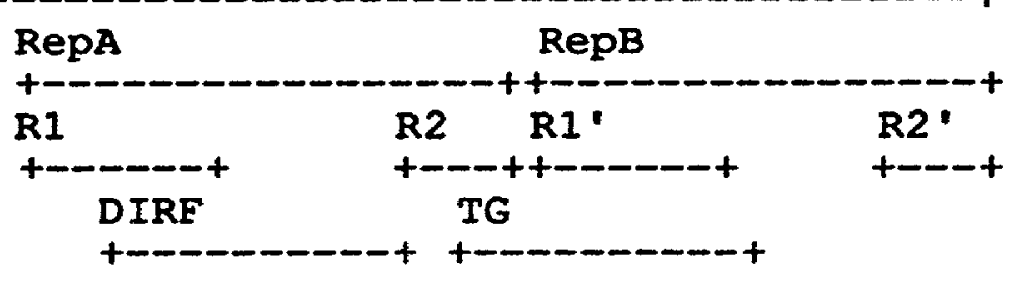

gagttcaagaaggaaagcaaaaacagaaatggaaagtggtccagaagcattaa 1

$-109$

Tata

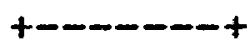

Cap

+---- - - - - +

gaaagtggaaatcagtatgttccctatttaaggcatctgcaggaagcaaagccttca

$-56$

Figure 6.2.7. Human interferon-alpha 13 promoter transcription map. 
VRE

$+-$

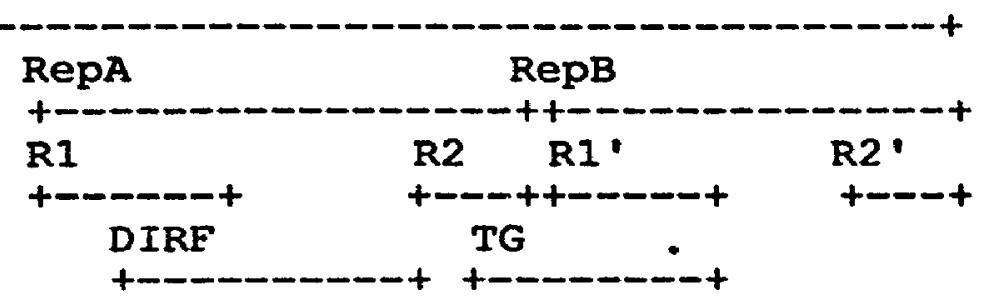

caggacataaaggaaagccaaaagagaagtagaaaaaacatgaagacgttca

1

$-109$

$-57$

PIRF 1 Tata + Cap

gaaaatggaagctagtatgttccttatttaagacctatgcacagagcaaggtcttca

1

$-56$

$+1$

Figure 6.2.8. Human interferon-alpha 14 promoter transcription map.

VRE

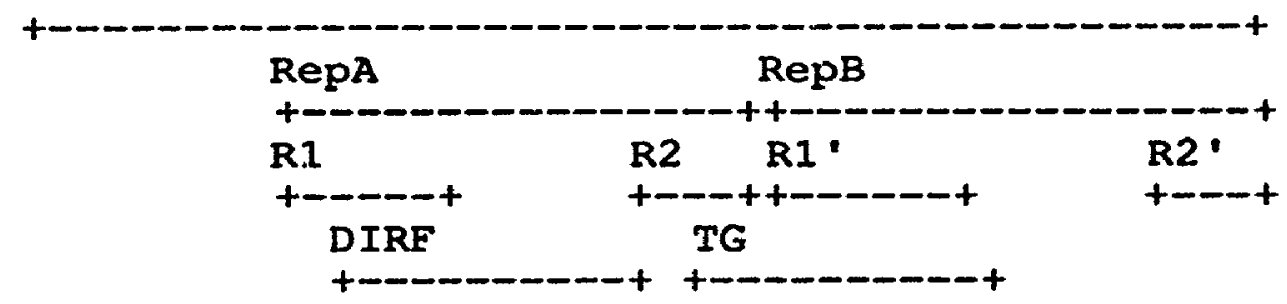

gagtgcataaagaaagcaaadacagagatagaaagtaaaaccagggcattta

1

$-108$

$-57$

$\begin{array}{lcc}\text { PIRF } & \text { Tata } & \text { Cap } \\ ++ & + \\ \text { gaaaatggaaattagtatgttcactatttaagaactatgcacagagcaagtcttca }\end{array}$ 1

$-56$

Figure 6.2.9. Human interferon-alpha 16 promoter transcription map. 


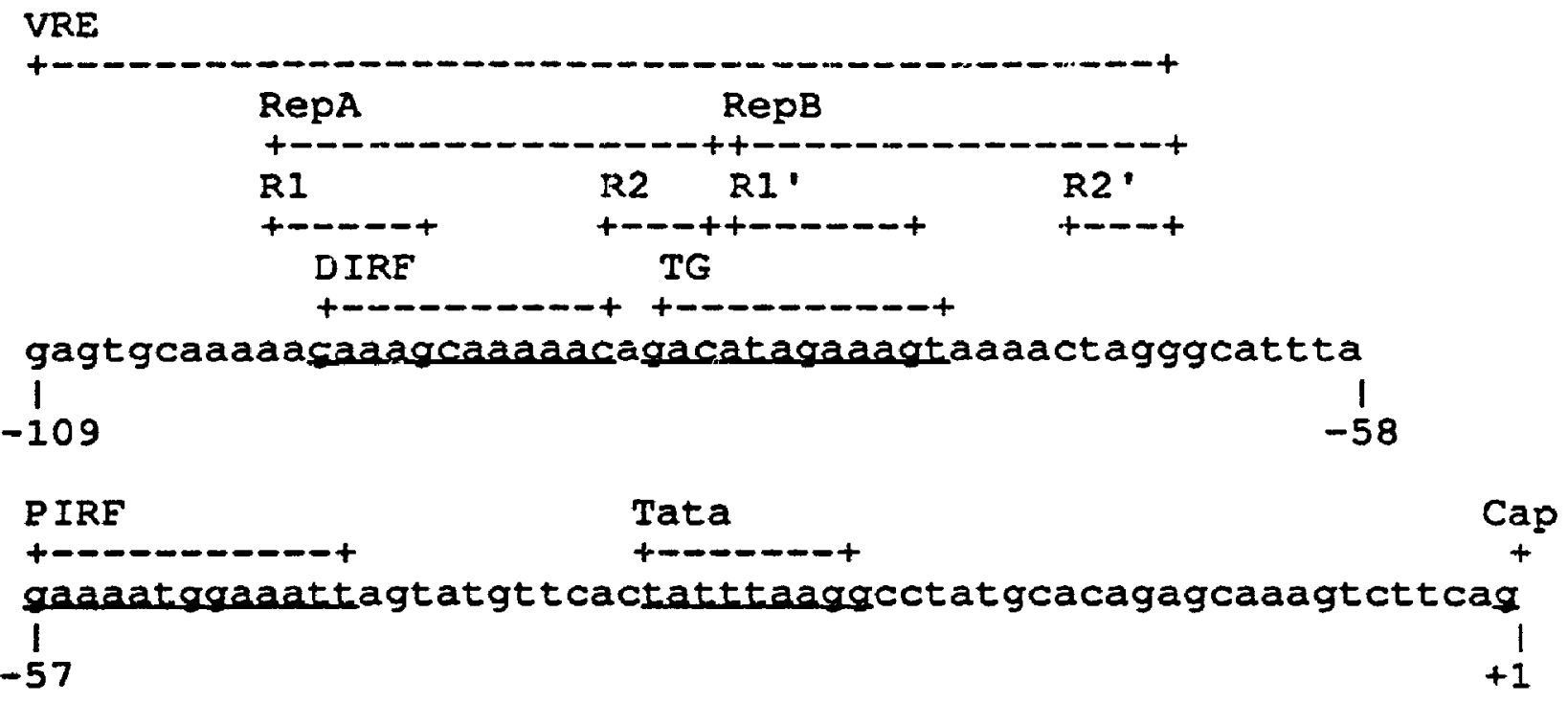

Figure 6.2.10. Human interferon-alpha 17 promoter transcription map. 


\subsection{Human Interferon-Alpha Promoter Elements}

Transcription analysis of the human interferon-alpha genes indicates a series of transcription control elements that occur within all human interferon-alpha promoters. Five potential human interferon-alpha promoter elements have been identified: 1 . tata boxes (Tata), 2. virus-response elements (VRE), 3. distal interferon regulatory factor (DIRF) sites, 4. proximal interferon regulatory factor (PIRF) sites and 5. TG sequences (TG). The human interferon-alpha promoter elements are presented (Figures 6.3.1. - 6.3.5.).

The tata box consensus sequence is twtttaagr. The tata boxes are 9 nucleotides long. The tata box 5 ' boundaries occur at positions -34 to -32 nucleotides relative to the cap site. The human interferon-alpha tata boxes have been previously discovered. Thus, the current tata box results are strictly confumatory. The tata boxes are presented (Figure

$$
\text { 6.3.1.). }
$$

Gene Sequence

\begin{tabular}{|c|c|}
\hline & Pata \\
\hline $6 F$ & tatttaagg \\
\hline IE & tatttaagg \\
\hline IENA4 & tatttaaga \\
\hline IENA5 & tttttaaga \\
\hline IENA 6 & tatttaaga \\
\hline IENA7 & tatttaaga \\
\hline IENA13 & tatttaagg \\
\hline IENA14 & tatttaaga \\
\hline IENA1 6 & tat $\pm t a a g a$ \\
\hline IFNA17 & atttaagg \\
\hline IFNA-CO & wetta \\
\hline
\end{tabular}

Figure 6.3.1. Tata boxes.

Ryals et. al. (1985) performed a multiple-sequence alignment on the virus-response elements. The virus-esponse elements of seven human interferon-alpha genes were aligned: IFNA1, IFNA2, IFNA4, IFNA5, IFNA7, IFNA13 and IFNA14. Six multiple- 
sequence alignment regions were defined: 1. RepA, 2. RepB, 3. R1, 4. R2, 5. R1' and 6. R2'. The multiple-sequence alignment was extended tc identify the virus-response elements of three additional human interferon-alpha genes: IFNA6, IFNA16 and IFNA17.

The virus-response elements include three transcription factor binding sites: 1. distal interferon regulatory factor site, 2. proximal interferon regulatory factor site and 3. TG sequence. Distal interferon regulatory factor sites and TG sequences are present within all virus-response elements. A proximal interferon regulatory factor site is present within the human interferon-alpha 6 virus-response element. The virus-response element consensus sequence is vagddhrwraa(d)gaaagcmaaawsagavrtrgaaa(g)(t)rvhh(y)cwdrr. The virus-response elements are 44 - 47 nucleotides long. The virus-response element $5^{\prime}$ boundaries occur at positions -111 to -100 nucleotides relative to the cap site. The virusrespnnse elements are presented (Figure 6.3.2.).

Gene Sequence

IFNA1

VRE

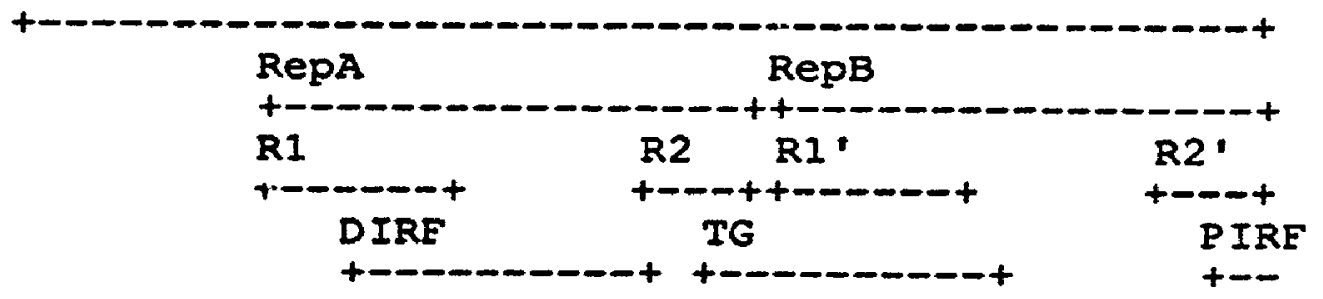

IFNA2

gagtgcatgaaggaaagcaaaaacagaaatggaaagtggce cagaa gagtgtataaa gaagcaaaaagagaagtagaaagtaaca caggg gagtgcgtaaa gaaagcaaaaacagagatagaaagtacaa ctagg aagagcataaagaaagcaaaaagagaagtagaaagtaaca caagg gagtaaataa tgaaagcaaaatcagacqtagaaagtaaat tctgaa gagtgcataaa gaaagcaaaaacagagatagaaagtaaaa ctagg gagttcaagaaggaaagcaaaaacagaaatgraaagtggtc cagaa caggacataaggraadccaaaagagaagtagaaa aaa catga gagtgcataa gaajocaaaacagagatagaaagtaaaccaggg gagtgcaaaa gaagcaaaacagacatagaaagtaaaa ctagg IFNA5 IFNA6 IFNA7 IFNA13 IFNA14 IFNA16 IENA17 IFNA-CONS vagddhrwraadgaaagcmaaawsagavetrgaaagtrvihycwdrr

Figure 6.3.2. Virus-response elements. 
Interferon regulatory factor recognition sequences have been investigated. Harada et. al. (1989) claim that the distinct transeription factors interferon regulatory factor 1 and interferon regulatory factor 2 bind to identical regulatory sequences. In binding site selection experiments, the interferon regulatory factor 1 and interferon regulatory factor 2 recognition sites were determined to be b(ajawa(n)y(r)amavn and s(a)waa(s)ygaaasb respectively (Tanaka et. al., 1993).

The presence of two interferon regulatory factor binding sites within the human interferon-alpha 1 promoter was first proposed by MacDonald et. al. (1990). Two potential interferon regulatory ractor binding sites were identified in all human interferonalpha promoters. On the basis of their relative $5^{\prime}$ distances from the transcription initiation site, the two sites are designated: 1. distal interferon regulatory factor site and 2. proximal interferon regulatory factor site.

The distal interferon regulatory factor site consensus sequence is gaaagcmaaaws. The distal interferon regulatory factor sites are 12 nucleotides long. The distal interferon regulatory factor site $5^{\prime}$ boundaries occur at positions -99 to -85 nucleotides relative to the cap site. The proximal interferon regulatory factor site consensus sequence is gaaartgkaaryb. The proximal interferon regulatory factor sites are 13 nucleotides long. The proximal interferon regulatory factor site 5' boundaries occur at positions -58 to--56 nucleotides relative to the cap site. The distal interferon regulatory factor sites and proximal interferon regulatory factor sites are presented (Figures 6.3.3. - 6.3.4.). 
Gene Sequence

$\begin{array}{ll} & \text { DIRE } \\ & +------+ \\ \text { IFNA1 } & \text { gaaagcaaaaac } \\ \text { IFNA2 } & \text { gaaagcaaaag } \\ \text { IFNA4 } & \text { gaaagcaaaac } \\ \text { IFNA5 } & \text { gaaagcaaaaag } \\ \text { IFNA6 } & \text { gaaagcaaaatc } \\ \text { IFNA7 } & \text { gaaagcaaaaaC } \\ \text { IFNA13 } & \text { gaaagcaaaaac } \\ \text { IFNA14 } & \text { gaaagccaaaag } \\ \text { IFNA16 } & \text { gaaagcaaaaac } \\ \text { IFNA17 } & \text { gaaagcaaaaac } \\ \text { IFNA-Cons } & \text { gaaagcmaaaws }\end{array}$

Figure 6.3.3. Distal interferon regulatory factor sites.

$\begin{array}{ll}\text { Gene } & \text { Sequence } \\ & \text { PIRF } \\ & \text { t---------t } \\ \text { gaaagtggaaatc } \\ \text { IFNA1 } & \text { gaaaatgtaaacg } \\ \text { IFNA2 } & \text { gaaaatggaaatt } \\ \text { IFNA4 } & \text { gaaaatggaaact } \\ \text { IFNA5 } & \text { gaaaatggaaact } \\ \text { IFNA6 } & \text { gaaaatggaaatt } \\ \text { IFNA7 } & \text { gaaagtggaaatc } \\ \text { IFNA13 } & \text { gaaaatggaagct } \\ \text { IFNA14 } & \text { gaaaatggaaatt } \\ \text { IFNA16 } & \text { gaaaatggaaatt } \\ \text { IFNA17 } & \text { gaaartgkaaryb }\end{array}$

Figure 6.3.4. Proximal interferon regulatory factor sites.

The TG factor is a transcription factor active in the transcriptional activation of the human interferon-alpha 1 gene. The human interferon-alpha 1 TG sequence was determined to be gaaatggaaagt (MacDonald et. al., 1990). Potential TG sequences were identified in the remaining human interferon-alpha promoters. The TG sequences are located within the virus-response elements. Observe that the human interferon-alpha 13 TG sequence gaaatggaaagt is identical to the human interferon-alpha 1 TG sequence. These findings suggest the existence of a potential family of TG sequences. The TG consensus sequence is gavrtrgaaa $(g)(t)$. The TG sequences are $10-12$ nucleotides long. The TG 
sequence 5 boundaries occur at positions -86 to -75 nucleotides relative to the cap site. The TG sequences are presented (Figure 6.3.5.).

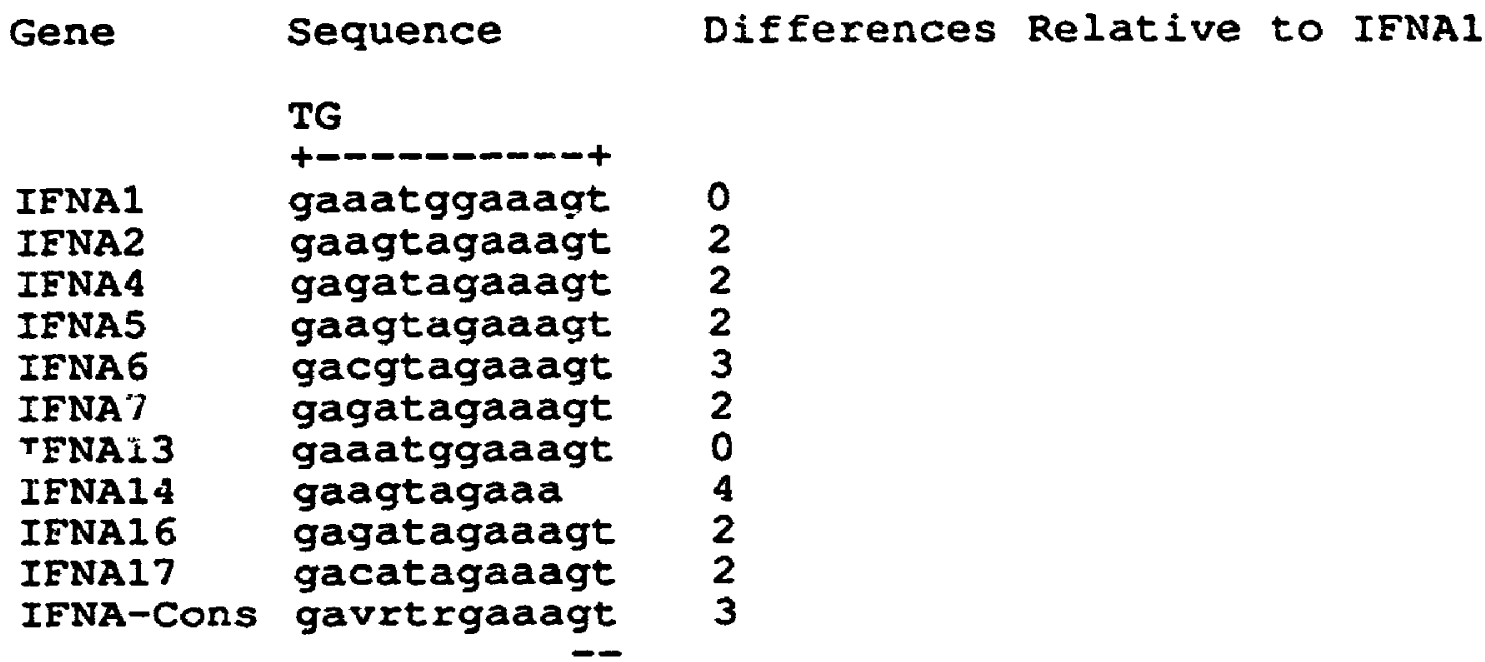

Figure 6.3.5. TG sequences.

The promoter element consensus sequences are summarized (Table 6.3.1.).

Name Sequence

Tata twtttaagr

VRE vagddhrwraa(d)gaaagcmaaawsagavrtrgaaa(g)(t)rvhh(y)cwdrr

DIRF gaaagcmaaaws

PIRF gaaartgkaaryb

TG gavrtrgaaa $(g)(t)$

Table 6.3.1. Promoter element consensus sequences. 


\subsection{Human Interferon-Alpha Transcription Regulatory Mechanisin}

The human interferon-alpha genes are activated by viral infection. The human interferon-alpha gene regulatory region extends -111 to $\cdot 100$ nucleotides 5 ' relative to the transcription initiation site. The human interferon alpha gene regulatory iegion contains four promoter elements responsible for the transcriptional regulation of viral induction: 1 . tata box (Tata), 2. regulatory domain 1 (RD1), 3. regulatory domain 2 (RD2) and 4. regulatory domain 3 (RD3).

The tata box is the binding sequence recognized by transcription factor TFIID. Transcription factor TFIID is responsible for positioning RNA polymerase II at the transcription initiation site. The tata box consensus sequence is twttraagr. Tata boxes are 9 nucleotides long. Tata box $5^{\prime}$ boundaries occur at positions -34 to -32 nucleotides relative to the transcription initiation site.

Regulatory domain 1 corresponds to the proximal interferon regulatory factor site. Regulatory domain 1 could be recognized by either interferon regulatory factor 1 or interferon regulatory factor 2 . Interferon regulatory factor 1 is a transcriptional activetor whereas interferon regulatory factor 2 is a transcriptional repressor (Harada et. al., 1989). The regulatory domain 1 consensus sequence is gaaartgkaaryb. Regulatory domain 1 sequences are 13 nucleotides long. Regulatory domain 1 sequence $5^{\circ}$ boundaries occur at positions -58 to -56 relative to the cap site.

Regulatory domain 2 corresponds to the TG sequence. Regulatory domain 2 is recognized by the TG factor. The TG factor is a transcriptional activator of human interferon-alpha 1 (MacDonald et. al., 1990). The regulatory domain 2 consensus sequence is gavrtrgaaa $(g)(t)$. Regulatory domain 2 sequences are $10-12$ nucleotides long. Regulatory domain 2 sequence $5^{\circ}$ boundaries occur at positions -86 to -75 relative to the cap site. 
Regulatory domain 3 corresponds to the distal interferon regulatory factor site. Regulatory domain 3 could be recognized by either interferon regulatory factor 1 or interferon regulatory factor 2 . Interferon regulatory factor 1 is a transcriptional activator whereas interferon regulatory factor 2 is a transcriptional repressor (Harada et. al., 1989). The regulatory domain 3 consensus sequence is gaaagcmaaaws. Regulatory domain 3 sequences are 12 nucleotides long. Regulatory domain 3 sequence 5 boundaries occur at positions -99 to -85 relative to the cap site.

The human interferon-alpha transciption regulatory sequences are summarized (Table 6.4.1.) (Figure 6.4.1.).

\begin{tabular}{|c|c|c|}
\hline Regulatory Sequence & Transcription Factor & Function \\
\hline $\begin{array}{l}\text { tata box } \\
\text { regulatory domain } 1 \\
\text { regulatory domain } 2 \\
\text { regulatory domain } 3\end{array}$ & $\begin{array}{l}\text { TFIID } \\
\text { interferon regulatory factor } 1 \\
\text { interferon regulatory factor } 2 \\
\text { TG factor } \\
\text { interferon regulatory factor } 1 \\
\text { interferon regulatory factor } 2\end{array}$ & $\begin{array}{l}\text { RNA polymerase II orientation } \\
\text { transcriptional activation } \\
\text { transcriptional repression } \\
\text { transcriptional activation } \\
\text { transcriptional activation } \\
\text { transcriptional repression }\end{array}$ \\
\hline
\end{tabular}

Table 6.4.1. Human interferon-alpha transciption regulatory sequences. 

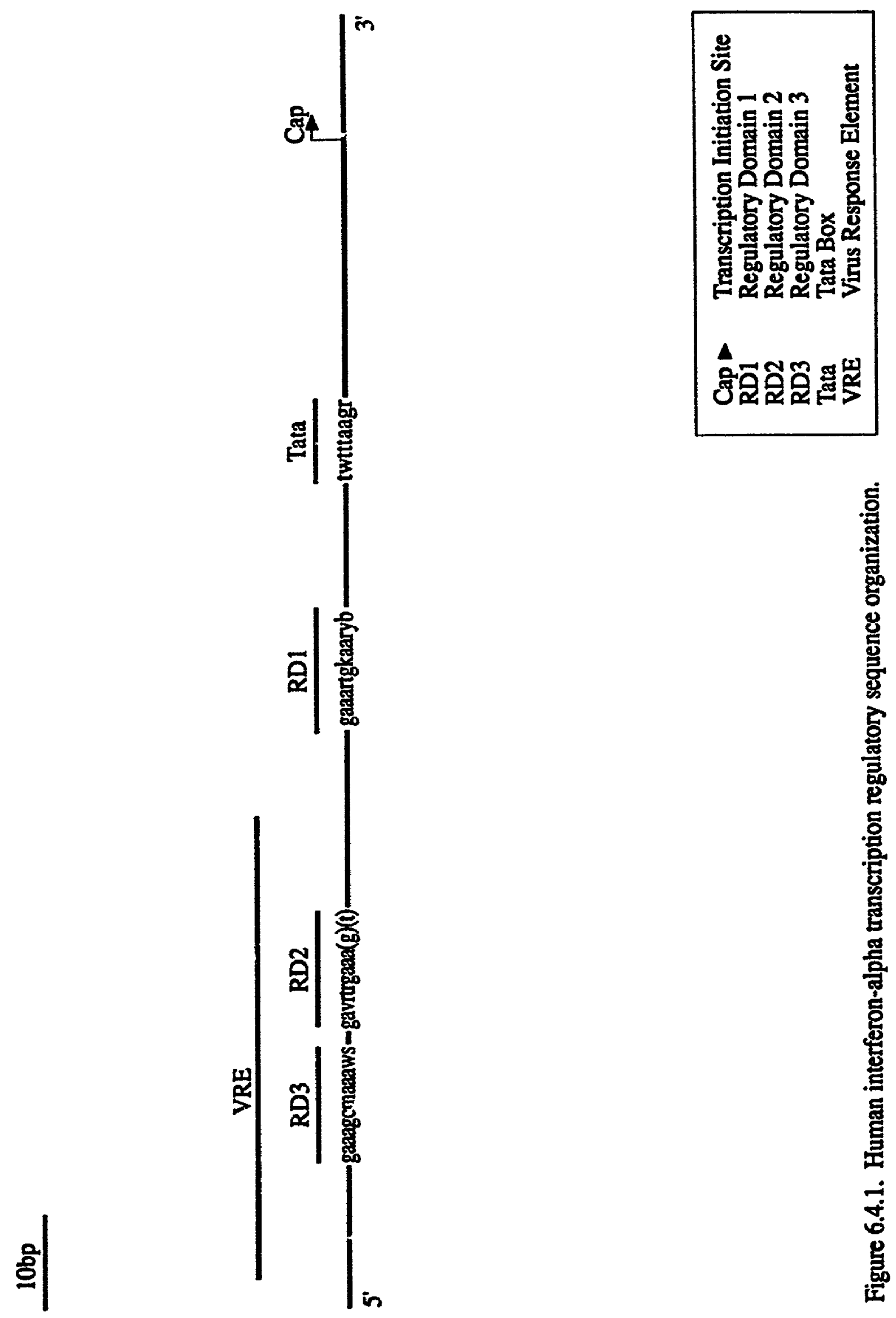
A theoretical model for the transcriptional regulation of human interferon-alpha is hypothesized. The virus-response element is critical to viral-induced transcription and is involved in transcriptional activation. Three functional components have been defined: regulatory domain 1, regulatory domain 2 and regulatory domain 3.

Regulatory domain 1 is located $3^{\prime}$ relative to the virus-response element. Regulatory domain 1 is recognized by interferon regulatory factor 2 . Regulatory domain 1 is reponsible for transcriptional repression and is renamed negative regulatory domain 1 (NRD1).

Regulatory domain 2 is located within the virus-response element. Regulatory domain 2 is recognized by the TG factor (MacDonald et. al., 1990). Regulatory domain 2 is responsible for transcriptional activation and is renamed positive regulatory domain 1 (PRD1).

Regulatory domain 3 is located within the virus-response element. Regulatory domain 3 is recognized by interferon regulatory factor 1 (MacDonald et. al., 1990). Regulatory domain 3 is responsible for transcriptional activation and is renamed positive regulatory domain 2 (PRD2).

The theoretical model of tuman interferon-alpha transcriptional regulation operates as follows. Interferon regulatory factor 2 normally binds to negative regulatory domain 1 . The repressor blocks transcription factor binding to positive regulatory domains 1 and 2 . Induction involves repressor inactivation. Interferon regulatory factor 1 and TG factor could then bind to positive regulatory domains 1 and 2 respectively. Transcription is then activated. This model is supported solely by computational evidence and requires experimental verification. The prototype model will require modification as additional transcription factor binding sites are discovered. 


\section{Chapter 7}

\section{Parallel Performance Analysis}

Three metrics for parallel perturmance analysis are defined: 1 . scaled speedup, 2 . cost and 3. scaled efficiency. Parallel performance of the nucleotide motif search algorithm was evaluated by nucleotide motif searches of the retinoblastoma gene. Parallel perfomance experiments used three sets of nucleotide motifs: 1. Restriction Enzyme Database (REBASE) release 405, 2. Transcription Factors Database (TFD) and 3. Human Interferon Transcription Factors Database (HuIFNTFD). All three parallel performance experiments demonstrated near-linear speedup curves. Parallel algorithm performance properties are further discussed.

\subsection{Performance Measures}

The term metric refers to a criterion used to measure the performance of a system. Parallel algorithms have two performance goals: 1 . minimize the execution time and 2 . equalize workload distribution across the processors. Parallel algorithm performance can be evaluated using three metrics: 1 . scaled speedup, 2 . cost and 3. scaled efficiency.

The scaled speedup of a parallel algorithm is the ratio between the parallel algorithm execution time requirements using a single processor on the parallel computer to the parallel algorithm execution time requirements using multiple processors on the parallel computer (Gustafson et. al., 1988). This ratio can exaggerate the actual speedup because it masks the parallel algorithm overhead. Therefore, the alternate term parailelizability is introduced to refer to the ratio between the time taken by a parallel algorithm executing on one processor to the time taken by the parallel algorithm executing on multiple processors. 
The cost of a parallel algorithm is a measure of the total number of operations performed. The cost of a parallel algorithm is defined as the product of the time elapsed and the number of processors used.

The scaled efficiency of a parallel algorithm is the scaled speedup divided by the number of processors used.

The parallel performance analysis involved nucleotide motif searches of the retinoblastoma gene. Three sets of nucleotide motifs were used: 1. Restriction Enzyme Database (REBASE) release 405, 2. Transcription Factors Database (TFD) and 3. Human Interferon Transcription Factors Database (HuIFNTFD). The retinoblastoma gene spans 180,388 nucleotides of genomic DNA (Toguchida et. al., 1993). In the current parallel performance analysis, the problem size (i.e. the number of retinoblastoma nucleotides) remained fixed as the number of processors was increased.

The parallel performance experiments are summarized (Table 7.1.1.). Parallel performance results are plotted as three types of graphs: 1 . speedup curves, 2 . cost curves and 3. efficiency curves. The parallel performance graphs are summarized (Table 7.1.2.).

Patterns

restriction endonucleases transcription factors human interferon transcription factors
Table

7.2.1.

7.3.1.

7.4.1.

Table 7.1.1. Parallel performance analysis experiments.

Patterns

restriction endonucleases restriction endonucleases restriction endonucleases transcription factors transcription factors transcription factors human interferon transcription factors human interferon transcription factors human interferon transcription factors
Curve Figure

$\begin{array}{ll}\text { speedup } & 7.2 .1 \\ \text { cost } & 7.2 .2 . \\ \text { efficiency } & 7.2 .3 \\ \text { speedup } & 7.3 .1 \\ \text { cost } & 7.3 .2 . \\ \text { efficiency } & 7.3 .3 \\ \text { speedup } & 7.4 .1 \\ \text { cost } & 7.2 .2 \\ \text { efficiency } & 7.4 .3 .\end{array}$

Table 7.1.2. Parallel performance analysis graphs. 


\subsection{Restriction Endonucleases}

The Restriction Enzyme Database (REBASE) is a comprehensive list of known restriction endonucleases (Roberts and Macelis, 1994). REBASE release 405 represents all known type II restriction endonucleases reported as of May 1, 1994. The retinoblastoma gene spans 180,388 nucleotides of genomic DNA (Toguchida et. al., 1993). The first parallel performance experiment involved a restriction analysis of the retinoblastoma gene using the REBASE release 405 type II restriction endonucleases. The retinoblastoma gene contained 24,699 type II restriction endonuclease recognition sites. The parallel performance results are presented (Table 7.2.1.). The speedup curve (Figure 7.2.1.), cost curve (Figure 7.2.2.) and efficiency curve (Figure 7.2.3.) are further presented.

$\begin{array}{rrrrr}\text { processors } & \begin{array}{r}\text { time } \\ \text { (seconds) }\end{array} & \text { speedup } & \text { cost efficiency } \\ 1 & 25.576360 & 1.000000 & 25.576360 & 1.000000 \\ 2 & 12.789119 & 1.999853 & 25.578238 & 0.999927 \\ 4 & 6.395067 & 3.999389 & 25.580268 & 0.999847 \\ 8 & 3.198284 & 7.996901 & 25.586272 & 0.999613\end{array}$

Table 7.2.1. Parallel performance analysis of restriction endonucleas $\dot{z}$ patterns. 


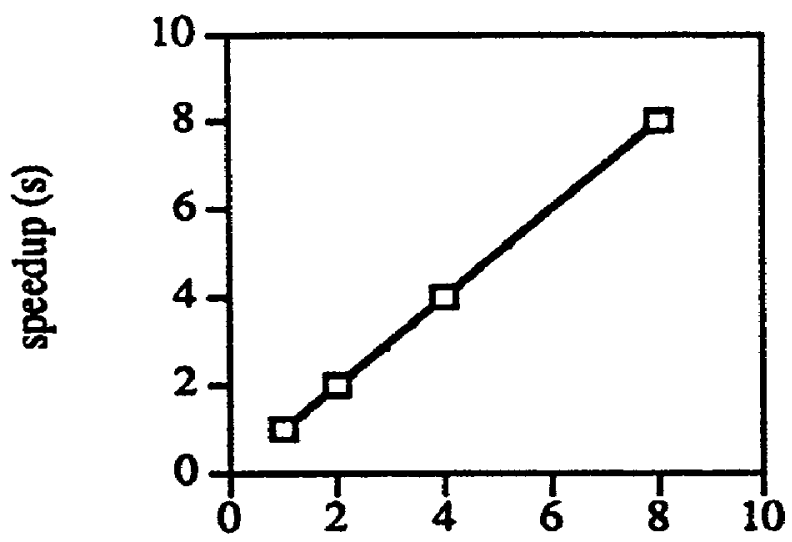

processors (p)

Figure 7.2.1. Speedup curve of restriction endonuclease pattems. The ideal speedup curve $s=p$ is plotted as a dashed line for comparison.

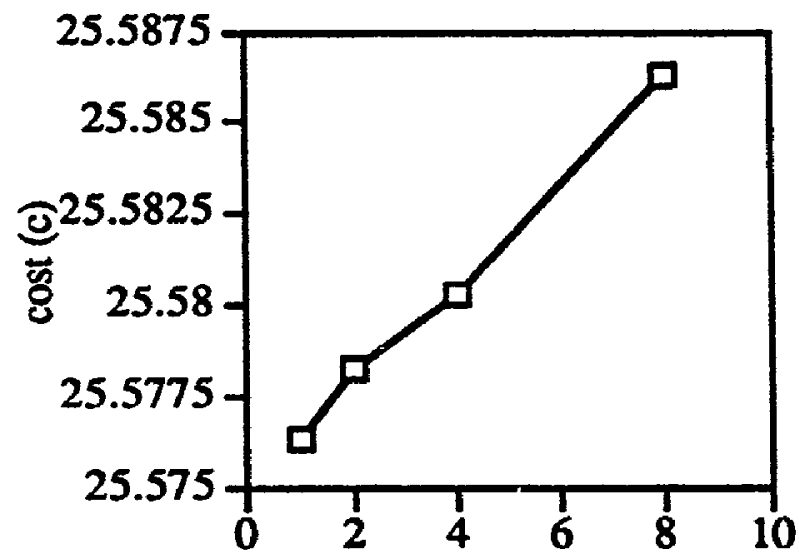

processors (p)

Figure 7.2.2. Cost curve of restriction endonuclease patterns. 


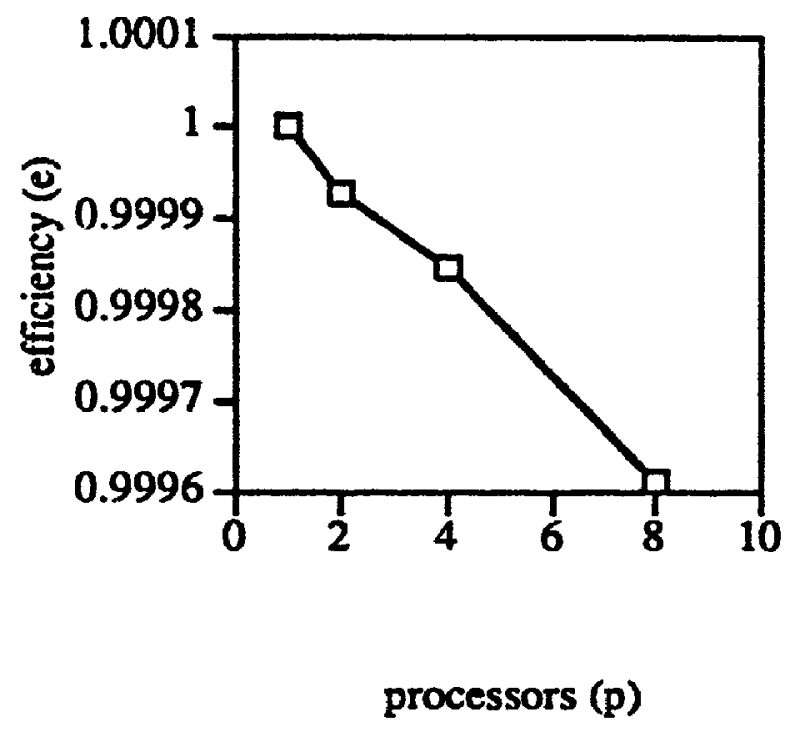

Figure 7.2.3. Efficiency curve of restriction endonuclease patterns. 


\subsection{Transcription Factors}

The Transcription Factors Database (TFD) is the largest compiled list of transcription factor recognition sequences (Ghosh, 1993). TFD represents transcription factors reported as of September 1, 1993. The retinoblastoma gene spans 180,388 nucleotides of genomic DNA (Toguchida et. al., 1993). The second parallel performance experiment involved a transcription analysis of the retinoblastoma gene using the TFD transcription factors. The retinoblastoma gene contained 20,174 transcription factor recognition sites. The parallel performance results are presented (Table 7.3.1.). The speedup curve (Figure 7.3.1.), cost curve (Figure 7.3.2.) and efficiency curve (Figure 7.3.3.) are further presented.

\begin{tabular}{|c|c|c|c|c|}
\hline processors & $\begin{array}{r}\text { time } \\
\text { (seconds) }\end{array}$ & speedup & crst & efficiency \\
\hline $\begin{array}{l}1 \\
2 \\
4 \\
8\end{array}$ & $\begin{array}{r}103.097774 \\
51.561620 \\
25.788495 \\
12.906601\end{array}$ & $\begin{array}{l}1.000000 \\
1.999506 \\
3.997821 \\
7.987988\end{array}$ & $\begin{array}{l}103.097774 \\
103.123240 \\
103.153980 \\
103.252808\end{array}$ & $\begin{array}{l}1.000000 \\
0.999753 \\
0.999455 \\
0.998499\end{array}$ \\
\hline
\end{tabular}

Table 7.3.1. Parallel performance analysis of transcription factor patterns. 


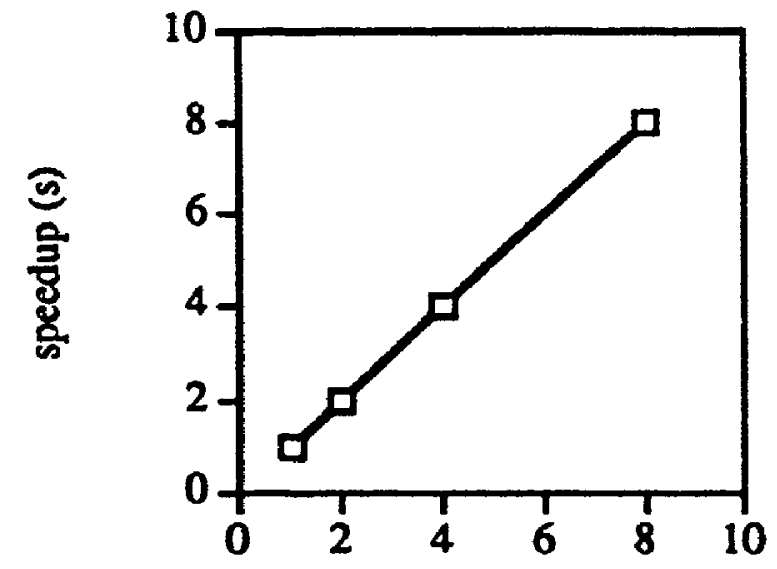

processors ( $p)$

Figure 7.3.1. Speedup curve of transcription factor patterns. The ideal speedup curve $s=p$ is plotted as a dashed line for comparison.

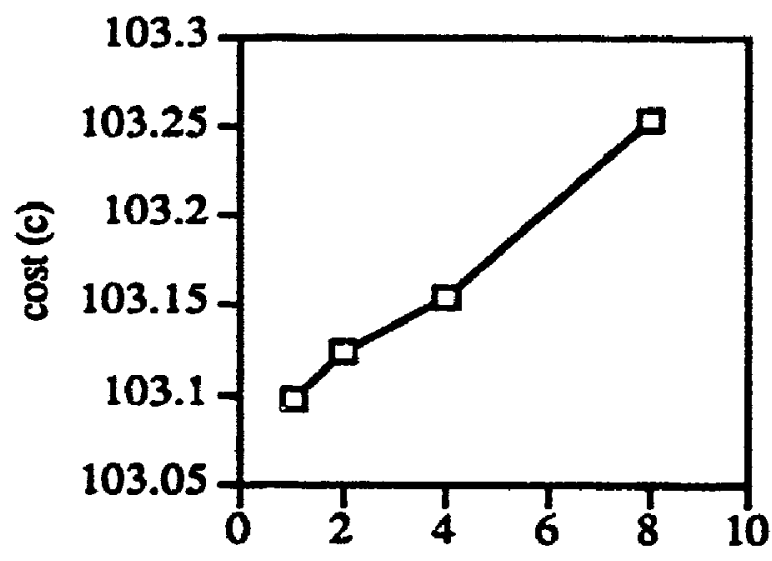

processors (p)

Figure 7.3.2. Cost curve of transcription factor patterns. 


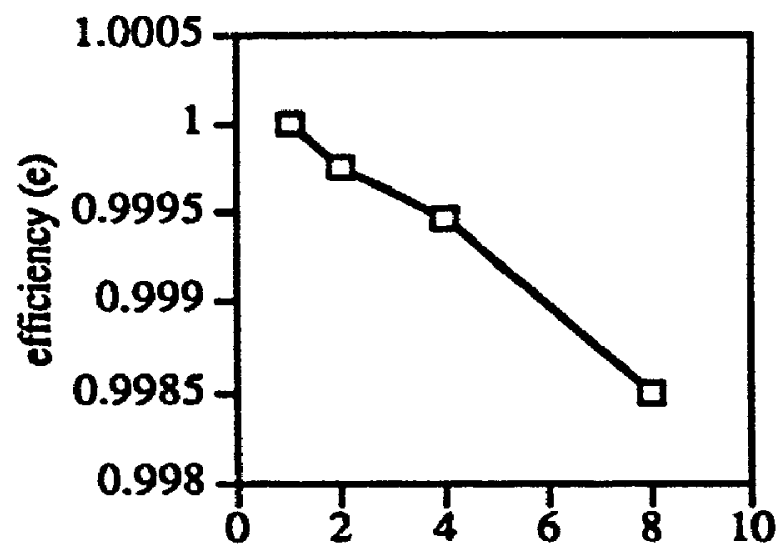

processors (p)

Figure 7.3.3. Efficiency curve of transcription factor patterns. 


\subsection{Human Interferon Transcription Factors}

The Human Interferon Transcription Factors Database (HuIFNTFD) is a comprehensive list of known human interferon transcription factor and promoter element recognition sequences. The retinoblastoma gene spans 180,388 nucleotides of genomic DNA (Toguchida et. al., 1993). The third parallel performance experiment involved a transcription analysis of the retinoblastoma gene using the HuIFNTFD transcription factors. The retinoblastoma gene contained 16,662 human interferon transcription factor recognition sites. The parallel performance results are presented (Table 7.4.1.). The speedup curve (Figure 7.4.1.), cost curve (Figure 7.4.2.) and eff. ency curve (Figure 7.4.3.) are further presented.

$\begin{array}{rrrrr}\text { processors } & \begin{array}{r}\text { time } \\ \text { (seconds) }\end{array} & \text { speedup } & \text { cost efficiency } \\ & & & & \\ 1 & 13.588732 & 1.000000 & 13.588732 & 1.000000 \\ 2 & 6.795848 & 1.999564 & 13.591696 & 0.999782 \\ 4 & 3.399615 & 3.997138 & 13.598460 & 0.999285 \\ 8 & 1.701392 & 7.986832 & 13.611136 & 0.998354\end{array}$

Table 7.4.1. Parallel performance analysis of human interferon transcription factor patterns. 


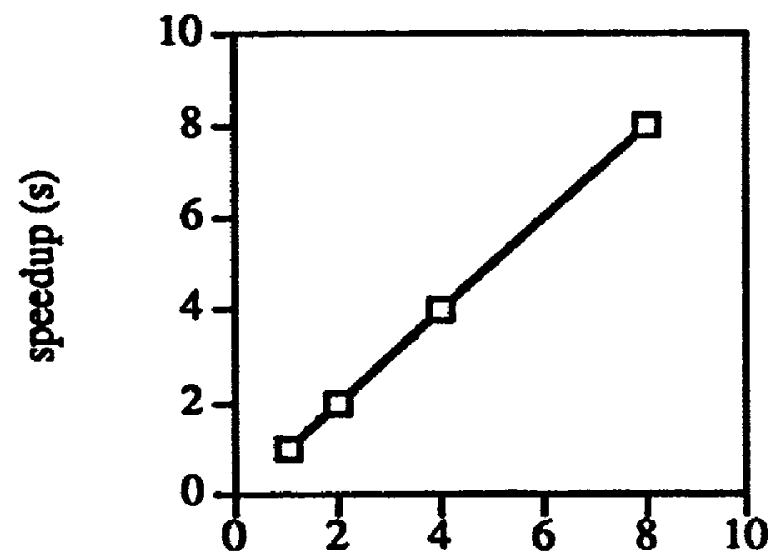

processors ( $p)$

Figure 7.4.1. Speedup curve of human interferon transcription factor patterns. The ideal speedup curve $s=p$ is plotted as a dashed line for comparison.

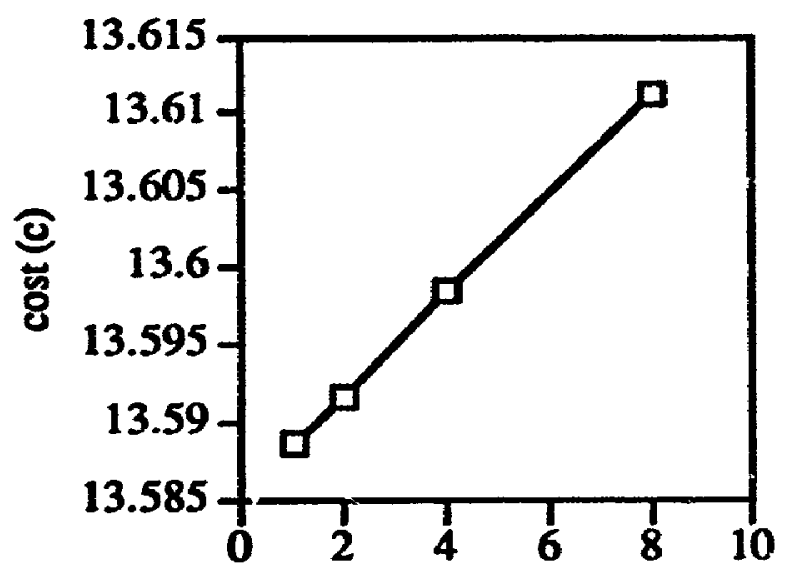

processors (p)

Figure 7.4.2. Cost curve of human interferon transcription factor patterns. 


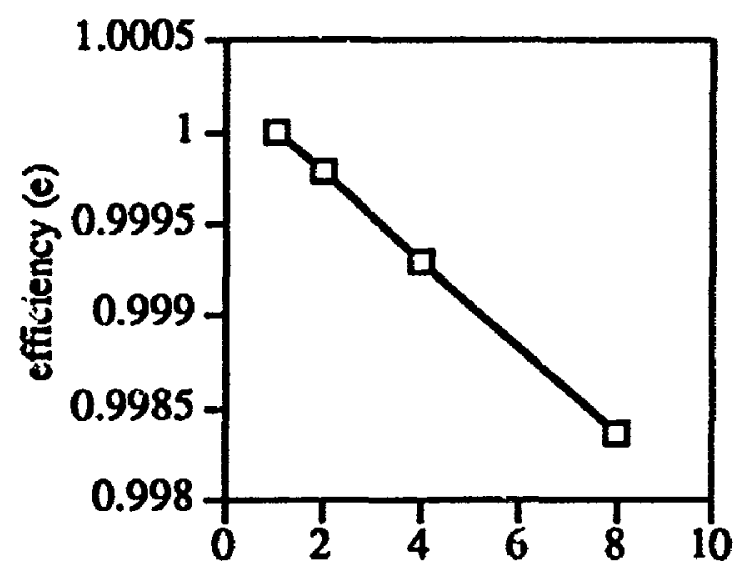

procensors (p)

Figure 7.4.3. Efficiency curve of human interferon transcription factor patterns. 


\subsection{Performance Analysis}

The parallel performance experiments did not include I/O requirements within the elapsed times. Thus, the parallel algorithm performance was evaluated on a dedicated Intel iPSC/860 Hypercube. The dedicated machine strategy avoids the reduced performance that results when a program must contend for system resources with unrelated programs. Competition between unrelated programs for limited machine resources commonly occurs in time-sharing systems. Competition for network queueing resources to perform $\mathrm{NO}$ is referred to as the $I / O$ bottleneck. Parallelism climinates the CPU bottleneck only to expose a secondary I/O bottleneck. This points out the need for high 1/O rates to match the high computation rates of parallel computers. Thus, 1/O should be performed in parallel as well.

Examination of the restriction endonuclease patterns speedup curve (Figure 7.2.1.), transcription factor patterns speedup curve (Figure 7.3.1.) and human interferon transcription factor patterns speedup curve (Figure 7.4.1.) reveals near-linear speedups in all three parallel performance experiments using a small number of processors.

Properties of the parallel performance metrics have been studied. For example, given a parallel architecture and a problem instance of fixed size, the parallel algorithm speedup does not indefinitely increase with an increasing number of processors. Speedup curves eventually exhibit saturation kinetics. For a fixed problem size, the speedup achieved by a parallel algorithm is limited by two factors: 1 . parallel algorithm overhead grows with an increasing number of processors and 2 . number of processors eventually exceeds the degree of concurrency inherent in the algorithm. 1. Parallel algorithm overhead. Certain parallel algorithm overheads are independent of the problem size, c.g. process creation. Other parallel algorithm overheads increase with the problem size but at a slower rate than the grain size, e.g. input/output and process synchronization. 2. Problem size. If there is not enough work to be done by the number of processors 
available, then the parallol algorithm demonstrates constrained speedup. This factor gives rise to a phenomenon referred to as the Amdahl effect (Goodman and Hedetniemi, 1977). The Amdahl effect explains why speedup is a nondecreasing function of input problem size.

Given a parallel algorithm and an arbitrary number of processors $\mathbf{p}$, speedup arbitrarily close to $\mathrm{p}$ can be obtained by executing the parallel algorithm on problem instances of sufficiently large size. The isoefficiency function is a scalability metric that relates problem size to the number of processors necessary for a proportional increase in speedup. In scalaile parallel algorithms, efficiency levels can be maintained at some fixed value (between 0 and 1) for an increasing number of processors by increasing the corresponding problem size.

The scalability of a parallel algorithm on a parallel architecture is a measure of its capacity to effectively utilize an increasing number of processors. Scalability analysis is useful for three purposes. 1. Selection of the best parallel algorithm and parallel architecture combination for a problem under different problem size and processor number growth constraints. 2. Performance prediction of a parallel algorithm and parallel architecture combination from known performance on fewer processors. 3. Given a fixed problem size, determination of the optimal number of processors to be used and the maximum possible speedup to be obtained. Scalability analysis of parallel algorithms has been reviewed (Kumar and Gupta, 1994). 


\section{Chapter 8}

\section{Conclusions}

The parallel nucleotide motif search algorithm and human interferon-alpha genetic analysis are summarized. Future directions for parallel nucleotide motif search algorithm research and human interferon-alpha transcriptional regulation research are proposed.

\subsection{Summary}

In summary, a multiple keyword search algorithm was developed for searching for class 1 nucleotide motifs within DNA sequences. The serial class 1 nucleotide motif search algorithm uses a deterministic finite a.utomaton and is an adaptation of the Aho and Corasick (1975) algorithm. The serial class 1 nucleotide motif search algorithm was further modified for the hypercube architecture to form the parallel class 1 nucleotide motif search algorithm. The parallel class 1 nuclentide motif search algerittm was developed under the Single Program Multiple Data programming paradigm. The data parallel algorithm operates in $O(m+(n / p))$ time and occuries $O(n+m p)$ space, where $n$ is the number of nucleotides in the DNA sequence, $m$ is the total number of nuclsotides in the finite set of class 1 nucleotide motifs and $p$ is the number of processors forming the hypercube.

Numerous sequence analysis problems in computational molecular biology can be expressed as nucleotide motif search problems, e.g. restriction analysis and transcription analysis. Restriction analysis using nucleotide motif search leads to the unambiguous identification of restriction endonuclease recognition sites. Transcription analysis using nucleotide motif search suffers from the problem of low selectivity, i.e. the inability to 
exclude false positives. Problems in the prediction of transcription factor recognition sites have been reviewed (Wingender et. al., 1990).

The parallel class 1 nucleotide motif search algorithm was implemented on the Intel iPSC/860 Hypercube. Using the Intel iPSC/860 Hypercube implementation, the human interferon-alpha multigene family was characterized by nucleotide motif search analysis. Nucleotide motif search analysis was performed using restriction endonuclease and transcription factor patterns. Nucleotide motif search analysis of the human interferonalpha genes yielded restriction endonuclease recognition sites and transcription factor binding sites.

The human interferon-alpha promoters are defined as the regions immediately upsp:eam from the transcription initiation site. The human interferon-alpha promoter 5 boundaries occir at positions -111 to -100 nucleotides 5 relative to the cap site. The human interferon-alpha promoter $3^{\prime}$ boundaries occur at position -1 nucleotide 5 relative to the cap site. Nucleotide motif search analysis localized nucleotide sequences of transcriptional significance to within the human interferon-alpha promoters. The human interferon-alpha promoters underwent comparative analysis using multiple sequence alignment. Multipie sequence alignment recognized several series of homologous motifs. The homologous motifs were conserved regions of potential significance as transcription factor recognition sequences. Within each human interferon-alpha promoter five potential transcription control elements were identified: 1. tata box (TB), 2. virus-response element (VRE), 3. distal interferon regulatory factor site (DIRF), 4. proximal interferon regulatory factor site (PIRF) and 5. TG sequence (TG).

On the basis of computational evidence, a theoretical model for the transcriptional regulation of human interferon-alpha gene expression was proposed. The theoretical model is composed of three transcriptional control elements: 1 . negativ $\epsilon_{\text {iegulatory domain } 1}$ (NRD1), 2. positive regulatory domain 1 (PRD1) and 3. positive regulatory domain 2 (PRD2). The theoretical model of human interferon-alpha transcriptional regulation 
operates as follows. Human interferon-alpha genes operate under negative regulation. Interferon regulatory factor 2 (IRF-2) normally binds to negative regulatory domain 1 . The repressor blocks transcription factor binding to positive regulatory domains 1 and 2 . Induction involves repressor inactivation. TG and interferon regulatory factor 1 (IRF-1) can then bind to positive regulatory domains 1 and 2 respectively. Transcription is then activated. The hypothesis is supported solely by computational evidence and requires experimental verification. The prototype model will require modification as additional transcription factor binding sites are discovered. 


\subsection{Future Research}

Future directions for parallel nucleotide motif search algorithm research and human interferon-alpha transcriptional regulation research are advanced: 1. improved multiple keyword search algorithms, 2. identification of additional transcription factor binding sites, 3. verification of regulatory domain function and boundaries, 4. isolation of regulatory proteins, 5. viral activation rates and 6. endogenous human interferon-alpha induction.

1. Improved multiple keyword search algorithms. The multiple keyword search problem was solved using a deterministic finite automaton (Aho and Corasick, 1975). Multiple keyword search algorithms with improved time complexities have been proposed. The Boyer and Moore (1977) technique was incorporated into the Commentz-Walter (1979) algorithm. The Horspool (1980) technique was used in the Baeza-Yates and Regnier (1990) algorithm. The n-gram technique was introduced to the Kim and ShaweTaylor (1992) algorithm. Future parallel nucleotide motif search algorithms should be based on these more efficient multiple keyword search algorithms.

2. Identification of additional transcription factor hinding sites. The human interferon-beta promoter has been well characterized. The human interferon-beta promoter regulatory region extends -200 nucleotides $5^{\prime}$ relative to the transcription initiation site. The promoter region contains six regulatory elements which are classified as either positive or negative according to their transcriptional effects. There are four positive regulatory elements involved in the transcriptional activation of the human interferon-beta gene: positive regulatory domains 1 to 4 (PRDI - PRDIV). There are two negative regulatory elements involved in the stable transcriptional repression of the human interferon-beta gene prior to induction: negative regulatory domains 1 and 2 (NRDI - NRDII). Human interferon-beta transcriptional regulation has been reviewed (Maniatis et. al., 1992).

The human type-I interferon category includes the interferon-alpha, interferon-beta and interferon-omega genes. The interferon-alpha, interferon-beta and interferon-omega 
genes are thought to have evolved from a single ancestral gene (Weissmann and Weber, 1986). Because the human interferon-beta and human interferon-alpha genes are related. the search for additional transcription factor binding sites within the human interferon-alpha genes should focus on the promoter region within -200 nucleotides 5 ' relative to the transcription initiation site.

3. Verification of regulatory domain function and boundaries. The negative regulatory domain should repress transcription when placed upstream of a heterologous promoter. All iegulatory domain boundaries should be resolved using deletion and mutation analysis.

4. Isolation of regulatory proteins. Several strategies are possible for identifying proteins that bind to the human interferon-alpha promoter including gel shift and footprinting experiments. Genes enconding for the regulatory proteins could be cloned by screening cDNA expression libraries. Screening a cDNA expression library using regulatory sequences would yield a large group of proteins that recognize the regulatory domains. Additional experiments would be necessary to resolve the proteins directly involved in the human interferon-alpha regulation. The TG factor has not yet been isolated or cloned (MacDonald et. al., 1990). An altemate strategy for the identification of human interferon-alpha regulatery proteins would be the sel=ctive inactivation of the genes that encode for the regulatory proteins (Maniatis et. al., 1992).

5. Viral activation rates. The viral activation rates of human interferon genes should be established. Correlaticns between differential activation rates and regulatory sequence nucleotide differences should be further investigated.

6. Endogenous human interferon-alpha induction. The pharmaceutical use of human interferon-alpha is limited by pharmacokinetic factors. Human interferon-alpha produced by recombinant DNA technology has a short plasma elimination half-life, ranging from 4 to 5 hours. Clearance of human interferon-alpha is mediated by renal tubule catabolism. The biological half life of human interferon-alpha extends for 2 to 3 days 
following administration. To compensate for rapid renal clearance, human interferon-alpha is administered every 1 to 2 days at elevated nonphysiological doses (Dorr, 1993). A better understanding of the human interferon-alpha transcription regulatory mechanism could yield alternate pharmacological strategies involving endogenous human interferon-alpha induction (Hayes and Zoon, 1994).

In conclusion, the design of more efficient parallel nucleotide motif search algorithms and the elucidation of the human interferon-alpha transcription regulatory mechanism remain open research problems. 


\section{Appendix A}

\section{Restriction Endonucleases}

The Restriction Enzyme Database (REBASE) is a comprehensive list of known restriction endonucleases (Roberts and Macelis, 1994). REBASE release 405 type II restriction endonuclease nucleotide recognition sequences are presented.

enzyme recognition sequence

$\begin{array}{ll}\text { AatI } & \text { gacgtc } \\ \text { AccI } & \text { gtmkac } \\ \text { Acil } & \text { ccgc } \\ \text { AclI } & \text { aacgtt } \\ \text { AcyI } & \text { grcgyc } \\ \text { AfIII } & \text { cttaag } \\ \text { AfIII } & \text { acrygt } \\ \text { AgeI } & \text { accggt } \\ \text { AhaIII } & \text { ttaaa } \\ \text { AluI } & \text { agct } \\ \text { AlwII } & \text { cagnnnctg } \\ \text { ApaI } & \text { gggccc } \\ \text { ApaBI } & \text { gcannnnntgc } \\ \text { Apall } & \text { gtgcac } \\ \text { ApoI } & \text { raatty } \\ \text { AscI } & \text { ggcgcgcc } \\ \text { AsuI } & \text { ggncc } \\ \text { AsuII } & \text { ttcgaa } \\ \text { AvaI } & \text { cycgrg } \\ \text { AvaII } & \text { ggwcc } \\ \text { AvaIII } & \text { atgcat } \\ \text { AvrII } & \text { cctagg } \\ \text { BaeI } & \text { acnnnngtayc } \\ \text { Ball } & \text { tggcca } \\ \text { BamHI } & \text { ggatcc } \\ \text { BbvI } & \text { gcagc } \\ \text { BbvII } & \text { gaagac } \\ \text { BceI } & \text { ccatc } \\ \text { Bce83I } & \text { cttgag } \\ \text { BcefI } & \text { acggc } \\ \text { BcgI } & \text { gcannnnnntcg } \\ \text { BcII } & \text { tgatca } \\ \text { BedI } & \text { wccggw } \\ \text { BgII } & \text { gccnnnnnggc } \\ & \\ & \end{array}$




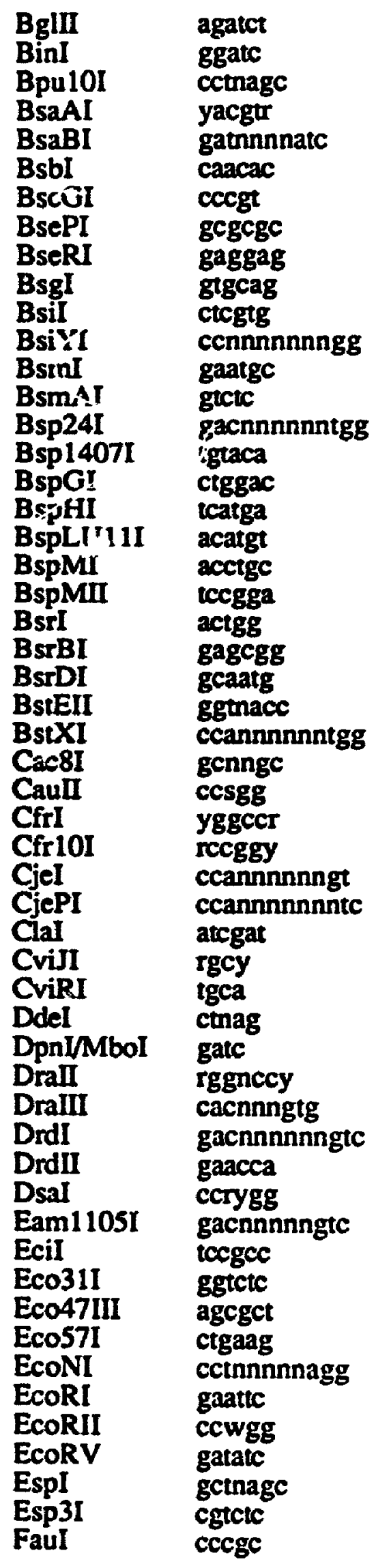




\begin{tabular}{|c|c|}
\hline $\begin{array}{l}\text { FinI } \\
\text { FnuDII } \\
\text { Fnu4HI } \\
\text { FokI } \\
\text { FseI } \\
\text { GdiII } \\
\text { GsuI } \\
\text { HaeI } \\
\text { HaeII } \\
\text { HaeIII } \\
\text { HgaI } \\
\text { HgiAI } \\
\text { HgiCI } \\
\text { HgiEII } \\
\text { HgiJII } \\
\text { HhaI } \\
\text { HindII } \\
\text { HindIII } \\
\text { HinfI } \\
\text { HpaI } \\
\text { HpaII } \\
\text { Hp!II } \\
\text { KpnI } \\
\text { Ksp632I } \\
\text { MaeI } \\
\text { MaeII } \\
\text { MaeII } \\
\text { MboII } \\
\text { McrI } \\
\text { MfeI } \\
\text { MluI } \\
\text { MmeI } \\
\text { MnII } \\
\text { MseI } \\
\text { MslI } \\
\text { MstI } \\
\text { MwoI } \\
\text { NaeI } \\
\text { NarI } \\
\text { NcoI } \\
\text { NdeI } \\
\text { NheI } \\
\text { NlaIII } \\
\text { NlaIV } \\
\text { NoII } \\
\text { NruI } \\
\text { NspI } \\
\text { NspBII } \\
\text { PacI } \\
\text { PflI108I } \\
\text { PflMI } \\
\text { PleI } \\
\text { PmaCI } \\
\text { PmeI }\end{array}$ & 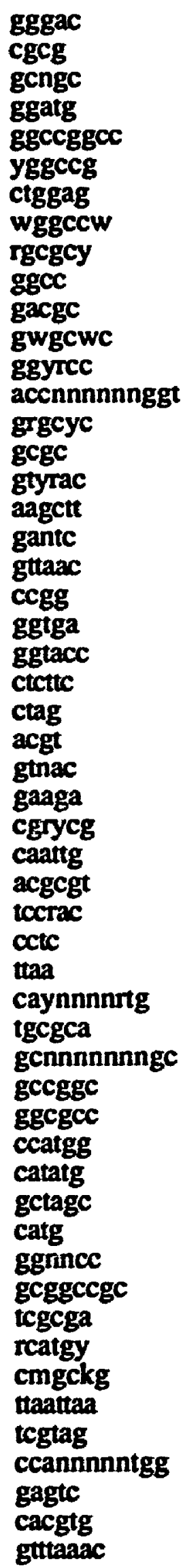 \\
\hline
\end{tabular}




\begin{tabular}{|c|c|}
\hline $\begin{array}{l}\text { PpuMI } \\
\text { PshAI } \\
\text { PstI } \\
\text { PvuI } \\
\text { PvulI } \\
\text { RleAI } \\
\text { Rsa } \\
\text { RsrII } \\
\text { SacI } \\
\text { SacII } \\
\text { Sall } \\
\text { SapI } \\
\text { SauI } \\
\text { Scal } \\
\text { ScrFI } \\
\text { SduI } \\
\text { SecI } \\
\text { SexAI } \\
\text { SfaNI } \\
\text { SfeI } \\
\text { SfiI } \\
\text { SgfI } \\
\text { SgrAI } \\
\text { Smal } \\
\text { SnaI } \\
\text { SnaBI } \\
\text { SpeI } \\
\text { SphI } \\
\text { SplI } \\
\text { SrfI } \\
\text { Sse8387I } \\
\text { SspI } \\
\text { StuI } \\
\text { StyI } \\
\text { SwaI } \\
\text { TaqI } \\
\text { TaqIIa } \\
\text { TaqIIb } \\
\text { TfiI } \\
\text { TseI } \\
\text { Tsp45I } \\
\text { TspEI } \\
\text { Tih1III } \\
\text { Tth1IIII } \\
\text { VspI } \\
\text { XbaI } \\
\text { XcmI } \\
\text { XhoI } \\
\text { XhoII } \\
\text { XmaIII } \\
\text { XmnI } \\
\end{array}$ & 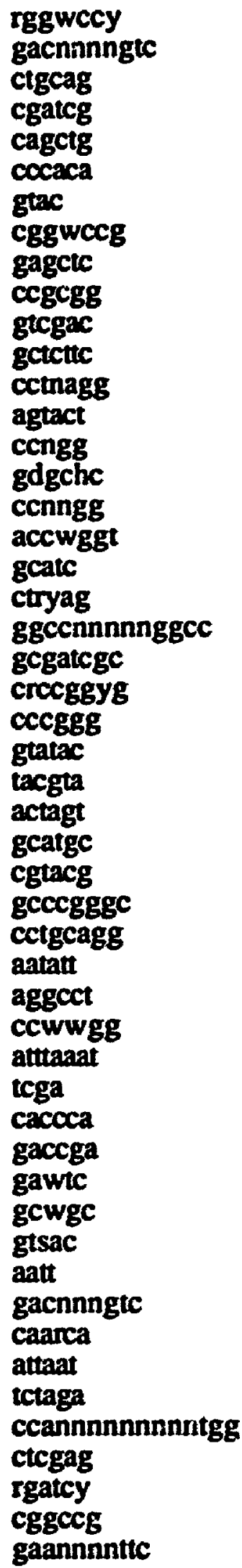 \\
\hline
\end{tabular}




\section{Appendix B}

\section{Transcription Factors}

The Transcription Factors Database (TFD) is the largest compiled list of known transcription factors (Ghosh, 1993). TFD transcription factor nucleotide recognition sequences are presented.

Enzyme Recognition Sequence

\begin{tabular}{|c|c|}
\hline 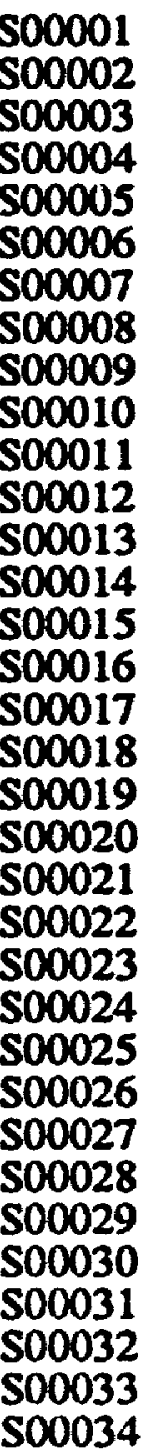 & 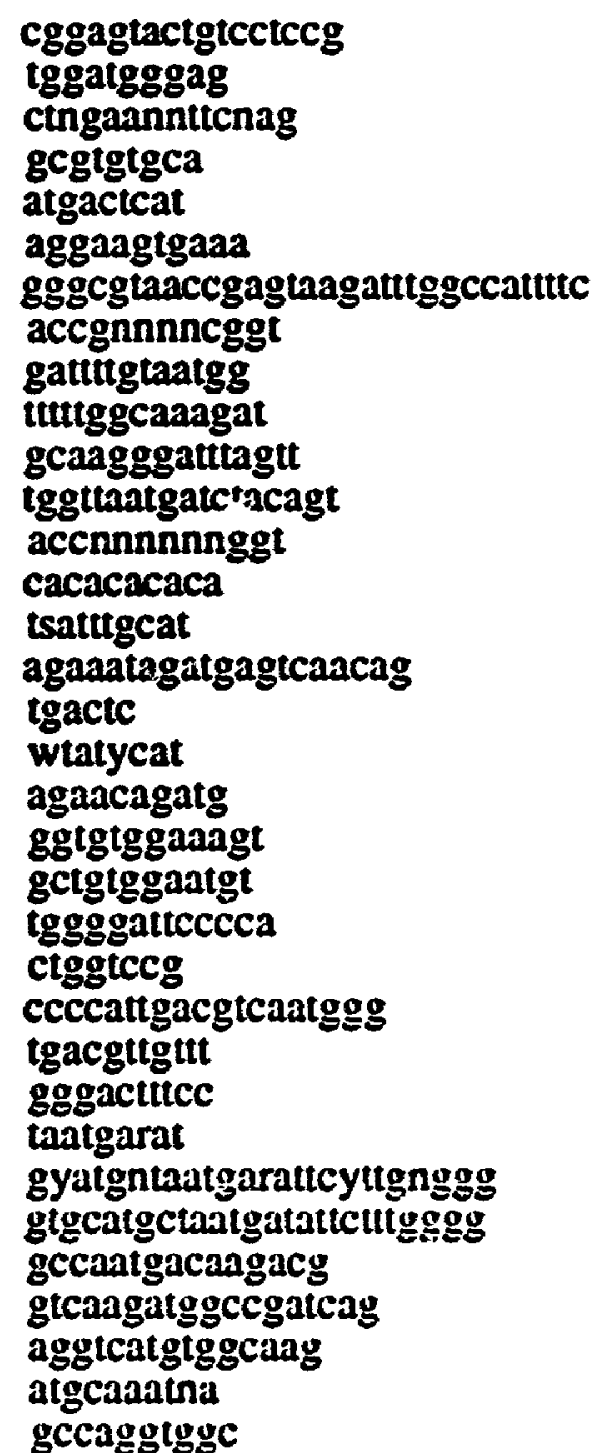 \\
\hline
\end{tabular}




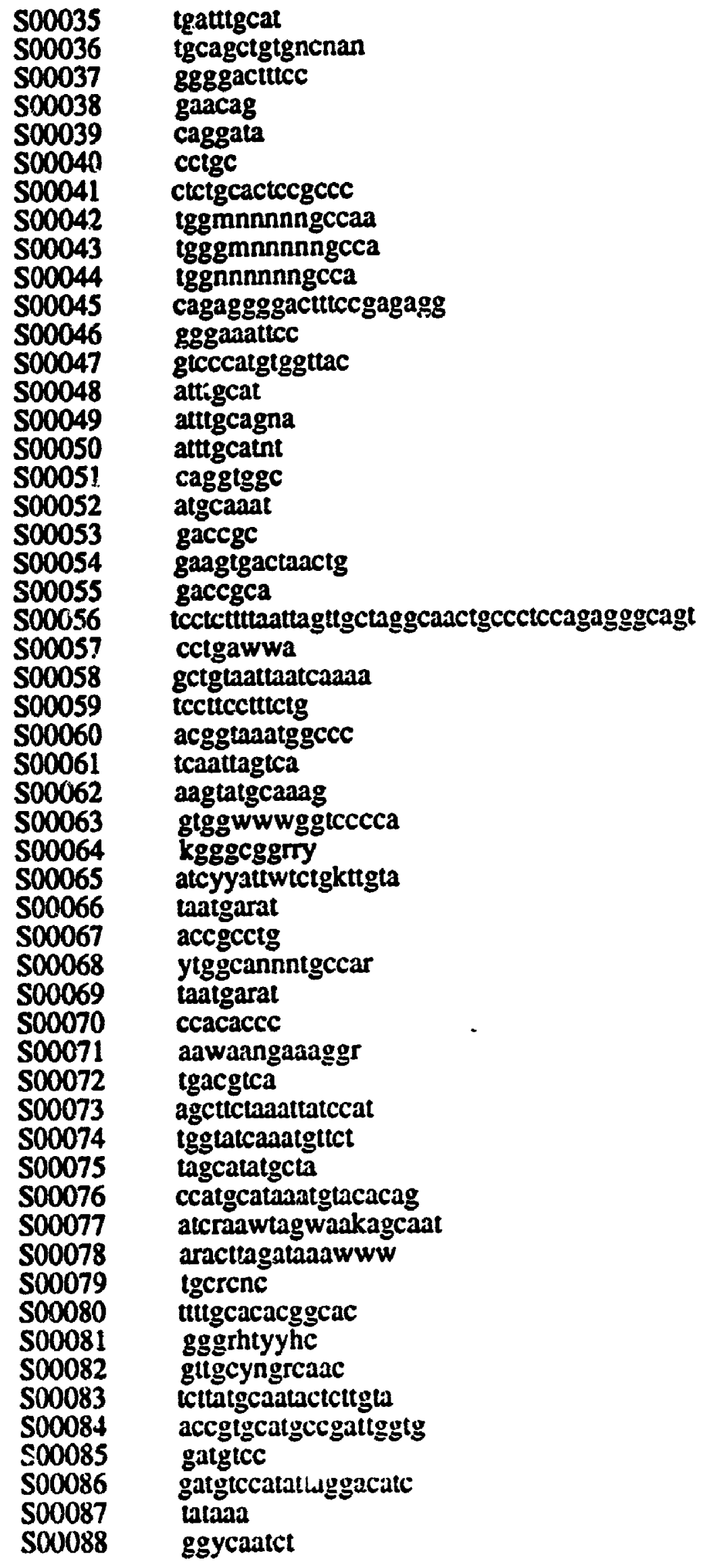




$\begin{array}{ll}\text { S00089 } & \text { canyyy } \\ \text { S00090 } & \text { gagagga } \\ \text { S00091 } & \text { actcagaggaaaa } \\ \text { S00092 } & \text { ggccacgtgacc } \\ \text { S00093 } & \text { gggwctggtcakg } \\ \text { S00094 } & \text { tccccangcg } \\ \text { S00095 } & \text { ccccaggc } \\ \text { S00096 } & \text { gggtgtggaaagtc } \\ \text { S00097 } & \text { ycagctgygg } \\ \text { S00098 } & \text { aaccaat } \\ \text { S00099 } & \text { agccact } \\ \text { S00100 } & \text { agccaat } \\ \text { S00101 } & \text { gttaatnattaac } \\ \text { S00102 } & \text { yggtwcamwtgtyct } \\ \text { S00103 } & \text { cacgaaaa } \\ \text { S00104 } & \text { gtkacgw } \\ \text { S00105 } & \text { tctccaacttataagttggaga } \\ \text { S00106 } & \text { caattaaa } \\ \text { S00107 } & \text { ttttgctggaacggttcag } \\ \text { S00108 } & \text { caactgataaggat } \\ \text { S00109 } & \text { atgcaaag } \\ \text { S00110 } & \text { atgcatct } \\ \text { S00111 } & \text { gggcgg } \\ \text { S00112 } & \text { rgttamtnntynncm } \\ \text { S00113 } & \text { tcaattaaatga } \\ \text { S00114 } & \text { aaartggaaryg } \\ \text { S00115 } & \text { tcctatca } \\ \text { S00116 } & \text { gtcatcatagacg } \\ \text { S00117 } & \text { sggtwcaanntgtyct } \\ \text { S00118 } & \text { yrgttcryttyyn } \\ \text { S00119 } & \text { atgctctaataaattt } \\ \text { S00120 } & \text { ttcctaattaggaa } \\ \text { S00121 } & \text { tknngyaak } \\ \text { S00122 } & \text { tgttcccattatgttct } \\ \text { S00123 } & \text { gagattccac } \\ \text { S00124 } & \text { tcaggta } \\ \text { S00125 } & \text { ggtcannntgacc } \\ \text { S00126 } & \text { ccaggggtcagggggggggtgctt } \\ \text { S00127 } & \text { gtgtcagtca } \\ \text { S00128 } & \text { aanccaaa } \\ \text { S00129 } & \text { tataat } \\ \text { S00130 } & \text { atccaacaatcratttaccaatc } \\ \text { S00131 } & \text { s }: \text { gtgkgtt } \\ \text { S00132 } & \text { accgaccg } \\ \text { S00133 } & \text { tctgtctg } \\ \text { S00134 } & \text { accgactg } \\ \text { S00135 } & \text { ctcgagtacgaaccgaccgtcggtagg } \\ \text { S00136 } & \text { ttatccaaaacctcggttacagg,aac } \\ \text { S00137 } & \text { aaaactacgtcatctcca } \\ \text { S00138 } & \text { tcgcgcccttct } \\ \text { S00139 } & \text { gtnwayattnatnng } \\ \text { S00140 } & \text { tataaa } \\ \text { S00141 } & \text { gagtgatgactcacgtcaag } \\ \text { S00142 } & \text { agaaccaatgggcac } \\ & \end{array}$




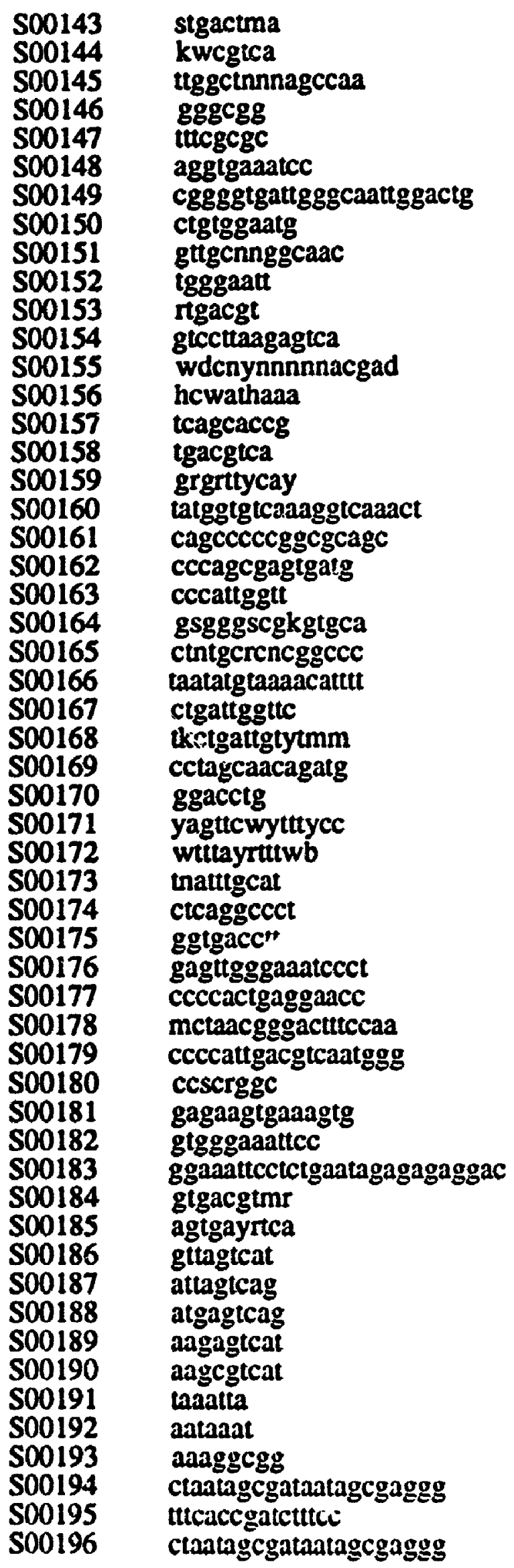




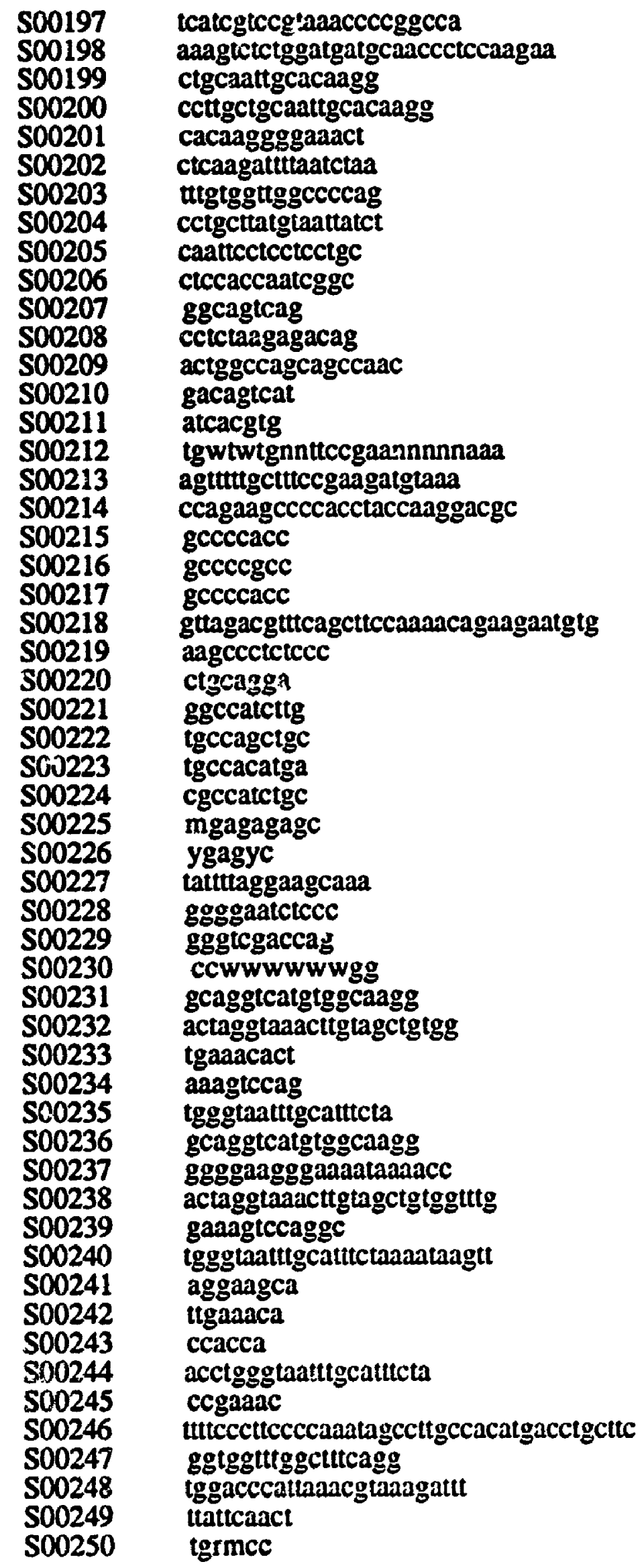




$\begin{array}{ll}\text { S00251 } & \text { tggttaat } \\ \text { S00252 } & \text { ctgatta } \\ \text { S00253 } & \text { tgaggtgtcgaaggt } \\ \text { S00254 } & \text { aacctccgttatctccat } \\ \text { S00255 } & \text { acattctcctgccaag } \\ \text { S00256 } & \text { ccckcccwcct } \\ \text { S00257 } & \text { cctctccagattacattcatc } \\ \text { S00258 } & \text { scccacctc } \\ \text { S00259 } & \text { ssccccagnntc } \\ \text { S00260 } & \text { ctccyctgycc } \\ \text { S00261 } & \text { ctaaatac } \\ \text { S00262 } & \text { tcntactc } \\ \text { S00263 } & \text { taatactc } \\ \text { S00264 } & \text { tctactc } \\ \text { S00265 } & \text { acctactc } \\ \text { S06266 } & \text { taagactc } \\ \text { S00267 } & \text { tcttaagc } \\ \text { S00268 } & \text { ttccacac } \\ \text { S00269 } & \text { tcctaccc } \\ \text { S00270 } & \text { tcatacca } \\ \text { S00271 } & \text { gttagggtgtggaaagtccc } \\ \text { S00272 } & \text { aggatatctgtggtaagca } \\ \text { S00273 } & \text { agtgtggtttgcaagaggaa } \\ \text { S00274 } & \text { gccattg } \\ \text { S00275 } & \text { rgggrmtyycc } \\ \text { S00276 } & \text { ggggacttcc } \\ \text { S00277 } & \text { agggacttcc } \\ \text { S00278 } & \text { gggggattcc } \\ \text { S00279 } & \text { ggggaatccc } \\ \text { S00280 } & \text { ggggaatctcc } \\ \text { S00281 } & \text { gccaatc } \\ \text { S00282 } & \text { tggattgaagccaa } \\ \text { S00283 } & \text { tgggcacagtgccaa } \\ \text { S00284 } & \text { tggcttgggecaa } \\ \text { S00285 } & \text { tggatttgtgccaa } \\ \text { S00286 } & \text { tggaaggcagccaa } \\ \text { S00287 } & \text { tggaaggtatccaa } \\ \text { S00288 } & \text { tggcactatgccac } \\ \text { S00289 } & \text { tgggcagattgccaa } \\ \text { S00290 } & \text { tggcaagctgtcaa } \\ \text { S00291 } & \text { tggattgaggccaa } \\ \text { S00292 } & \text { tggcactgggccaa } \\ \text { S00293 } & \text { tggcacatggccaa } \\ \text { S00294 } & \text { tggctaatggccaa } \\ \text { S00295 } & \text { tggccaatagccaa } \\ \text { S00296 } & \text { tggacatgagccaa } \\ \text { S00297 } & \text { tggctatatgccaa } \\ \text { S00298 } & \text { tggattgaagccaat } \\ \text { S00299 } & \text { catccgtggctggagccaaaagtg } \\ \text { S00300 } & \text { atttggattgaagccaatatga } \\ \text { S00301 } & \text { gccaatga } \\ \text { S00303 } & \text { accccgcca } \\ \text { S00304 } & \text { tggattgaagccaata } \\ \text { tgacgtca } \\ & \end{array}$




\begin{tabular}{|c|c|}
\hline 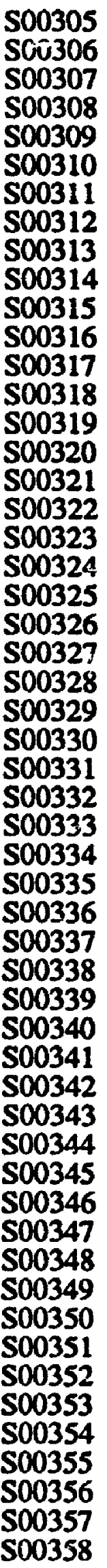 & 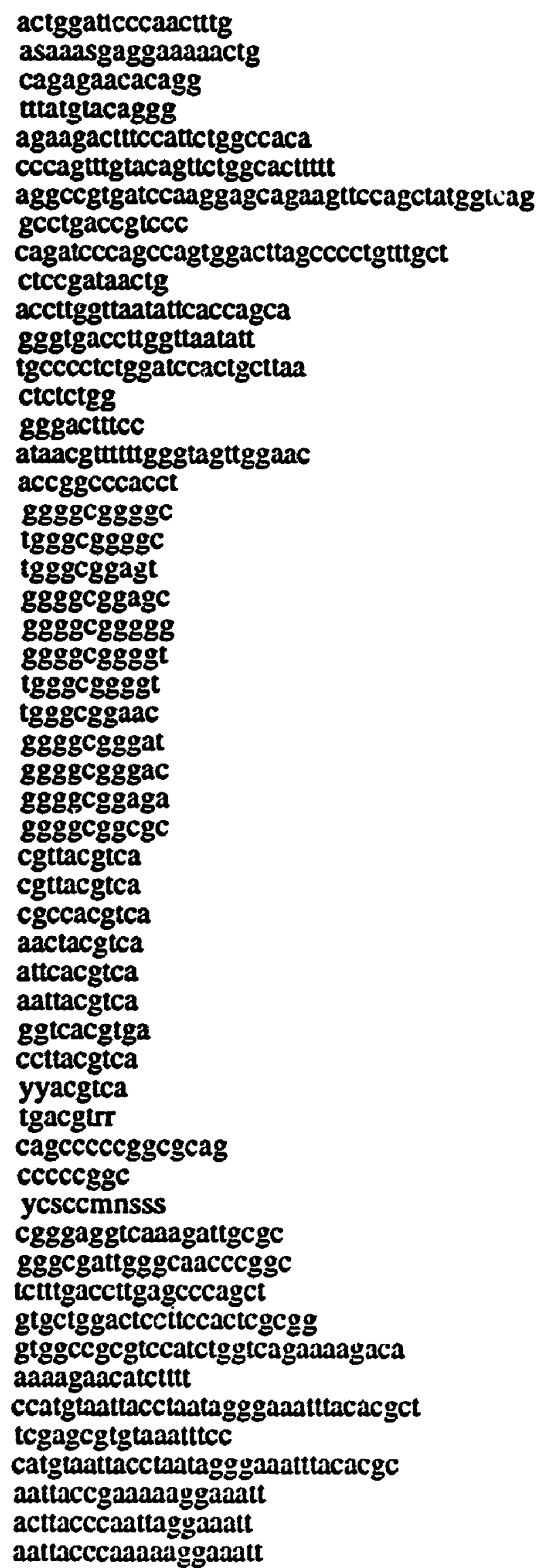 \\
\hline
\end{tabular}




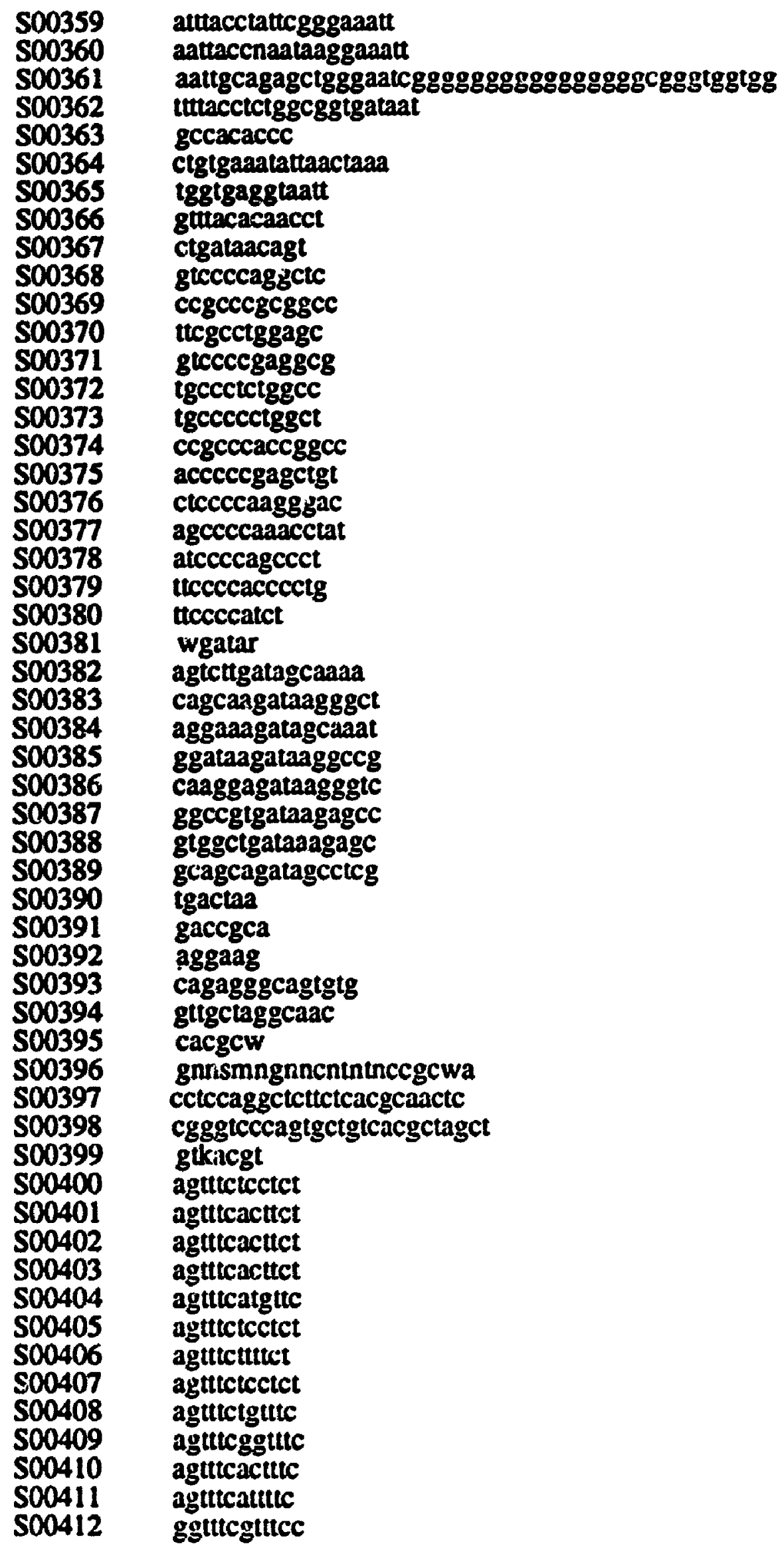




\begin{tabular}{|c|c|}
\hline $\begin{array}{l}\text { S00413 } \\
\text { S00414 } \\
\mathbf{S} 00415 \\
\mathbf{S} 00416 \\
\mathbf{S} 00417 \\
\mathbf{S} 00418 \\
\mathbf{S} 00419 \\
\mathbf{S} 00420 \\
\mathbf{S} 00421 \\
\mathbf{S} 00422 \\
\mathbf{S} 00423 \\
\mathbf{S} 00424 \\
\mathbf{S} 00425 \\
\mathbf{S} 00426 \\
\mathbf{S} 00427 \\
\mathbf{S} 00428 \\
\mathbf{S} 00429 \\
\mathbf{S} 00430 \\
\mathbf{S} 00431 \\
\mathbf{S} 00432 \\
\mathbf{S} 00433 \\
\mathbf{S} 00434 \\
\mathbf{S} 00435 \\
\mathbf{S} 00436 \\
\mathbf{S} 00437 \\
\mathbf{S} 00438 \\
\mathbf{S} 00439 \\
\mathbf{S} 00440 \\
\mathbf{S} 00441 \\
\mathbf{S} 00442 \\
\mathbf{S} 00443 \\
\mathbf{S} 00444 \\
\mathbf{S} 00445 \\
\mathbf{S} 00446 \\
\mathbf{S} 00447 \\
\mathbf{S} 00448 \\
\mathbf{S} 00449 \\
\mathbf{S} 00450 \\
\mathbf{S} 00451 \\
\mathbf{S} 00452 \\
\mathbf{S} 00453 \\
\mathbf{S} 00454 \\
\mathbf{S} 00455 \\
\mathbf{S} 00456 \\
\mathbf{S} 00457 \\
\mathbf{S} 00458 \\
\mathbf{S} 00459 \\
\mathbf{S} 00460 \\
\mathbf{S} 00461 \\
\mathbf{S} 00462 \\
\mathbf{S} 00463 \\
\mathbf{S} 00464 \\
\mathbf{S} 00465 \\
\mathbf{S} 00466 \\
\end{array}$ & 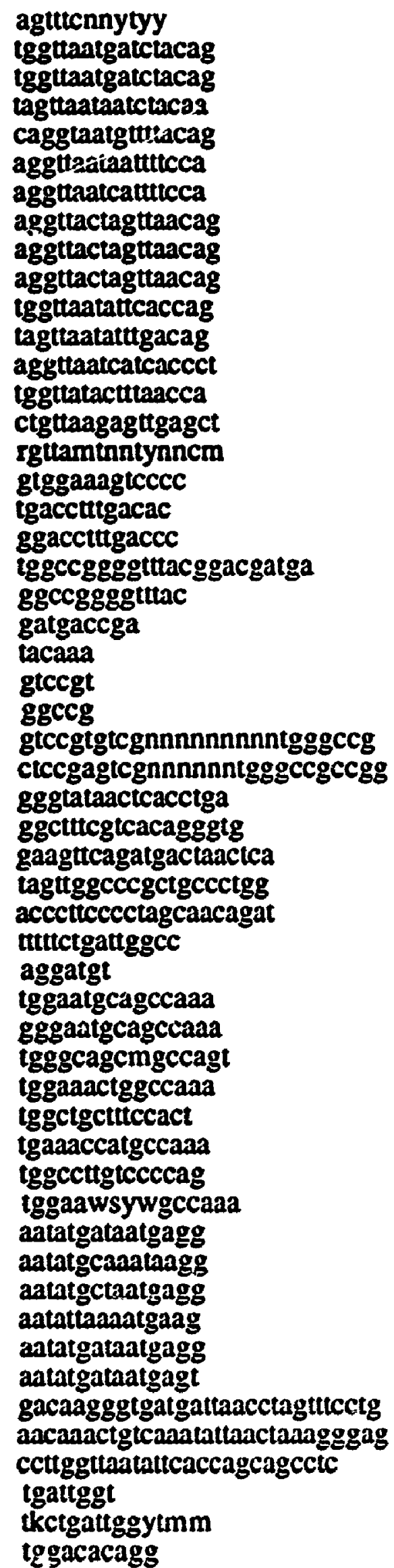 \\
\hline
\end{tabular}




\begin{tabular}{|c|c|}
\hline 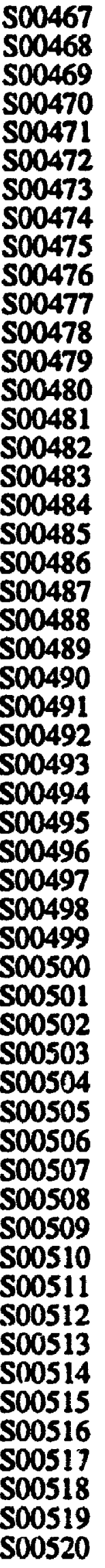 & 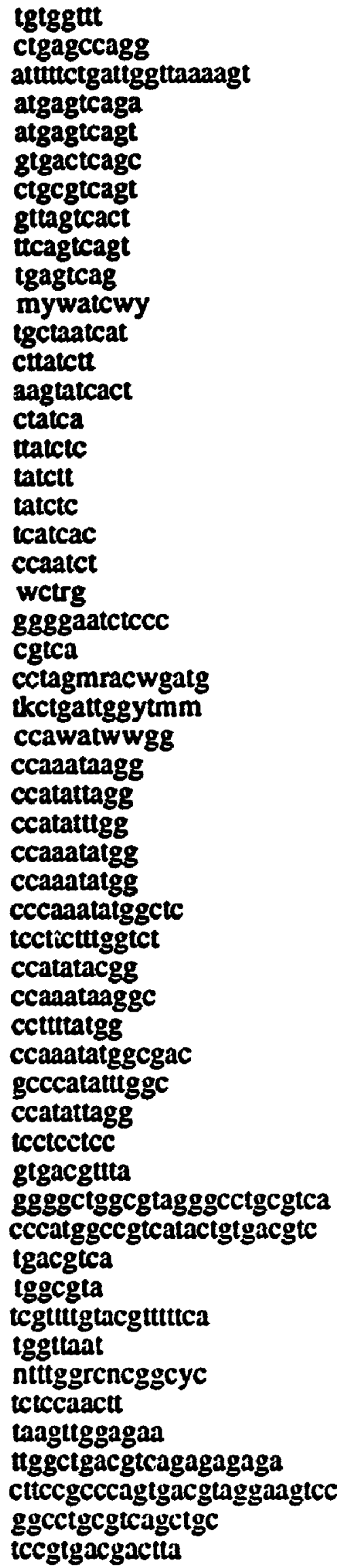 \\
\hline
\end{tabular}




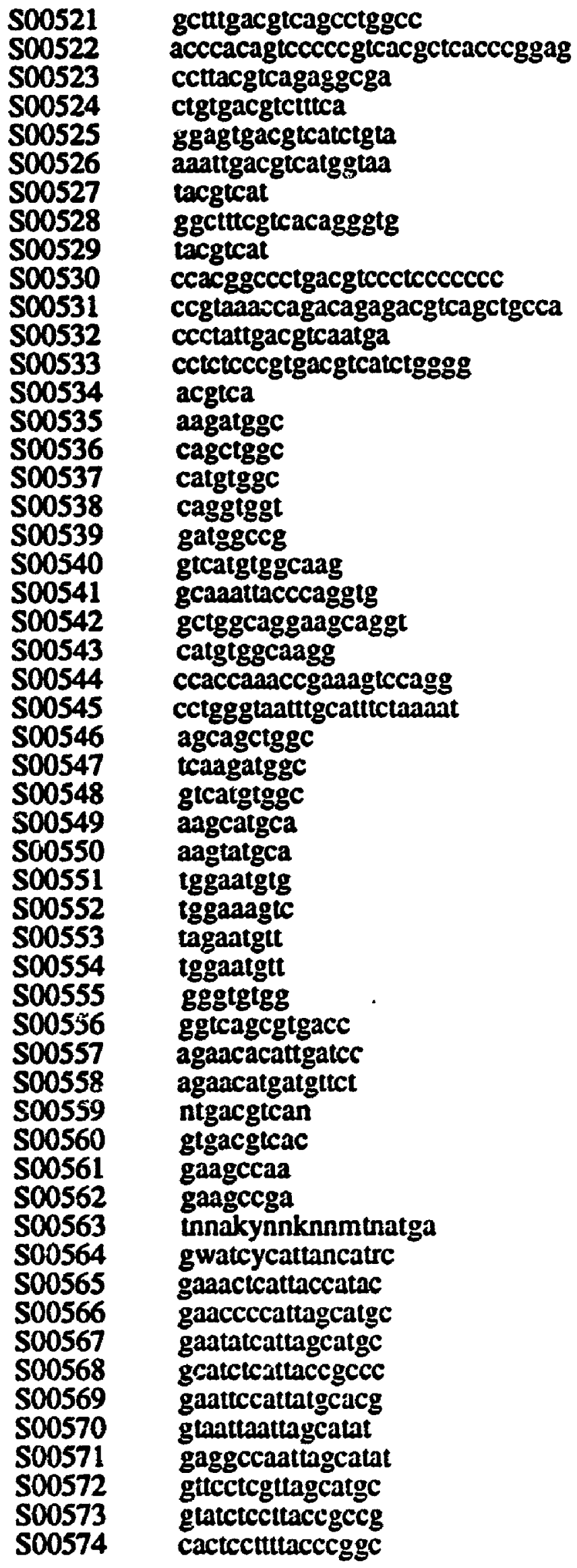




\begin{tabular}{|c|c|}
\hline 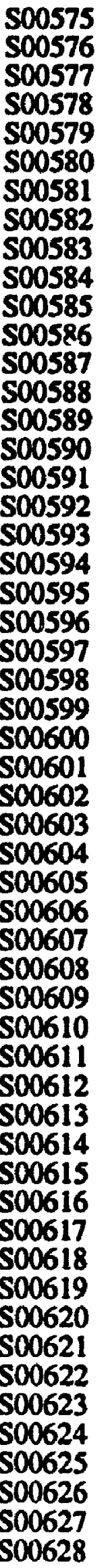 & 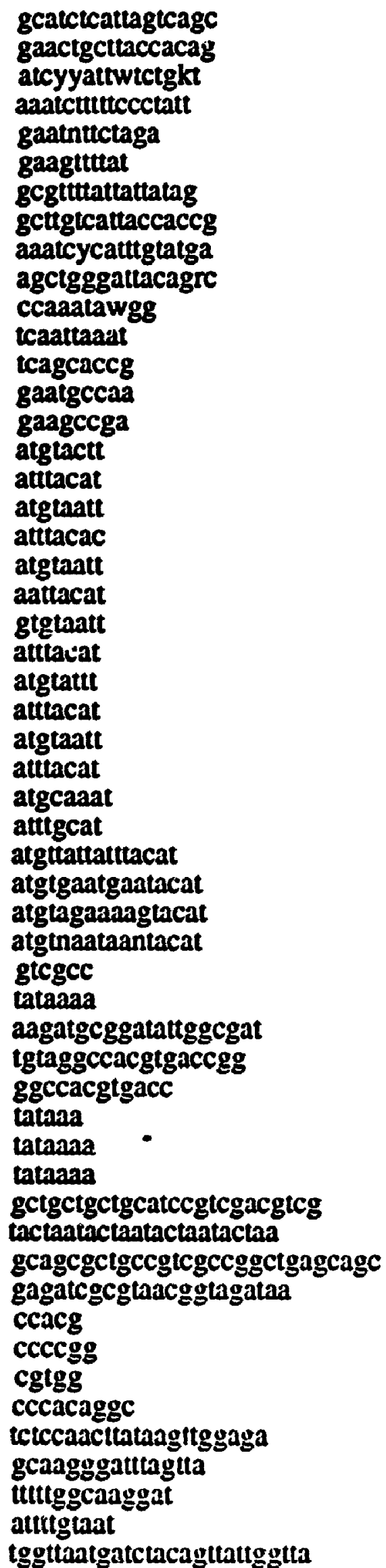 \\
\hline
\end{tabular}




\begin{tabular}{|c|c|}
\hline 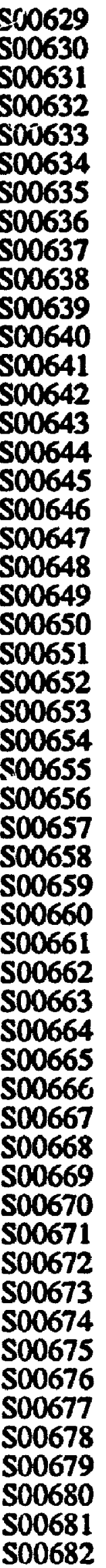 & 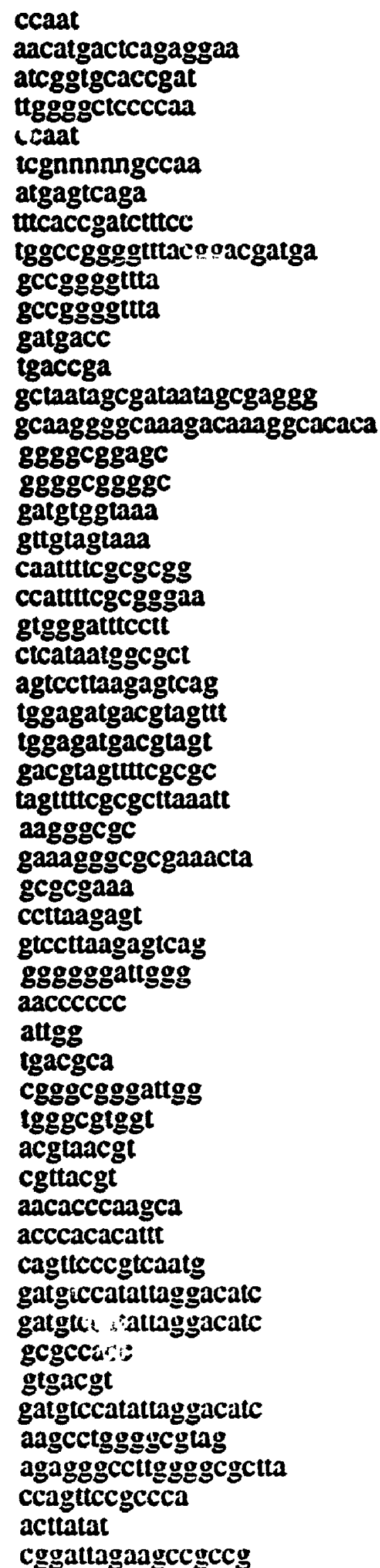 \\
\hline
\end{tabular}




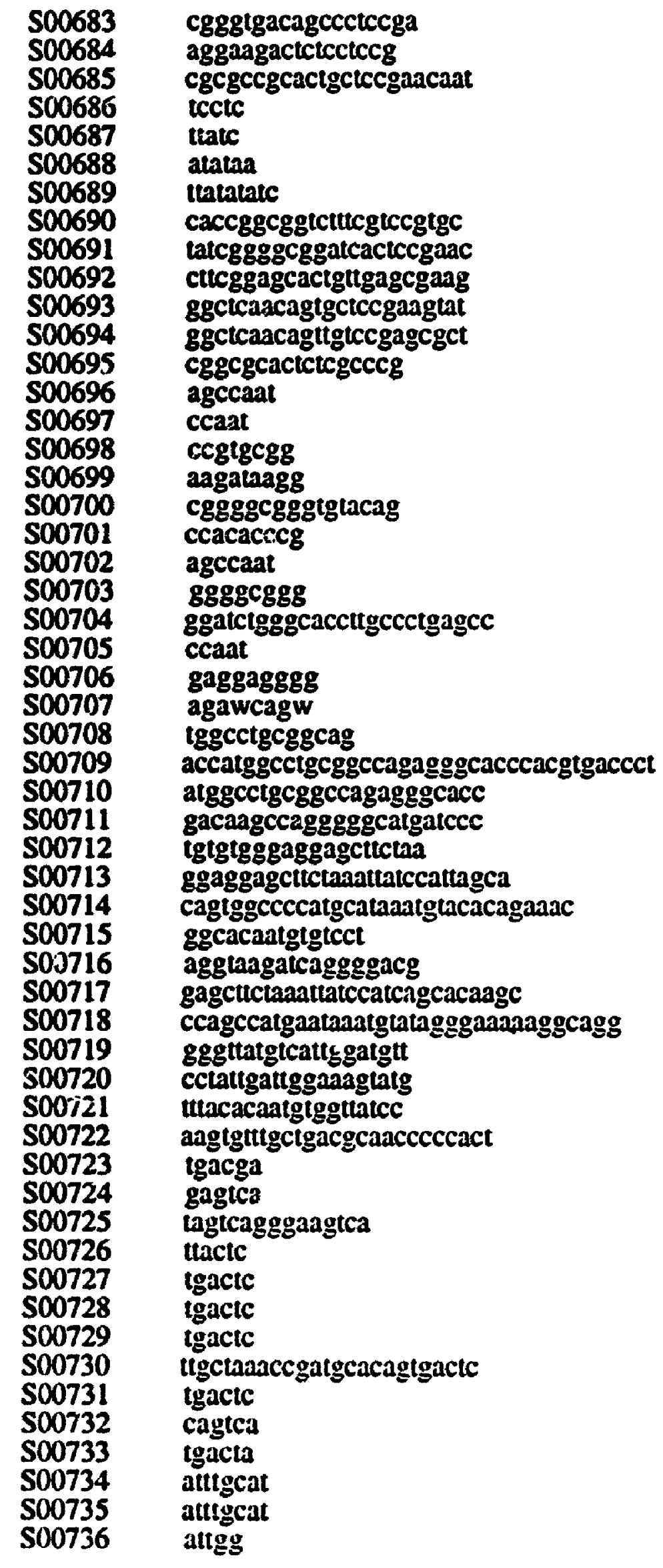




\begin{tabular}{|c|c|}
\hline 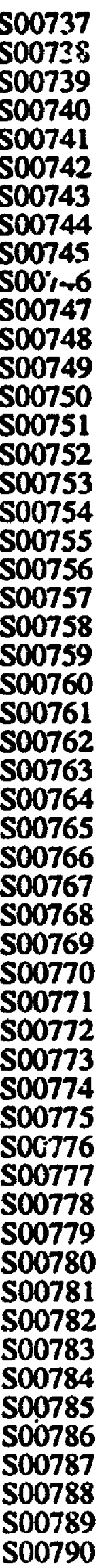 & 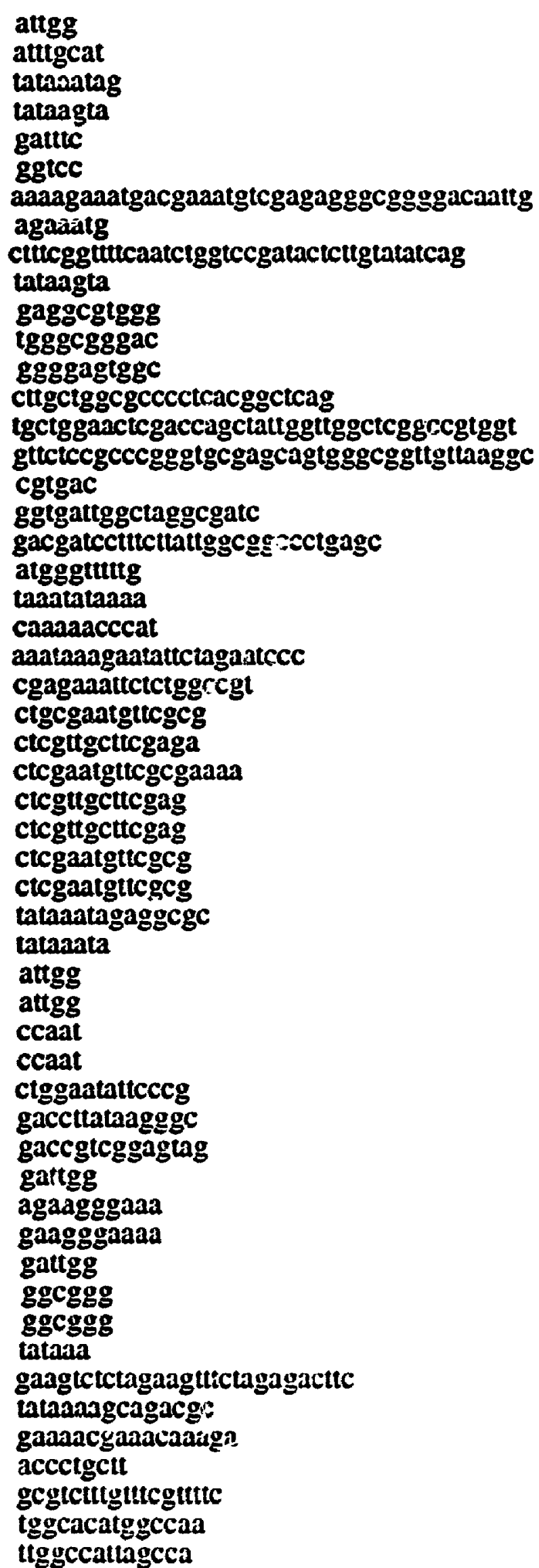 \\
\hline
\end{tabular}




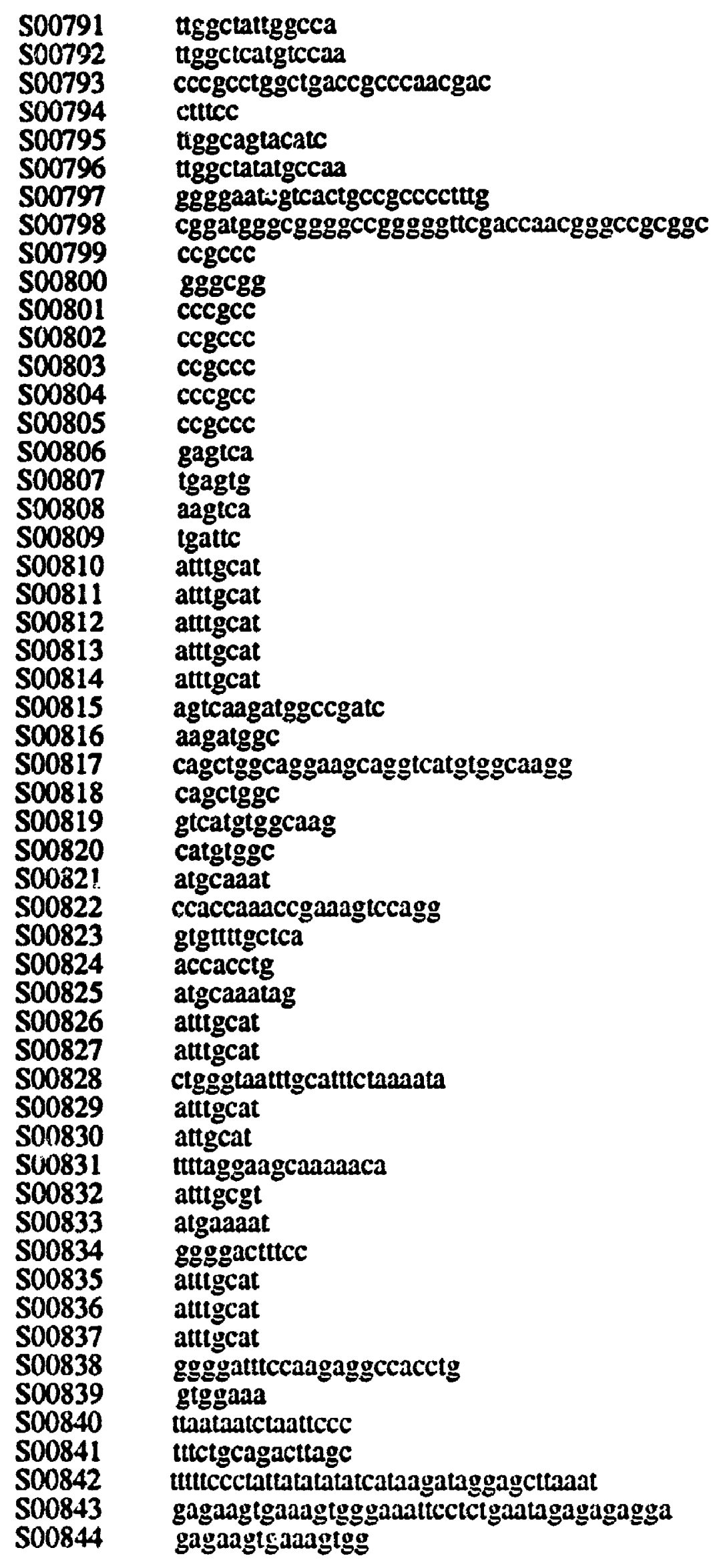




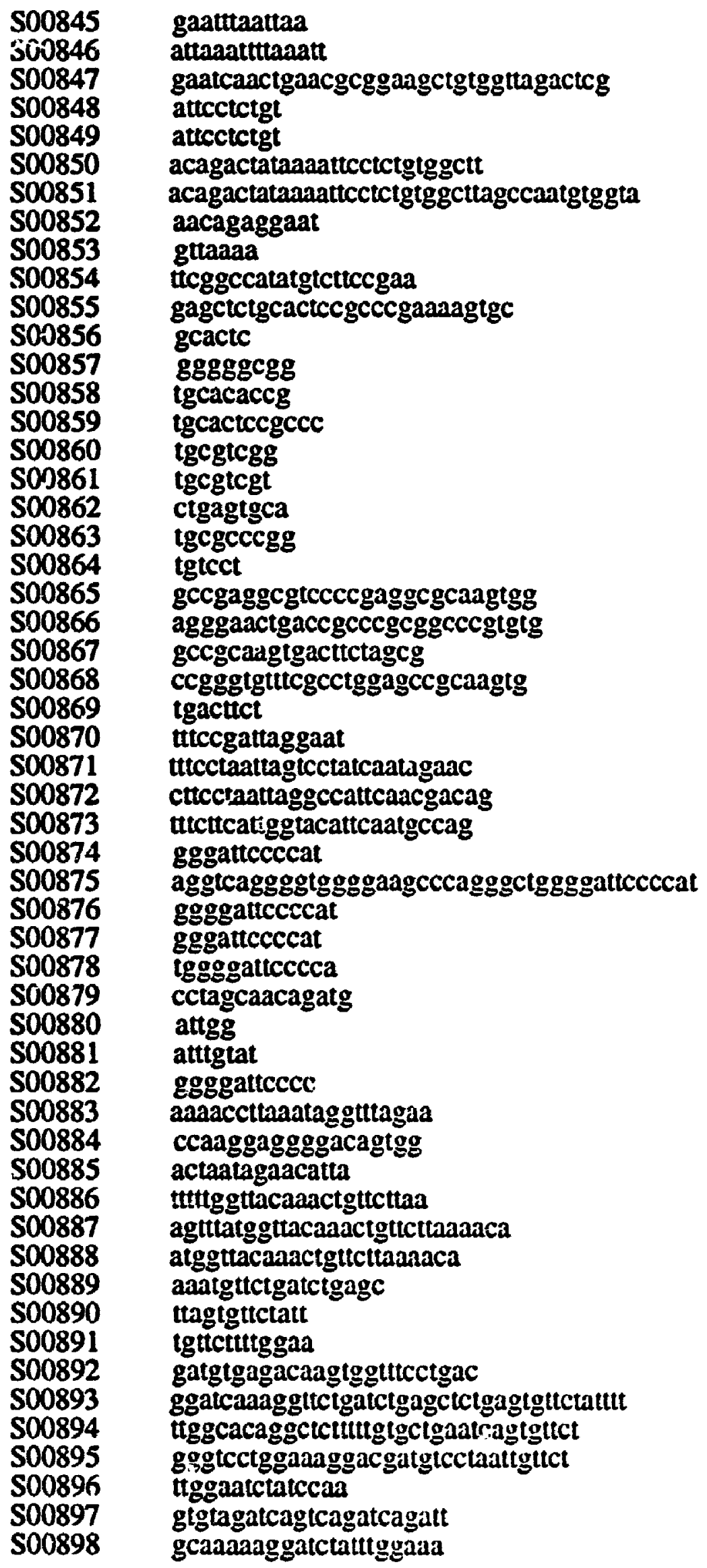




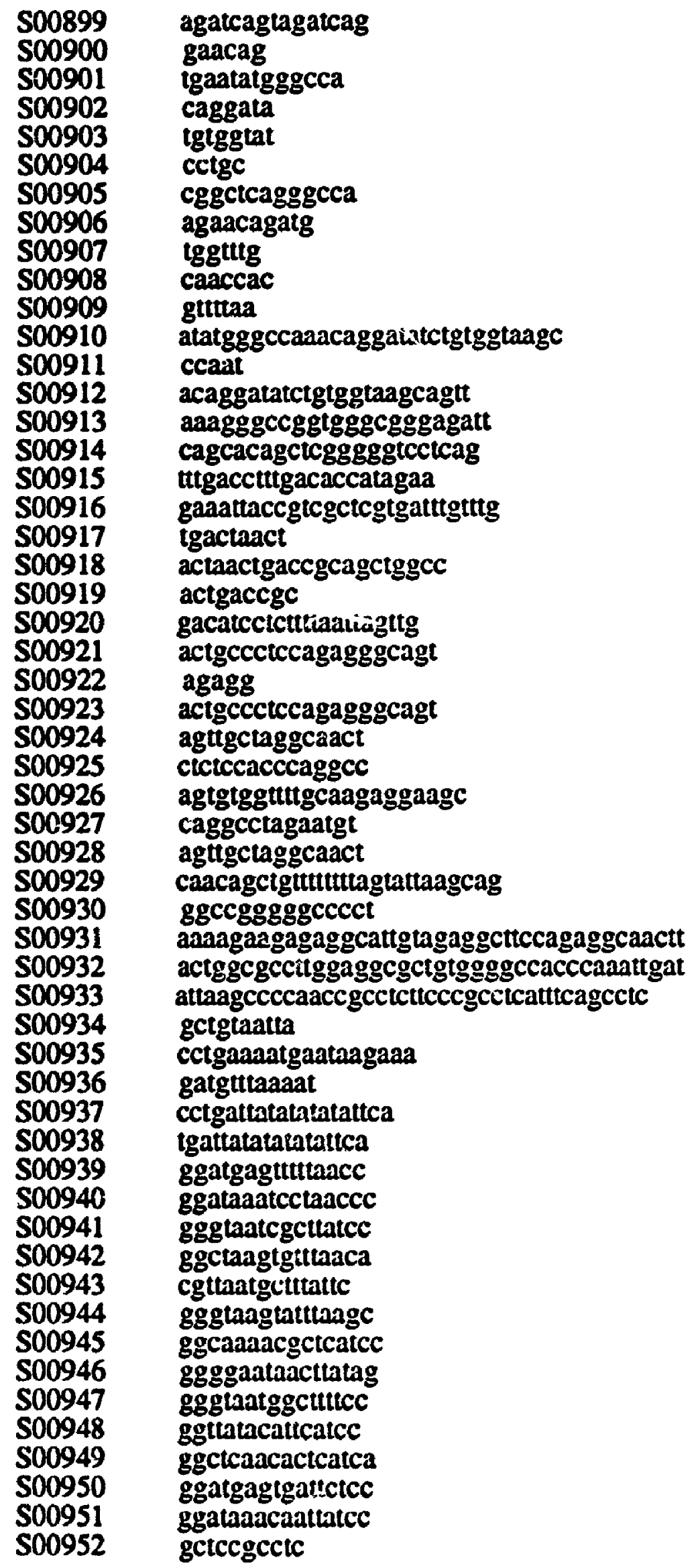




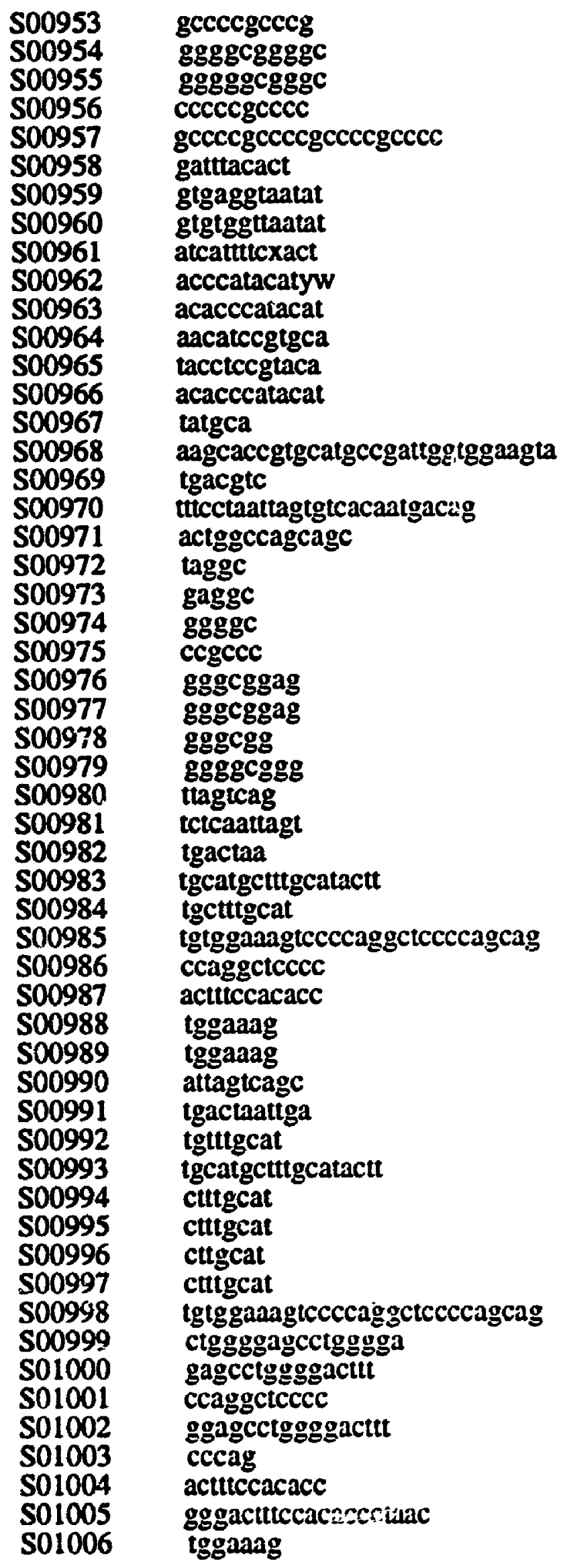




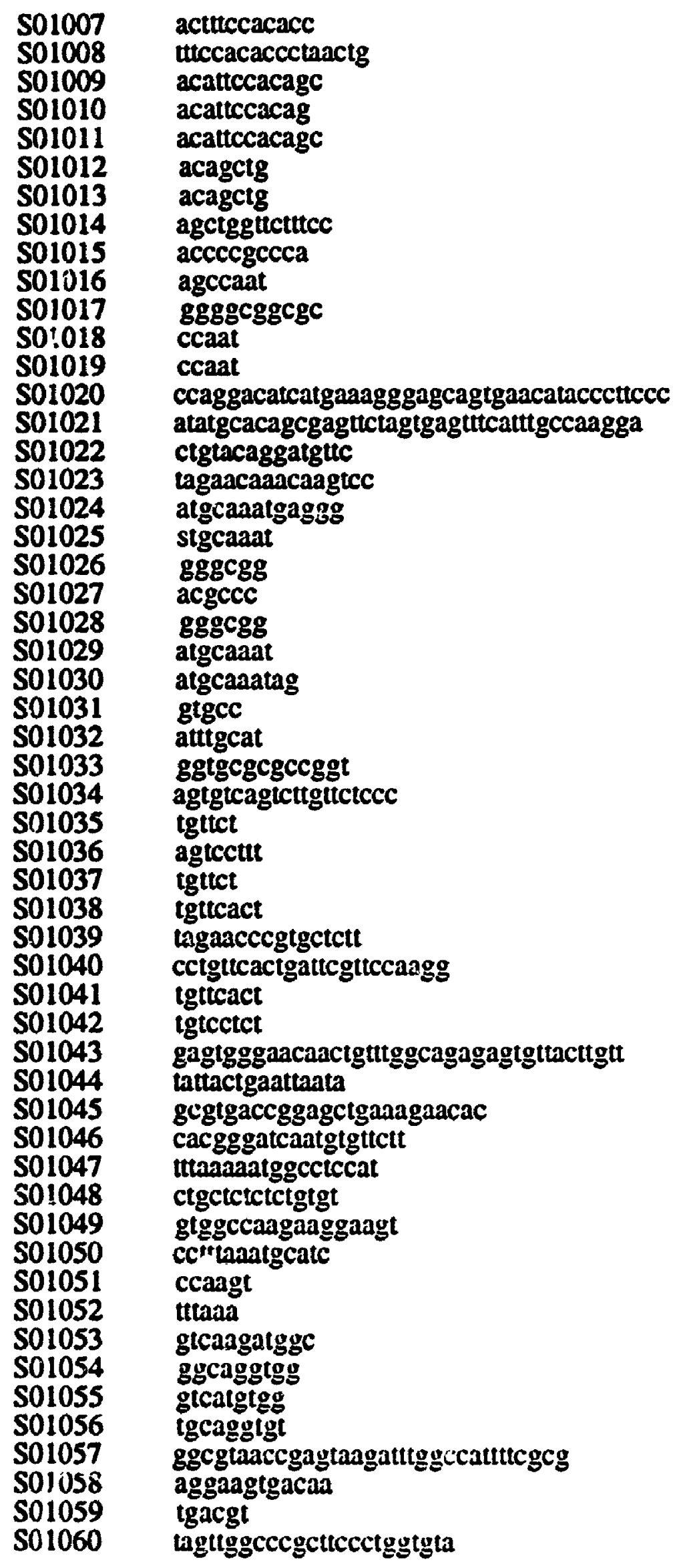




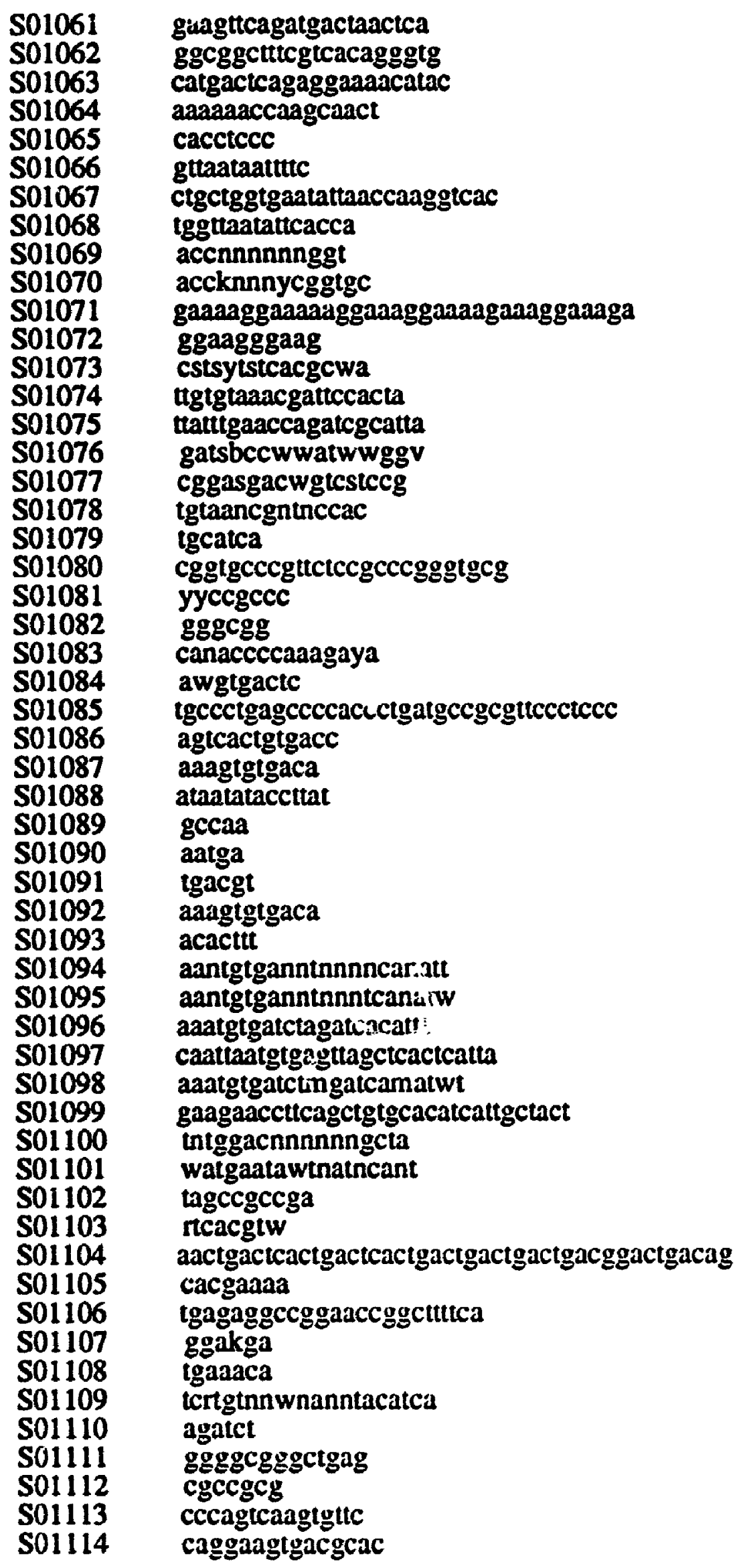




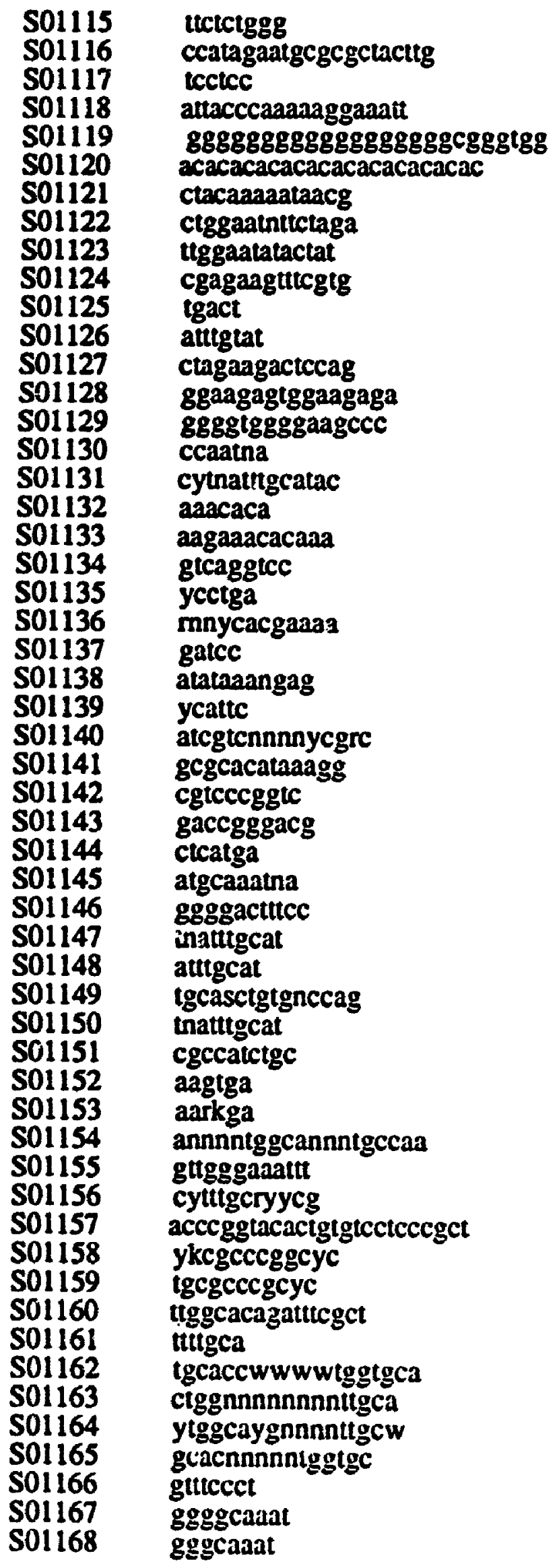




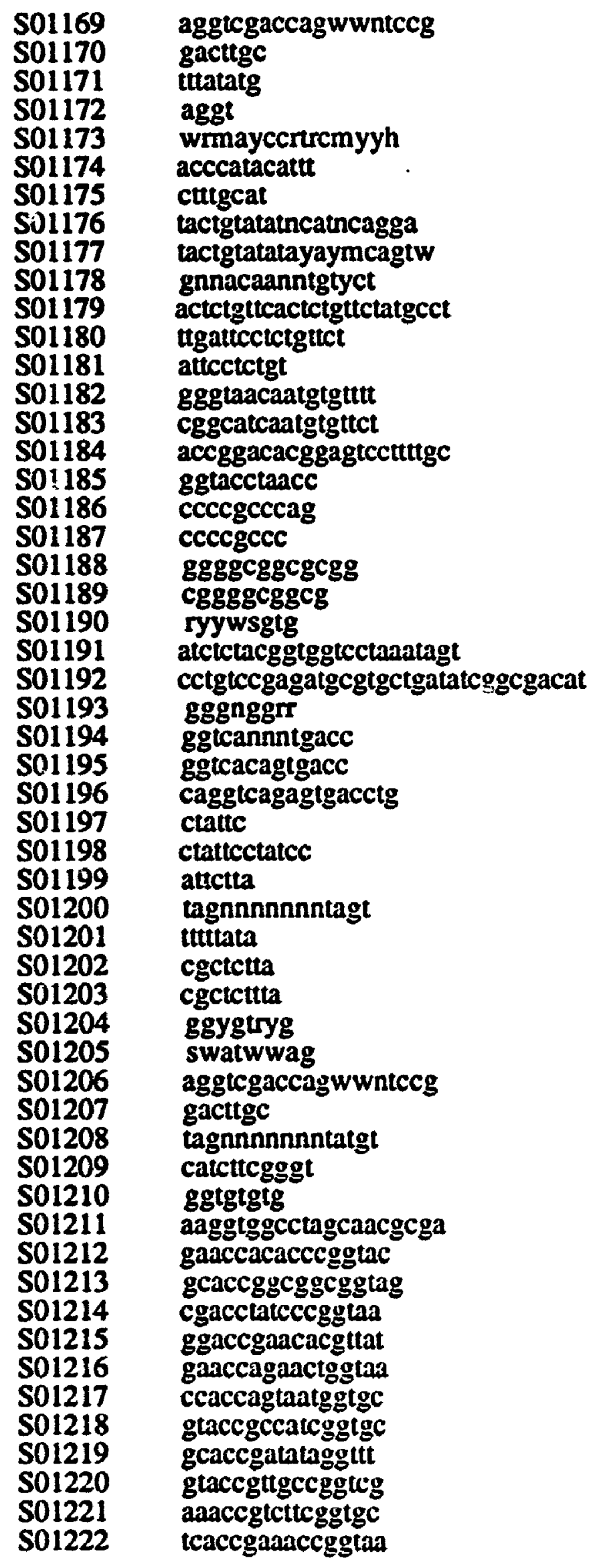




\begin{tabular}{|c|c|}
\hline 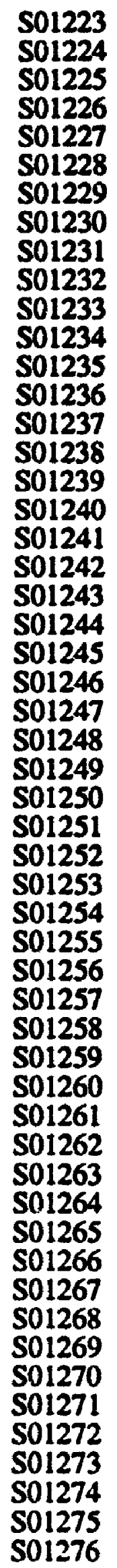 & 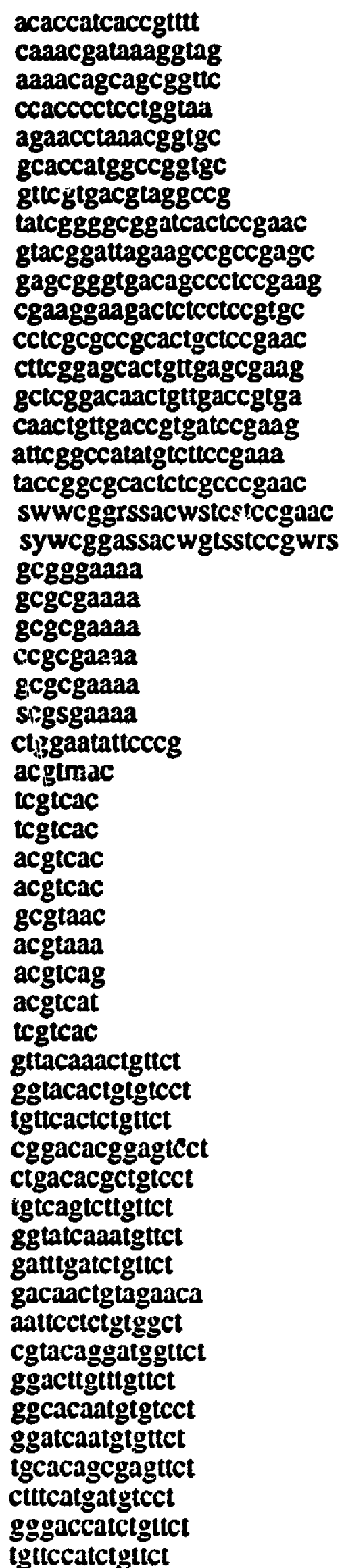 \\
\hline
\end{tabular}




\begin{tabular}{|c|c|}
\hline 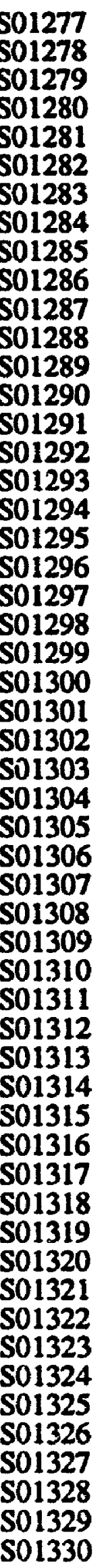 & 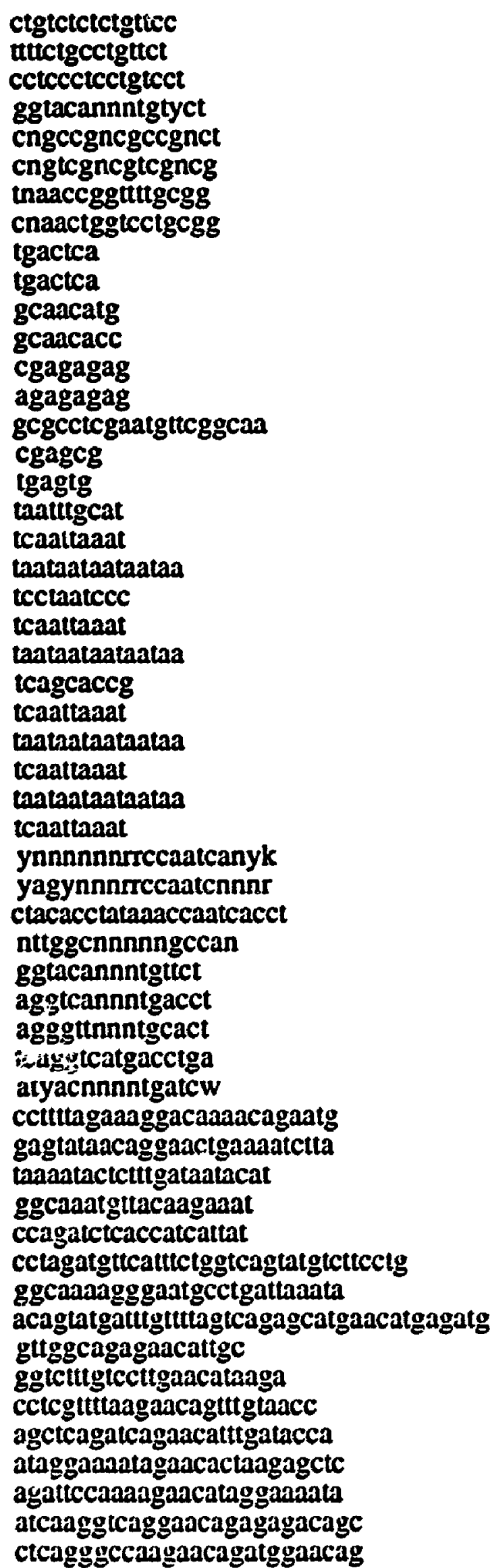 \\
\hline
\end{tabular}




\begin{tabular}{|c|c|}
\hline 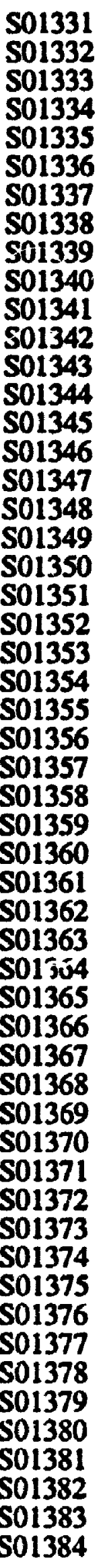 & 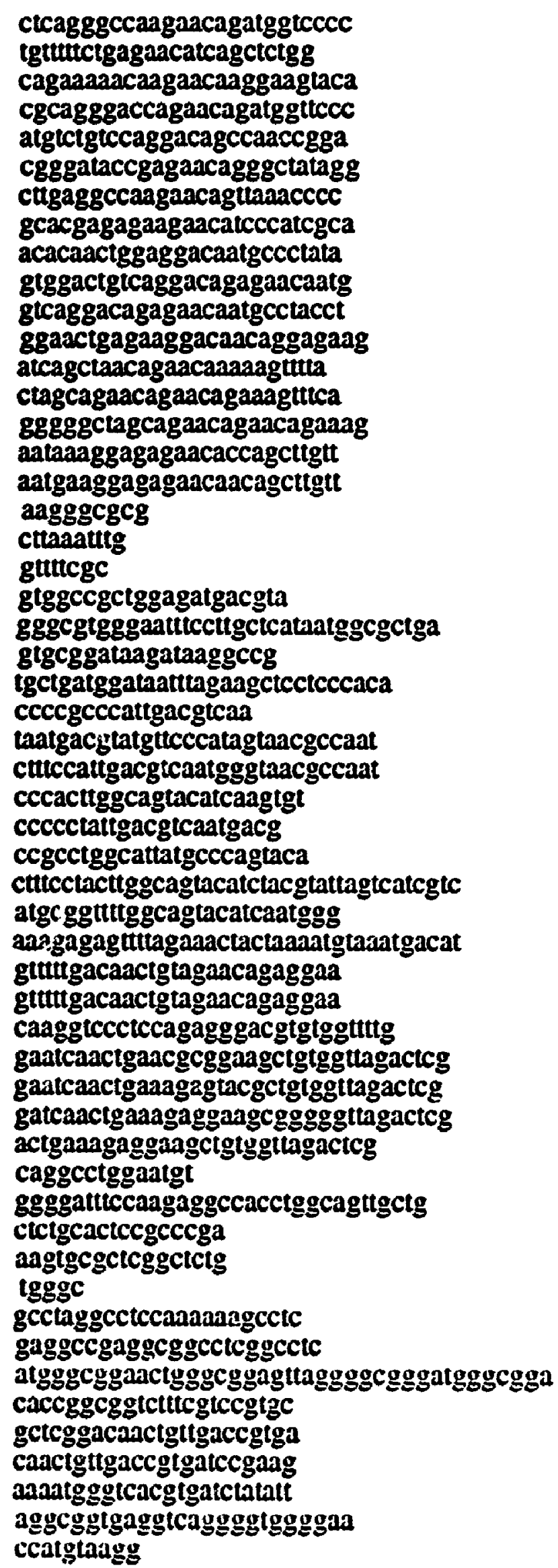 \\
\hline
\end{tabular}




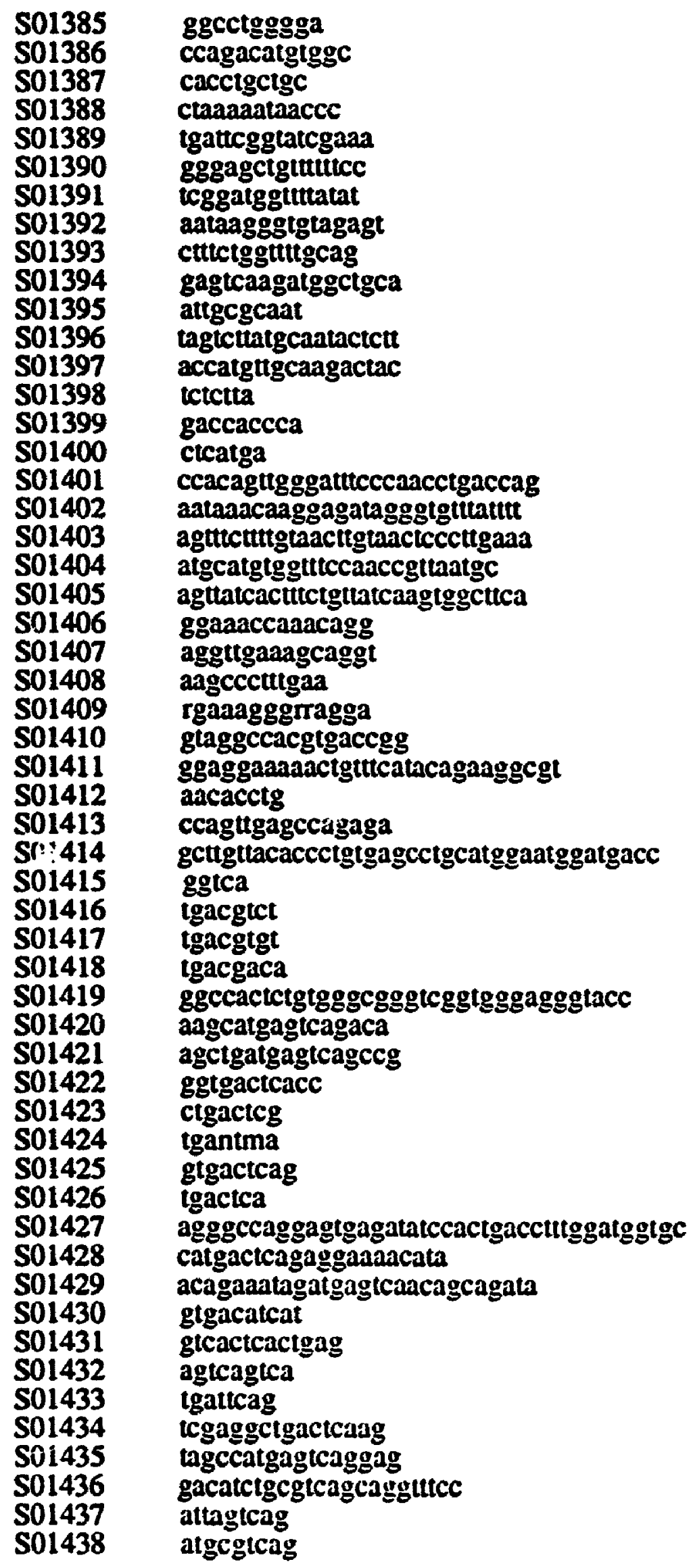




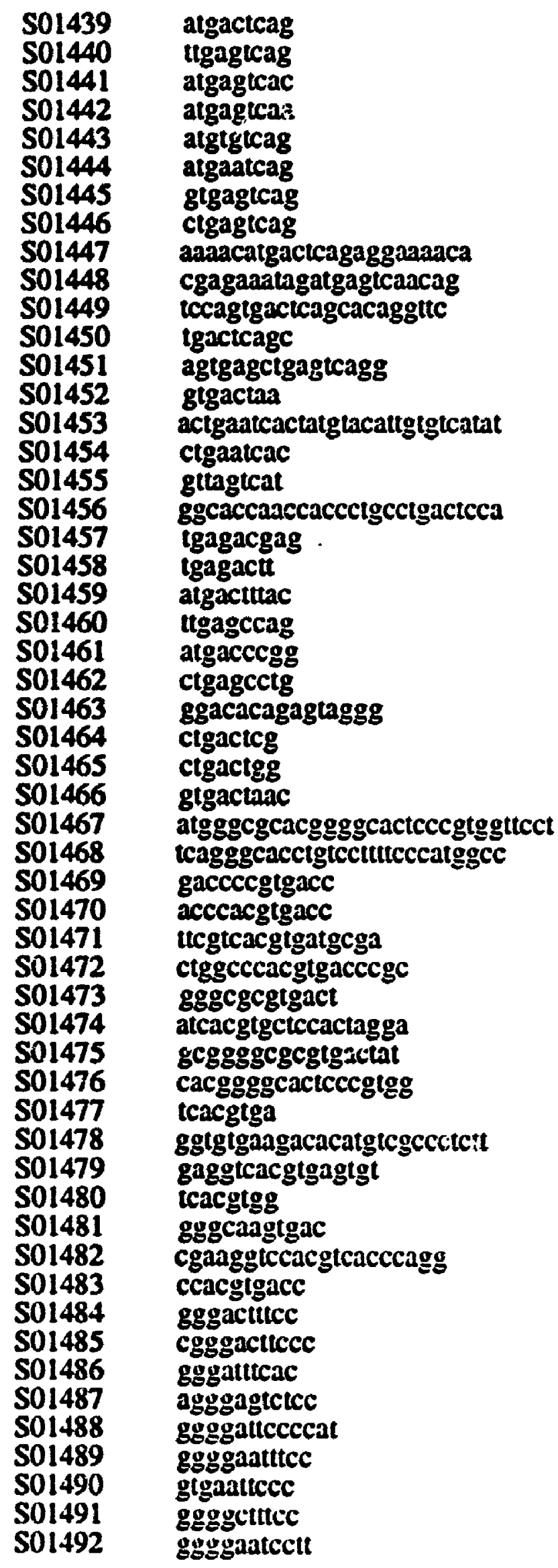




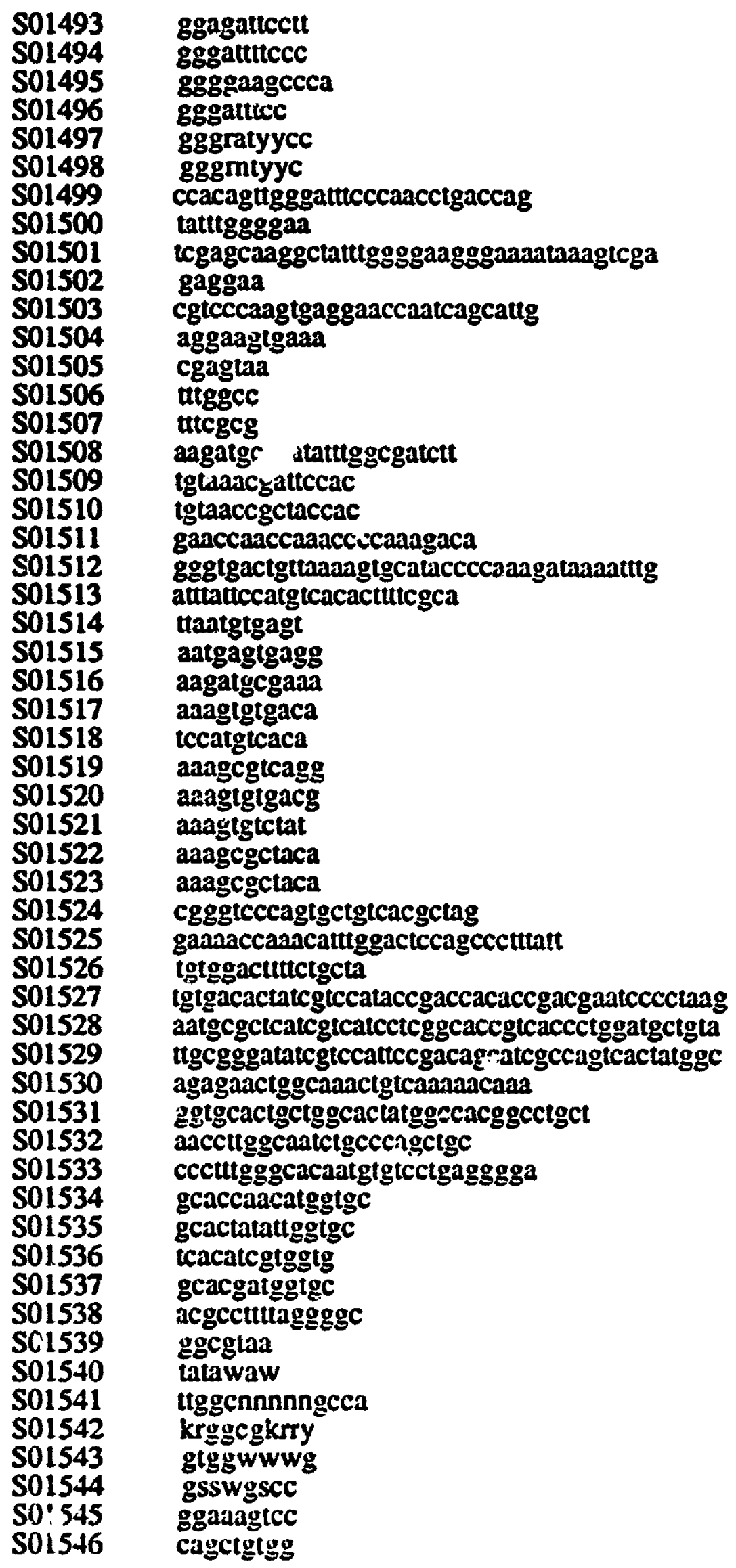




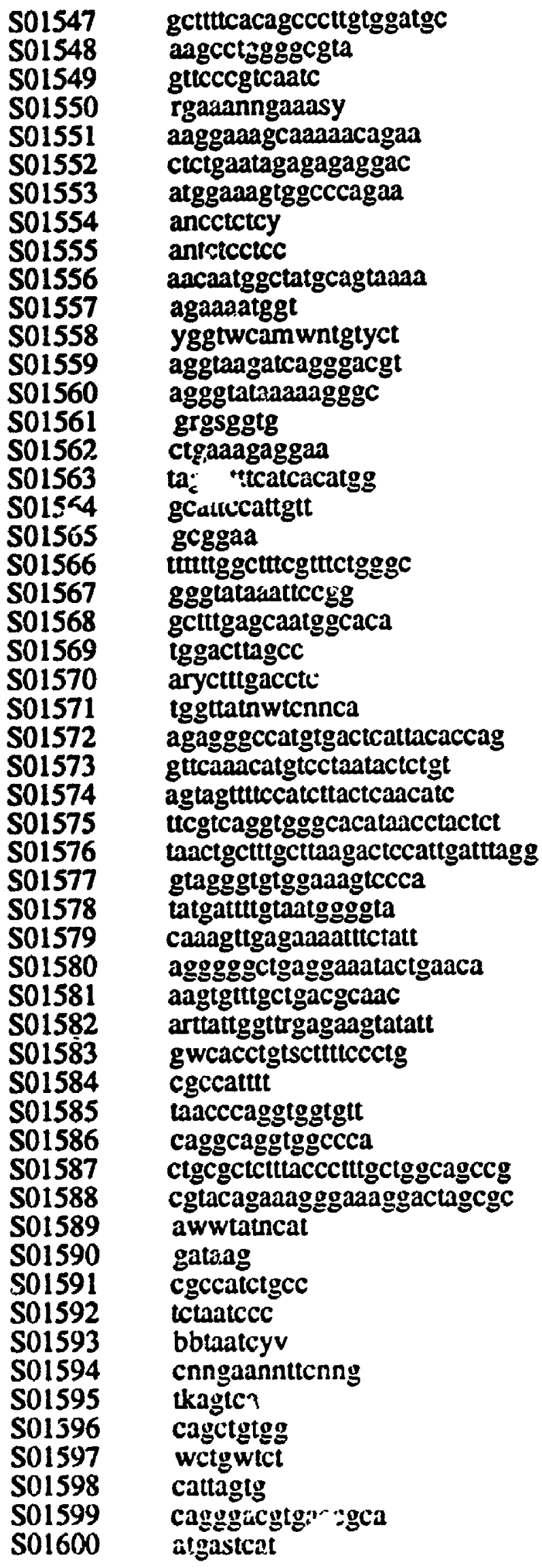




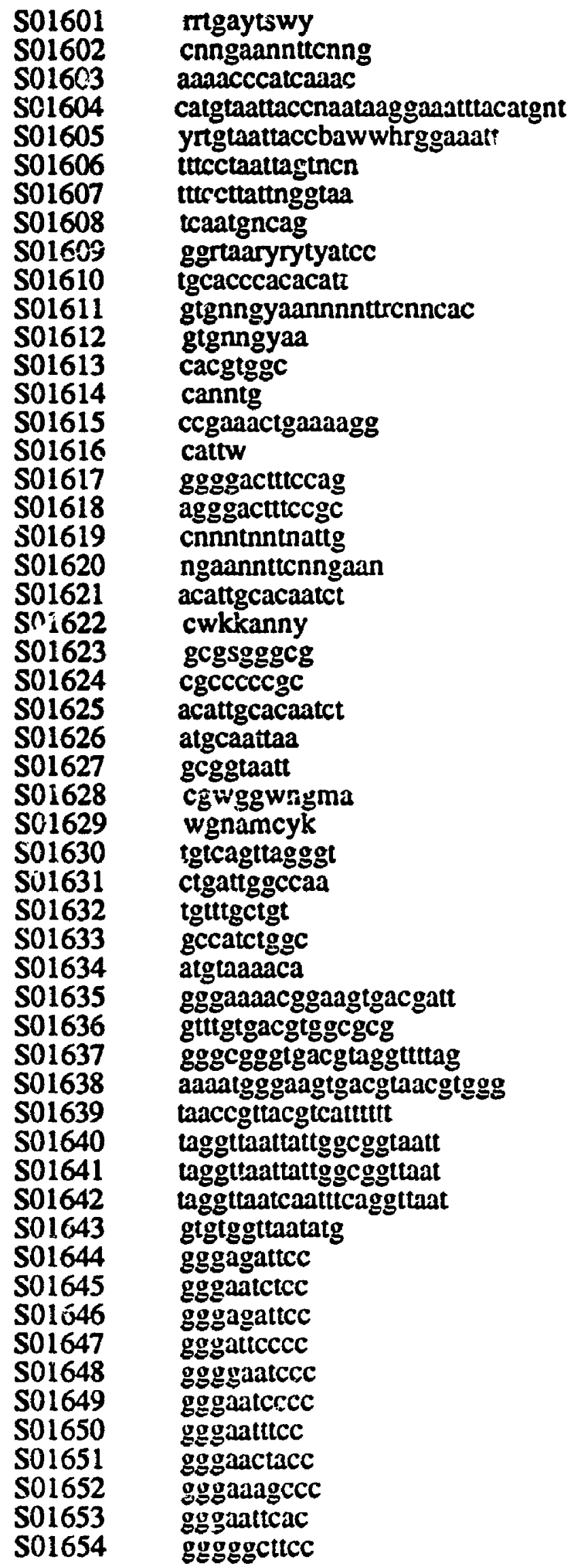




\begin{tabular}{|c|c|}
\hline 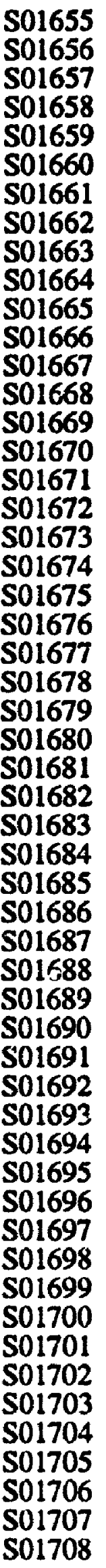 & 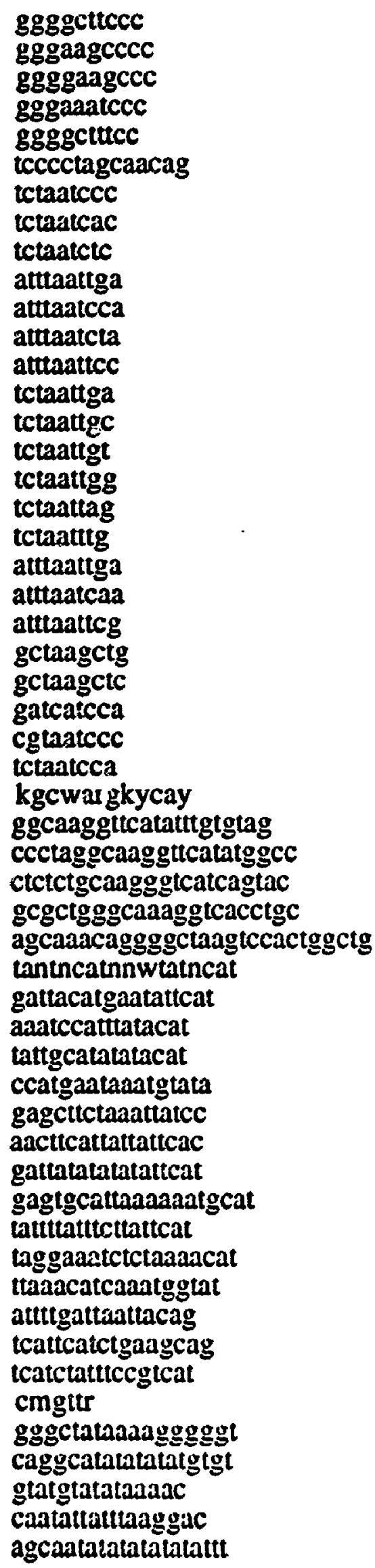 \\
\hline
\end{tabular}




\begin{tabular}{|c|c|}
\hline $\begin{array}{l}\text { S01709 } \\
\text { S01710 } \\
\text { S01711 } \\
\text { S01712 } \\
\text { S01713 } \\
\text { S01714 } \\
\text { S01715 } \\
\text { S01716 } \\
\text { S01717 } \\
\text { S01718 } \\
\text { S01719 } \\
\text { S01720 } \\
\text { S01721 } \\
\text { S01722 } \\
\text { S01723 } \\
\text { S01724 } \\
\text { S01725 } \\
\text { S01726 } \\
\text { S01727 } \\
\text { S01728 } \\
\text { S01729 } \\
\text { S01730 } \\
\text { S01731 } \\
\text { S01732 } \\
\text { S01733 } \\
\text { S01734 } \\
\text { S01735 } \\
\text { S01736 } \\
\text { S01737 } \\
\text { S01738 } \\
\text { S01739 } \\
\text { S01740 } \\
\text { S01741 } \\
\text { S01742 } \\
\text { S01743 } \\
\text { S01744 } \\
\text { S01745 } \\
\text { S01746 } \\
\text { S01747 } \\
\text { S01748 } \\
\text { S01749 } \\
\text { S01750 } \\
\text { S01751 } \\
\text { S01752 } \\
\text { S01753 } \\
\text { S01754 } \\
\text { S01755 } \\
\text { S01756 } \\
\text { S01757 } \\
\text { S01758 } \\
\text { S01759 } \\
\text { S0:760 } \\
\text { S01761 } \\
\text { S01762 }\end{array}$ & 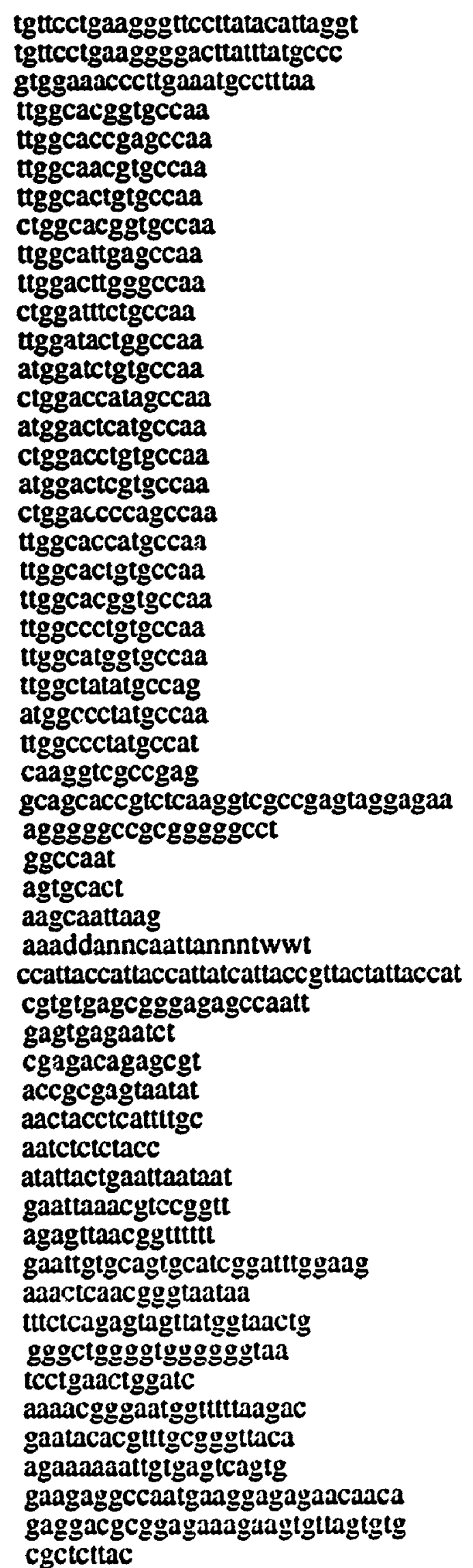 \\
\hline
\end{tabular}




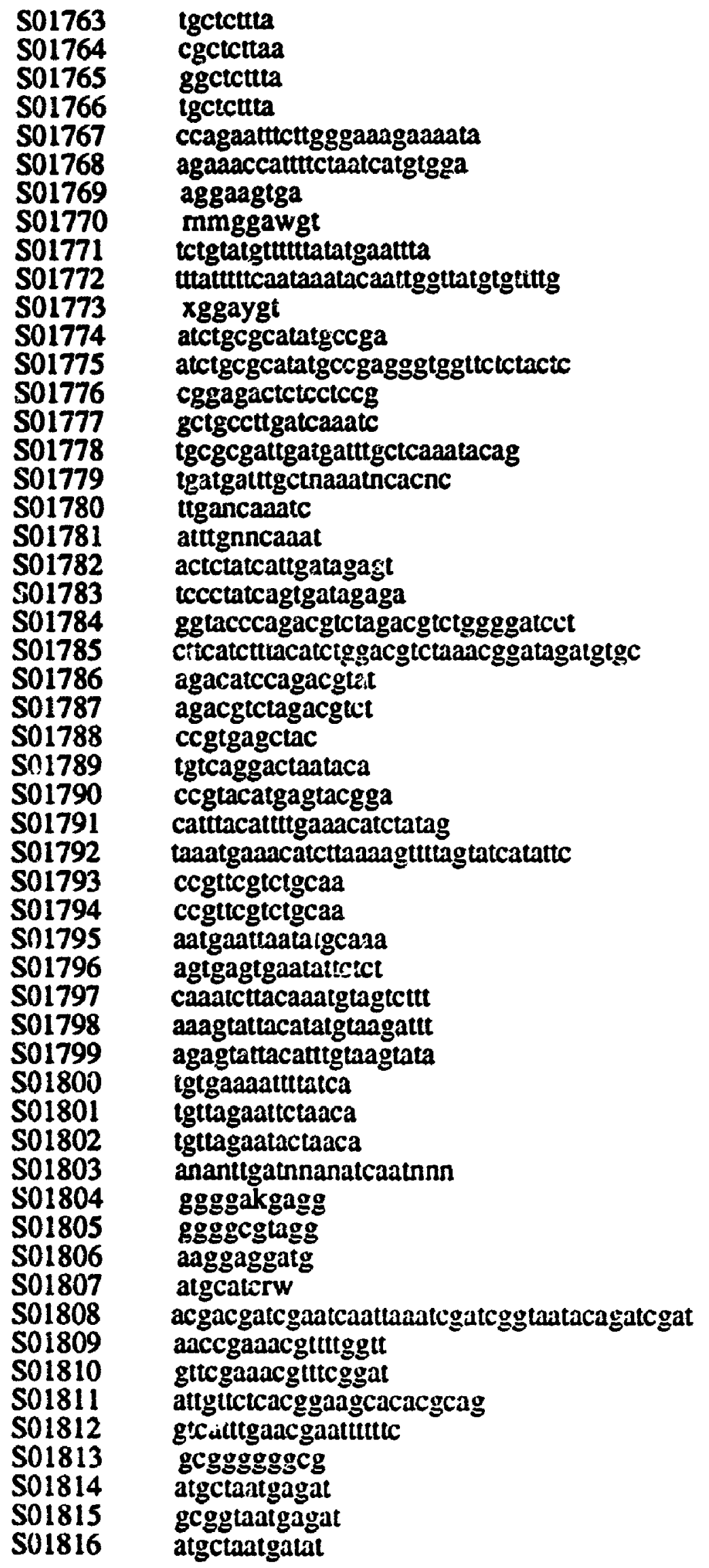




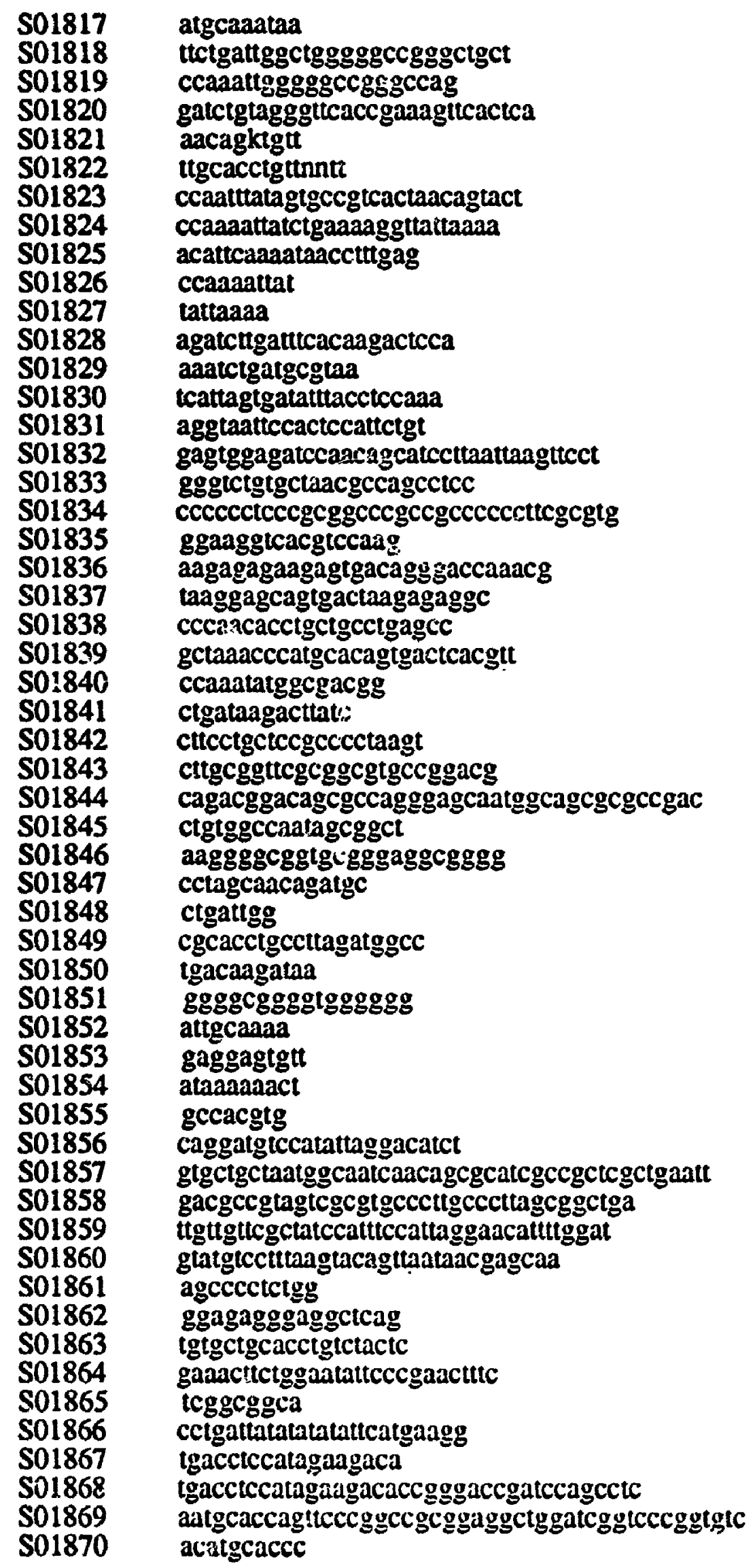




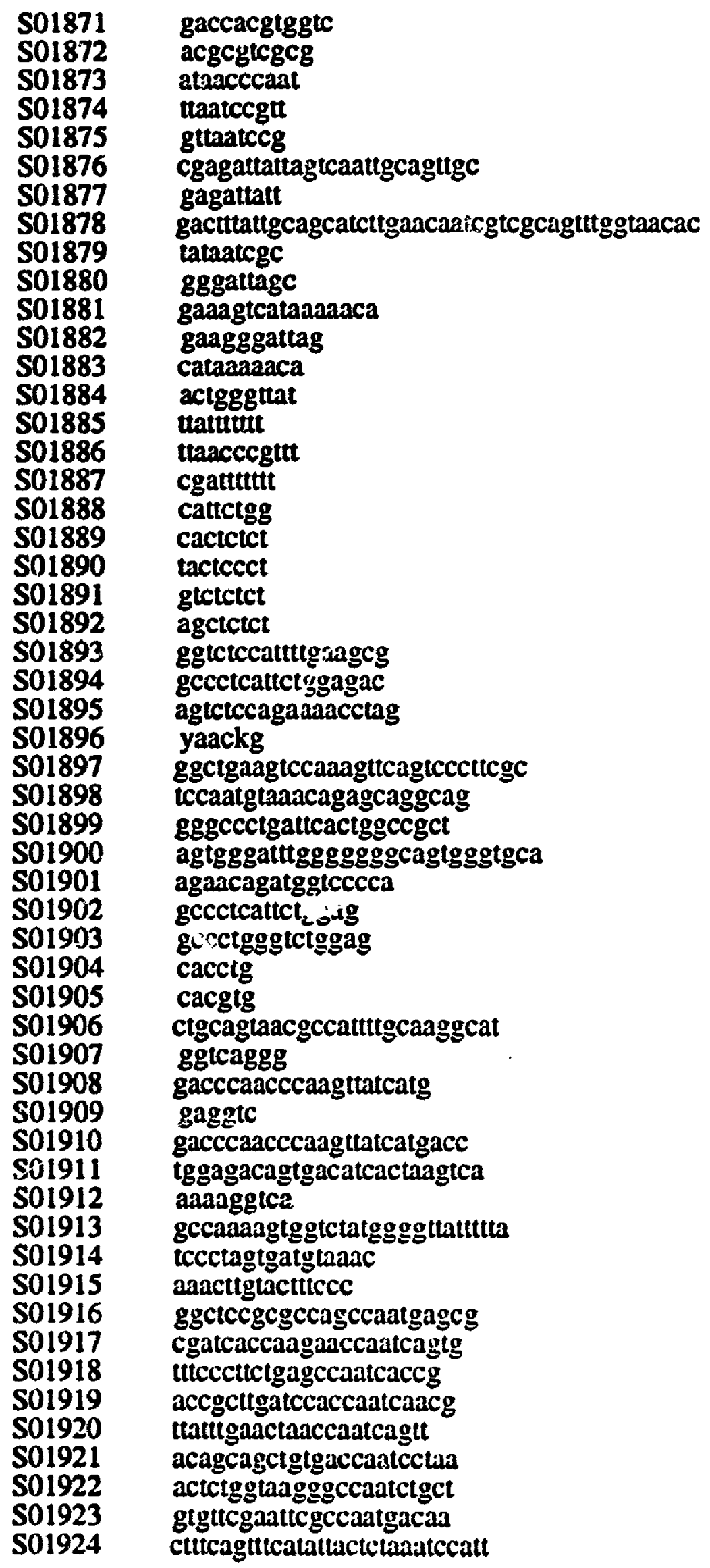




\begin{tabular}{|c|c|}
\hline 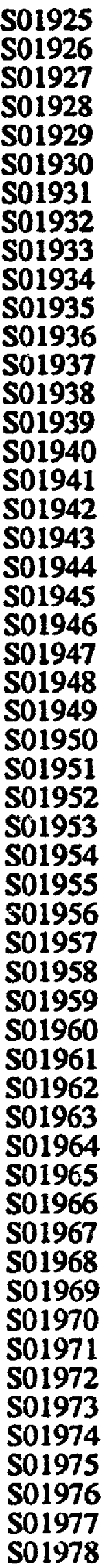 & 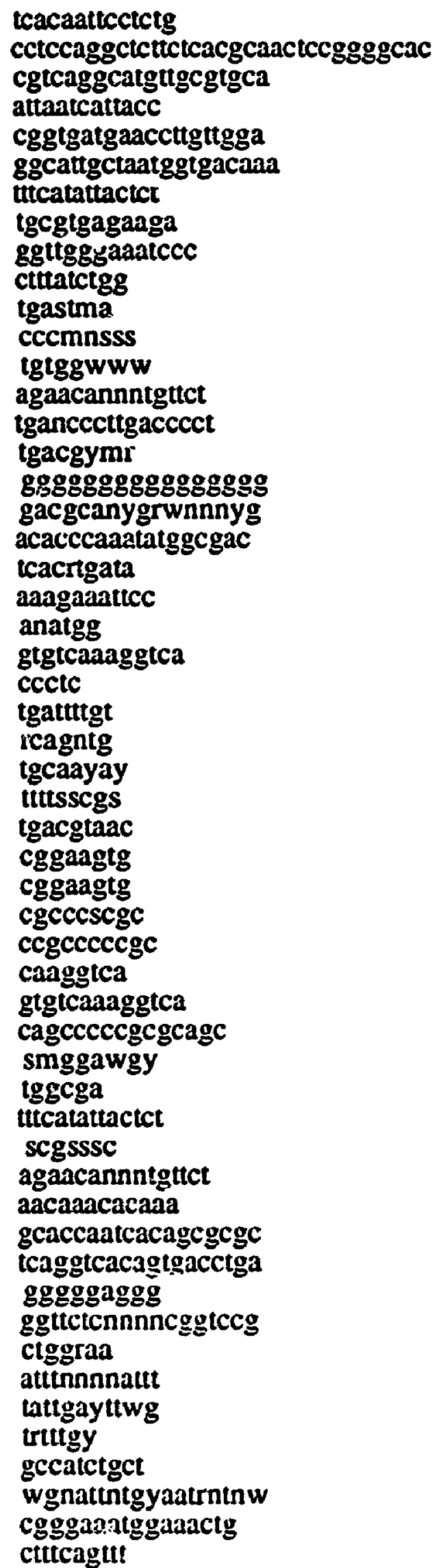 \\
\hline
\end{tabular}




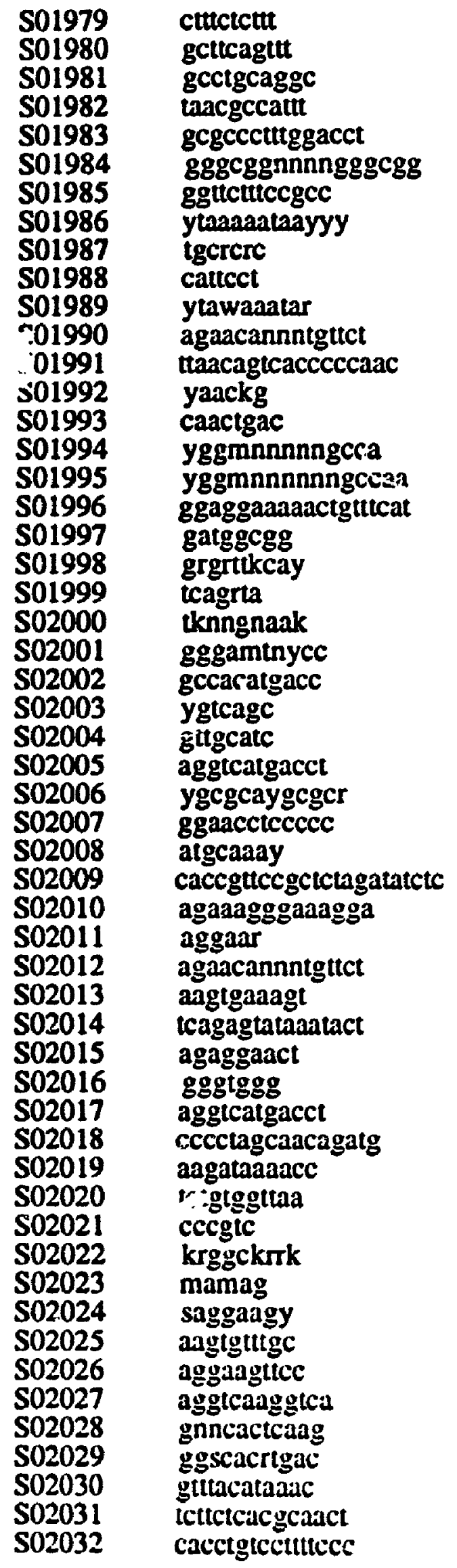




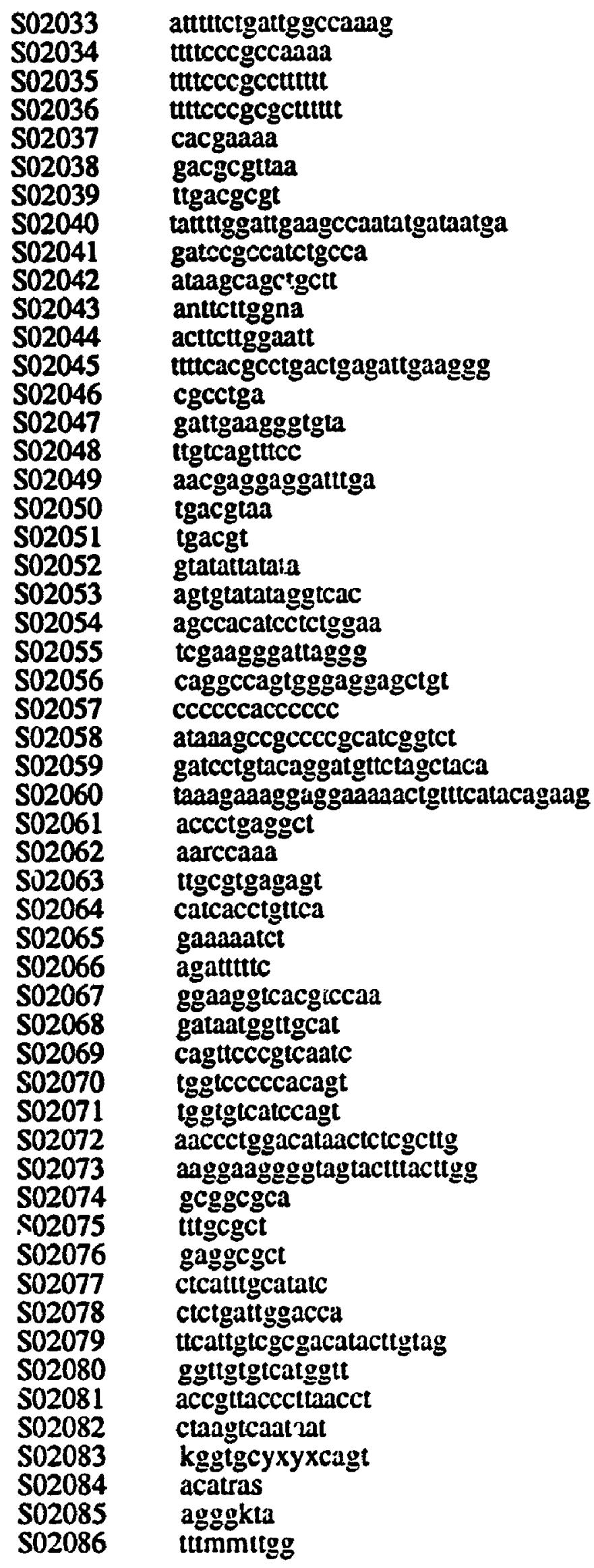




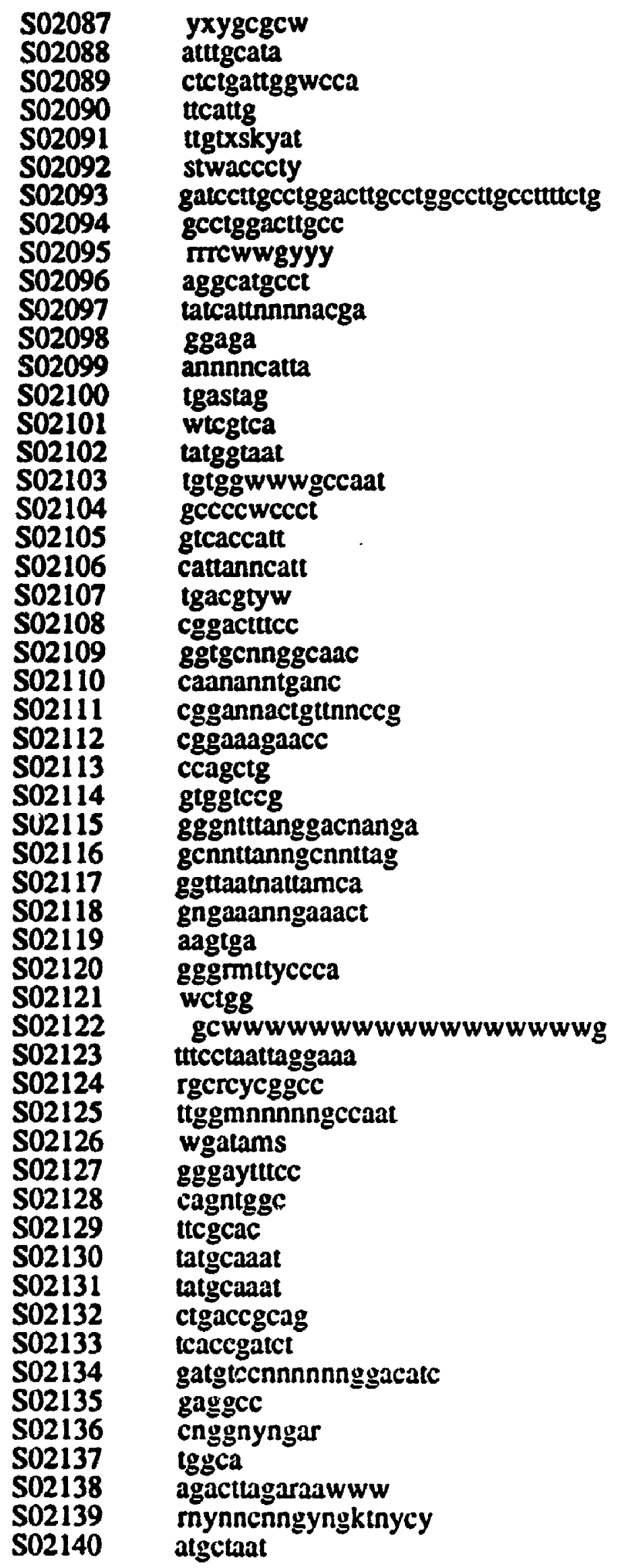




\begin{tabular}{|c|c|}
\hline 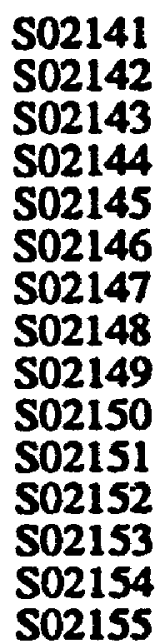 & $\begin{array}{l}\text { acaycygtrcat } \\
\text { tstcacgcwas } \\
\text { ggaggctgaggca } \\
\text { ctctct! } \\
\text { tgcgcgctctc } \\
\text { tctctgccg } \\
\text { gtctcagtgca } \\
\text { gaacatgtctaagcatgctg } \\
\text { gagcgtgtctaagctcgtgg } \\
\text { ccctggcacattcgtgcaa } \\
\text { ctictgcgtcagcgccagtat } \\
\text { aagctagtaccagttgagccag } \\
\text { gacaggaacag } \\
\text { ggcaggaagta } \\
\text { cctcttcttccagaggatgtggcttctgcga }\end{array}$ \\
\hline
\end{tabular}




\section{Appendix C}

\section{Human Interferon Transcription Factors}

The Human Interferon Transcription Factors Database (HulFNTFD) is a comprehensive list of known human interferon transcription factor and promoter element nucleotide recognition sequences. HuIFNTFD human interferon transcription factor and promoter element nucleotide recognition sequences are presented.

Enzyme Recognition Sequence

$\begin{array}{ll}\text { IFN001 } & \text { aaaaacttgtgaaaatacgtaatcc } \\ \text { IFN002 } & \text { aaaactgaaa } \\ \text { IFN003 } & \text { aaaatgtaaa } \\ \text { IFN004 } & \text { aaagaaar: } \\ \text { IFN005 } & \text { aaagga } \\ \text { IFN006 } & \text { aaatga } \\ \text { IFN007 } & \text { aaatga } \\ \text { IFN008 } & \text { aaatgactgaatatcgacttgct } \\ \text { IFN009 } & \text { aaatgg } \\ \text { IFN010 } & \text { aaatgt } \\ \text { IFN011 } & \text { aaattcctctgaatagagagaggac } \\ \text { IFN012 } & \text { aacaga } \\ \text { IFN013 } & \text { aactga } \\ \text { IFN014 } & \text { aagaaag } \\ \text { IFN015 } & \text { aagaaag } \\ \text { IFN016 } & \text { aagaaag } \\ \text { IFN017 } & \text { aagga: } \\ \text { IFN018 } & \text { aaggaaag } \\ \text { IFN019 } & \text { aaggaaag } \\ \text { IFN020 } & \text { aaggaaag } \\ \text { IFN021 } & \text { aaggaaagcaaaacagaa } \\ \text { IFN022 } & \text { aaggga } \\ \text { IFN023 } & \text { aaggga } \\ \text { IFN024 } & \text { aagtga } \\ \text { IFN025 } & \text { aagtga } \\ \text { IFN026 } & \text { aagtgaatttttgagttctttt } \\ \text { IFN027 } & \text { aagtgg } \\ \text { IFN028 } & \text { aagtgg } \\ \text { IFN029 } & \text { aartgraa } \\ \text { IFN030 } & \text { aatgaaal } \\ \text { IFN031 } & \text { aatgtt } \\ \text { IFN032 } & \text { aattcctctga } \\ & \end{array}$

\section{Reference}

Penix et. al. (1993)

Goodbourn et. al. (1986)

Goodbourn et. al. (1986)

Ryals et. al. (1985)

Harada et. al. (1989)

Harada et. al. (:989)

Miyamoto et. al. (1988)

Hardy et. al. (198.5)

Miyamoto et. al. (1988)

Miyamoto et. al. (1988)

Hiscott et. al. (1989)

Maniatis (1988)

Miyamoto et. al. (1988)

Ryals et. al. (1985)

Ryals et. al. (1985)

Ryals et. al. (1985)

Maniatis (1988)

Ryals et. al. (1985)

Ryals et. al. (1985)

Ryals et. al. (1985)

Ryals et. al. (1985)

Harada et. al. (1989)

Miyamoto et. al. (1988)

Harada et. al. (1989)

Miyamoto et. al. (1988)

Hardy et. al. (1985)

Miyamoto et. al. (1988)

Miyamoto et. al. (1988)

Taniguchi (1989)

Ryals et. al. (1985)

Maniatis (1988)

Nourbakhsh et. al. (1993) 


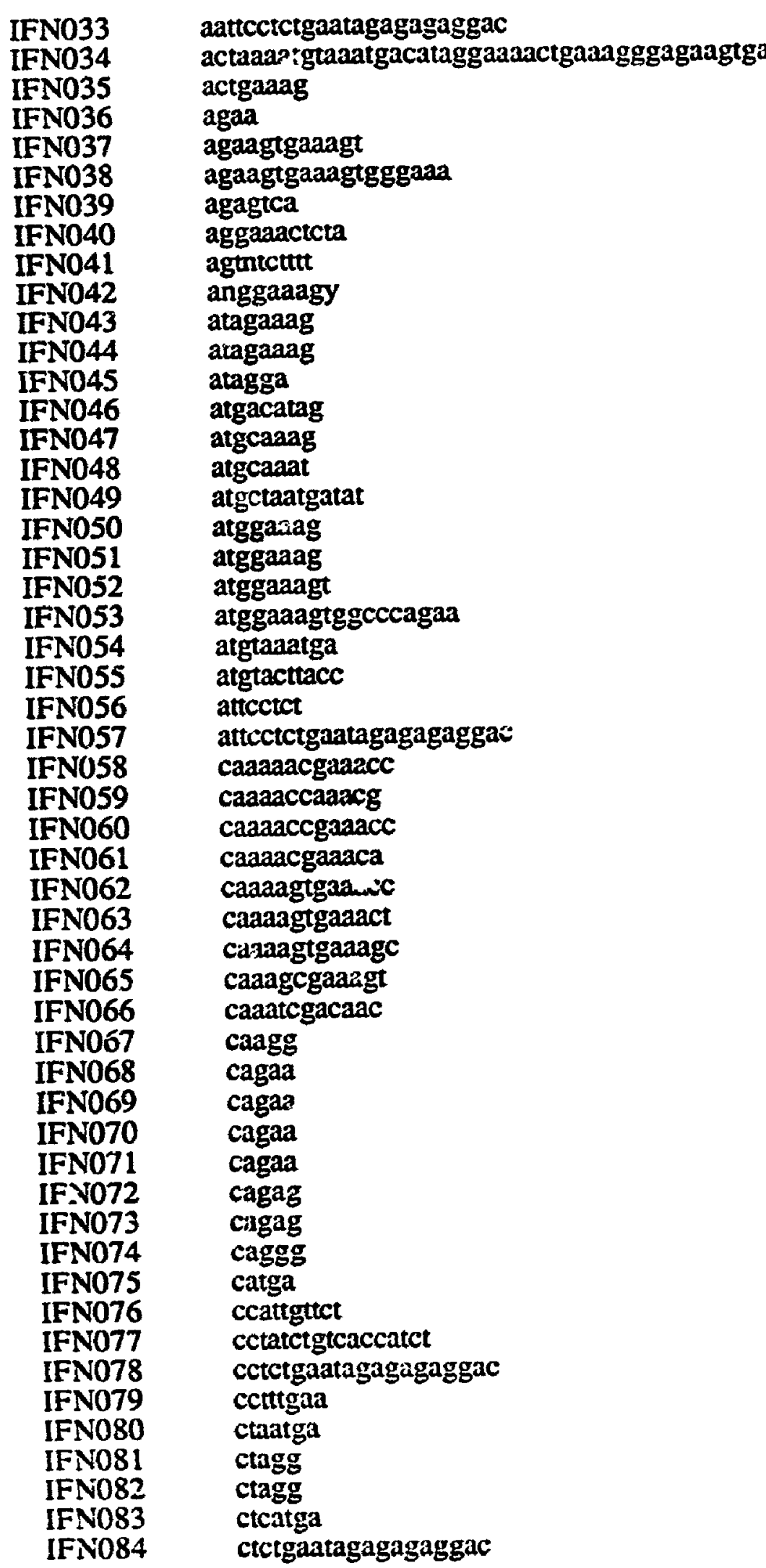

Hiscott et. al. (1989)

Dinter et. al. (1983)

Ryals et. al. (1985)

Ryals et. al. (1985)

Du et. al. (1992)

Maniatis (1988)

Schwarz et. al. (1993)

Fraser et. al. (1991)

Hardy et. al. (1987)

Kuhl et. al. (1987)

Ryals et. al. (1985)

Ryals et. al. (1985)

Miyamoto ct. all. (1988)

Du et. al. (1992)

Garcia-Blanco et. al. (1989)

Garcia-Blanco et. al. (1989)

Garcia-Blanco et. al. (1989)

Ryals et. al. (1985)

Ryals et. al. (1985)

Kuhl et. al. (1987)

Ryals et. al. (1985)

Tanaka et. al. (1992)

Garcia-Blanco et. al (1989)

Wang et. al. (1990)

Hiscott et. al. (1989)

Tanaka et. al. (1993)

Tanaka et. al. (1993)

Tanaka et. al. (1993)

Tanaka et. al. (1993)

Tanaka et. al. (1993)

Taraka et. al. (1993)

Tanaka et. al. (1993)

Tanaka et. al. (1993)

Tanaka et. al. (1993)

Ryals et. al. (1985)

Ryals et. al. (1985)

Ryals et. al. (1985)

Ryals et. al. (1985)

Ryals et. al. (1985)

Ryals et. al. (1985)

Ryals et. al. (1985)

Ryals et. al. (1985)

Ryals et. al. (1985)

Giese et. al. (1992)

Penix et. al. (1993)

Hiscott et. al. (1989)

Giese et. al. (1992)

Garcia-Blanco et. al. (1989)

Ryals et. al. (1985.

Ryals et. al. (1985)

Garcia-Blanco et. al. (1989)!

Hiscott et. al. (1980)! 


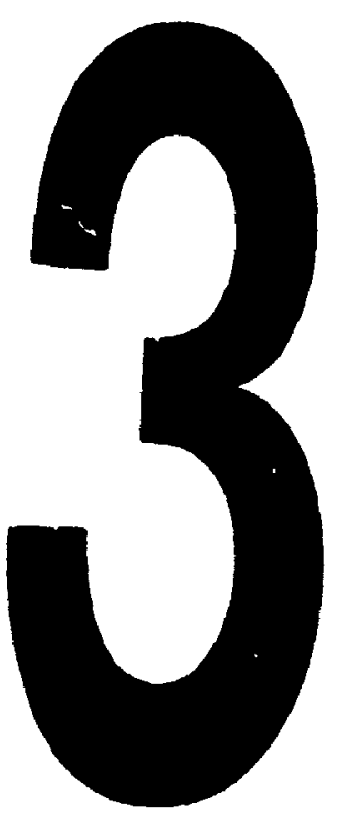

PM-1 3\%" $\times 4$ " PHOTOGRAPHIC MICROCOPY TARGET MBS 1010a ANSI/ISO \#2 EQUIVALENT

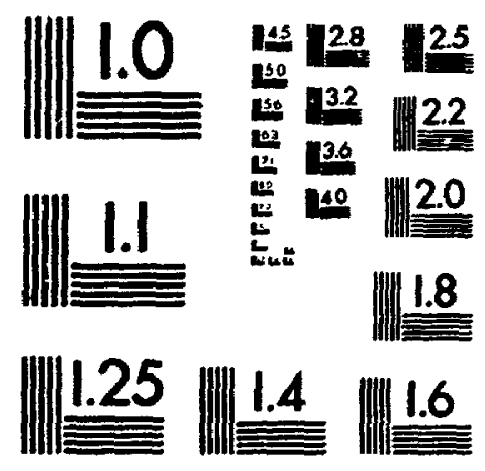

PRECISIONSW RESOLUTION TARGETS 


\begin{tabular}{|c|c|c|}
\hline IFN085 & ga & Ryals et. al. (1985) \\
\hline IFN086 & gaaa & Harada et. al. (1989) \\
\hline IFN087 & gaaaacgaaacc & Tanaka et. al. (1993) \\
\hline IFN088 & gaaaacgaaagt & Tanaka et. al. (1993) \\
\hline IFN089 & gaaaactgaaagg & Tanaka et. al. (1993) \\
\hline IFN090 & gaaaactgaaagg & Du et. al. (1992) \\
\hline IFN091 & gaaaagcgaaagc & Tanaka et. al. (1993) \\
\hline IFN092 & gaaaagcgaaagc & Truaka et. al. (1993) \\
\hline IFN093 & gaaace & MacDonald et. al. (1990) \\
\hline IFN094 & gaaacg & MacDonald et. al. (1990) \\
\hline IFN095 & gaaact & MacDonald et. al. (1990) \\
\hline IFN096 & gaaactgaaacc & Tanaka et. al. (1993) \\
\hline IFN097 & gaaactgaaacc & Tanaka et. al. (1993) \\
\hline IFN098 & gaaage & MacDonald et. al. (1990) \\
\hline IFN099 & gaaagcaaaagt & Taııka et. al. (1993) \\
\hline IFN100 & gaaagcgaaacc & Tanaka et. al. (1993) \\
\hline IFN101 & gaaagcgaaacc & Tanaka et. al. (1993) \\
\hline IFN102 & gaaagcgaaagt & Tanaka et. al. (1993) \\
\hline IFN103 & gaaagcgaaagt & Tanaka et. al. (1993) \\
\hline IFN104 & gaaagt & MacDonald et. al. (199r \\
\hline $1 \because N=05$ & gaaagtgaaacc & Tanaka et. al. (1993) \\
\hline IFNl06 & gaaagtgaaacc & Tanaka et. al. (1993) \\
\hline IFN107 & gaaagtgaaacc & Tanaka et. al. (1993) \\
\hline IFN108 & gaaagtgaaacc & Tanaka et. al. (1993) \\
\hline IFN109 & gaaagtgaaacc & Tanaka et. al. (1993) \\
\hline IFI. 1110 & gaaagtgaaacc & Tanaka ei. al. (1993) \\
\hline IFN111 & gaaagtgaaacc & Tanaka :t. al. (1993) \\
\hline IFN112 & gaaagtgaaacg & Tanaka et. al. (1993) \\
\hline IFN113 & gaaagtgaaact & Tanaka et. al. (1993) \\
\hline IFN114 & gaaagtgaaact & Tanaka et. al. (1993) \\
\hline IFN115 & gaaagtgaaagt & Tanaka et. al. (1993) \\
\hline IFN116 & gaaagtgaaagt & Tanaka et. al. (1993) \\
\hline IFN117 & gaaann & MacDonald et. al. (1990) \\
\hline IFN118 & gaaatg & MacDonald et. al. (1990) \\
\hline IFN119 & gaaatgaaagt & Tanaka et. al. (1993) \\
\hline IFN & gaaatggaaa & MacDonald et. al. (1990) \\
\hline IFN & gaaatggaaag & Tanaka et. al. (1992) \\
\hline IFN & gaaatggaaagt & MacDonald et. al. (1990) \\
\hline IFN & gaaattcctctgaatagagagaggar & Hiscott et. al. (1989) \\
\hline IFN & gaagtgaaag & Tanaka et. al. (1992) \\
\hline IFN & gaartgraa & Taniguchi (1989) \\
\hline IFN & gagaa & Ryals et. al. (1985) \\
\hline IFI & gagaa & Ryals et. al. (1985) \\
\hline IF] & gagaa & Ryals et. al. (1985) \\
\hline IFI & gagaa & Ryals et. al. (1985) \\
\hline IF & gagaagtgaaagt & Tanaka et. al. (1993) \\
\hline IF & gagaagtgaaag: & Tanaka et. al. (1992) \\
\hline IFI & gagaagtgaaagtg & Maniatis et. al. (1992) \\
\hline IFI & gagaagtgiaagtgggaaatt? & Kuhl et al. (1987) \\
\hline IFI & gagaagtgaaagtgggaaattcctctgaatagagagaggac & Goodbourn et. al. (1985) \\
\hline IFI & gagtgcatgaaggauagcaaanacagaaatggaaagtggcc & cagaa Ryals et. al. (1985) \\
\hline & gataacgaaago & Tanaka et. al. (1993) \\
\hline IF & gatagtganacc & Tanaka et. al. (1993) \\
\hline IFI & gcttgtt & Giese et. al. (1992) \\
\hline
\end{tabular}




\begin{tabular}{|c|c|}
\hline IFN139 & ggaaaactgaaagggagaagtgaaagtggg \\
\hline [FN]40 & ggaaattcctctgaatagagagaggac \\
\hline IFN141 & gggaazattcc \\
\hline IFN1 42 & gggaatctcc \\
\hline IFN143 & gggaattcce \\
\hline IFN144 & gggactttcc \\
\hline IFN145 & gggattttccc \\
\hline IFN146 & ggggaatcccc \\
\hline IFN147 & ggggactttccac \\
\hline IFN148 & ggggattcccc \\
\hline IFN149 & gggrhwyymy \\
\hline IFN150 & gtagaaaa \\
\hline IFN151 & gtagaaag \\
\hline IFN152 & gtagaaag \\
\hline IFN153 & gtgaaag \\
\hline IFN154 & gtgacgtmr \\
\hline IFN155 & gtgagtma \\
\hline IFN156 & gtgggaaattcc \\
\hline IFN157 & taaaaccgaaagc \\
\hline IFN158 & taataggtgtaata \\
\hline IFN159 & taggasaa \\
\hline IFN160 & tatgataatga \\
\hline IFN161 & tcaattaaatga \\
\hline IFN162 & tcctctgaatagagagaggac \\
\hline IFN163 & tctgaatagagagaggac \\
\hline IFN164 & tgaaagtgaaagt \\
\hline IFN165 & tgacgtmr \\
\hline IFN166 & tgtaaatgacatag \\
\hline IFN167 & ttcctctgaatagagagaggac \\
\hline IFN168 & ttctttgtty \\
\hline IFN169 & ttctttaaaattt \\
\hline IFN170 & tttatgaattttccaaaa \\
\hline IFN171 & yyattgttctc \\
\hline
\end{tabular}

Dron et. al. (1992)

Hiscott et. al. (1989)

Baeuerle (1991)

Ballard et. al. (1990)

Ballard et. al. (1990)

Baeuerle (1991)

Nakayama et. al. (1992)

Fan et. al. (1990)

Nakayama et. al. (1992)

Nakayama et. al. (1992)

Lenardo et. al. (1989)

Ryals et. al. (1985)

Ryals et. al. (1985)

Ryals et. al. (1985)

Ryals et. al. (1985)

Hai et. al. (1989)

Hai et. al. (1989)

Maniatis et. al. (1592)

Tanaka et. al. (1993)

Garcia-Blanco et. al. (1989)

Ryals et. al. (1985)

Garcia-Blanco et. 2.. (1989)

Garcia-Blanco et. 2.1. (1989)

Hiscott et. al. (1989)

Hiscott et. al. (1989)

Naf et. al. (1991)

Du et. al. (1992)

Maniatis et. al. (1992)

Hiscott et. al. (1989)

Giese et. al. (1992)

Hardy et. al. (1985)

Sica et. al. (1992)

Giese et. al. (1992) 
Appendix D

\section{Human Interferon Alpha-1 Gene}

\section{D.1 Nucleotide Sequence}

The genomic DNA sequence of the human interferon-alpha 1 gene was retrieved from the Genbank sequence file humifnad (Mantei et. al., 1980) (Nagata et. al., 1980)

(Goeddel et. al., 1981) (Bowden et. al., 1984) (Benson et. al., 1993).

1 aaaacaaaac attigagaaa cacggctcta aactcatgta aagagtgcat gaaggaaagc

61 aaaaacagaa atggaaagtg geccagaage attaagaaag tggaaatcag tatgttccct

121 attraaggca tttgcags :a gcaaggcctt cagagaacct agagcccaag gttcagagtc

181 acccatctca gcaagcccag aagtatctgc aatatctacg atggcctcge ccttgettt

241 actgatggtc ctggtggtge tcagctgcaa gtcaagctgc tcrctgggct gtgatctcce

301 tgagacccac agcctggata acaggaggac cttgatgetc ctggcacaaa tgagcagaat

361 ctctccttcc tcctgtctga tggacagaca tgacttgga tttccceagg aggagtttga

421 tggcaaccag ttccagaagg ctccagccat ctctgtcctc catgagctga tccagcagat

481 cttcaacctc tttaccacaa aagattcatc tgctgcttgg gatgaggacc tcctagacaa

541 attctgcace gaactctacc agcagctgaa tgacttggaa gectgtgtga tgcaggagga

601 gagggtggga gaaactccce tgatgaatge ggactccatc ttggctgtga agaaatactt

661 ccgaagaatc actctctatc tgacagagaa gaaatacagc ccttgtgect gggaggttgt

721 cagagcagaa atcatgagat ccctctcttt atcaacaaac ttgcaagaaa gattaaggag

781 gaaggaataa catctggtcc aacatgaaaa caattcttat tgactcatac accaggtcac

841 gcttrcatga attctgtcar ttcaaagact ctcasccctg ctataactat gaccatgetg

901 ataaactgat ttatctatt aaatatttat ttaactattc ataagattta aattatttt

961 gttcatataa cgtcatgtgc acctttacac tgtggttagt gtaataaaac atgttcctta

1021 tattractca atccattatt ttgtgttgtt cattaaactt ttactatagg aacttcctgt

1081 atgtgttcat tcttraatat ganattccta gectgactgt gcaacctgat tagagaataa

1141 agggtatatt ttatttgctt atcattatta tatgtaaga 


\section{D.2 Restriction Endonuclease Sites}

The human interferon-alpha 1 gene restriction endonuclease recognition sites are arranged by ascending sequence location.

\begin{tabular}{|c|c|}
\hline Name & Site \\
\hline BcefI & 22 \\
\hline CVIJI & 24 \\
\hline NlaIII & 35 \\
\hline CVIRI & 46 \\
\hline NlaIII & 48 \\
\hline HaeIII & 80 \\
\hline AsuI & 80 \\
\hline CVIJI & 80 \\
\hline Mse I & 92 \\
\hline$E \operatorname{coN} I$ & 118 \\
\hline BSIYI & 118 \\
\hline MseI & 123 \\
\hline CVIRI & 133 \\
\hline StuI & 144 \\
\hline Hae I & 144 \\
\hline HaeIII & 145 \\
\hline CVIJI & 145 \\
\hline MaeI & 159 \\
\hline HgiJII & 162 \\
\hline Sdu I & 162 \\
\hline CVIJI & 163 \\
\hline StyI & 166 \\
\hline SecI & \\
\hline $\begin{array}{l}\text { PleI } \\
\text { HinfI }\end{array}$ & $\begin{array}{l}176 \\
176\end{array}$ \\
\hline $\operatorname{Isp} 45 \mathrm{I}$ & 178 \\
\hline MaeIII & 178 \\
\hline TaqIIa & 180 \\
\hline BccI & 183 \\
\hline $\begin{array}{l}\text { DdeI } \\
\operatorname{Cac} 8 I\end{array}$ & $\begin{array}{l}187 \\
191\end{array}$ \\
\hline Cuj.JI & 19 \\
\hline CVIRI & 208 \\
\hline Hae I & 222 \\
\hline HaeIII & 223 \\
\hline CviJI & 223 \\
\hline MnII & 225 \\
\hline AvaII & 247 \\
\hline AsuI & 247 \\
\hline EcoRII & 25 \\
\hline ScrEI & 25 \\
\hline HgIAI & \\
\hline
\end{tabular}




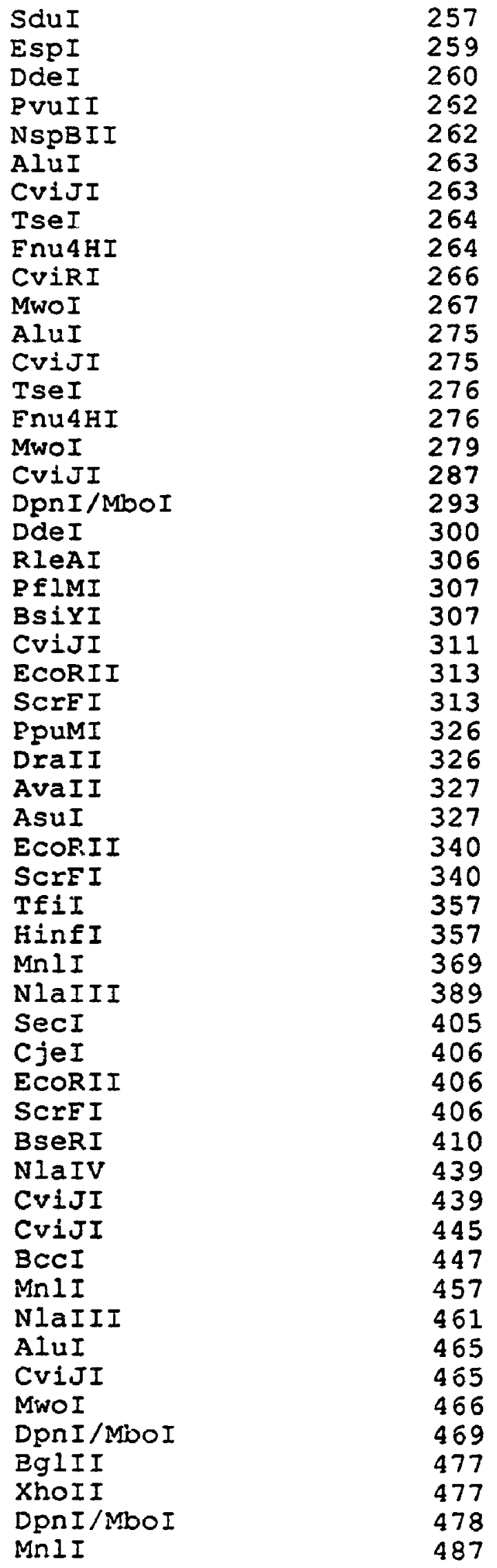




$\begin{array}{ll}\text { TfII } & 503 \\ \text { HinfI } & 503 \\ \text { TseI } & 512 \\ \text { EnuAHI } & 512 \\ \text { FokI } & 520 \\ \text { PpuMI } & 525 \\ \text { DraII } & 525 \\ \text { AvaII } & 526 \\ \text { AsuI } & 526 \\ \text { MnII } & 529 \\ \text { MaeI } & 533 \\ \text { ApoI } & 539 \\ \text { TspEI } & 540 \\ \text { CviRI } & 545 \\ \text { AIwNI } & 560 \\ \text { BbvI } & 562 \\ \text { TseI } & 562 \\ \text { FnuAHI } & 562 \\ \text { PvuII } & 563 \\ \text { NspBII } & 563 \\ \text { AluI } & 564 \\ \text { CviJI } & 532 \\ \text { CvIJI } & \end{array}$




$\begin{array}{lr}\text { ScIEI } & 832 \\ \text { Tsp45I } & 836 \\ \text { MaeIII } & 836 \\ \text { BspHI } & 845 \\ \text { NlaIII } & 846 \\ \text { EcoRI } & 849 \\ \text { ApoI } & 849 \\ \text { TspEI } & 850 \\ \text { HinfI } & 867 \\ \text { NlaIII } & 894 \\ \text { SwaI } & 917 \\ \text { AhaIII } & 918 \\ \text { MseI } & 919 \\ \text { SspI } & 922 \\ \text { MseI } & 931 \\ \text { SwaI } & 946 \\ \text { AhaIII } & 947 \\ \text { MseI } & 948 \\ \text { TspEI } & 951 \\ \text { MaeII } & 970 \\ \text { NlaIII } & 974 \\ \text { ApaII } & 977 \\ \text { HgiAI } & 977 \\ \text { SduI } & 977 \\ \text { CviRI } & 978 \\ \text { BsplUIII } & 1009 \\ \text { AfIIII } & 1009 \\ \text { NspI } & 1009 \\ \text { NlaIII } & 1010 \\ \text { MseI } & 1053 \\ \text { SfeI } & 1064 \\ \text { MseI } & 1094 \\ \text { ApoI } & 1102 \\ \text { TspEI } & 1103 \\ \text { MaeI } & 1108 \\ \text { CviJI } & 1110 \\ \text { CviRI } & 1120\end{array}$

The human interferon-alpha 1 gene restriction endonuclease recognition sites are arranged by enzyme.

Name

AfIIII

AhaIII

AhaIII

AluI

AluI

AluI

AIUI site

1009

918

947

263

275

465

554 


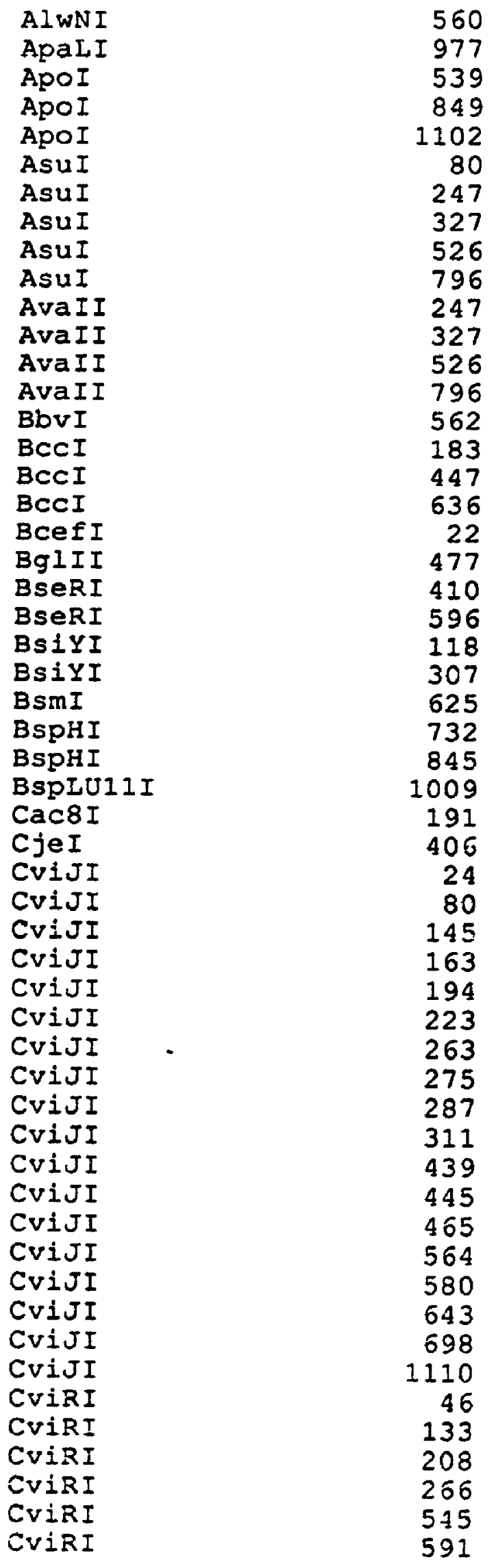




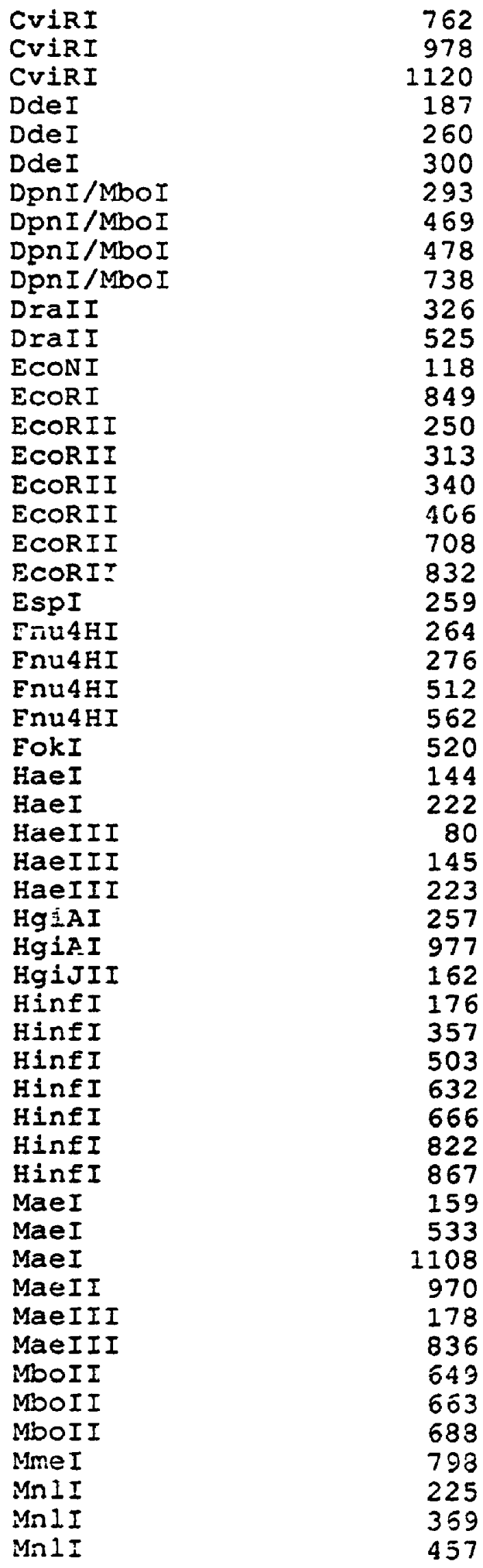




$\begin{array}{lr}\text { MnII } & 487 \\ \text { MnII } & 529 \\ \text { MnII } & 742 \\ \text { MseI } & 92 \\ \text { MseI } & 123 \\ \text { MseI } & 773 \\ \text { MseI } & 919 \\ \text { MseI } & 931 \\ \text { MseI } & 948 \\ \text { MseI } & 1053 \\ \text { MseI } & 1094 \\ \text { MwoI } & 267 \\ \text { MwcI } & 279 \\ \text { MwoI } & 466 \\ \text { NlaIII } & 35 \\ \text { NlaIII } & 48 \\ \text { NlaIII } & 389 \\ \text { NlaIII } & 461 \\ \text { NlaIII } & 733 \\ \text { NlaIII } & 803 \\ \text { NlaIII } & 846 \\ \text { NlaIII } & 894 \\ \text { NlaIII } & 922 \\ \text { NlaIII } & 144 \\ \text { NlaIV } & 166 \\ \text { NspI } & 917 \\ \text { NspBII } & 946 \\ \text { NspBII } & \end{array}$




$\begin{array}{lr}\text { TaqIIa } & 180 \\ \text { TfiI } & 357 \\ \text { TfiI } & 503 \\ \text { TfiI } & 666 \\ \text { TseI } & 264 \\ \text { TseI } & 276 \\ \text { TseI } & 512 \\ \text { TseI } & 562 \\ \text { Tsp45I } & 178 \\ \text { Tsp45I } & 836 \\ \text { TspEI } & 540 \\ \text { TspEI } & 812 \\ \text { TspEI } & 850 \\ \text { TspEI } & 951 \\ \text { TspEI } & 1103 \\ \text { XhoII } & 477 \\ \text { XhoII } & 737 \\ \text { XmnI } & 652\end{array}$

The human interferon-alpha 1 gene restriction endonuclease recognition sites are summarized by enzyme.

\begin{tabular}{|c|c|c|}
\hline Name & Pattern & Sites \\
\hline AatII & gacgtc & 0 \\
\hline$A / C I$ & gtmkac & 0 \\
\hline P.CiI & $\operatorname{ccgc}$ & 0 \\
\hline AcII & aacgtt & 0 \\
\hline AcYI & grcgyc & 0 \\
\hline AfIII & cttaag & 0 \\
\hline AfIIII & acrygt & 1 \\
\hline AgeI & accggt & 0 \\
\hline AhaIII & tttaaa & 2 \\
\hline EIUI & agct & 4 \\
\hline AlwNI & cagnnnctg & $i$ \\
\hline ApaI & gggccc & 0 \\
\hline ApaBI & gcannnnntgc & 0 \\
\hline ApaII & gtgcac & 1 \\
\hline ApoI & raatty & 3 \\
\hline AscI & ggcgcgcc & 0 \\
\hline AsuI & ggnce & 5 \\
\hline AsuII & ttcgaa & 0 \\
\hline AvaI & cycgrg & 0 \\
\hline AvaII & ggwec & 4 \\
\hline AvaIII & atgoat & 0 \\
\hline AvrII & cctagg & 0 \\
\hline BaeI & acnnringtayc & 0 \\
\hline $\mathrm{BaII}$ & tggcca & 0 \\
\hline BanH I & ggatcc & 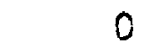 \\
\hline BhvI & gcagc & \\
\hline
\end{tabular}




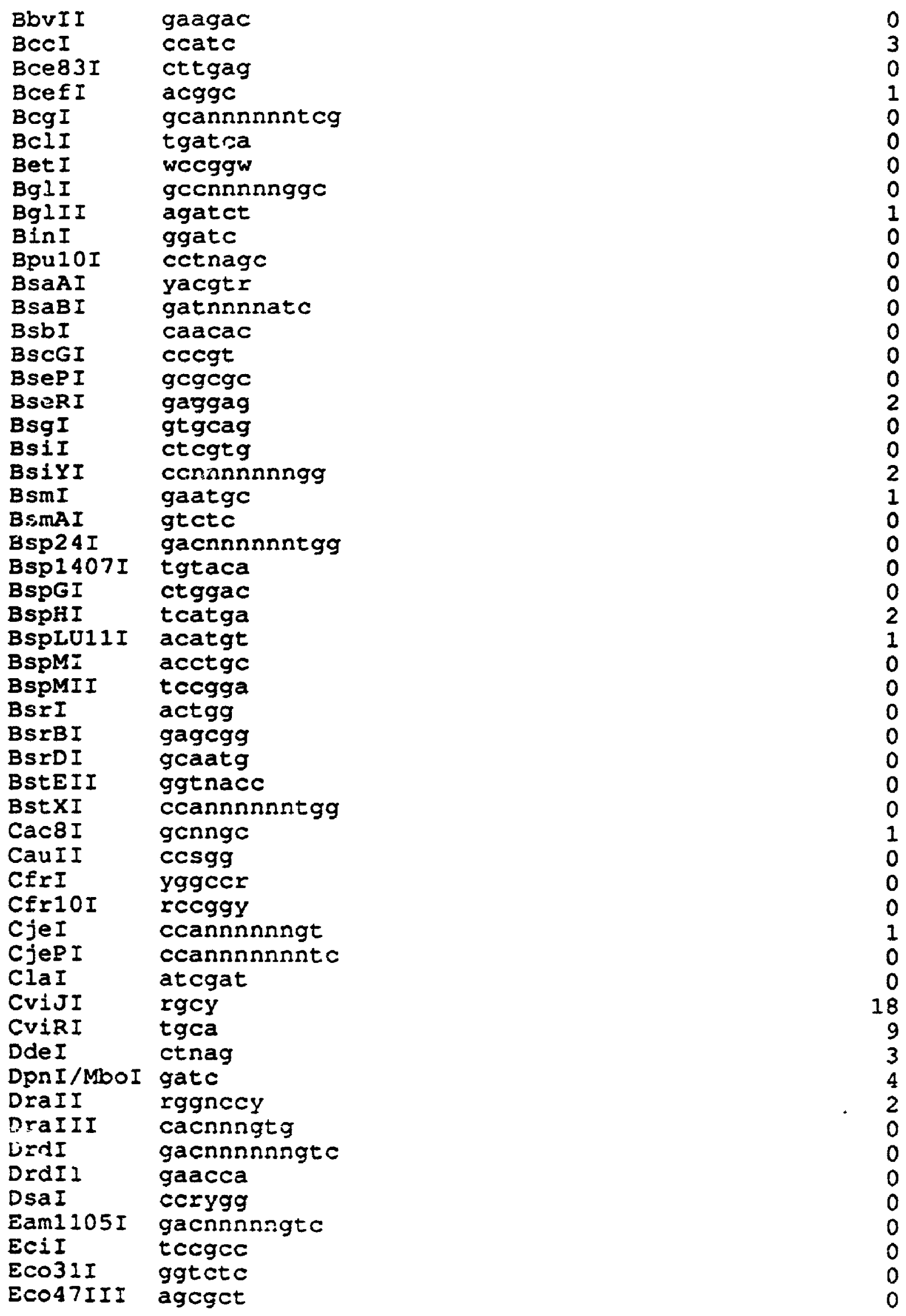




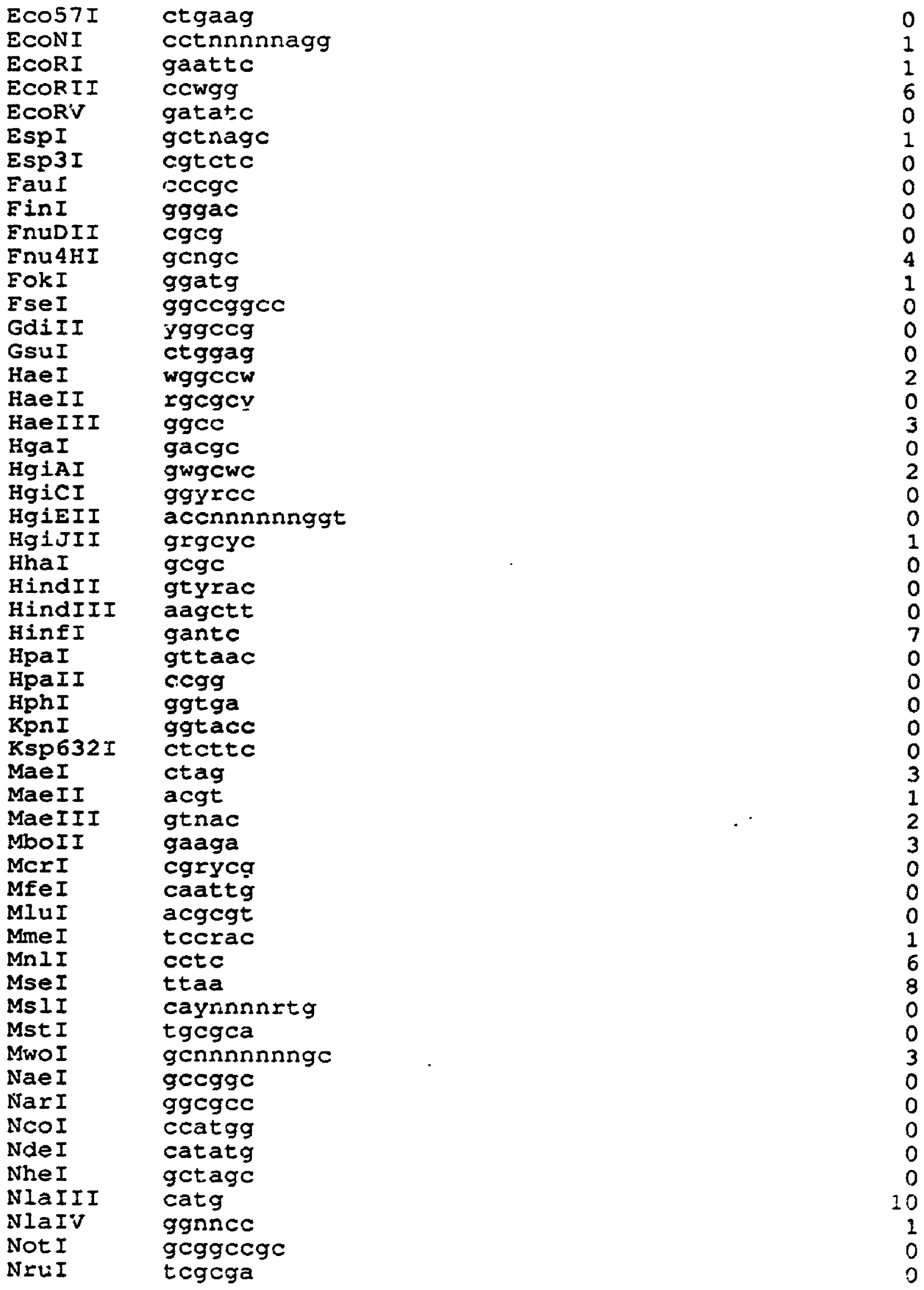




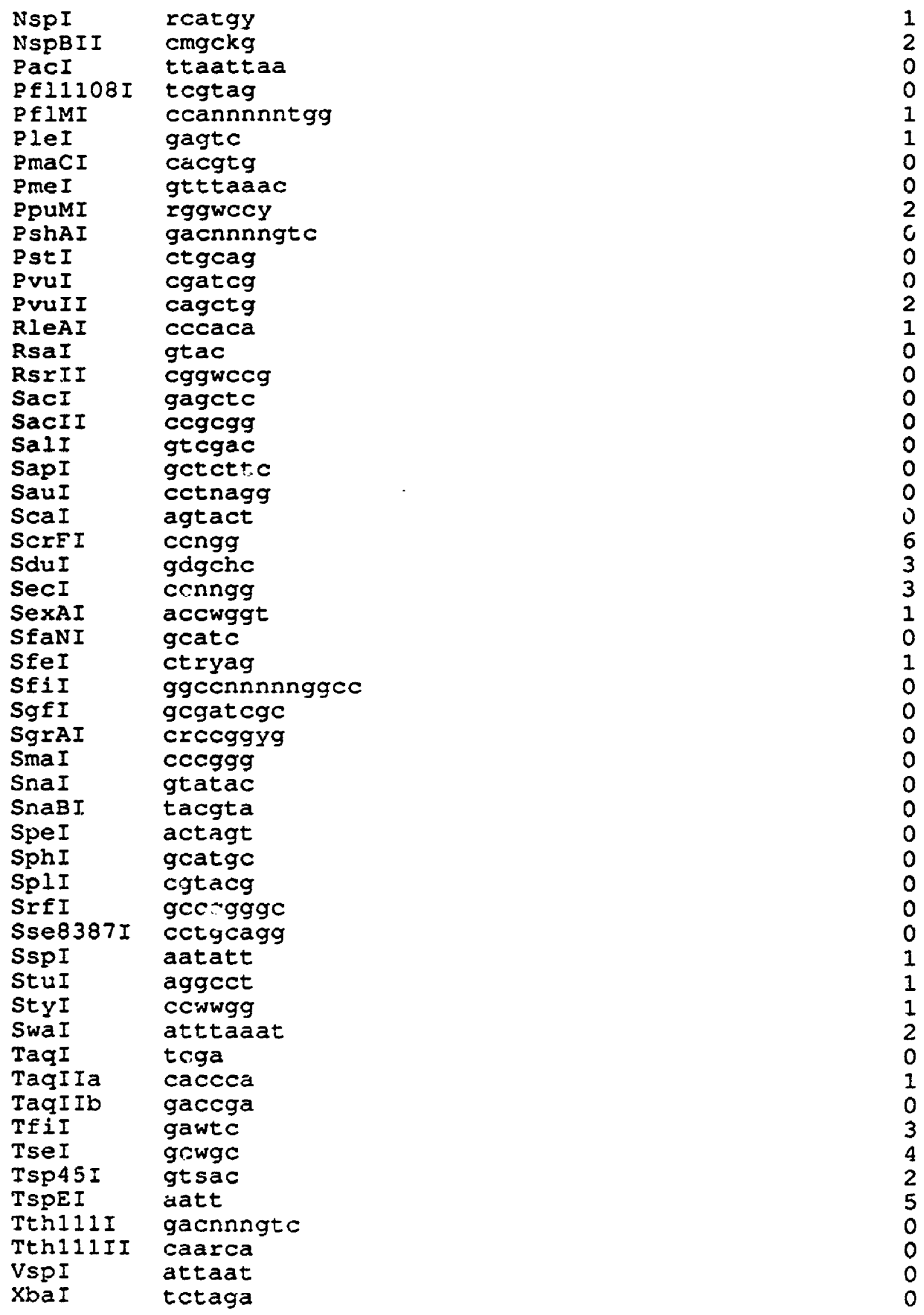


$\mathrm{XcmI}$

ccannnnnrinnntgg

0

XhoI

ctcgag

XhoII

XmaIII

rgatcy

cggccg

gaannnnttc

0

$X m n I$

Total 
Appendix E

\title{
Human Interferon Alpha-2 Gene
}

\author{
E.1 Nucleotide Sequence
}

The genomic DNA sequence of the human interferon-alpha 2 gene was retrieved from the Genbank sequence file humifnaa (Goeddel et. al., 1980) (Maeda et. al., 1980)

(Streuli et. al., 1980) (Goeddel et. al., 1981) (Lawn et. al., 1981b) (Bowden et. al., 1984)

(Benson et. al., 1993).

1 gcgcctctta tgtacccaca aaaatctatt ttcaaaaaag ttgctctaag aatatagtta

61 tcaagttaag taaestgtca atagccttt aattraatt ttaattgttt tarcattctt

121 tgcaataata aaacattaac tttatacttt ttaatttaat gtatagaata gagatataca

181 taggatatgt aaatagatac acagtgtata tgtgattaaa atataatggg agattcaatc

241 agaaaaaagt ttctaaaaag gctctggggt aaaagaggaa ggaaacaata atgaaaaaaa

301 tgtggtgaga aaaacagetg aaaacccatg taaagagtgt ataaagaaag caaaaagaga

36. agtagaaagt aacacagggg catttggaaa atgtaaacga gtatgttcce tatttaagge

421 taggcacaaa gcaaggtctt cagagaacct ggagcctaag gtttaggctc acccatttca

481 accagtctag cagcatctge aacatctaca atggecttga ccttgettt actggtggce

541 ctcetggtge tcagetgeaa gtcaagetge tctgtggget gtgatcty

601 agcctgggta gcaggaggac cttgatgctc ctggcacaga tgaggagaat ctctctttc

661 tcctgettga aggacagaca tgactttgga ttccccagg aggagtttgg caaccagttc

721 caaaaggetg aaaccatcce tgtectecat gagatgatce agcagatctt caatctcttc

781 agcacaaagg actcatctge tgcttgggat gagaccctce tagacaaatt ctacactgaa

841 ctctaccage agctgaatga cctggaagec tgtgtgatac agggggtggg ggtgacagag

901 actcccctga tgaaggagga ctccattctg getgtgagga aatacttcca aagaatcact

961 ctctatctga aagagaagaa atacagccet tgtgcctggg aggttgtcag agcagaaatc

1021 atgagatctt tttcttgtc aacaaacttg caagaaagtt taagaagtaa ggaatgaaaa

1081 ctggttcanc atggaaatga tttcattga tt-gtatgce agctcacett tttatgatct

1141 gecatttcaa agactcatgt ttctgctatg accatgacac gattaaatc trtcaaatg

1201 ttttnggag tattaatcain cattgtattc agctcttaag geactagtce cttacagagg

1261 accatgctga ctgatccalt atctatttaa atattttaa aarattatt atttaactat

1321 ttataanaca acttatttt gltcatatta tgtcatgtge acctttgcac agtggttaat

1381 gtaataaaat gtgttctttg tattggtaa atttatttg tgttgttcat tgaactttg

1441 ctatggaact tttgtacttg tttattcttt aanatgaaat tccaagccta attgtgeaac

1501 ctgattacag aataactggt acacttcatt tgtccatcan tattatattc aagatataag

1561 taanataaa cttctgtai accaagttgt atgttgtact caagataaca gggtgaacct

1621 aacaantaca attctgctct cttgtgtart tgattttgt atgaaaaaia ctaaaaatgg

1681 tantcatact taattatcag ttatggtaaa tggtatgaag agaagaagga acg 


\section{E.2 Restriction Endonuclease Sites}

The human interferon-alpha 2 gene restriction endonuclease recognition sites are arranged by ascending sequence location.

\begin{tabular}{|c|c|}
\hline \multicolumn{2}{|r|}{ Site } \\
\hline HhaI & 1 \\
\hline $\operatorname{Mn} 1 I$ & 4 \\
\hline RsaI & 12 \\
\hline RleAI & 15 \\
\hline DdeI & 46 \\
\hline MseI & 66 \\
\hline CViJI & 83 \\
\hline MseI & 89 \\
\hline TspEI & 91 \\
\hline MseI & 94 \\
\hline TspEI & 96 \\
\hline MseI & 101 \\
\hline TspeI & 103 \\
\hline CVIRI & 121 \\
\hline MseI & 136 \\
\hline MseI & 151 \\
\hline TspEI & 153 \\
\hline MseI & 156 \\
\hline MseI & 216 \\
\hline TfiI & 232 \\
\hline HinfI & 232 \\
\hline CviJI & 260 \\
\hline HphI & 304 \\
\hline PvuII & 315 \\
\hline NspBII & 315 \\
\hline AluI & 316 \\
\hline CviJI & 316 \\
\hline NlaIII & 327 \\
\hline MaeIII & 369 \\
\hline EcoNI & 409 \\
\hline BSIYI & 409 \\
\hline Mse I & 414 \\
\hline CVIJI & 41 \\
\hline MaeI & \\
\hline ECORII & \\
\hline SCIEI & \\
\hline GsuI & \\
\hline NlaIV & \\
\hline CViJI & \\
\hline SauI & \\
\hline Dde I & \\
\hline CVIJI & \\
\hline
\end{tabular}




\begin{tabular}{|c|c|}
\hline $\begin{array}{l}\text { TaqIIa } \\
\text { MaeI } \\
\text { BbvI } \\
\text { ApaBI } \\
\text { TseI } \\
\text { Fnu4HI } \\
\text { MwoI } \\
\text { AlwNI } \\
\text { SfaNI } \\
\text { CviRI } \\
\text { HaeI } \\
\text { HaeIII } \\
\text { CviJI } \\
\text { BsII } \\
\text { HaeIII } \\
\text { AsuI } \\
\text { CviJI } \\
\text { MnII } \\
\text { EcoRII } \\
\text { ScrEI } \\
\text { HgiAI } \\
\text { SduI } \\
\text { EspI } \\
\text { DdeI } \\
\text { PvuII } \\
\text { NspBII } \\
\text { AluI } \\
\text { CviJI } \\
\text { TseI } \\
\text { Fnu4HI } \\
\text { CvIRI } \\
\text { MwoI } \\
\text { AluI } \\
\text { CviJI } \\
\text { TseI } \\
\text { Fnu4HI } \\
\text { MwoI } \\
\text { CviJI } \\
\text { DpnI/MboI } \\
\text { Mn_I } \\
\text { RIeAI } \\
\text { PfIMI } \\
\text { BsiYI } \\
\text { CviJI } \\
\text { MwoI } \\
\text { EcoRII } \\
\text { ScrEI } \\
\text { SecI } \\
\text { PpuMI } \\
\text { DraII } \\
\text { AvaII } \\
\text { AsuI } \\
\text { EcoRII } \\
\text { ScrFI }\end{array}$ & $\begin{array}{l}470 \\
487 \\
490 \\
490 \\
490 \\
490 \\
490 \\
491 \\
493 \\
498 \\
512 \\
513 \\
513 \\
531 \\
537 \\
537 \\
537 \\
540 \\
543 \\
543 \\
547 \\
547 \\
549 \\
550 \\
552 \\
552 \\
553 \\
553 \\
554 \\
554 \\
556 \\
557 \\
565 \\
565 \\
566 \\
566 \\
569 \\
577 \\
603 \\
603 \\
616 \\
616 \\
617 \\
589 \\
593 \\
599 \\
607\end{array}$ \\
\hline
\end{tabular}




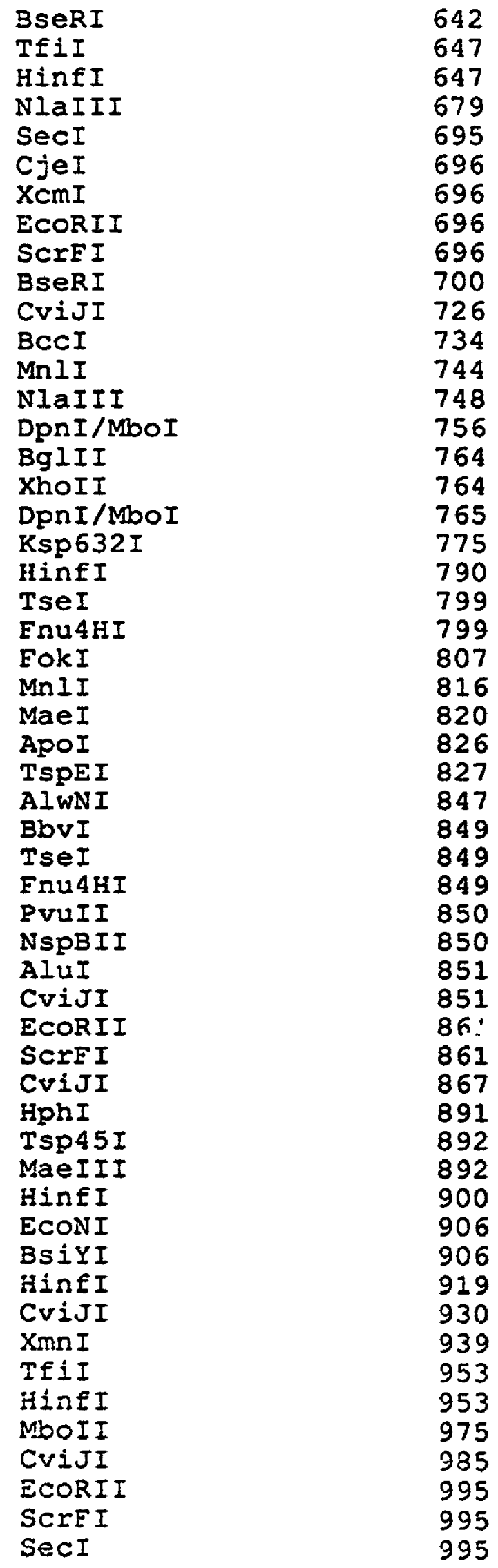




\begin{tabular}{|c|c|}
\hline 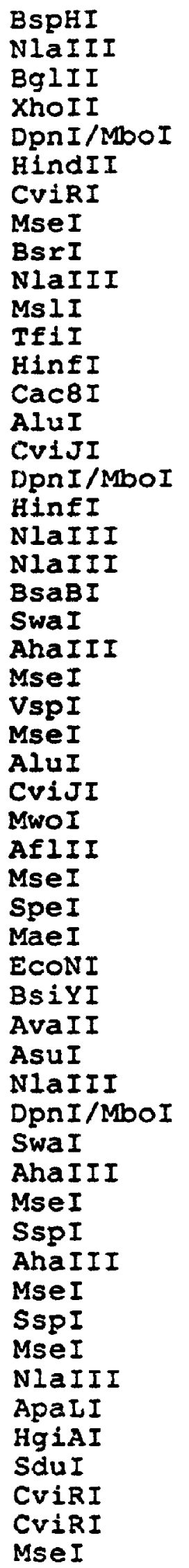 & $\begin{array}{l}1019 \\
1020 \\
102 \\
102 \\
1025 \\
1038 \\
1049 \\
1060 \\
1080 \\
1090 \\
1090 \\
110 \\
110 \\
1118 \\
112 \\
112 \\
113 \\
115 \\
115 \\
117 \\
118 \\
1182 \\
118 \\
118 \\
121 \\
121 \\
123 \\
123 \\
123 \\
123 \\
123 \\
124 \\
124 \\
1250 \\
1250 \\
125 \\
125 \\
126 \\
127 \\
128 \\
128 \\
128 \\
129 \\
129 \\
129\end{array}$ \\
\hline
\end{tabular}




$\begin{array}{ll}\text { ApoI } & 1409 \\ \text { TspEI } & 1410 \\ \text { RsaI } & 1454 \\ \text { AhaIII } & 1468 \\ \text { MseI } & 1469 \\ \text { ApoI } & 1477 \\ \text { TspEI } & 1478 \\ \text { CviJI } & 1485 \\ \text { TspEI } & 1490 \\ \text { CviRI } & 1495 \\ \text { BsrI } & 1515 \\ \text { RsaI } & 1519 \\ \text { BCCI } & 1534 \\ \text { SspI } & 1539 \\ \text { RsaI } & 1596 \\ \text { HphI } & 1612 \\ \text { TspEI } & 1630 \\ \text { MseI } & 1690 \\ \text { TspEI } & 1692 \\ \text { MboII } & 1717 \\ \text { MboII } & 1722\end{array}$

The human interferon-alpha 2 gene restriction endonuclease recognition sites are arranged by enzyme.

$\begin{array}{lr}\text { Name } & \text { Site } \\ \text { AfIII } & 1235 \\ \text { AhaIII } & 1183 \\ \text { AhaIII } & 1286 \\ \text { AhaIII } & 1296 \\ \text { AhaIII } & 1468 \\ \text { AluI } & 316 \\ \text { AluI } & 553 \\ \text { AluI } & 565 \\ \text { AluI } & 851 \\ \text { AluI } & 1121 \\ \text { AluI } & 1231 \\ \text { AlwNI } & 491 \\ \text { AlwNI } & 847 \\ \text { ApaBI } & 490 \\ \text { ApaII } & 1357 \\ \text { ApoI } & 826 \\ \text { ApoI } & 1409 \\ \text { ApoI } & 1477 \\ \text { AsuI } & 537 \\ \text { AsuI } & 617 \\ \text { AsuI } & 1259 \\ \text { AvaII } & 617 \\ \text { AvaII } & 1259\end{array}$




$\begin{array}{lr}\text { BbvI } & 490 \\ \text { BbvI } & 849 \\ \text { BCCI } & 734 \\ \text { BCCI } & 1534 \\ \text { BgIII } & 764 \\ \text { BgIII } & 7024 \\ \text { BsaBI } & 1024 \\ \text { BserI } & 1181 \\ \text { BseRI } & 642 \\ \text { BsiYI } & 700 \\ \text { BsiYI } & 409 \\ \text { BsiYI } & 597 \\ \text { BsiYI } & 906 \\ \text { BapHI } & 1250 \\ \text { BsII } & 1019 \\ \text { BsrI } & 531 \\ \text { BsrI } & 1080 \\ \text { Cac8I } & 1515 \\ \text { CjEI } & 1118 \\ \text { CviJI } & 696 \\ \text { CviJI } & 83 \\ \text { CviJI } & 260 \\ \text { CviJI } & 316 \\ \text { CviJI } & 418 \\ \text { CVIJI } & 465 \\ \text { CviJI } & 1025 \\ \text { CviJI } & 136 \\ \text { CviJI } & \end{array}$




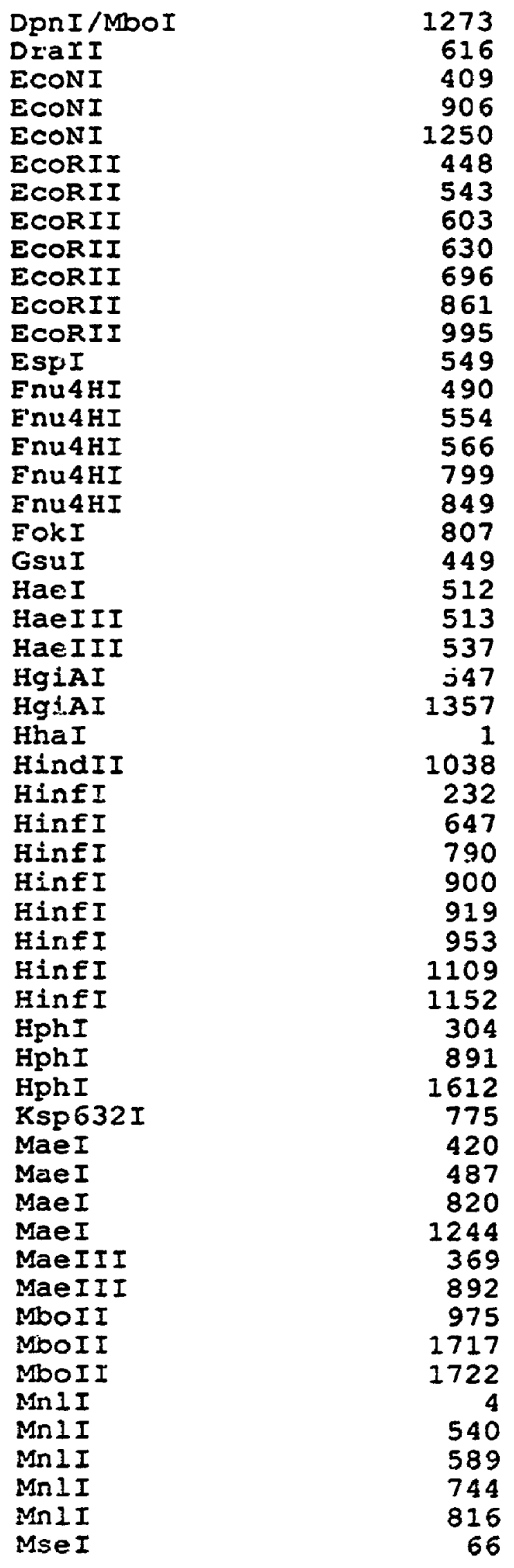




$\begin{array}{lr}\text { MseI } & 89 \\ \text { MseI } & 94 \\ \text { MseI } & 101 \\ \text { MseI } & 136 \\ \text { MseI } & 151 \\ \text { MseI } & 156 \\ \text { MseI } & 216 \\ \text { MseI } & 414 \\ \text { MseI } & 1060 \\ \text { MseI } & 1184 \\ \text { MseI } & 1213 \\ \text { MseI } & 1236 \\ \text { MseI } & 1287 \\ \text { MseI } & 1297 \\ \text { MseI } & 1313 \\ \text { MseI } & 1376 \\ \text { MseI } & 1469 \\ \text { MseI } & 1690 \\ \text { MsII } & 1090 \\ \text { MwoI } & 490 \\ \text { MwoI } & 557 \\ \text { MwoI } & 515 \\ \text { MwoI } & 569 \\ \text { MwoI } & 648 \\ \text { NlaIII } & 543 \\ \text { NlaIII } & 603 \\ \text { NlaIII } & 630 \\ \text { NlaIII } & 696 \\ \text { NlaIII } & \end{array}$




\begin{tabular}{|c|c|}
\hline $\begin{array}{l}\text { ScrEI } \\
\text { ScrEI } \\
\text { SduI } \\
\text { SduI } \\
\text { SecI } \\
\text { SecI } \\
\text { SecI } \\
\text { SfaNI } \\
\text { SpeI } \\
\text { SspI } \\
\text { SspI } \\
\text { SspI } \\
\text { SwaI } \\
\text { SwaI } \\
\text { TaqII } \\
\text { TfiI } \\
\text { TfiI } \\
\text { TfiI } \\
\text { TfiI } \\
\text { TseI } \\
\text { TseI } \\
\text { TseI } \\
\text { TseI } \\
\text { TseI } \\
\text { Tsp45I } \\
\text { TspeI } \\
\text { TspEI } \\
\text { TspEI } \\
\text { TspEI } \\
\text { TspEI } \\
\text { TspeI } \\
\text { TspEI } \\
\text { TspEI } \\
\text { TspEI } \\
\text { TspEI } \\
\text { VspI } \\
\text { XcmI } \\
\text { XhoII } \\
\text { XhoII } \\
\text { XmnI }\end{array}$ & $\begin{array}{r}861 \\
995 \\
547 \\
1357 \\
603 \\
695 \\
995 \\
493 \\
1243 \\
1290 \\
1301 \\
1539 \\
1182 \\
1385 \\
470 \\
232 \\
647 \\
953 \\
1109 \\
490 \\
554 \\
566 \\
799 \\
849 \\
892 \\
91 \\
06 \\
103 \\
153 \\
827 \\
1410 \\
1478 \\
1490 \\
1630 \\
1692 \\
1212 \\
606\end{array}$ \\
\hline
\end{tabular}

The human interferon-alpha 2 gene restriction endonuclease recognition sites are summarized by enzyme.

$\begin{array}{llr}\text { Name } & \text { Pattern } & \text { Sites } \\ \text { AatII } & \text { gacgtc } & 0 \\ \text { AccI } & \text { gtmkac } & 0 \\ \text { AciI } & \text { ccgc } & 0 \\ \text { AclI } & \text { aacgtt } & 0\end{array}$




\begin{tabular}{|c|c|}
\hline 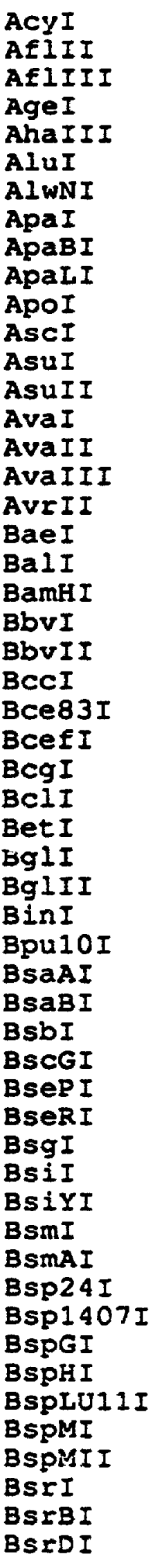 & $\begin{array}{l}\text { grcgyc } \\
\text { cttaag } \\
\text { acrygt } \\
\text { accggt } \\
\text { ttaaa } \\
\text { agct } \\
\text { cagnnnctg } \\
\text { gggccc } \\
\text { gcannnntgc } \\
\text { gtgcac } \\
\text { raatty } \\
\text { ggcgcgcc } \\
\text { ggnce } \\
\text { ttcgaa } \\
\text { cycgrg } \\
\text { ggwcc } \\
\text { atgcat } \\
\text { cctagg } \\
\text { acnnnngtayc } \\
\text { tggcca } \\
\text { ggatcc } \\
\text { gcagc } \\
\text { gaagac } \\
\text { ccatc } \\
\text { cttgag } \\
\text { acggc } \\
\text { gcannnnnntcg } \\
\text { tgatca } \\
\text { wccggw } \\
\text { gccnnnnnggc } \\
\text { agatct } \\
\text { ggatc } \\
\text { cctnagc } \\
\text { yacgtr } \\
\text { gatnnnnatc } \\
\text { caacac } \\
\text { cccgt } \\
\text { gcgcgc } \\
\text { gaggag } \\
\text { gtgcag } \\
\text { ctcgtg } \\
\text { ccnnnnnngg } \\
\text { gaatgc } \\
\text { gtctc } \\
\text { gacnnnnntgg } \\
\text { tgtaca } \\
\text { ctggac } \\
\text { tcatga } \\
\text { acatgt } \\
\text { acctgc } \\
\text { tccgga } \\
\text { actgg } \\
\text { gagcgg } \\
\text { gcaatg } \\
\text { gaationg }\end{array}$ \\
\hline
\end{tabular}




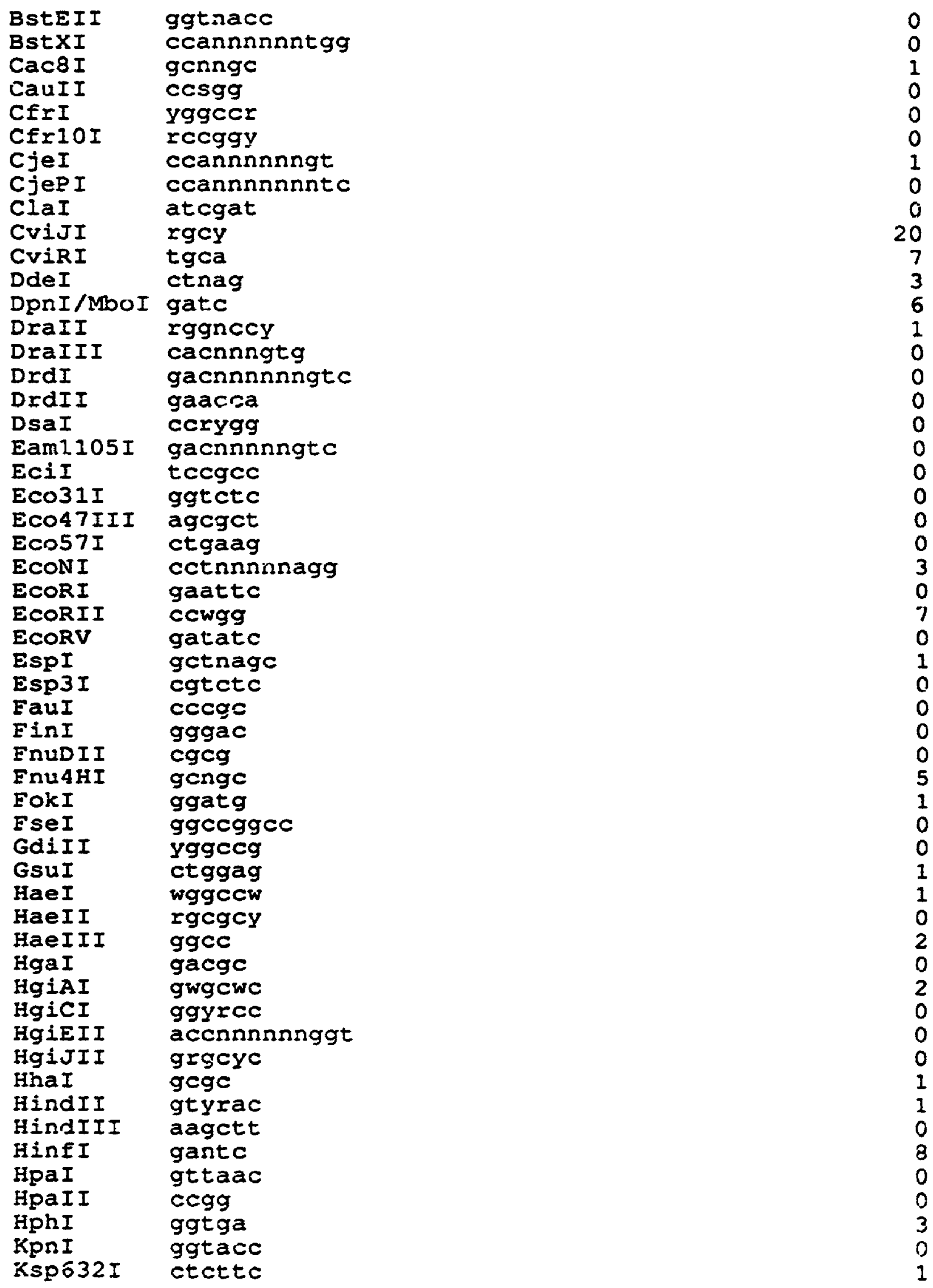




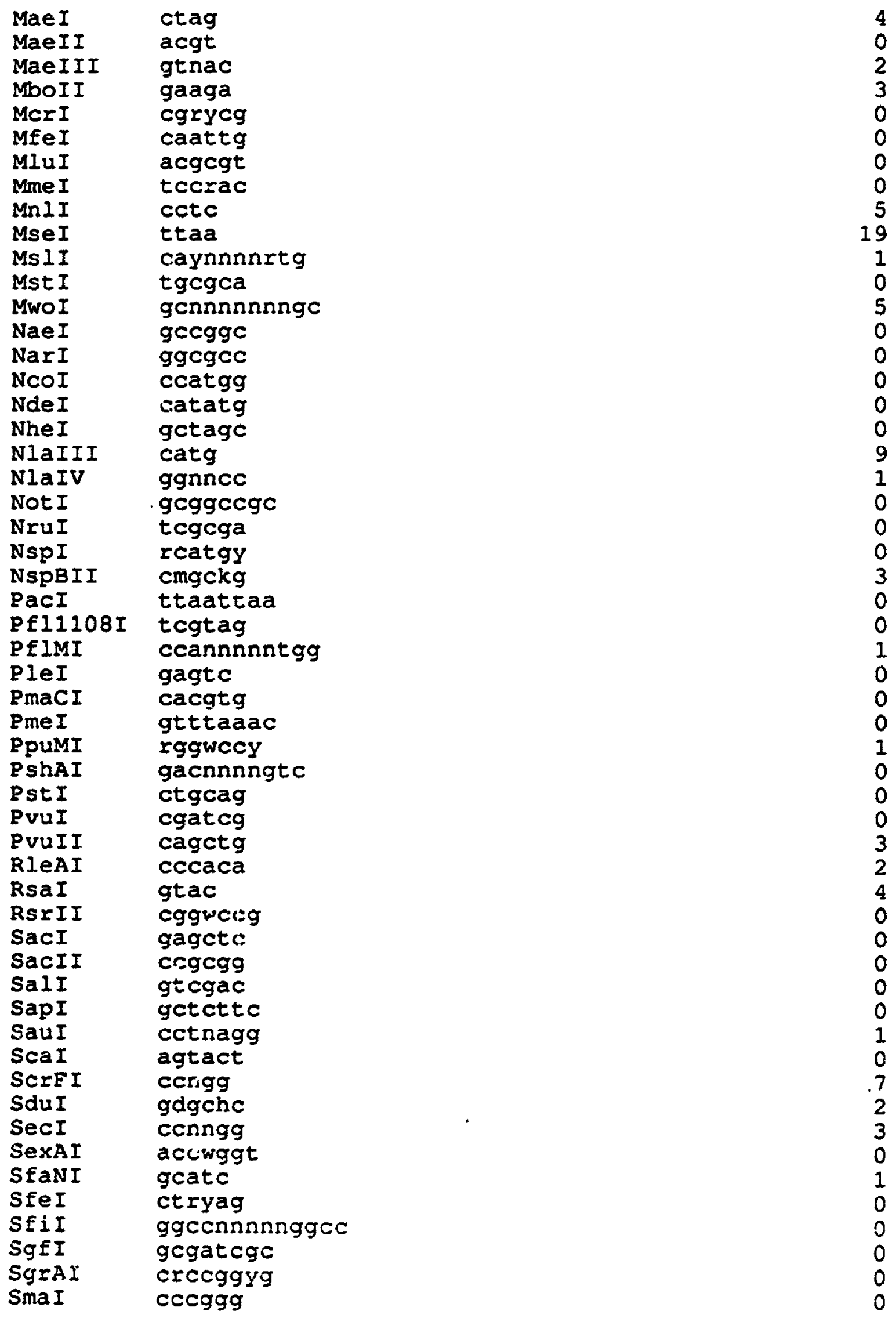


\begin{tabular}{llr} 
SnaI & gtatac & 0 \\
SnaBI & tacgta & 0 \\
SpeI & actagt & 1 \\
SphI & gcatgc & 0 \\
SpII & cgtacg & 0 \\
SrfI & gcccgggc & 0 \\
Sse8387I & cctgcagg & 0 \\
SspI & aatatt & 3 \\
StuI & aggcct & 0 \\
StyI & ccwwgg & 0 \\
SwaI & atttaaat & 2 \\
TaqI & tcga & 0 \\
TaqIIa & caccca & 1 \\
TaqIIb & gaccga & 0 \\
TfiI & gawtc & 4 \\
TseI & gcwgc & 5 \\
Tsp45I & gtsac & 1 \\
TspEI & aatt & 1 \\
TthIIII & gacnnngtc & 0 \\
ThIIIII & caarca & 0 \\
VspI & attaat & 0 \\
XbaI & tctaga & 1 \\
XcmI & ccanrinnnnnntgg & 0 \\
XhoI & ctcgag & 1 \\
XhoII & rgatcy & 0 \\
XmaIII & cggccg & 2 \\
XmI & gaannnnttc & 0 \\
Total & & 1 \\
& & \\
\hline & & 25
\end{tabular} 


\title{
Appendix F
}

\section{Human Interferon-Alpha 4 Gene}

\author{
F.1 Nucleotide Sequence
}

The genomic DNA sequence of the human interferon-alpha 4 gene was retrieved from the Geribank sequence file hsifna4b (Henco et. al., 1985) (Benson et. al., 1993).

1 aatgcaaata gcttatatta tatgatttat ttctagtaaa gttattcaac acatcagtac

61 ttatgtcaag tgctgaaaag aanaaagtgt tggcaacate tggatgaata ctgcagctga

121 tgaagtttac aaattattt gtcatataaa gcaaaattca aagcttcata cactaagaga

181 aaaattttaa aaaattattc attcatattt ttaggagttt tgaatgattg gatatgtaat

241 tatattcata ttattaatgt gtatctatat agatttttat tttgcatatg tactttgata

301 caaanttac atgaacaaat tacactaaaa gttattccac aaatatactt atcaaattaa

361 gttaaatgtc aatagcttt aaacttaiat tttagtttaa cttttctgtc attcttact

421 ttgaataaaa agagcaaact ttgtagtttt tatctgtgaa gtagaggtat acgtaatata

481 cataaataga tatgccaant ctgtgttatt aaaattcat gaagatttca attagaaaaa

541 aataccataa aaggcttga gtgcaggtga aaaataggca atgatgaaaa aaatgaaa

601 acttttaaa cacatgtaga gagtgcgtaa agaaagcaaa aacagagata gaaagtacaa

661 ctagggaatt tagaaaatgg aaattagtat gttcactatt taagacctat gcacagagca

721 aagtcttcag aaaacctaga ggccgaagtt caaggttatc catctcaagt agcctagcaa

781 tattgcaac atcccaatgg cectgtcctt ttctttactg atggecgtge tggtgctcag

841 ctacaaa:ce atctgttctc tgggetgtga tctgcctcag acccacagec tgggtaatag

901 gagggecttg atactcetgg cacaaatggg aagaatctct cattctcct gectgaagga

961 cagacatgat ttcggattcc ccgaggagga gtttgatggc caccagttcc agaagactca

1021 agccatctct gtcctccatg agatgatcca geagaccttc aatctcttca gcacagagga

1081 ctcatctgct gettgggaac agagcctect agaaaaatt tccactgaac tttaccagca

1141 actgaatgac ctggaagcat gtgtgataca ggaggttggg gtggaagaga ctccctgar

1201 gaatgtggac tccatcctgg ctgtgaggaa atacttccaa agaatcactc ttatctaac

1261 agagaagaaa tacagccctt gtgectggga ggttgtcaga gcagaaatca tgagatccet

1321 ctcgtttca acaaacttgc aaaaagatt aaggaggaag gattgaaacc tggttcaaca

1381 tggaaatgat cctgactgac taatacatta tctcacac.. tcatgagtte ttccatttca

1441 angactcact tctatancca ccacgagttg aatcaaaatt ttcaaatgtt ttcagcagtg

1501 tgaagaagct tggtgtatac ctgtgcaggc actagtcctt tacagatgac aatgctgatg

1561 tctctgttca tctattratt taaatattta tttattttta aaatttahat tatttttat

1621 grgatatcat gagtaccttt acattgtggt gaatgtaaca atatatgttc ttcatattta

1681 gecaatatat tanttcctt ttcattaaa ttttactat acaaauttt tgtgttgtt

1741 tattccttaa gatas datge canggetgac ttacaacet gacttailaaa tagatgattt

1801 aattaagtta cctatcataa tttattcan gttataaaaa aacatatttt tctgtarctg

1861 gttatatgtt gccttcagga tatnaatgtg aacataaaat atacagtcce tcttctcttg

1921 tatctttat tttgtcagg aaagaaatct aaaaacaata atantgctga attaatatca

1981 gtgatgctaa ctgctataat gtgaggaagt aaaaaaacaa tg 


\section{F.2 Restriction Endonuclease Sites}

The human interferon-alpha 4 gene restriction endonuclease recognition sites are arranged by ascending sequence location.

$\begin{array}{lr}\text { Name } & \text { site } \\ \text { CviRI } & 3 \\ \text { AluI } & 10 \\ \text { CviJI } & 10 \\ \text { MaeI } & 33 \\ \text { BsbI } & 47 \\ \text { ScaI } & 56 \\ \text { RsaI } & 57 \\ \text { MsII } & 97 \\ \text { FokI } & 102 \\ \text { PstI } & 111 \\ \text { SfeI } & 111 \\ \text { CviRI } & 112 \\ \text { BbvI } & 113 \\ \text { TseI } & 113 \\ \text { Fnu4HI } & 113 \\ \text { PvuII } & 114 \\ \text { NspBII } & 114 \\ \text { AluI } & 115 \\ \text { CvIJI } & 115 \\ \text { TspEI } & 132 \\ \text { ApoI } & 154 \\ \text { TspEI } & 155 \\ \text { HindIII } & 161 \\ \text { AluI } & 162 \\ \text { CviJI } & 162 \\ \text { DdeI } & 173 \\ \text { ApoI } & 182 \\ \text { TspEI } & 183 \\ \text { AhaIII } & 186 \\ \text { MseI } & 187 \\ \text { TspEI } & 193 \\ \text { TspEI } & 238 \\ \text { VspI } & 253 \\ \text { MseI } & 254 \\ \text { CviRI } & 283 \\ \text { NdeI } & 285 \\ \text { RsaI } & 290 \\ \text { ApoI } & 303 \\ \text { TspEI } & 304 \\ \text { NlaIII } & 310 \\ \text { TspEI } \\ \text { TsptI } & 318 \\ & 355\end{array}$




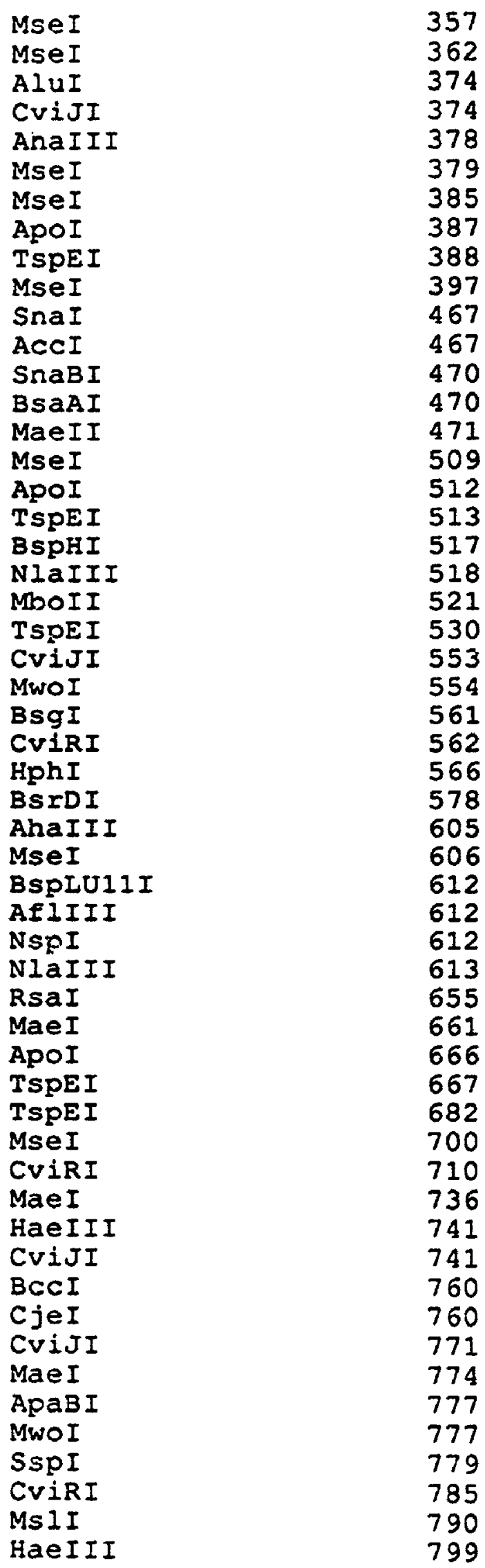




\begin{tabular}{|c|c|}
\hline 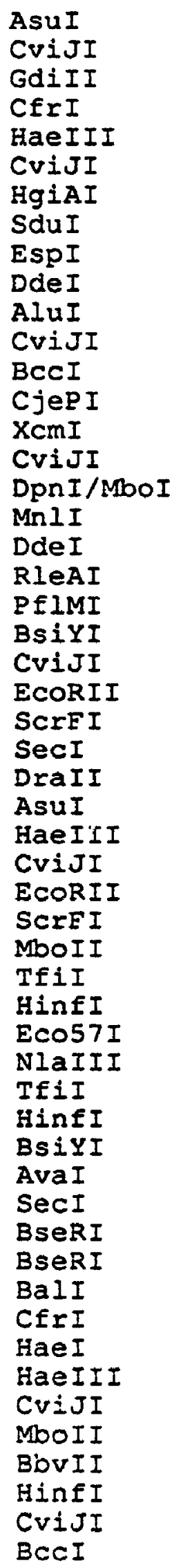 & $\begin{array}{l}799 \\
799 \\
822 \\
822 \\
823 \\
823 \\
833 \\
833 \\
835 \\
836 \\
839 \\
839 \\
849 \\
849 \\
849 \\
863 \\
869 \\
875 \\
876 \\
882 \\
883 \\
883 \\
887 \\
889 \\
889 \\
889 \\
902 \\
903 \\
904 \\
904 \\
916 \\
916 \\
930 \\
933 \\
933 \\
953 \\
965 \\
975 \\
975 \\
979 \\
980 \\
981 \\
983 \\
986 \\
997 \\
997 \\
997 \\
998 \\
998 \\
1012 \\
1012 \\
1015 \\
1021 \\
1023\end{array}$ \\
\hline
\end{tabular}




\begin{tabular}{|c|c|}
\hline 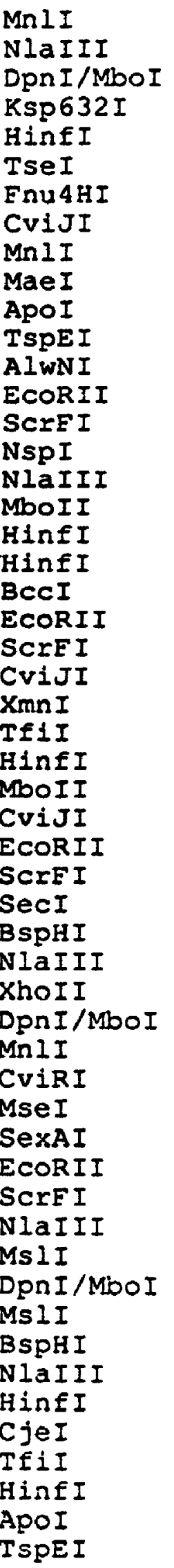 & $\begin{array}{l}1033 \\
1037 \\
1045 \\
1064 \\
1079 \\
1088 \\
1088 \\
1103 \\
1105 \\
1109 \\
1115 \\
1116 \\
1136 \\
1150 \\
1150 \\
1157 \\
1158 \\
1184 \\
1189 \\
1208 \\
1212 \\
1216 \\
1216 \\
1219 \\
1228 \\
1242 \\
1242 \\
1264 \\
1274 \\
1284 \\
1284 \\
1284 \\
1308 \\
1309 \\
1313 \\
1314 \\
1318 \\
1338 \\
1349 \\
1368 \\
1369 \\
1369 \\
1379 \\
1379 \\
1388 \\
1416 \\
1421 \\
1422 \\
1443 \\
1458 \\
1470 \\
1470 \\
1476 \\
1477\end{array}$ \\
\hline
\end{tabular}




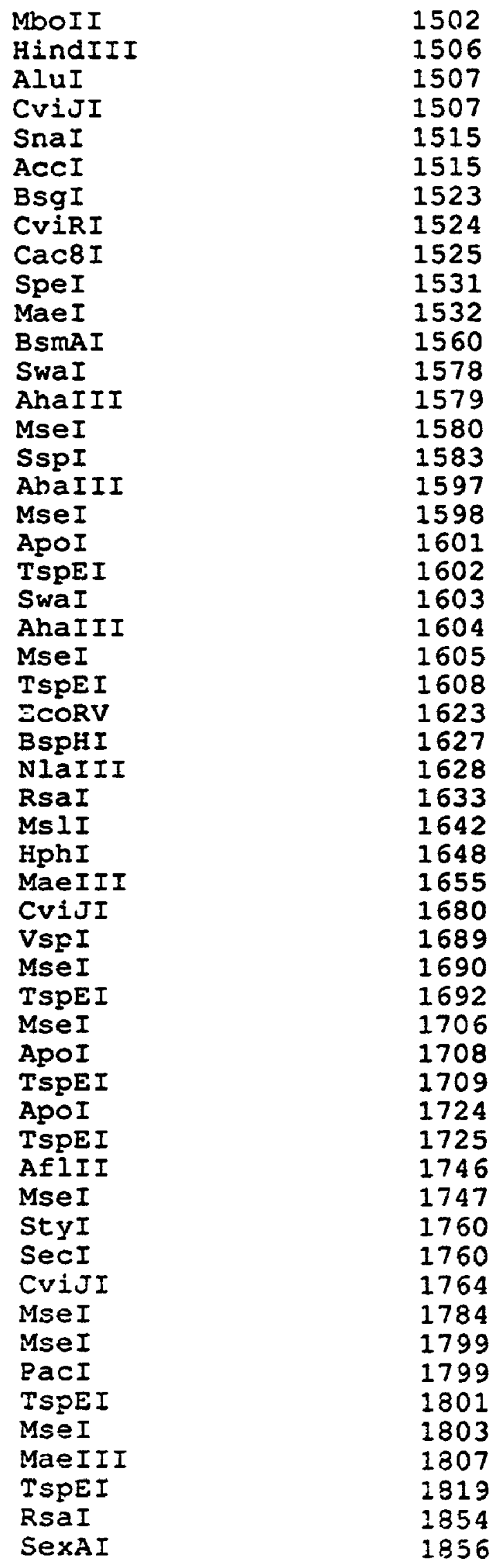




$\begin{array}{ll}\text { ECORII } & 1857 \\ \text { SCIEI } & 1857 \\ \text { MnII } & 1909 \\ \text { KSp632I } & 1910 \\ \text { TSPEI } & 1970 \\ \text { VSPI } & 1971 \\ \text { MseI } & 1972\end{array}$

The human interferon-alpha 4 gene restriction endonuclease recognition sites are arranged by enzyme.

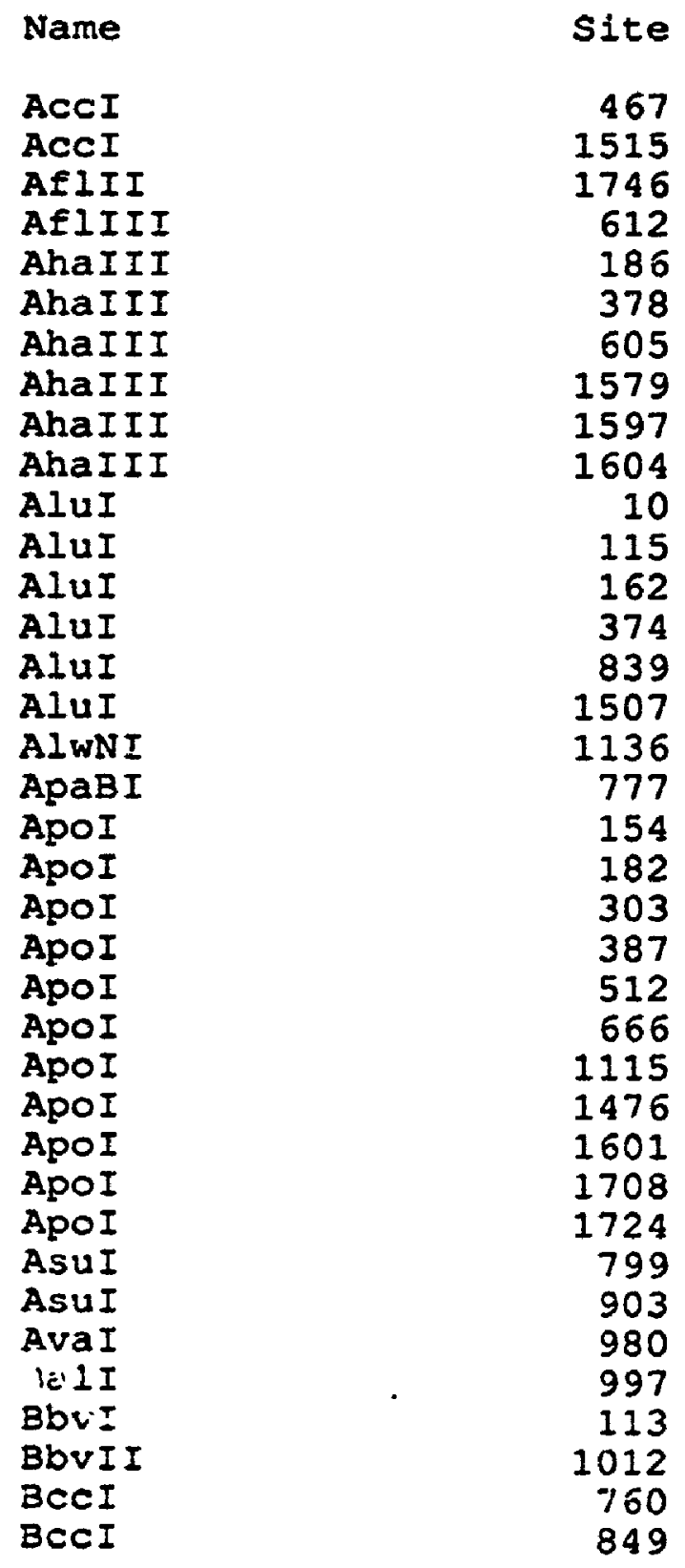




\begin{tabular}{|c|c|}
\hline $\begin{array}{l}\text { BCCI } \\
\text { BCCI } \\
\text { BsaAI } \\
\text { BsbI } \\
\text { BseRI } \\
\text { BseRI } \\
\text { BsgI } \\
\text { BsgI } \\
\text { BsiYI } \\
\text { BsiYI } \\
\text { BsmaI } \\
\text { BspHI } \\
\text { BspHI } \\
\text { BspHI } \\
\text { BspHI } \\
\text { BspIUIII } \\
\text { BsrDI } \\
\text { Cac8I } \\
\text { CfrI } \\
\text { CfrI } \\
\text { CjeI } \\
\text { CjeI } \\
\text { CjeRI } \\
\text { CviJI } \\
\text { CviJI } \\
\text { CviJI } \\
\text { CviJI } \\
\text { CviJI } \\
\text { CviJI } \\
\text { CviJI } \\
\text { CviJI } \\
\text { CviJI } \\
\text { CviJI } \\
\text { CviJI } \\
\text { CviJI } \\
\text { CviJI } \\
\text { CviJI } \\
\text { CviJI } \\
\text { CviJI } \\
\text { CviJI } \\
\text { CviJI } \\
\text { CviJI } \\
\text { CviJI } \\
\text { CviJI } \\
\text { CviRI } \\
\text { CviRI } \\
\text { CviRI } \\
\text { CviRI } \\
\text { CviRI } \\
\text { CviRI } \\
\text { CviRI } \\
\text { CviRI } \\
\text { DdeI } \\
\text { DdeI }\end{array}$ & $\begin{array}{r}1023 \\
1212 \\
470 \\
47 \\
983 \\
986 \\
561 \\
1523 \\
883 \\
979 \\
1560 \\
517 \\
1308 \\
1421 \\
1627 \\
612 \\
578 \\
1525 \\
822 \\
997 \\
760 \\
1458 \\
84\end{array}$ \\
\hline
\end{tabular}




$\begin{array}{lr}\text { DdeI } & 876 \\ \text { DpnI/MboI } & 869 \\ \text { DpnI/MboI } & 1045 \\ \text { DpnI/MboI } & 1314 \\ \text { DpnI/MboI } & 1388 \\ \text { DraII } & 902 \\ \text { Eco57I } & 953 \\ \text { EcoRII } & 889 \\ \text { EcoRII } & 916 \\ \text { EcoRII } & 1150 \\ \text { EcofII } & 1216 \\ \text { EcoRII } & 1284 \\ \text { EcoRII } & 1369 \\ \text { EcoRII } & 1857 \\ \text { EcoRV } & 1623 \\ \text { EspI } & 835 \\ \text { Env4HI } & 113 \\ \text { Enu4HI } & 1088 \\ \text { EokI } & 102 \\ \text { GdiII } & 822 \\ \text { HaeI } & 997 \\ \text { HaeIII } & 741 \\ \text { HaeIII } & 799 \\ \text { HaeIII } & 823 \\ \text { HaeIII } & 1012 \\ \text { HaeIII } & \end{array}$




\begin{tabular}{|c|c|}
\hline $\begin{array}{l}\text { MboII } \\
\text { MboII } \\
\text { MboII } \\
\text { MnII } \\
\text { MnII } \\
\text { MnII } \\
\text { MnII } \\
\text { MnII } \\
\text { MseI } \\
\text { MseI } \\
\text { MseI } \\
\text { MseI } \\
\text { MseI } \\
\text { MseI } \\
\text { MseI } \\
\text { MseI } \\
\text { MseI } \\
\text { MseI } \\
\text { MseI } \\
\text { MseI } \\
\text { MseI } \\
\text { MseI } \\
\text { MseI } \\
\text { MseI } \\
\text { MseI } \\
\text { MseI } \\
\text { MseI } \\
\text { MseI } \\
\text { MseI } \\
\text { MslI } \\
\text { MsII } \\
\text { MsII } \\
\text { MsII } \\
\text { MsII } \\
\text { MwoI } \\
\text { MwoI } \\
\text { NdeI } \\
\text { NIaIII } \\
\text { NlaIII } \\
\text { NlaIII } \\
\text { NlaIII } \\
\text { NlaIII } \\
\text { NlaIII } \\
\text { NlaIII } \\
\text { NlaIII } \\
\text { NlaIII } \\
\text { NlaIII } \\
\text { NspI } \\
\text { NspI } \\
\text { NspBII } \\
\text { PacI } \\
\text { PfIMI } \\
\text { PstI } \\
\text { PvuII }\end{array}$ & $\begin{array}{r}1184 \\
1264 \\
1502 \\
875 \\
1033 \\
1105 \\
1318 \\
1909 \\
187 \\
254 \\
357 \\
362 \\
379 \\
385 \\
397 \\
509 \\
606 \\
700 \\
1349 \\
1580 \\
1598 \\
1605 \\
1690 \\
1706 \\
1747 \\
1784 \\
1799 \\
1803 \\
1972 \\
179 \\
179 \\
790 \\
1379 \\
1416 \\
1642 \\
1111 \\
1114\end{array}$ \\
\hline
\end{tabular}




$\begin{array}{lr}\text { RleAI } & 882 \\ \text { RsaI } & 57 \\ \text { RsaI } & 290 \\ \text { RsaI } & 655 \\ \text { RsaI } & 1633 \\ \text { RsaI } & 1854 \\ \text { ScaI } & 56 \\ \text { ScrEI } & 889 \\ \text { ScrEI } & 916 \\ \text { ScrEI } & 1150 \\ \text { ScrEI } & 1216 \\ \text { ScrEI } & 1284 \\ \text { ScrEI } & 1369 \\ \text { ScrEI } & 1857 \\ \text { SduI } & 833 \\ \text { SecI } & 889 \\ \text { SecI } & 981 \\ \text { SecI } & 1284 \\ \text { SecI } & 1760 \\ \text { SexAI } & 1368 \\ \text { SexAI } & 1856 \\ \text { SfeI } & 111 \\ \text { SnaI } & 467 \\ \text { SnaI } & 1515 \\ \text { SnaBI } & 470 \\ \text { SpeI } & 1531 \\ \text { SspI } & 779 \\ \text { SspI } & 1116 \\ \text { StyI } & 1477 \\ \text { SwaI } & 1602 \\ \text { SwaI } & 1608 \\ \text { TfiI } & \end{array}$




$\begin{array}{lr}\text { TSPEI } & 1692 \\ \text { TSPEI } & 1709 \\ \text { TSPEI } & 1725 \\ \text { TSpEI } & 1801 \\ \text { TSpEI } & 1819 \\ \text { TspEI } & 1970 \\ \text { VspI } & 253 \\ \text { VspI } & 1689 \\ \text { VspI } & 1371 \\ \text { XcmI } & 849 \\ \text { XhoII } & 1313 \\ \text { XmnI } & 1228\end{array}$

The human interferon-alpha 4 gene restriction endonuclease recognition sites are summarized by enzyme.

Name

Pattern

gacgtc

Aat I

AccI

AciI

ACII

AcyI

AfIII

AfIIII

AgeI

AhaIII

AluI

AlwNI

Apa I

ApaBI

ApaLI

ApoI

AscI

AsuI

AsuII

Ava I

AvaII

AvaIII

AvrII

BaeI

BalI

BamiI

BbvI

BbvII

BCCI

Bce83I

BcefI

BCgI

BCII

gtgcac gtmkac

ccgc

aacgtt

grcgyc

cttaag

acrygt

accggt

tttaaa

agct

cagnnnctg

gggccc

gcannnnntgc

raatty

ggcgcgce

ggnce

ttcgaa

cycgrg

ggwce

atgcat

cctagg

acnnnngtayc

tggcca

ggatcc

gcagc

gaagac

ccatc

cttgag

acggc

gcannnsantcg

tgatca
Sites

0

2

0

1

6

6

1

0

1

0

11

0

2

0

1

0

0

0

0

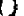

0 


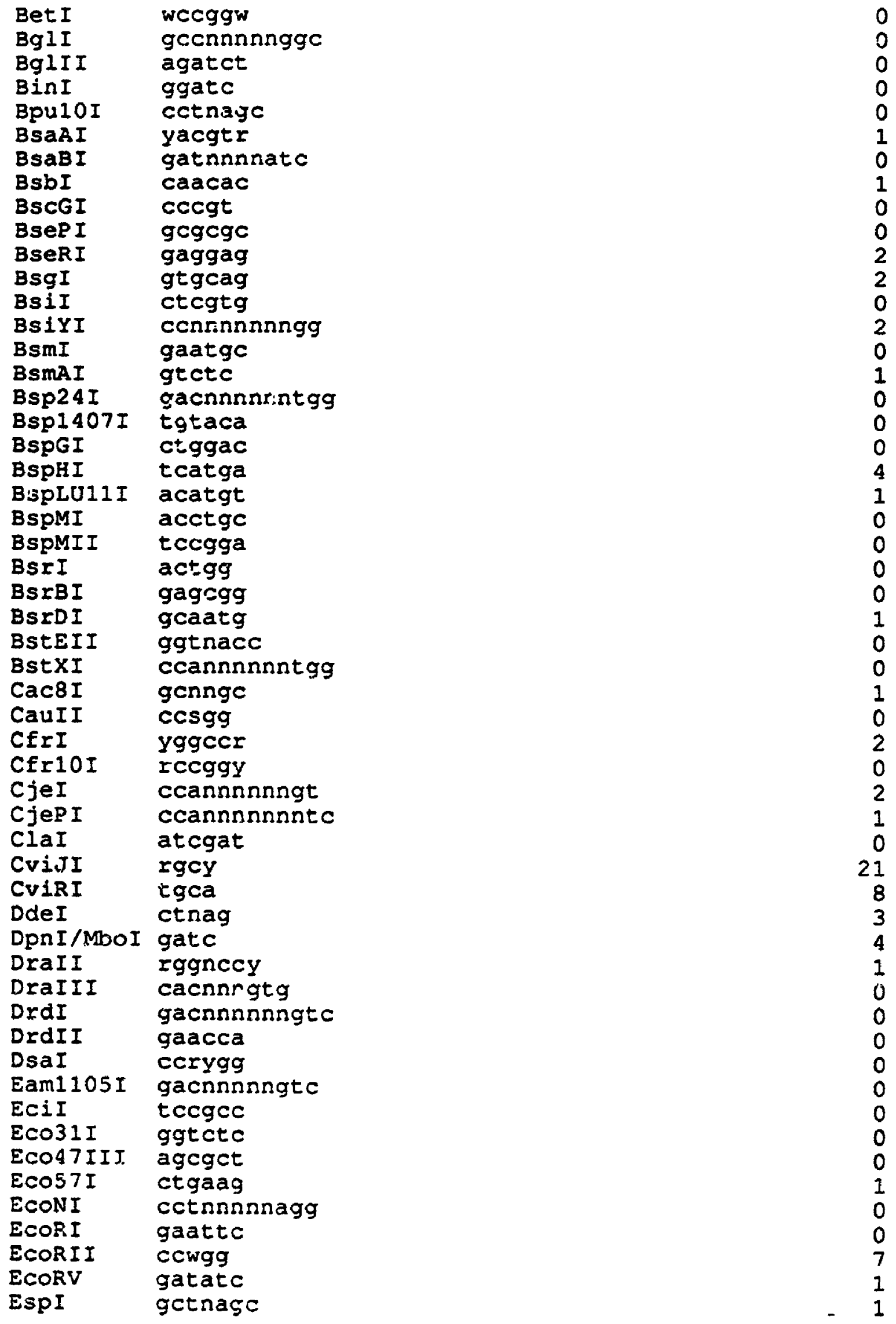




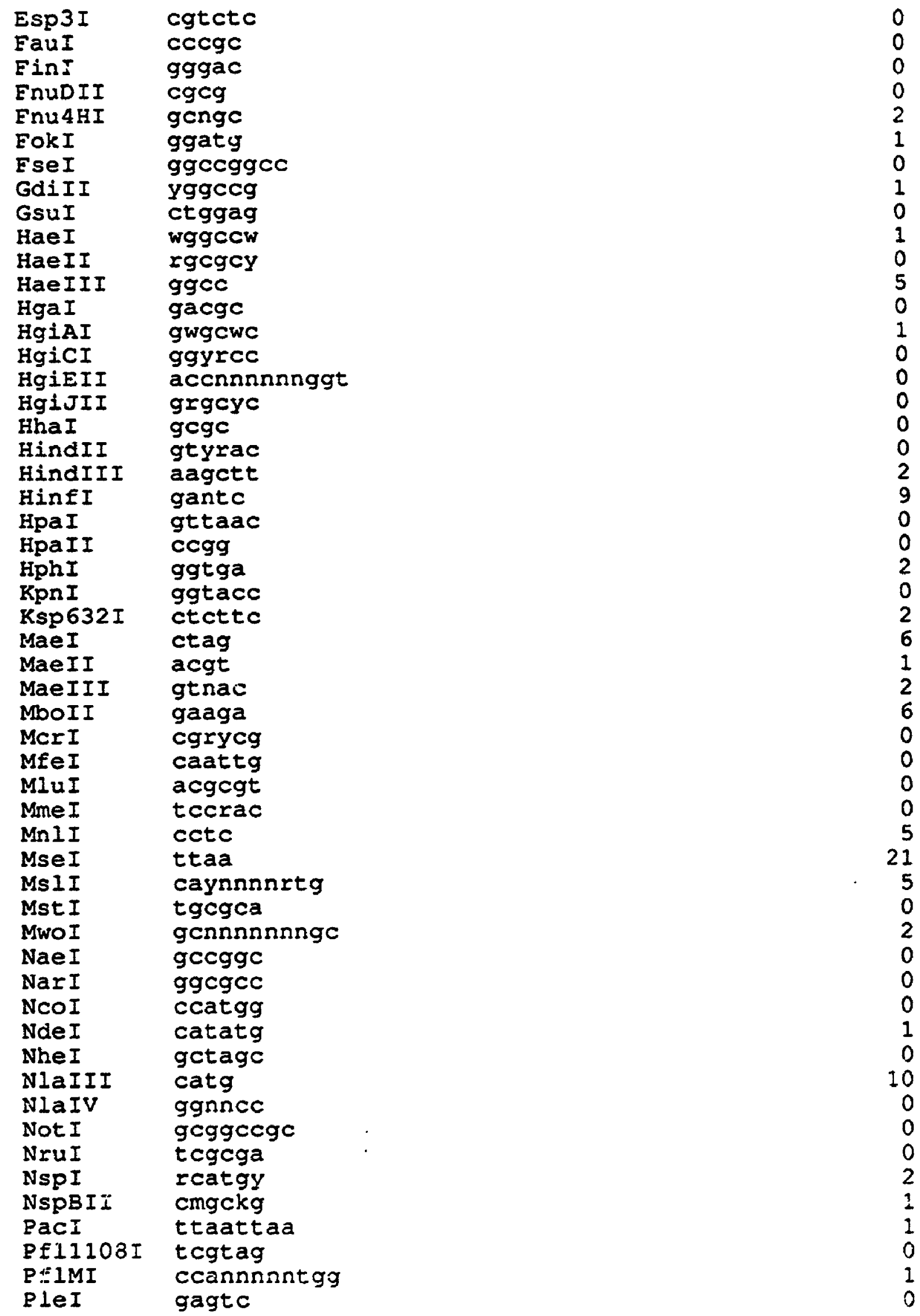




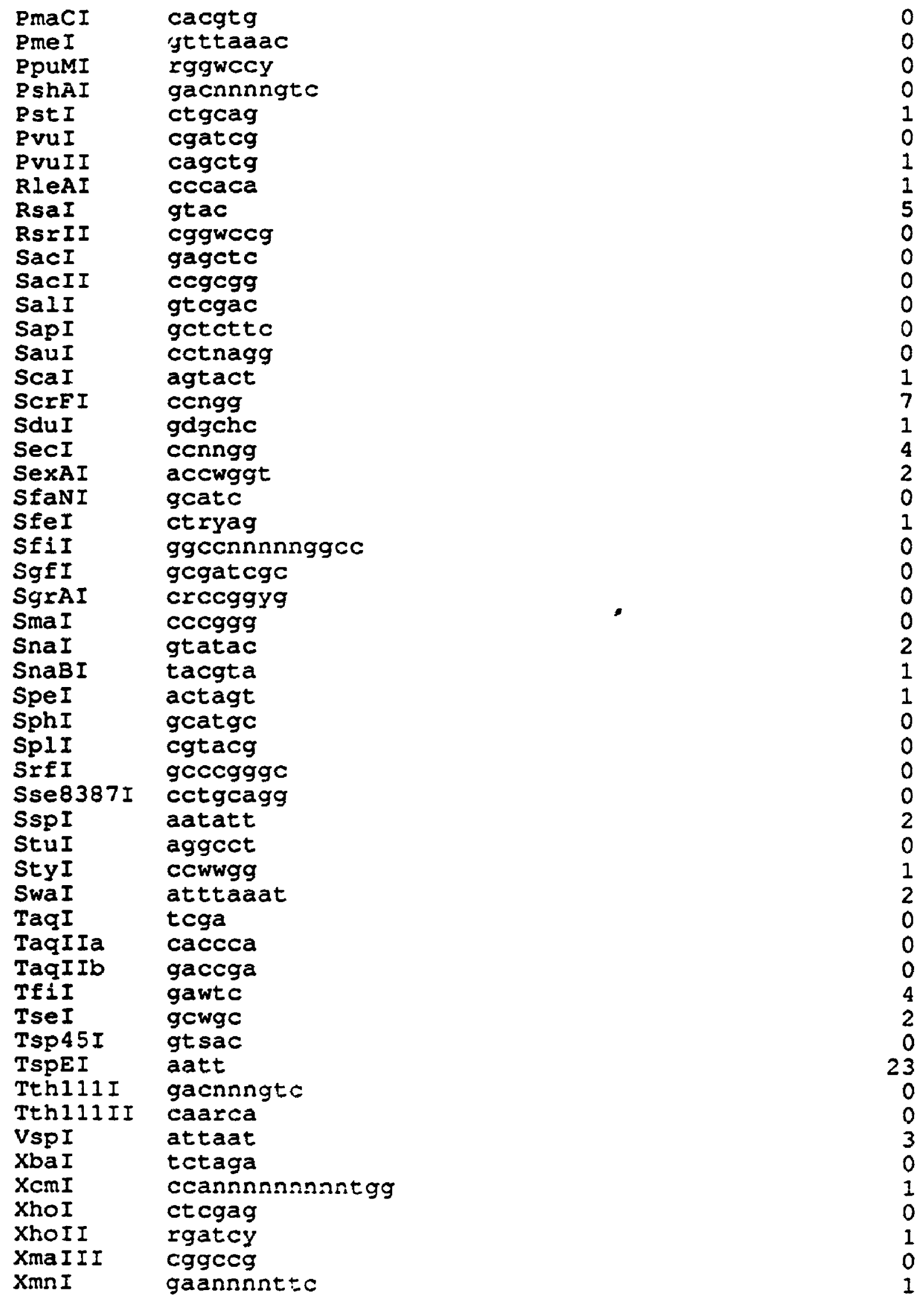


Total

265

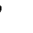




\section{Appendix G}

\section{Human Interferon-Alpha 5 Gene}

\section{G.1 Nucleotide Sequence}

The genomic DNA sequence of the human interferon-alpha 5 gene was retrieved from the Genbank sequence file hsifnas (Henco et. al., 1985) (Bowden et. al., 1993).

1 cttaatccgg gactgaat:id attctatttt acattctatt acgctgcttt taaagcatta

61 aagaagtaca atattctctc tcgataatgg gtactgtaat gtatatacat cagccaacac

121 atagtatatc tgtgttatta aaatttaatg ggattttag ttagaaaaa aattctaaa

181 aagcatatgt ggcagagtga agatgaggta ataatgtaaa aataantaaa ctgagaaaca

241 ctcctgtaca tctatgtaga aagagcataa aagaaagcaa aaagagaagt agaaagtaac

301 acaaggcatt cagaaaatgg aaactcgtat gtgaccttt taagatctgt gcacaaaaca

361 aggtcttcag agaagagcce aaggttcagg gtcactcaat ctcaaragcc cagaagcatc

421 tgcaacctcc ccaatggect tgcccttgt tttactgatg gecctggtgg tgetcaactg

481 caagtcaatc tgttctctgg gctgtgatct gcctcagacc cacagcctga gtaacaggag

541 gacttgatg ataatggcic aaatgggaag aatctctcct ttctcctgcc tgaaggacag

601 acatgactt ggattcctc aggaggagtt tgatggcaac cagttccaga aggctcaagc

661 catctctgtc ctccatgaga tgatccagca gaccttcaat ctcttcagca caaaggactc

721 atctgctact tgggatgaga cacttctaga caaattctac actgaacttt accagcagct

781 gaatgacctg gaagcctgta tgatgcagga ggttggagtg gaagacactc ctctgatgaa

841 tgtggactct atcetgactg tgagaaaata cttcaaaga atcaccctct atctgacaga

901 gaagaaatac agcccttgtg catgggaggt tgtcagagca gaaatcatga gatccttctc

561 ttatcagca aacttgcaag aaagattaag gaggaaggaa tgaaactgg ttcaacatcg

1021 aaatgattct cattgactag tacaccattt cacacttctt gagttetgcc gtttcaaata

1081 ttaatttctg ctatatccat gacttgagtt gaatcaaaat tttcaaacgt ttcacacgtg

1141 ttaagcaaca cttctttage tccacaggga caaaatcttt acagatgatc atgccaatct

1201 atctattcta tctatttatc tatctgtctg tcttctatct aatctatta aatatttatt

1261 tatttataag atttaaatta tttaaatta tgtttgttca ggtaatatta catccacctt

1321 tactttgtgg ctaatataat aaaatatgtt ctttatgttt tgtcaactga ttatttgct

1381 ttgttcatta gattttact attaattgtt tgttattct ttaaaatgaa actccaagcc

1441 tgattgtata acttgattaa aacagatgg tacag 


\section{G.2 Restriction Endonuclease Sites}

The human interferon-alpha 5 gene restriction endonuclease recognition sites are arranged by ascending sequence location.

\begin{tabular}{|c|c|}
\hline Name & site \\
\hline MseI & 2 \\
\hline HpaII & 7 \\
\hline CauII & 7 \\
\hline SCrFI & 7 \\
\hline FinI & 9 \\
\hline$X m n I$ & 15 \\
\hline ApoI & 19 \\
\hline TSPEI & 20 \\
\hline TseI & 43 \\
\hline FnU4HI & 43 \\
\hline MwoI & 46 \\
\hline AhaIII & 49 \\
\hline MseI & 50 \\
\hline MseI & 58 \\
\hline RsaI & 66 \\
\hline SspI & 70 \\
\hline Taq I & 81 \\
\hline RsaI & 91 \\
\hline CviJI & 112 \\
\hline BsbI & 115 \\
\hline MseI & 138 \\
\hline ApoI & 141 \\
\hline TSPEI & 142 \\
\hline MseI & 145 \\
\hline ApoI & 170 \\
\hline TSPEI & 171 \\
\hline MwoI & 183 \\
\hline NdeI & 184 \\
\hline MboII & 199 \\
\hline DdeI & 231 \\
\hline Bspl407I & 245 \\
\hline RsaI & 246 \\
\hline MaeIII & 296 \\
\hline Tsp45I & 331 \\
\hline MaeIII & 331 \\
\hline MseI & 340 \\
\hline BglII & 343 \\
\hline XhoII & 343 \\
\hline DpnI/MboI & 344 \\
\hline Apal I & 349 \\
\hline HgiAI & 349 \\
\hline SduI & \\
\hline
\end{tabular}




\begin{tabular}{|c|c|}
\hline 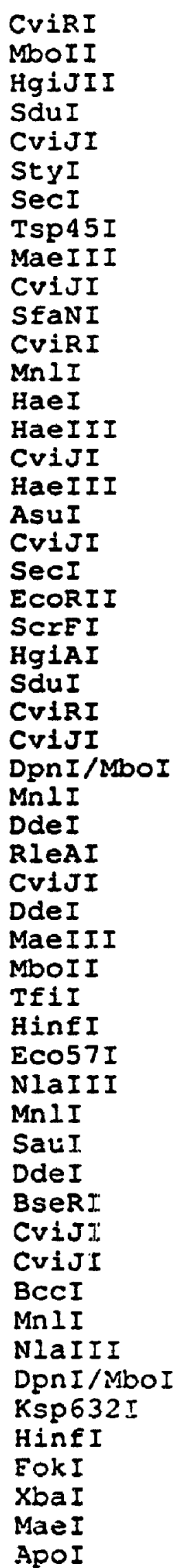 & $\begin{array}{l}350 \\
372 \\
375 \\
375 \\
376 \\
379 \\
379 \\
391 \\
391 \\
407 \\
416 \\
421 \\
426 \\
435 \\
436 \\
436 \\
460 \\
460 \\
460 \\
462 \\
463 \\
463 \\
470 \\
470 \\
479 \\
500 \\
506 \\
512 \\
513 \\
519 \\
524 \\
527 \\
531 \\
567 \\
570 \\
570 \\
590 \\
602 \\
617 \\
617 \\
618 \\
623 \\
652 \\
658 \\
660 \\
670 \\
674 \\
682 \\
701 \\
716 \\
733\end{array}$ \\
\hline
\end{tabular}




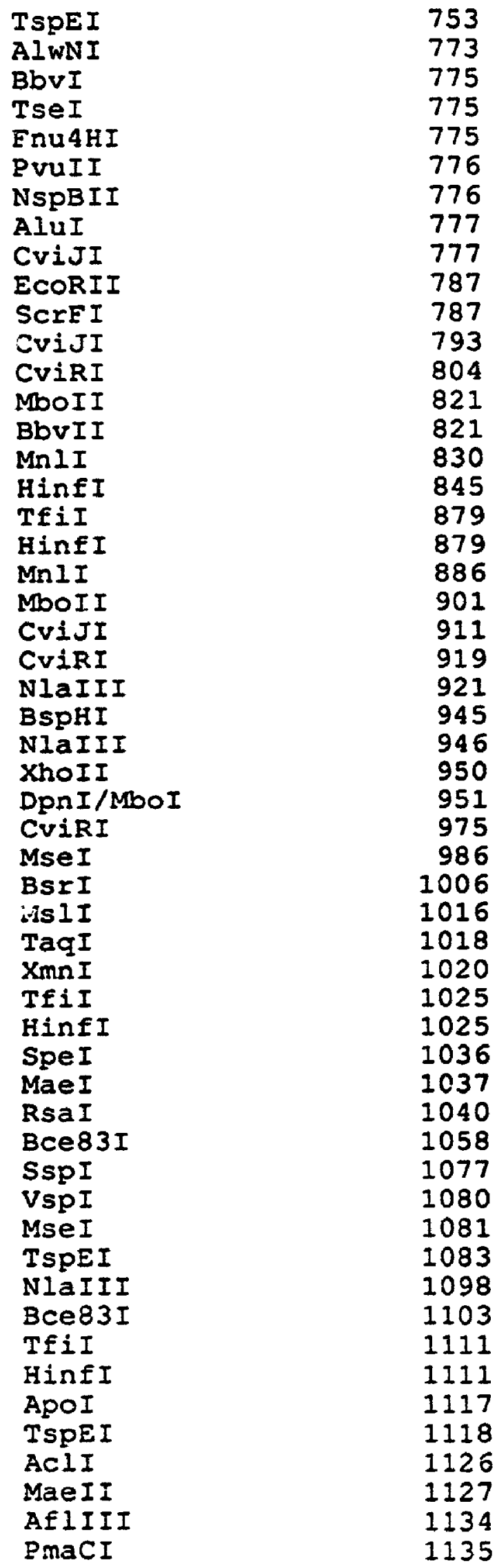




$\begin{array}{ll}\text { BsaAI } & 1135 \\ \text { MaeII } & 1136 \\ \text { AfIIII } & 1136 \\ \text { MseI } & 1141 \\ \text { BsbI } & 1146 \\ \text { AluI } & 1158 \\ \text { CviJI } & 1158 \\ \text { EinI } & 1167 \\ \text { BcII } & 1186 \\ \text { DpnI/MboI } & 1187 \\ \text { NIaIII } & 1190 \\ \text { SwaI } & 1246 \\ \text { AhaIII } & 1247 \\ \text { MseI } & 1248 \\ \text { SspI } & 1251 \\ \text { SwaI } & 1271 \\ \text { AhaIII } & 1272 \\ \text { MseI } & 1273 \\ \text { TspEI } & 1276 \\ \text { AhaIII } & 1282 \\ \text { MseI } & 1283 \\ \text { TspeI } & 1286 \\ \text { SspI } & 1304 \\ \text { CviJI } & 1329 \\ \text { HindII } & 1362 \\ \text { VspI } & 1401 \\ \text { MseI } & 1402 \\ \text { TspEI } & 1404 \\ \text { AhaIII } & 1420 \\ \text { MseI } & 1421 \\ \text { CviJI } & 1437 \\ \text { MseI } & 1457 \\ \text { RsaI } & 1470\end{array}$

The human interferon-alpha 5 gene restriction endonuclease recognition sites are arranged by enzyme.

$\begin{array}{lr}\text { Name } & \text { Site } \\ \text { AclI } & 1126 \\ \text { AfIIII } & 1134 \\ \text { AfIIII } & 1136 \\ \text { AhaIII } & 49 \\ \text { AhaIII } & 1247 \\ \text { AhaIII } & 1272 \\ \text { AhaIII } & 1282 \\ \text { AhaIII } & 1420 \\ \text { AluI } & 777 \\ \text { AluI } & 1158 \\ \text { Al:-NI } & 773\end{array}$




\begin{tabular}{|c|c|}
\hline $\begin{array}{l}\text { ApaII } \\
\text { ApoI } \\
\text { ApoI } \\
\text { ApoI } \\
\text { ApoI } \\
\text { ApoI } \\
\text { AsuI } \\
\text { BbvI } \\
\text { BbvII } \\
\text { BccI } \\
\text { Bce83I } \\
\text { Bce83I } \\
\text { BclI } \\
\text { BglII } \\
\text { BsaAI } \\
\text { BsbI } \\
\text { BsbI } \\
\text { BseRI } \\
\text { BspI407I } \\
\text { BspHI } \\
\text { BsrI } \\
\text { CauII } \\
\text { CviJI } \\
\text { CviJI } \\
\text { CviJI } \\
\text { CviJI } \\
\text { CviJI } \\
\text { CviJI } \\
\text { CviJI } \\
\text { CviJI } \\
\text { CviJI } \\
\text { CviJI } \\
\text { CviJI } \\
\text { CviJI } \\
\text { CviJI } \\
\text { CviJI } \\
\text { CviJI } \\
\text { CviRI } \\
\text { CviRI } \\
\text { CviRI } \\
\text { CviRI } \\
\text { CviRI } \\
\text { CviRI } \\
\text { DdeI } \\
\text { DdeI } \\
\text { DdeI } \\
\text { DdeI } \\
\text { DpnI/MboI } \\
\text { DpnI/MboI } \\
\text { DpnI/MboI } \\
\text { DpnI/MboI } \\
\text { DpnI/MboI } \\
\text { Eco5II } \\
\text { EcoRII }\end{array}$ & 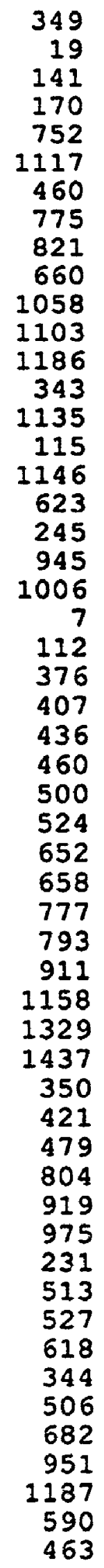 \\
\hline
\end{tabular}




\begin{tabular}{|c|c|}
\hline 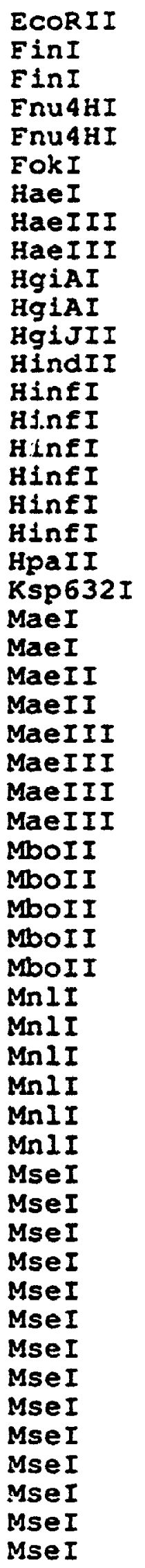 & $\begin{array}{r}787 \\
9 \\
1167 \\
43 \\
775 \\
733 \\
435 \\
436 \\
460 \\
349 \\
470 \\
375 \\
1362 \\
570 \\
716 \\
845 \\
879 \\
1025 \\
1111 \\
7 \\
701 \\
746 \\
1037 \\
1127 \\
1136 \\
296 \\
331 \\
391 \\
531 \\
199 \\
372 \\
567 \\
821 \\
901 \\
426 \\
512 \\
617 \\
670 \\
830 \\
886 \\
2 \\
50 \\
58 \\
138 \\
145 \\
340 \\
986 \\
1081 \\
1141 \\
1248 \\
1273 \\
1283 \\
1402 \\
1421\end{array}$ \\
\hline
\end{tabular}




$\begin{array}{lr}\text { MseI } & 1457 \\ \text { MsII } & 1016 \\ \text { MwoI } & 46 \\ \text { MwoI } & 183 \\ \text { NdeI } & 184 \\ \text { NlaIII } & 602 \\ \text { NIaIII } & 674 \\ \text { NlaIII } & 921 \\ \text { NlaIII } & 946 \\ \text { NlaIII } & 1098 \\ \text { NlaIII } & 1190 \\ \text { NspBII } & 776 \\ \text { PmaCI } & 1135 \\ \text { PvuII } & 776 \\ \text { RIeAI } & 519 \\ \text { RsaI } & 66 \\ \text { RsaI } & 91 \\ \text { RsaI } & 246 \\ \text { RsaI } & 1040 \\ \text { RsaI } & 1470 \\ \text { SauI } & 617 \\ \text { ScrEI } & 7 \\ \text { ScrII } & 710 \\ \text { ScrEI } & 1083 \\ \text { SduI } & 118 \\ \text { SduI } & \end{array}$




$\begin{array}{lr}\text { TspEI } & 1276 \\ \text { TspEI } & 1286 \\ \text { TspEI } & 1404 \\ \text { VspI } & 1080 \\ \text { VspI } & 1401 \\ \text { XbaI } & 745 \\ \text { XhoII } & 343 \\ \text { XhoII } & 950 \\ \text { XmnI } & 15 \\ \text { XmnI } & 1020\end{array}$

The human interferon-alpha 5 gene restriction endonuclease recognition sites are summarized by enzyme.

$\begin{array}{ll}\text { Name } & \text { Pattern } \\ \text { AatII } & \text { gacgtc } \\ \text { AccI } & \text { gtmkac } \\ \text { AcII } & \text { ccgc } \\ \text { AcII } & \text { aacgtt } \\ \text { AcyI } & \text { grcgyc } \\ \text { AfIII } & \text { cttaag } \\ \text { AfIIII } & \text { acrygt } \\ \text { AgeI } & \text { accggt } \\ \text { AhaIII } & \text { tttaaa } \\ \text { AluI } & \text { agct } \\ \text { AlwNI } & \text { cagnnnctg } \\ \text { ApaI } & \text { gggccc } \\ \text { ApaBI } & \text { gcannnnntgc } \\ \text { ApaII } & \text { gtgcac } \\ \text { ApoI } & \text { raatty } \\ \text { AscI } & \text { ggcgcgcc } \\ \text { AsuI } & \text { ggncc } \\ \text { AsuII } & \text { ttcgaa } \\ \text { AvaI } & \text { cycgrg } \\ \text { AvaII } & \text { ggwcc } \\ \text { AvaIII } & \text { atgcat } \\ \text { AvIII } & \text { cctagg } \\ \text { BaeI } & \text { acnnnngtayc } \\ \text { BalI } & \text { tggcca } \\ \text { BamiI } & \text { ggatcc } \\ \text { BbvI } & \text { gcagc } \\ \text { BbvII } & \text { gaagac } \\ \text { BccI } & \text { ccatc } \\ \text { Bce83I } & \text { cttgag } \\ \text { BcefI } & \text { acggc } \\ \text { BcgI } & \text { gcannnnnntcg } \\ \text { BcII } & \text { tgatca } \\ \text { BetI } & \text { wccggw } \\ \text { BglI } & \text { gccnnnnnggc } \\ & \end{array}$




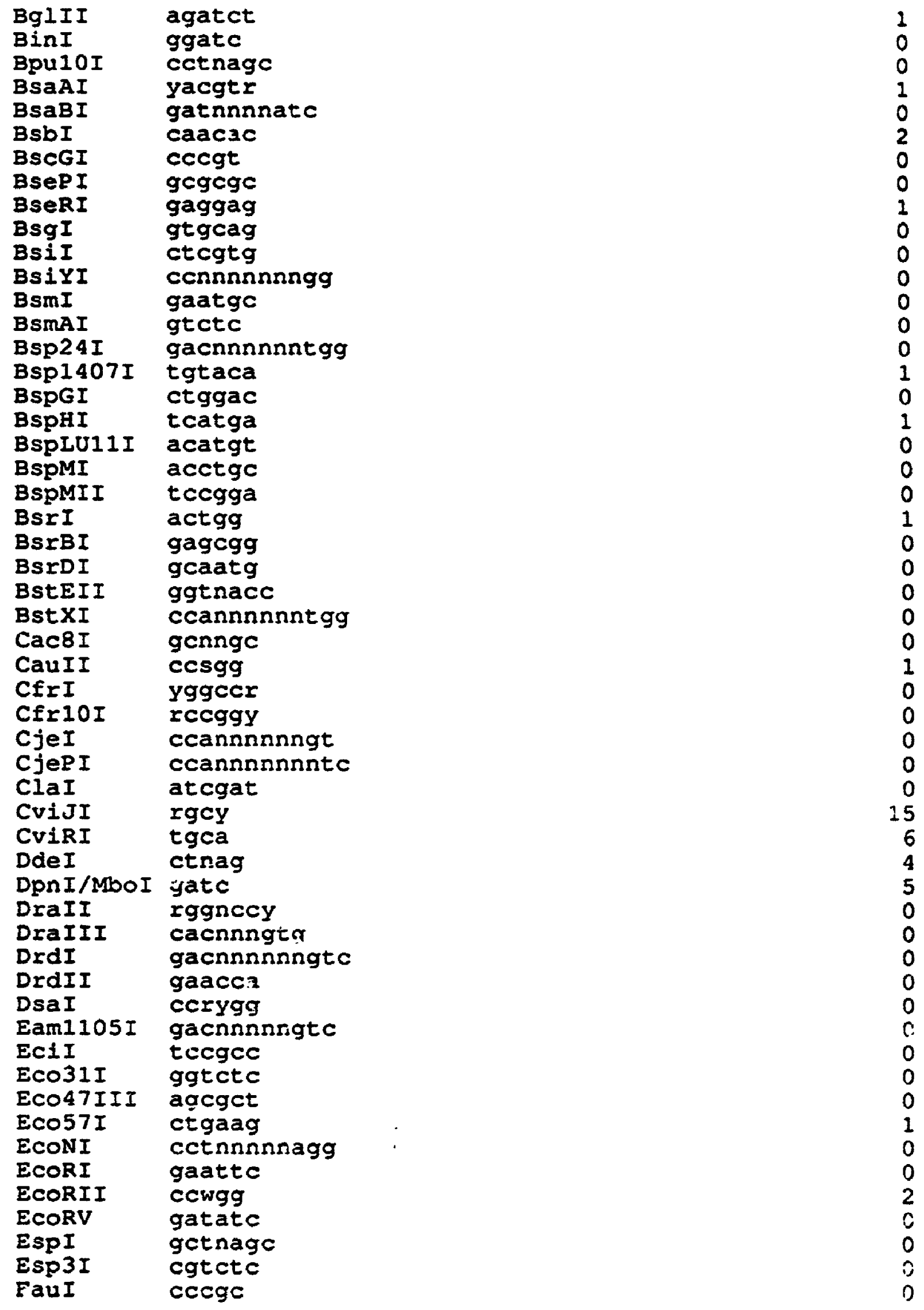




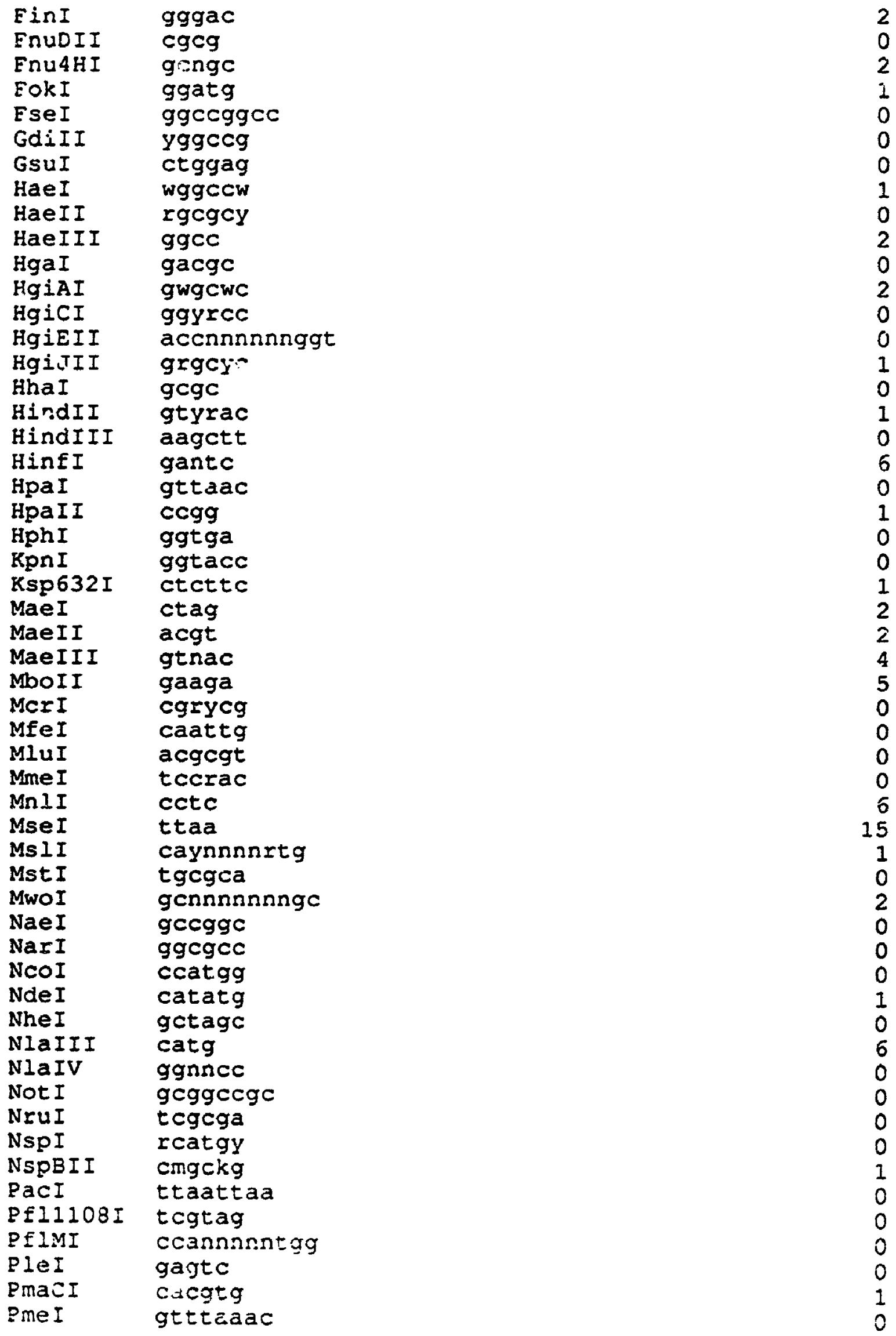




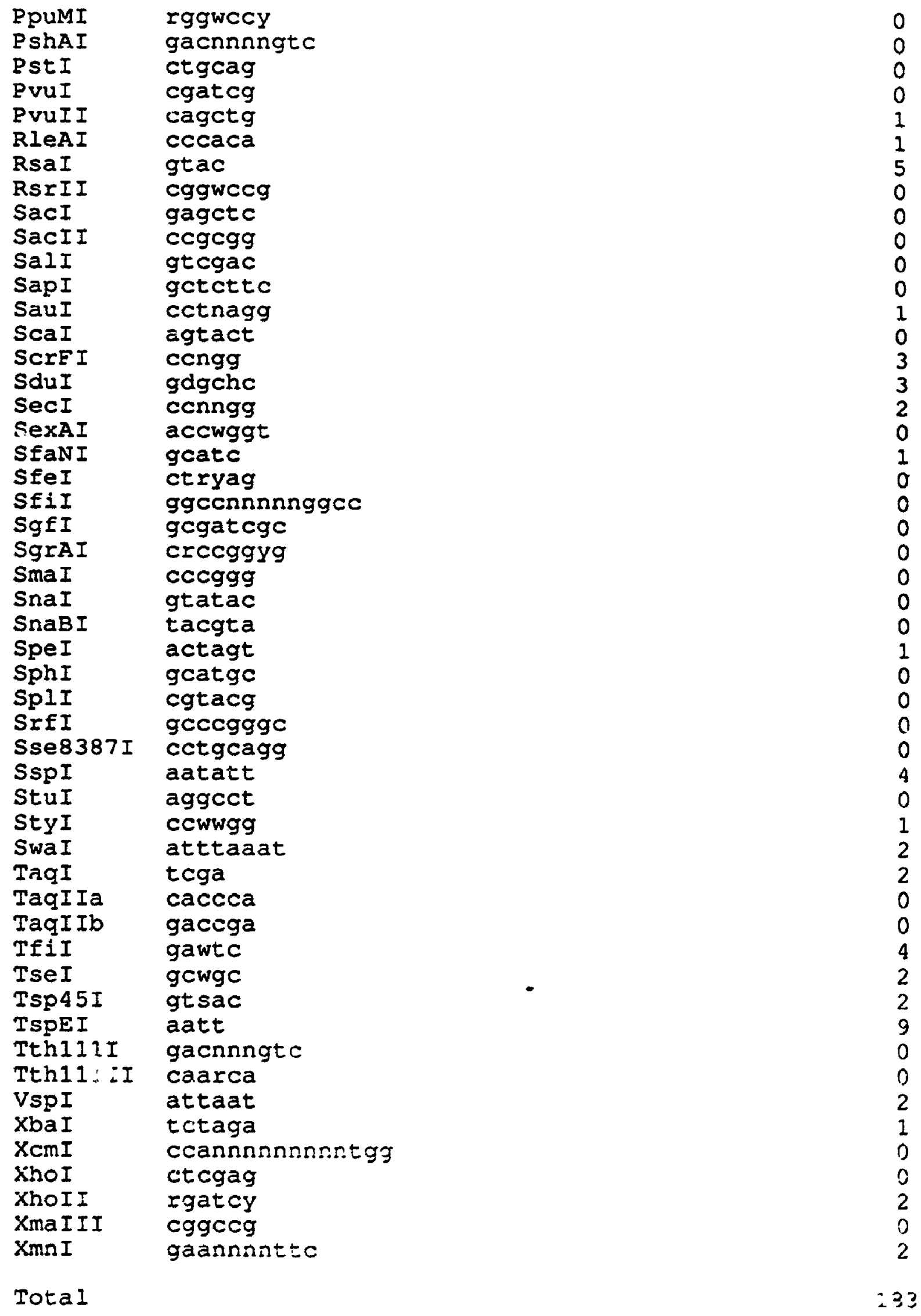




\title{
Appendix $\mathbf{H}$
}

\section{Human Interferon-Alpha 6 Gene}

\author{
H.1 Nucleotide Sequence
}

The genomic DNA sequence of the human interferon-alpha 6 gene was retrieved

from the Genbank sequence file hsifna6 (Henco et. al., 1985) (Benson et. al., 1993).

1 atgctcagag ttaagttaan tgtcaaatt traaaantt aactttagt taaattactt.

61 tgccattect aacagatcaa gaaataataa angtanactt tatgctttt ctttagtgt

121 gcaagtagac atacatacag tacataagga gatatacaat atatttgtta ttaaaatta

181 ataaaaatt aattcaatt aagaaaaaaa ttcttaaaa gactctggag gcaggcaaga

241 gaagggagtg tcaataatga ccacatacac acacaaacac acacacaaaa ctggttgaaa

301 aactactcta tacccatgta gagagtaaat aaatgaaagc aaaatcagac gtagaaagta

361 auttctgaaa atggaaacta gtatgttccc tattaagac ctacacataa agcauggtct

421 tcagagaacc tagagetgaa ggttcagagt cacccatctc aacaagtcca acagcatctg

481 caacatctac aatggctttg cctttgctt tactgatgge cctggtggtg ctcagctgca

541 agtcaagctg ctctctggac tgtgatctge ctcagaccca cagcctgggt cacaggagga

601 ccatgatgct cctggcacaa atgaggagaa tctctcttt ctcctgtctg aaggacagac

661 atgacttcag attccccag gaggagttg atggcazcca gttccagaag gctgaagcca

721 tctctgtcct ccatgaggtg attcagcaga cettcaatct cttcagcaca aaggactcat

781 ctgttgettg ggatgagagg cttctagaca aactctatac tgaactttac cagcagctga

841 atgacctgga agcctgtgtg atgcaggagg tgtgggtggg agggactccc ctgatgaatg

901 aggactccat cctggetgtg agaaaanct tccaaagaat cactctctac ctgacagaga

961 aaa gtacag cecttgtgec tgggaggttg tcagagcaga aatcatgaga tccttctctt

1021 catcaagaaa cttgcaagaa aggttaagga ggaaggaata agacctgatc cancacagaa

1081 acgactccca ttgacgacta caccagcttg cacttcatg atctgccatt ttaaagactc

1141 ttgttctge tataaccata ccatgagttg aatcaaacge gtcaagtatt ttcaagtgtg

1201 ttaagcaaca tcgtgttcag ttgcacagga actagtccet tacagatgac taagctgatg

1261 gatctatta tctatttatt aaatgtatat ttatttgaca ataatattta atttttgce

1321 catataagtg cataaaatgt ataccttat attgttgata acataacaaa atatatttc

1381 tatgtttagt cgatgtatta tttgtitag ttattaaat atttactata gaaaacttct

1441 tggattrgtt tattctctaa ggaganacac caagcegatt ctccatcatg attaaaaat

1501 gtatggtica attcattgat tcattgttat tatactcaag ttat 


\section{H.2 Restriction Endonuclease Sites}

The human interferon-alpha 6 gene restriction endonuclease recognition sites are arranged by ascending sequence location.

$\begin{array}{lr}\text { Name } & \text { Site } \\ \text { DdeI } & \\ \text { MseI } & 41 \\ \text { MseI } & 11 \\ \text { ApoI } & 16 \\ \text { TspEI } & 25 \\ \text { AnaIII } & 26 \\ \text { MseI } & 30 \\ \text { ApoI } & 31 \\ \text { TspEI } & 35 \\ \text { MseI } & 36 \\ \text { MseI } & 39 \\ \text { TspEI } & 50 \\ \text { DpnI/MoI } & 53 \\ \text { CviRI } & 75 \\ \text { AccI } & 120 \\ \text { RsaI } & 125 \\ \text { MseI } & 140 \\ \text { ApoI } & 171 \\ \text { TspEI } & 174 \\ \text { MseI } & 175 \\ \text { ApoI } & 178 \\ \text { TspEI } & 185 \\ \text { MseI } & 186 \\ \text { TspEI } & 189 \\ \text { TspEI } & 191 \\ \text { MseI } & 197 \\ \text { ApoI } & 199 \\ \text { TspEI } & 208 \\ \text { MseI } & 209 \\ \text { HinfI } & 215 \\ \text { GsuI } & 221 \\ \text { Cac8I } & 225 \\ \text { TthIIIII } & 231 \\ \text { BsII } & 274 \\ \text { NlaIII } & 290 \\ \text { MaeII } & 315 \\ \text { ApoI } & 349 \\ \text { TspEI } & 360 \\ \text { SpeI } & 361 \\ \text { MaeI } & 377 \\ \text { MseI } & 378 \\ \text { MaeI } & 394 \\ & 430\end{array}$




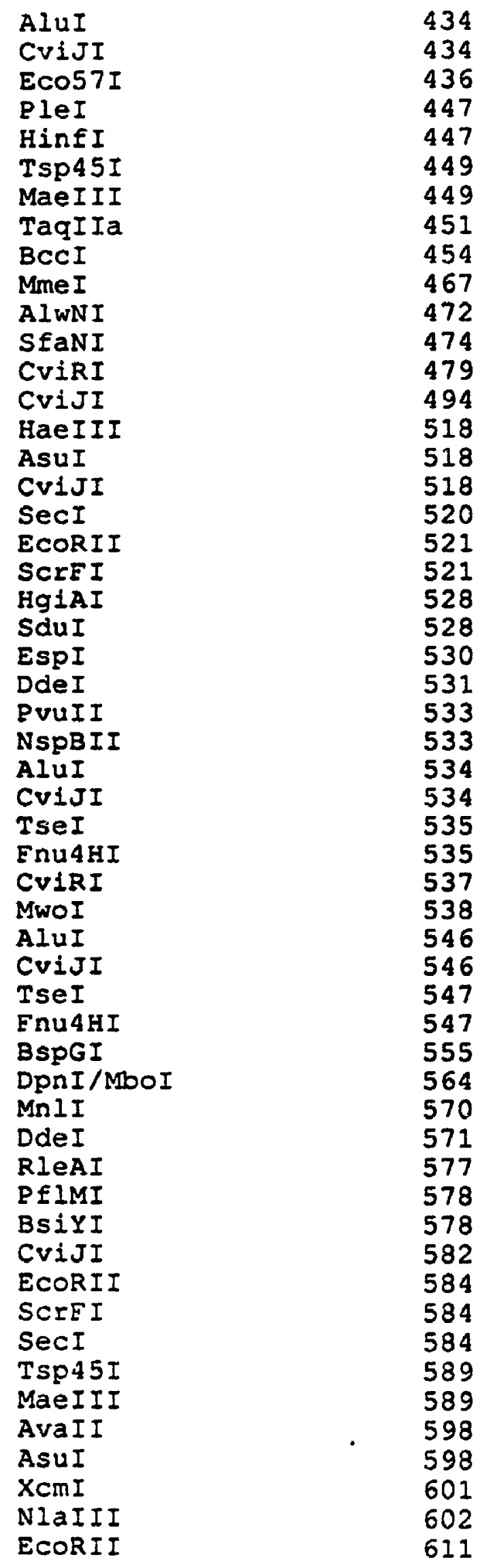




\begin{tabular}{|c|c|}
\hline 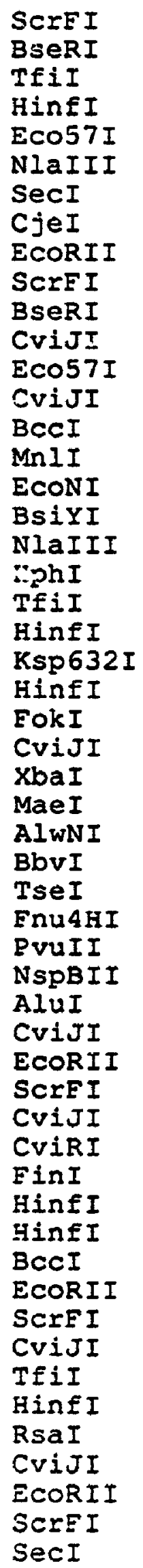 & $\begin{array}{l}611 \\
623 \\
628 \\
628 \\
648 \\
660 \\
676 \\
677 \\
677 \\
677 \\
681 \\
710 \\
712 \\
716 \\
718 \\
728 \\
728 \\
728 \\
732 \\
737 \\
740 \\
740 \\
759 \\
774 \\
791 \\
799 \\
803 \\
804 \\
831 \\
833 \\
833 \\
833 \\
834 \\
834 \\
835 \\
835 \\
845 \\
845 \\
851 \\
862 \\
882 \\
884 \\
903 \\
907 \\
911 \\
911 \\
914 \\
937 \\
937 \\
965 \\
969\end{array}$ \\
\hline
\end{tabular}




\begin{tabular}{|c|c|}
\hline $\begin{array}{l}\text { BspHI } \\
\text { NlaII } \\
\text { XhoII } \\
\text { DpnI/MboI } \\
\text { Ksp632I } \\
\text { CviRI } \\
\text { MseI } \\
\text { DpnI/MooI } \\
\text { MmeI } \\
\text { BsbI } \\
\text { HinfI } \\
\text { AluI } \\
\text { CviJI } \\
\text { Cac8I } \\
\text { CviRI } \\
\text { MsII } \\
\text { BspHI } \\
\text { NlaIII } \\
\text { DpnI/MboI } \\
\text { AhaIII } \\
\text { MseI } \\
\text { HinfI } \\
\text { NlaIII } \\
\text { TfiI } \\
\text { HinfI } \\
\text { MluI } \\
\text { AflIII } \\
\text { EnuDII } \\
\text { MseI } \\
\text { CviRI } \\
\text { SpeI } \\
\text { MaeI } \\
\text { DdeI } \\
\text { AluI } \\
\text { CviJI } \\
\text { BinI } \\
\text { XhoII } \\
\text { DpnI/MboI } \\
\text { MseI } \\
\text { SspI } \\
\text { MseI } \\
\text { TspEI } \\
\text { MsII } \\
\text { CviRI } \\
\text { SnaI } \\
\text { AccI } \\
\text { TaqI } \\
\text { MseI } \\
\text { SspI } \\
\text { SfeI } \\
\text { DdeI } \\
\text { CjePI } \\
\text { CviJI } \\
\text { TfiI } \\
\text { Mfing }\end{array}$ & $\begin{array}{l}1003 \\
1004 \\
1008 \\
1009 \\
1016 \\
1033 \\
1044 \\
1067 \\
1069 \\
1071 \\
1083 \\
1105 \\
1105 \\
1106 \\
1109 \\
1111 \\
1116 \\
1117 \\
1120 \\
1130 \\
1131 \\
1136 \\
1162 \\
1170 \\
1170 \\
1179 \\
1177 \\
1178 \\
1201 \\
1222 \\
1231 \\
1232 \\
1250 \\
1253 \\
1253 \\
1260 \\
1260 \\
1261 \\
1279 \\
1303 \\
1308 \\
1310 \\
1321 \\
1329 \\
1339 \\
1339 \\
1390 \\
1415 \\
1418 \\
1426 \\
1457 \\
1470 \\
1473 \\
1477\end{array}$ \\
\hline
\end{tabular}




$\begin{array}{ll}\text { HinfI } & 1477 \\ \text { MmeI } & 1482 \\ \text { NlaIII } & 1487 \\ \text { AhaIII } & 1492 \\ \text { MseI } & 1493 \\ \text { TspEI } & 1510 \\ \text { TfiI } & 1518 \\ \text { HinfI } & 1518\end{array}$

The human interferon-alpha 6 gene restriction endonuclease recognition sites are arranged by enzyme.

Name

ACCI

ACCI

AEIIII

AhaIII

AhaIII

AhaIII

AluI

AluI

AluI

AluI

AluI

AluI

AlwNI

AlwNI

ApoI

ApoI

ApoI

ApoI

ApoI

ApoI

AsuI

AsuI

AvaII

BbvI

BCCI

BCCI

BCCI

BinI

BsbI

BseRI

BSeRI

BSIYI

BSIYI

BspGI

BspHI

BspHI
Site

125

1339

1177

30

1130

1492

434

534

546

835

1105

1253

472

831

25

35

174

185

208

360

518

598

598

833

454

718

907

1260

1071

623

681

578

728

555

1003

1116 


\begin{tabular}{|c|c|}
\hline 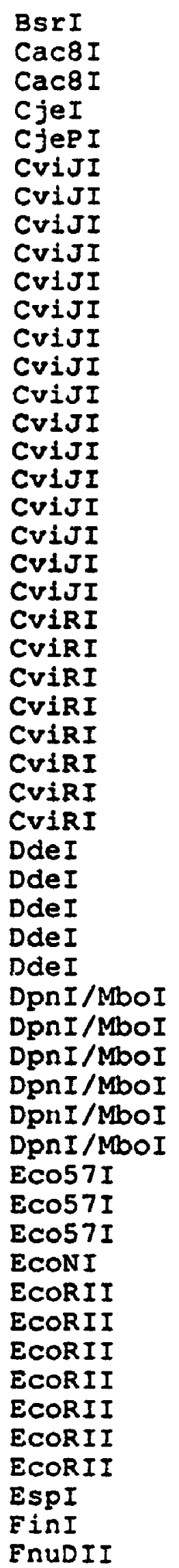 & 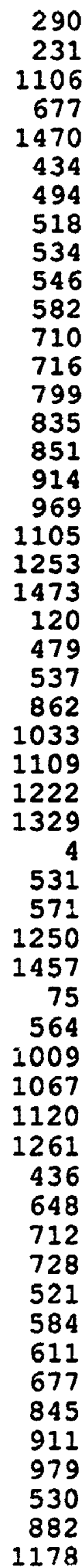 \\
\hline
\end{tabular}




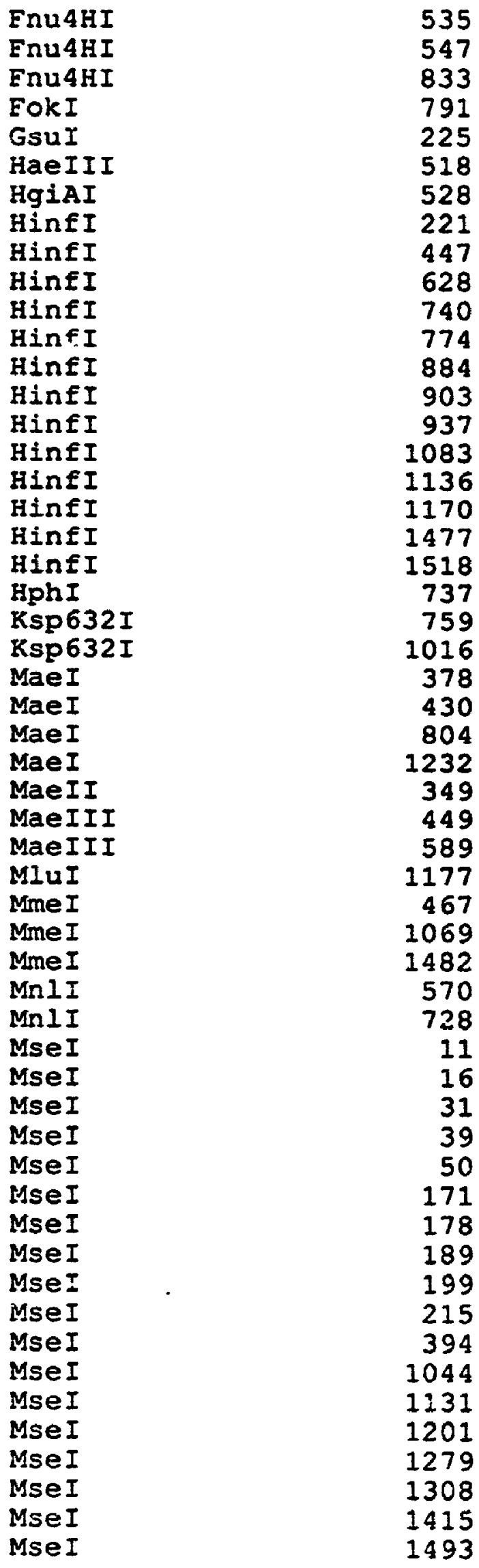




\begin{tabular}{|c|c|}
\hline $\begin{array}{l}\text { MsII } \\
\text { MsII } \\
\text { MwoI } \\
\text { NlaIII } \\
\text { NlaIII } \\
\text { NlaIII } \\
\text { NlaIII } \\
\text { NlaIII } \\
\text { NlaIII } \\
\text { NlaIII } \\
\text { NlaIII } \\
\text { NspBII } \\
\text { NspBII } \\
\text { PfIMI } \\
\text { PIeI } \\
\text { PvuII } \\
\text { PvuII } \\
\text { RleAI } \\
\text { RsaI } \\
\text { RsaI } \\
\text { ScrFI } \\
\text { ScrFI } \\
\text { ScrFI } \\
\text { ScrEI } \\
\text { ScrEI } \\
\text { ScrEI } \\
\text { ScrEI } \\
\text { SduI } \\
\text { SecI } \\
\text { SecI } \\
\text { SecI } \\
\text { SecI } \\
\text { SfaNI } \\
\text { SfeI } \\
\text { SnaI } \\
\text { SpeI } \\
\text { SpeI } \\
\text { SspI } \\
\text { SspI } \\
\text { TaqI } \\
\text { TaqIIa } \\
\text { TfiI } \\
\text { TfiI } \\
\text { TfiI } \\
\text { TfiI } \\
\text { TfiI } \\
\text { TfiI } \\
\text { TseI } \\
\text { TseI } \\
\text { TseI } \\
\text { Tsp45I } \\
\text { Tsp45I } \\
\text { TspEI } \\
\text { TspEI }\end{array}$ & $\begin{array}{r}1111 \\
1321 \\
538 \\
315 \\
602 \\
660 \\
732 \\
1004 \\
1117 \\
1162 \\
1487 \\
533 \\
834 \\
578 \\
447 \\
533 \\
834 \\
577 \\
140 \\
965 \\
521 \\
584 \\
611 \\
677 \\
845 \\
911 \\
979 \\
528 \\
520 \\
584 \\
676 \\
979 \\
474 \\
1426 \\
1339 \\
377 \\
1231 \\
1303 \\
1418 \\
1390 \\
451 \\
628 \\
740 \\
937 \\
1170 \\
1477 \\
1518 \\
535 \\
547 \\
833 \\
449 \\
589 \\
26 \\
36\end{array}$ \\
\hline
\end{tabular}




$\begin{array}{lr}\text { TSPEI } & 53 \\ \text { TSPEI } & 175 \\ \text { TSPEI } & 186 \\ \text { TSPEI } & 191 \\ \text { TSPEI } & 197 \\ \text { TSPEI } & 209 \\ \text { TSpEI } & 361 \\ \text { TSPEI } & 1310 \\ \text { TSPEI } & 1510 \\ \text { TthIIIII } & 274 \\ \text { XbaI } & 803 \\ \text { XCmI } & 601 \\ \text { XhoII } & 1008 \\ \text { XhoII } & 1260\end{array}$

The human interferon-alpha 6 gene restriction endonuclease recognition sites are summarized by enzyme.

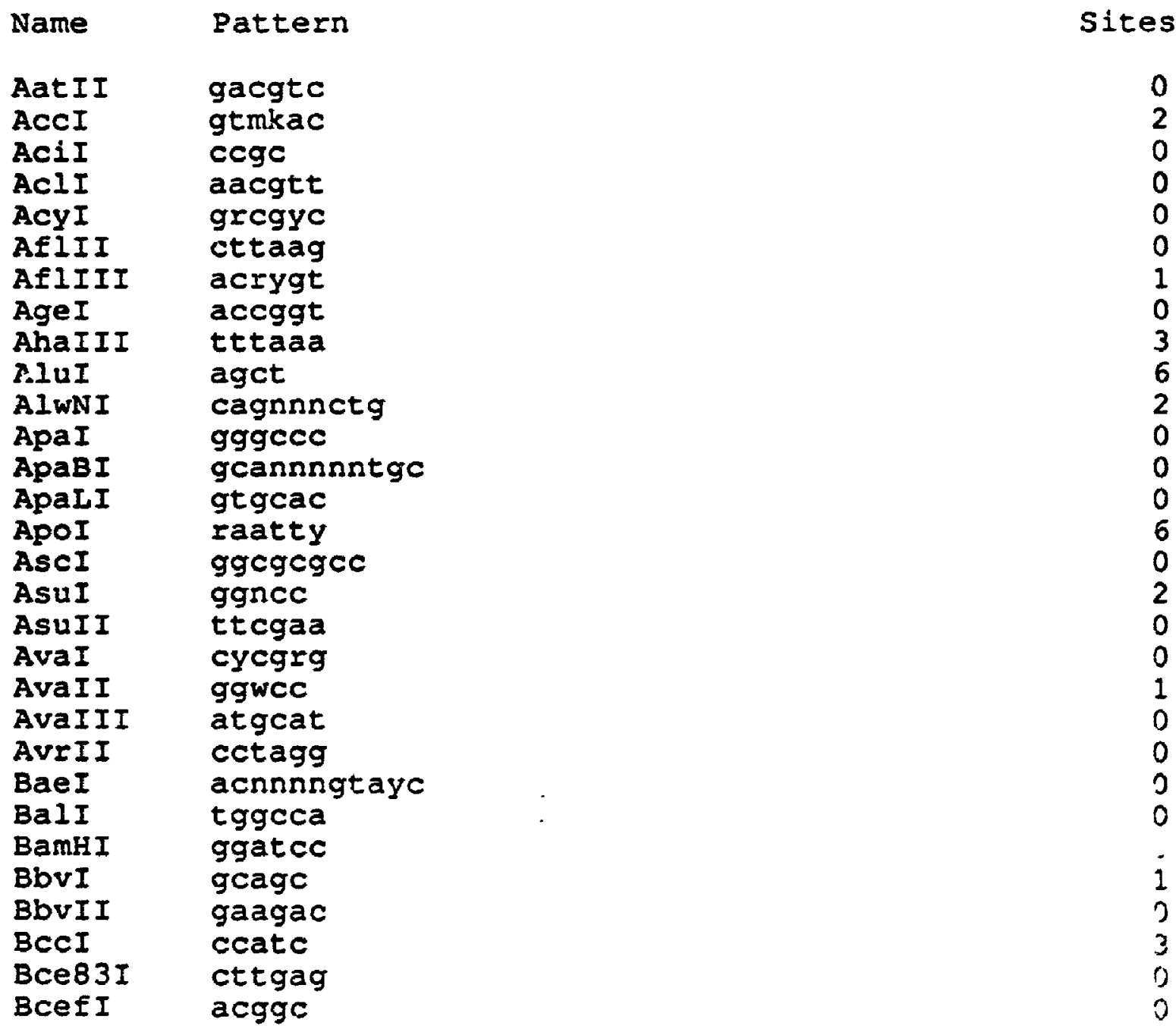




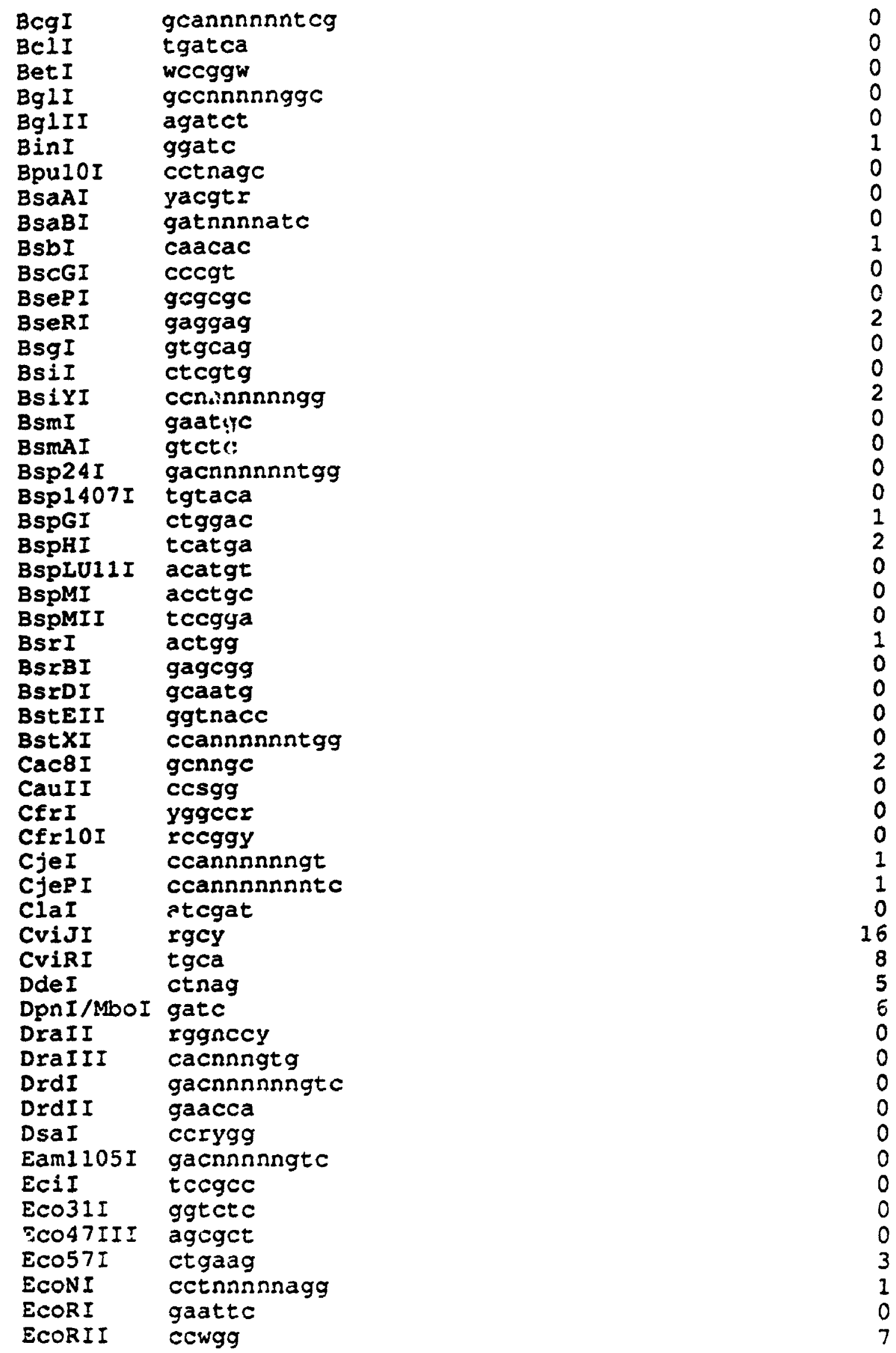




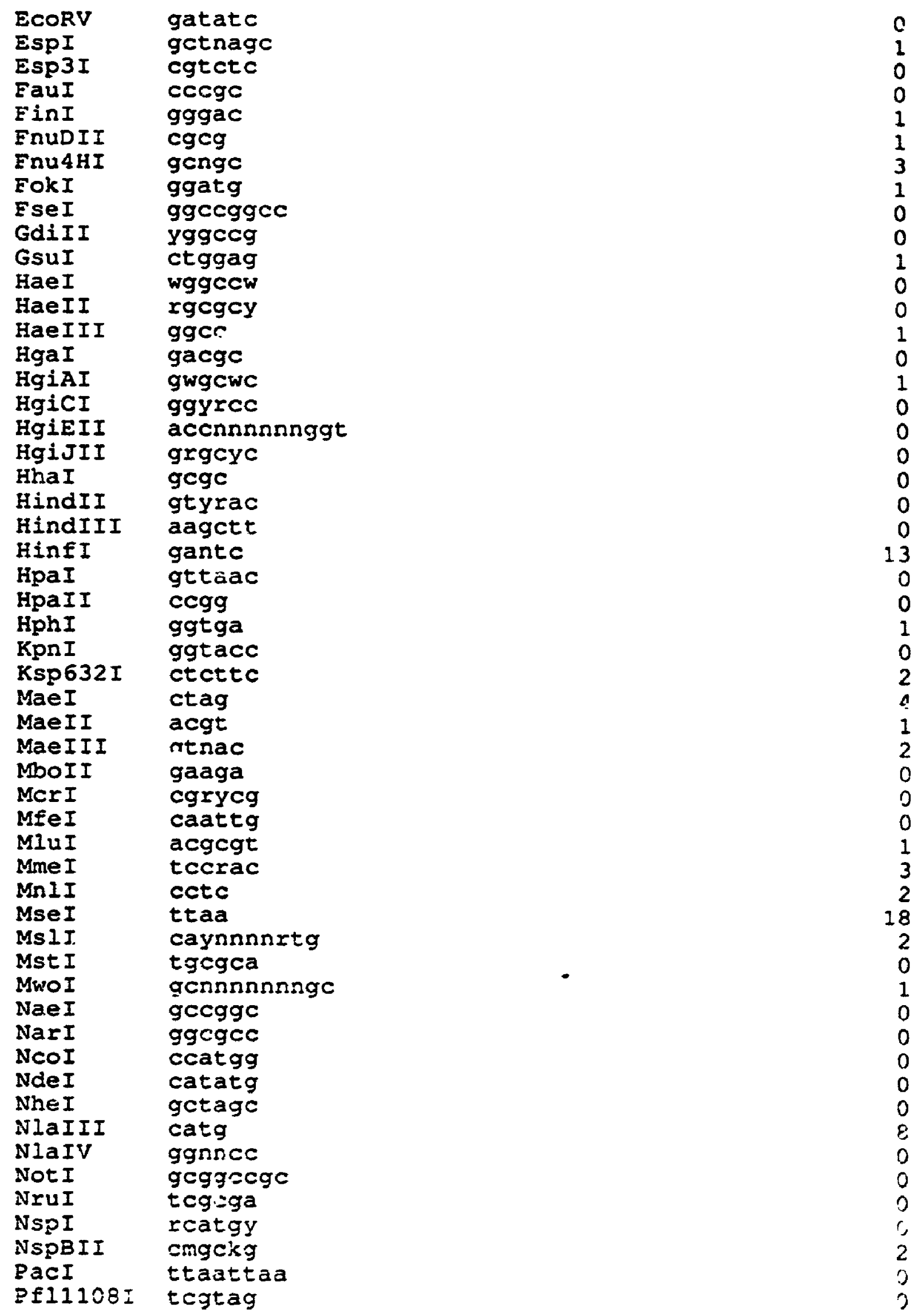




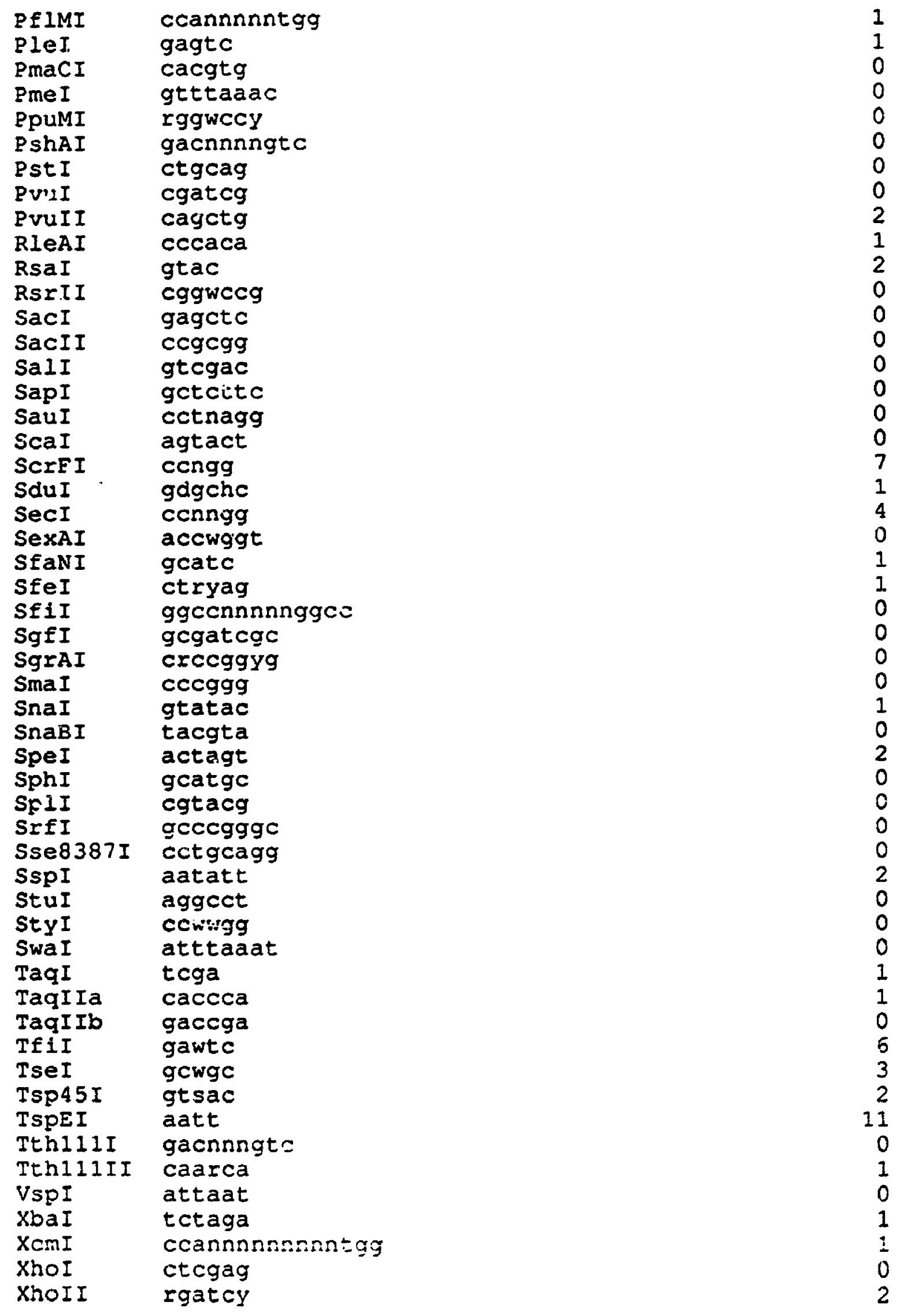


Xma I I I

cggecg

0

$X m n I$

gaannnntto

0

Totàl

212 


\section{Appendix I}

\section{Human Interferon-Alpha 7 Gene}

\section{I.1 Nucleotide Sequence}

The genomic DNA sequence of the human interferon-alpha 7 gene was retrieved

from the Genbank file hsifna7 (Henco et. al., 1985) (Benson et. al., 1993).

1 cacatcagta cttatgtcaa gtgctgaaaa gaaaaangtg tggcaatat ctggatgaat

61 actgcagcta gtgaagttta caaattatt tctcatataa agcaaantc aaagcttcat

121 atactatgag aaaattttt taaaattgat tcatatttct agcagıtttg aatgattagg

181 tatgtaatta cattcatatt aatgtgtatt atacagattt ttatttgca tatgtaattt

241 gaaacsonaa aattracatg aacaaattac attaaaagtt attccacaaa tatacttatc

301 taattaaact tagatttaa tagctttaa acttagattt tagttaact tttctgtcat

361 tettaactta ctttgaataa aaagagcaaa cttcatact ttatctgtg aagtagaggt

421 atatgtagaa tacctaaata gatatgccaa atctgtgtta ttaaaatttc atgaacattt

481 caattagaaa aaaataccat aaaaggcttt gagtgcaggg gaaaaacagg caatgatgaa

541 aaaaaaaatg aaaaacgtat ttaaacacat ggagagagtg cataaagaaa gcaaaaacag

601 agatagaaag taaaactagg geatttagaa aatggaaatt agtatgttca ctatttaaga

661 cctatgcaca gagcaaagtc tccagaaaac ctagaggcca cggttcaagt tacccacctc

721 aggtagccta gtgatatttg caaaatccca atggeccggt cctttctt actgatggtc

781 gtgetggtac tcagctacaa atccatctge tctctggget gtgatctgec tcagacceac

841 agctgegta ataggaggge cttgatactc ctggcacaaa tgggaagaat ctctccttc

901 tcctgettga aggacagaca tgaattcaga ttcccagagg aggagttga tggccaccag

961 ttccagaaga ctcaagccat ctctgtcctc catgagatga tcragcagac cttcaatctc

1021 ttcagcacag aggactcatc tgctgettgg gaacagagcc tcctagaaaa attrccact

1081 gaacttrace agcaactgaa tgacctggaa gcatgtgtga tacaggaggt tggggtggaa

1141 gagacteccc tgatgaatga ggacttcatc ctggctgtga ggaaatactt ccaaagaatc

1201 actcttatc taatggagaa gaaatacage cettgtgcct gggaggttgt cagagcagaa

1261 atcatgagat ccttctctt ttcaacaanc ttgaaaaaag gattaaggag gaaggattga

1321 aaactggttc atcatggaan tgattctcat tgactaatgc atcatctcac acttcatga

1381 gttctccat ttcaaagact cacttctata accaccacaa gttgaatcaa aatttccaaa

1441 tgtttcagg agtgttanga ageatcgtgt ttacetgtge aggcactagt ccttacaga

$150 !$ tgaccattct gatgtctcet ttcatctatt tatttaaata tttatttatt taactatttt

1561 tattatttaa attantttt atgtaatatc atatgtacet ttacattgtg gttaatgtaa

1621 caaatatgtt cttcatatt agccaatata ttaatttcet tttcattaa attttacta

1681 tacauaattt cttgtgattg tttantttt aagattaaat gecaagcctg actgtataac

1741 ctgacttana aatagatgat ttaagtaagt tacctatcat aatttattc aagttataga

1801 aanatatatt ttctatace aggttatctg ttgccttcat gatatuaacg tgas ontaaa

1861 anatacagtt ctigttcter tgtatctttg attttgtca ggaangaaat ctaa:s:sacaa

1921 taataatgct gaattaatat cggttatact auctgctgta atgtgaggaa gtaaaaaaa

1981 atg 


\section{I.2 Restriction Endonuclease Sites}

The human interferon-alpha 7 gene restriction endonuclease recognition sites are arranged by ascending sequence location.

\begin{tabular}{|c|c|}
\hline Name & Site \\
\hline ScaI & 7 \\
\hline RsaI & 8 \\
\hline FokI & 53 \\
\hline PstI & 62 \\
\hline SfeI & 62 \\
\hline CviRI & 63 \\
\hline BbvI & 64 \\
\hline TseI & 64 \\
\hline Enu $4 \mathrm{HI}$ & 6 \\
\hline AluI & 6 \\
\hline CviJI & 6 \\
\hline Mae I & 68 \\
\hline TSPEI & 8 . \\
\hline ApoI & 10 \\
\hline TSPEI & 10 \\
\hline HindIII & 11 \\
\hline AluI & 11 \\
\hline CVIJI & 11 \\
\hline ApoI & 132 \\
\hline TSPEI & \\
\hline AhaIII & 13 \\
\hline MseI & \\
\hline TSPEI & \\
\hline TEII & \\
\hline HinfI & \\
\hline MaeI & \\
\hline TSPEI & \\
\hline Ms1I & \\
\hline VspI & \\
\hline MséI & \\
\hline CviRI & \\
\hline NdeI & \\
\hline TspEI & \\
\hline ApoI & \\
\hline TSpEI & \\
\hline NlaIII & \\
\hline TSPEI & \\
\hline MseI & \\
\hline TspEI & \\
\hline MseI & \\
\hline DdeI & \\
\hline MseI & \\
\hline
\end{tabular}




\begin{tabular}{|c|c|}
\hline $\begin{array}{l}\text { AluI } \\
\text { CviJI } \\
\text { AhaIII } \\
\text { MseI } \\
\text { DdeI } \\
\text { MseI } \\
\text { MseI } \\
\text { MseI } \\
\text { ApoI } \\
\text { TspEI } \\
\text { BspHI } \\
\text { NlaIII } \\
\text { TspEI } \\
\text { CviJI } \\
\text { MwoI } \\
\text { BsgI } \\
\text { CviRI } \\
\text { BsrDI } \\
\text { MaeII } \\
\text { AhaIII } \\
\text { MseI } \\
\text { NlaIII } \\
\text { CviRI } \\
\text { MaeI } \\
\text { TspEI } \\
\text { MseI } \\
\text { CviRI } \\
\text { BsmAI } \\
\text { MaeI } \\
\text { HaeI } \\
\text { HaeIII } \\
\text { CviJI } \\
\text { DsaI } \\
\text { SecI } \\
\text { MaeIII } \\
\text { BsiYI } \\
\text { CjeI } \\
\text { MnII } \\
\text { SauI } \\
\text { DdeI } \\
\text { CviJI } \\
\text { MaeI } \\
\text { CviRI } \\
\text { HaeIII } \\
\text { AsuI } \\
\text { CviJI } \\
\text { CauII } \\
\text { ScrEI } \\
\text { HpaII } \\
\text { AvaII } \\
\text { AsuI } \\
\text { RsaI } \\
\text { DdeI } \\
\text { AIUI }\end{array}$ & $\begin{array}{l}322 \\
322 \\
326 \\
327 \\
332 \\
345 \\
363 \\
461 \\
464 \\
465 \\
469 \\
470 \\
482 \\
505 \\
506 \\
513 \\
514 \\
530 \\
555 \\
560 \\
561 \\
568 \\
579 \\
616 \\
637 \\
655 \\
665 \\
678 \\
691 \\
695 \\
696 \\
696 \\
698 \\
698 \\
709 \\
713 \\
714 \\
717 \\
717 \\
718 \\
725 \\
728 \\
739 \\
753 \\
753 \\
753 \\
755 \\
755 \\
756 \\
758 \\
758 \\
787 \\
790 \\
793\end{array}$ \\
\hline
\end{tabular}




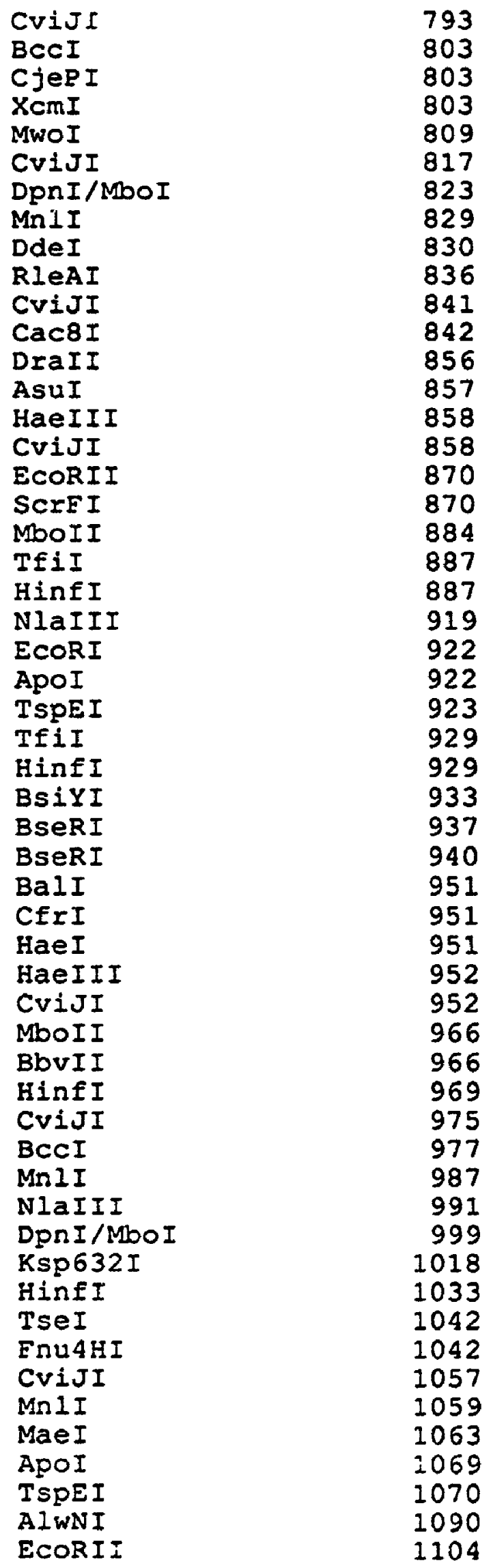




\begin{tabular}{|c|c|}
\hline $\begin{array}{l}\text { ScrEI } \\
\text { NspI } \\
\text { NlaIII } \\
\text { MooII } \\
\text { HinfI } \\
\text { EcoRII } \\
\text { ScrEI } \\
\text { CviJI } \\
\text { XmnI } \\
\text { TfiI } \\
\text { HinfI } \\
\text { MboII } \\
\text { CviJI } \\
\text { EcoRII } \\
\text { ScrEI } \\
\text { SecI } \\
\text { BspHI } \\
\text { NlaIII } \\
\text { XhoII } \\
\text { DpnI/MboI } \\
\text { MseI } \\
\text { BsrI } \\
\text { NlaIII } \\
\text { MslI } \\
\text { XmnI } \\
\text { TfiI } \\
\text { HinfI } \\
\text { AvaIII } \\
\text { CviRI } \\
\text { SEaNI } \\
\text { MslI } \\
\text { BspHI } \\
\text { NIaIII } \\
\text { HinfI } \\
\text { CjeI } \\
\text { TfiI } \\
\text { HinfI } \\
\text { ApoI } \\
\text { TspEI } \\
\text { CjePI } \\
\text { MseI } \\
\text { SfaNI } \\
\text { BsgI } \\
\text { CviRI } \\
\text { Cac8I } \\
\text { SpeI } \\
\text { MaeI } \\
\text { MsII } \\
\text { BsmAI } \\
\text { SwaI } \\
\text { AhaIII } \\
\text { MseI } \\
\text { SspI } \\
\text { MseI } \\
\text { Ming }\end{array}$ & $\begin{array}{l}1104 \\
1111 \\
1112 \\
1138 \\
1143 \\
1170 \\
1170 \\
1173 \\
1182 \\
1196 \\
1196 \\
1218 \\
1228 \\
1238 \\
1238 \\
1238 \\
1262 \\
1263 \\
1267 \\
1268 \\
1303 \\
1323 \\
1333 \\
1333 \\
1337 \\
1342 \\
1342 \\
1357 \\
1358 \\
1359 \\
1370 \\
1375 \\
1376 \\
1397 \\
1412 \\
1424 \\
1424 \\
1430 \\
1431 \\
1436 \\
1455 \\
1462 \\
1477 \\
1478 \\
1479 \\
1485 \\
1486 \\
1505 \\
1514 \\
1532 \\
1533 \\
1534 \\
1537 \\
1550\end{array}$ \\
\hline
\end{tabular}




\begin{tabular}{ll} 
SwaI & 1565 \\
AhaIII & 1566 \\
MseI & 1567 \\
TspEI & 1570 \\
NdeI & 1590 \\
RsaI & 1595 \\
MseI & 1612 \\
MaeIII & 1617 \\
CviJI & 1641 \\
VspI & 1650 \\
MseI & 1651 \\
TspEI & 1653 \\
MseI & 1667 \\
ApoI & 1669 \\
TspEI & 1670 \\
ApoI & 1685 \\
TspEI & 1686 \\
MseI & 1709 \\
MseI & 1715 \\
CviJI & 1725 \\
MseI & 1746 \\
MseI & 1761 \\
MaeIII & 1769 \\
TspEI & 1781 \\
SexAI & 1818 \\
EcoRII & 1819 \\
ScrEI & 1819 \\
BspHI & 1837 \\
NlaIII & 1838 \\
MaeII & 1848 \\
TspEI & 1932 \\
VspI & 1933 \\
MseI & 1934 \\
& \\
\hline sse &
\end{tabular}

The human interferon-alpha 7 gene restriction endonuclease recognition sites are arranged by enzyme.

Name

AhaIII

AhaIII

AhaIII

Aha III

AhaIII

AluI

AluI

AluI

AluI

AIWN I

ApoI site

139

326

560

1533

1566

66

113

322

793

1090

105 


\begin{tabular}{|c|c|}
\hline $\begin{array}{l}\text { ApoI } \\
\text { ApoI } \\
\text { ApoI } \\
\text { ApoI } \\
\text { ApoI } \\
\text { ApoI } \\
\text { ApoI } \\
\text { ApoI } \\
\text { AsuI } \\
\text { AsuI } \\
\text { AsuI } \\
\text { AvaII } \\
\text { AvaIII } \\
\text { BalI } \\
\text { BbvI } \\
\text { BbvII } \\
\text { BcCI } \\
\text { BcCI } \\
\text { BseRI } \\
\text { BseRI } \\
\text { BsgI } \\
\text { BsgI } \\
\text { BsiYI } \\
\text { BsiYI } \\
\text { BsmaI } \\
\text { BsmaI } \\
\text { BspHI } \\
\text { BspHI } \\
\text { BspHI } \\
\text { BspHI } \\
\text { BsrI } \\
\text { BsrDI } \\
\text { Cac8I } \\
\text { Cac8I } \\
\text { CauII } \\
\text { CErI } \\
\text { CjeI } \\
\text { CjeI } \\
\text { CjePI } \\
\text { CjePI } \\
\text { CviJI } \\
\text { CviJI } \\
\text { CviJI } \\
\text { CviJI } \\
\text { CviJI } \\
\text { CviJI } \\
\text { CviJI } \\
\text { CviJI } \\
\text { CviJI } \\
\text { CviJI } \\
\text { CviJI } \\
\text { CviJI } \\
\text { CviJI } \\
\text { CviJI }\end{array}$ & 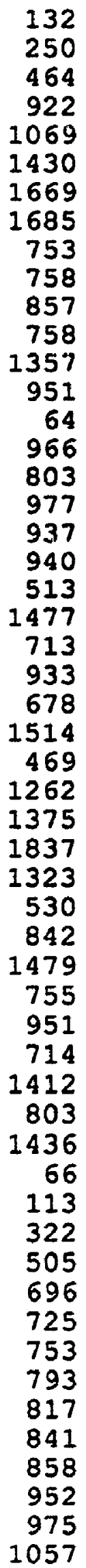 \\
\hline
\end{tabular}




\begin{tabular}{|c|c|}
\hline $\begin{array}{l}\text { CviJI } \\
\text { CviJI } \\
\text { CviJI } \\
\text { CviJI } \\
\text { CviRI } \\
\text { CviRI } \\
\text { CviRI } \\
\text { CviRI } \\
\text { CviRI } \\
\text { CviRI } \\
\text { CviRI } \\
\text { CviRI } \\
\text { DdeI } \\
\text { DdeI } \\
\text { DdeI } \\
\text { DdeI } \\
\text { DdeI } \\
\text { DpnI/MboI } \\
\text { DpnI/MboI } \\
\text { DpnI/MboI } \\
\text { DraII } \\
\text { DsaI } \\
\text { EcoRI } \\
\text { EcoRII } \\
\text { EcoRII } \\
\text { EcoRII } \\
\text { EcoRII } \\
\text { EcoRII } \\
\text { Enu4HI } \\
\text { Enu4HI } \\
\text { EokI } \\
\text { "aeI } \\
\text { HaeI } \\
\text { HaeIII } \\
\text { HaeIII } \\
\text { HaeIII } \\
\text { HaeITI } \\
\text { HindIII } \\
\text { HinfI } \\
\text { HinfI } \\
\text { HinfI } \\
\text { HinfI } \\
\text { HinfI } \\
\text { HinfI } \\
\text { HinfI } \\
\text { HinfI } \\
\text { HinfI } \\
\text { HinfI } \\
\text { HpaII } \\
\text { Ksp632I } \\
\text { MaeI } \\
\text { MaeI } \\
\text { MaeI } \\
\text { MaeI } \\
\text { Hading }\end{array}$ & $\begin{array}{r}1173 \\
1228 \\
1641 \\
1725 \\
63 \\
227 \\
514 \\
579 \\
665 \\
739 \\
1358 \\
1478 \\
309 \\
332 \\
718 \\
790 \\
830 \\
823 \\
999 \\
1268 \\
856 \\
698 \\
922 \\
870 \\
1104 \\
1170 \\
1238 \\
1819 \\
64 \\
1042 \\
53 \\
695 \\
951 \\
696 \\
753 \\
858 \\
952 \\
112 \\
148 \\
887 \\
929 \\
969 \\
1033 \\
1143 \\
1196 \\
1342 \\
1397 \\
1424 \\
756 \\
1018 \\
68 \\
159 \\
616 \\
691\end{array}$ \\
\hline
\end{tabular}




\begin{tabular}{|c|c|}
\hline $\begin{array}{l}\text { MaeI } \\
\text { MaeI } \\
\text { MaeI } \\
\text { MaeII } \\
\text { MaeII } \\
\text { MaeIII } \\
\text { MaeIII } \\
\text { MaeIII } \\
\text { MboII } \\
\text { MboII } \\
\text { MboII } \\
\text { MboII } \\
\text { MnII } \\
\text { MnII } \\
\text { MnII } \\
\text { MnII } \\
\text { MseI } \\
\text { MseI } \\
\text { MseI } \\
\text { MseI } \\
\text { MseI } \\
\text { MseI } \\
\text { MseI } \\
\text { MseI } \\
\text { MseI } \\
\text { MseI } \\
\text { MseI } \\
\text { MseI } \\
\text { MseI } \\
\text { MseI } \\
\text { MseI } \\
\text { MseI } \\
\text { MseI } \\
\text { MseI } \\
\text { MseI } \\
\text { MseI } \\
\text { MseI } \\
\text { MseI } \\
\text { MseI } \\
\text { MseI } \\
\text { MsII } \\
\text { MsII } \\
\text { MsII } \\
\text { MsII } \\
\text { MwoI } \\
\text { MwoI } \\
\text { NdeI } \\
\text { NdeI } \\
\text { NlaIII } \\
\text { NlaIII } \\
\text { NIaIII } \\
\text { NlaIII } \\
\text { NIaIII } \\
\text { NlaIII }\end{array}$ & $\begin{array}{r}728 \\
1063 \\
1486 \\
555 \\
1848 \\
709 \\
1617 \\
1769 \\
884 \\
966 \\
1138 \\
1218 \\
717 \\
829 \\
987 \\
1059 \\
140 \\
199 \\
272 \\
304 \\
317 \\
327 \\
345 \\
363 \\
461 \\
561 \\
65.5 \\
1303 \\
1455 \\
1534 \\
1550 \\
1567 \\
1612 \\
1651 \\
1667 \\
1709 \\
1715 \\
1746 \\
1761 \\
1934 \\
195 \\
1333 \\
1370 \\
1505 \\
506 \\
809 \\
229 \\
1590 \\
257 \\
4710 \\
568 \\
919 \\
991 \\
1112\end{array}$ \\
\hline
\end{tabular}




\begin{tabular}{|c|c|}
\hline $\begin{array}{l}\text { NlaIII } \\
\text { NlaIII } \\
\text { NlaIII } \\
\text { NlaIII } \\
\text { NspI } \\
\text { PstI } \\
\text { RleAI } \\
\text { RsaI } \\
\text { RsaI } \\
\text { RsaI } \\
\text { SauI } \\
\text { ScaI } \\
\text { ScrEI } \\
\text { ScrEI } \\
\text { ScrEI } \\
\text { ScrEI } \\
\text { ScrFI } \\
\text { ScrEI } \\
\text { SecI } \\
\text { SecI } \\
\text { SexAI } \\
\text { SfaNI } \\
\text { SfaNI } \\
\text { SfeI } \\
\text { SpeI } \\
\text { SspI } \\
\text { SwaI } \\
\text { SwaI } \\
\text { TEiI } \\
\text { TfiI } \\
\text { TfiI } \\
\text { TfiI } \\
\text { TfiI } \\
\text { TfiI } \\
\text { TseI } \\
\text { TseI } \\
\text { TspEI } \\
\text { TspEI } \\
\text { TspEI } \\
\text { TspEI } \\
\text { TspEI } \\
\text { TspEI } \\
\text { TspEI } \\
\text { TspEI } \\
\text { TspEI } \\
\text { TspEI } \\
\text { TspEI } \\
\text { TspEI } \\
\text { TspEI } \\
\text { TspEI } \\
\text { TspEI } \\
\text { TspEI } \\
\text { TspEI } \\
\text { TspEI }\end{array}$ & $\begin{array}{r}1263 \\
1333 \\
1376 \\
1838 \\
1111 \\
62 \\
836 \\
8 \\
787 \\
1595 \\
717 \\
7 \\
755 \\
870 \\
1104 \\
1170 \\
1238 \\
1819 \\
698 \\
1238 \\
1818 \\
1359 \\
1462 \\
62 \\
1485 \\
1537 \\
1532 \\
1565 \\
148 \\
887 \\
929 \\
1196 \\
1342 \\
1424 \\
64 \\
1042 \\
83 \\
106 \\
133 \\
144 \\
186 \\
236 \\
251 \\
265 \\
302\end{array}$ \\
\hline
\end{tabular}




$\begin{array}{lr}\text { TSPEI } & 1685 \\ \text { TSPEI } & 1781 \\ \text { TSpEI } & 1932 \\ \text { VspI } & 198 \\ \text { VspI } & 1650 \\ \text { VspI } & 1933 \\ \text { XcmI } & 803 \\ \text { XhoII } & 1267 \\ \text { XmnI } & 1182 \\ \text { XmnI } & 1337\end{array}$

The human interferon-alpha 7 gene restriction endonuclease recognition sites are summarized by enzyme.

\begin{tabular}{|c|c|c|}
\hline Name & Pattern & Sites \\
\hline AatII & gacgtc & 0 \\
\hline ACCI & gtmkac & 0 \\
\hline AciI & $\operatorname{cog} c$ & 0 \\
\hline ACII & aacgtt & 0 \\
\hline AcyI & grcgyc & 0 \\
\hline AfIII & cttaag & 0 \\
\hline AfIIII & acrygt & 0 \\
\hline AgeI & accggt & 0 \\
\hline AhaIII & tttaaa & 5 \\
\hline AluI & agct & 4 \\
\hline AlWNI & cagnnnctg & 1 \\
\hline ApaI & gggccc & 0 \\
\hline ApaBI & gcannnnntgc & c \\
\hline ApaL $\ddot{z}$ & gtgcac & 0 \\
\hline ApoI & raatty & 9 \\
\hline AscI & ggcgcgce & 0 \\
\hline $\begin{array}{l}\text { AsuI } \\
\text { AsuII }\end{array}$ & $\begin{array}{l}\text { ggnec } \\
\text { ttcaaa }\end{array}$ & $\begin{array}{l}3 \\
0\end{array}$ \\
\hline AvaI & cycgrg & 0 \\
\hline AvaII & ggwce & 1 \\
\hline AvaIII & atgcat & 1 \\
\hline AvrII & cctagg & 0 \\
\hline BaeI & acnnnngtayc & 0 \\
\hline BalI & tggcca & 1 \\
\hline BamkI & ggatcc & 0 \\
\hline BbvI & gcoge & 1 \\
\hline BbvII & gaagac & 1 \\
\hline BccI & ccatc & 2 \\
\hline Bce83I & cttgag & 0 \\
\hline BcefI & acgge & 0 \\
\hline$B c g I$ & gcannnanatcy & 0 \\
\hline$B \subset I I$ & tgatca & 0 \\
\hline BetI & wesggw & $\hat{\jmath}$ \\
\hline BglI & gccnnnnngge & \\
\hline
\end{tabular}




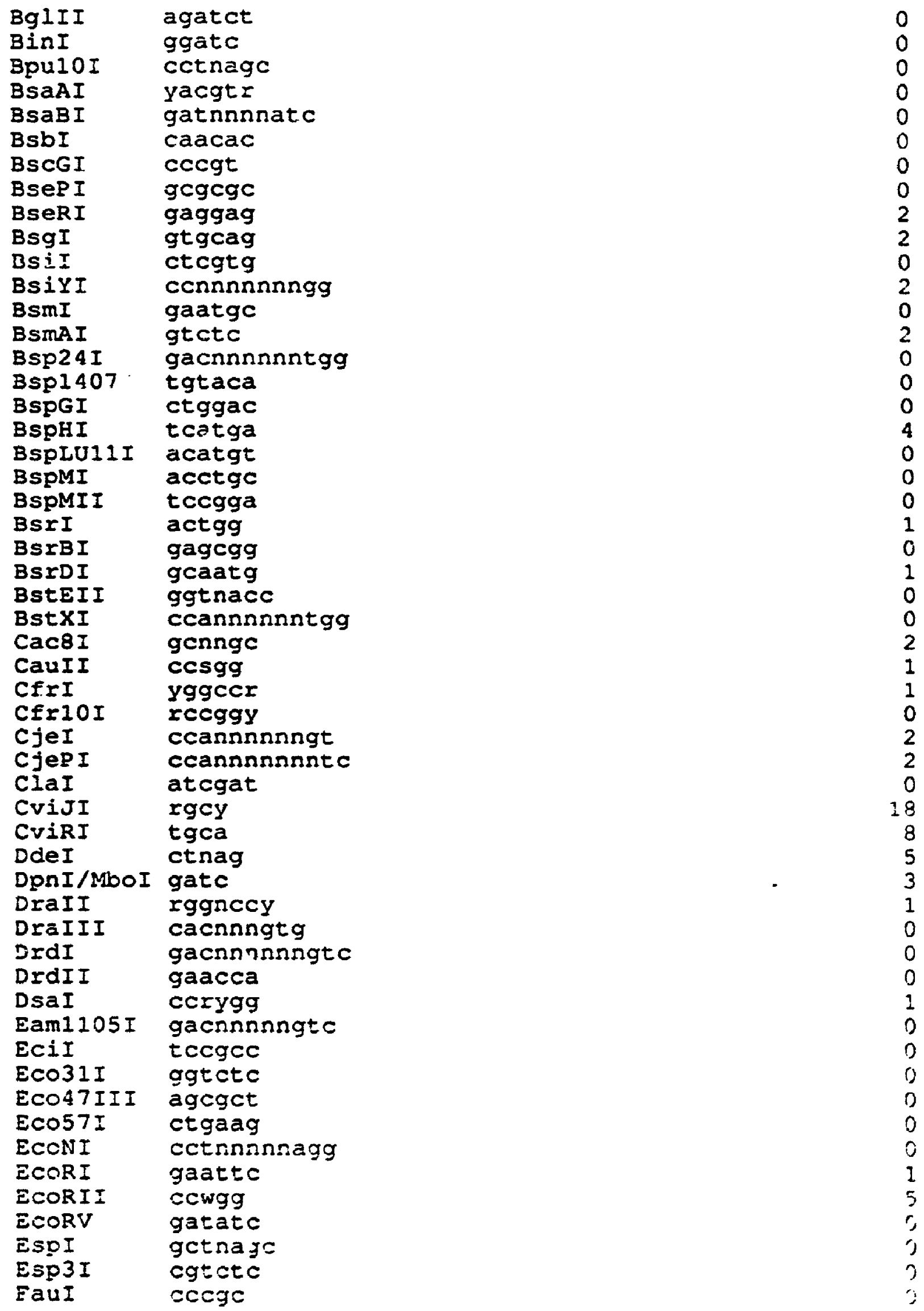




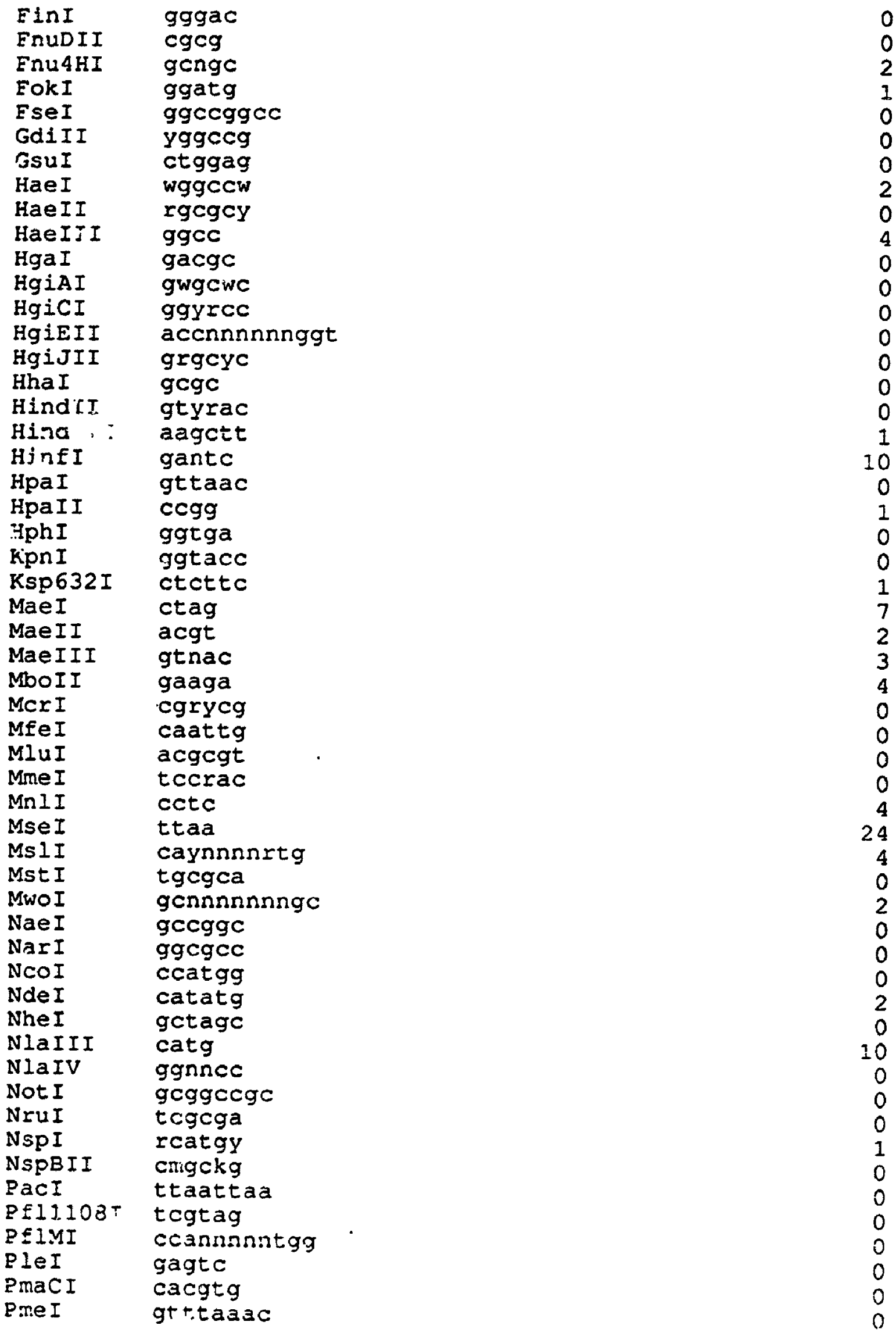




\begin{tabular}{|c|c|c|}
\hline PpuMI & rggwccy & 0 \\
\hline PshAI & gacnnnngtc & 0 \\
\hline BstI & ctgcag & 1 \\
\hline PvuI & cgatcg & 0 \\
\hline PvuII & cagctg & 0 \\
\hline R.LeAI & cccaca & 1 \\
\hline RsaI & gtac & 3 \\
\hline RSIII & cggwceg & 0 \\
\hline SacI & gagctc & 0 \\
\hline SacII & $\operatorname{ccg} \operatorname{cgg}$ & 0 \\
\hline SalI & gtcgac & 0 \\
\hline SapI & getctec & 0 \\
\hline SauI & Cr-ullayg & 1 \\
\hline ScaI & agtact & 1 \\
\hline ScrEI & cengg & 6 \\
\hline SduI & gdgche & 0 \\
\hline SecI & conngg & 2 \\
\hline SexAI & accwggt & 1 \\
\hline SEaNI & gcatc & 2 \\
\hline SfeI & ctryag & 1 \\
\hline SfiI & ggccnnnnnggcc & 0 \\
\hline SgfI & gcgatcgc & 0 \\
\hline SgrAI & crccggyg & 0 \\
\hline SmaI & $\operatorname{cccggg}$ & 0 \\
\hline SnaI & gtatac & 0 \\
\hline SnaBI & tacgta & 0 \\
\hline SpeI & $\operatorname{act} 25 t$ & 1 \\
\hline SphI & gcatgc & 0 \\
\hline Spli & cgtacg & 0 \\
\hline SrfI & gccogggc & 0 \\
\hline Sse8387I & cctgcagg & 0 \\
\hline SspI & aatatt & 1 \\
\hline StuI & aggcet & 0 \\
\hline Sty I & ccwwgg & 0 \\
\hline SwaI & atttaeat & 2 \\
\hline TaqI & tcga & 0 \\
\hline TaqIIa & caccca & 0 \\
\hline TaqIIb & gaccga & 0 \\
\hline TEiI & gawtc & 6 \\
\hline TseI & gcwgc & 2 \\
\hline Tsp4 5 I & gtsac & 0 \\
\hline TSpEI & aatt & 21 \\
\hline TthIIII & gacnnngtc & 0 \\
\hline Tth111II & caarca & 0 \\
\hline VspI & attaat & 3 \\
\hline XbaI & tctaga & 0 \\
\hline$x \mathrm{cmI}$ & ccannnnnnnnntgg & 1 \\
\hline XhoI & ctcgag & 0 \\
\hline XhoII & rgatcy & 1 \\
\hline Xma I II & $\operatorname{cggccg}$ & 0 \\
\hline $\mathrm{XmnI}$ & gaannnntt: & 2 \\
\hline Tota 1 & & 23 \\
\hline
\end{tabular}




\section{Appendix $\mathbf{J}$}

\section{Human Interferon-Alpha 8 Gene}

\section{J.1 Nucleotide Sequence}

The genomic DNA sequence of the human interferon-alpha 8 gene was retrieved from the Genbank sequence file hsifnas (Henco e.. al., 1985) (Benson et. al., 1993).

1 aggggtcatc catctgaacc agctcagcag catccacaac atctacaatg gecttgactt 61 tttattact ggtggeccta gtggtgctca getacaagtc attcagctct ctgggctgtg 121 atctgectca gactcacage ctgggtaaca ggagggectt gatactcctg geacaaatgc 181 gaagaatctc tccttctce tgectgaagg acagacatga ctttgaattc ccccaggagg 241 agtttgatga taaacagttc cagaaggetc aagecatctc tgtcctccat gagatgatcc 301 agcagacctt caacctzttc agcacaaagg actcatctgc tgcttggat gagacccttc 361 tagatgaatt ctacatcgaa cttgaccagc agctgaatga cctggagtce tgtgtgatgc 421 aggaagtggg ggtgatagag tctcccctga tgtacgagga ctccatcctg getgtgagga 481 aatacttcca aagaatcact ctatatctga cagagaagaa atacagctct tgtgcctggg 541 aggttgtcag agcagaaatc atgagatcct tctcttatc aatcaacttg caaaaaagat 601 tgaagagtaa ggaatgagac ctggtacaac acg 


\section{J.2 Restriction Endonuclease Sites}

The human interferon-alpha 8 gene restriction endonuclease recognition sites are arranged by ascending sequence location.

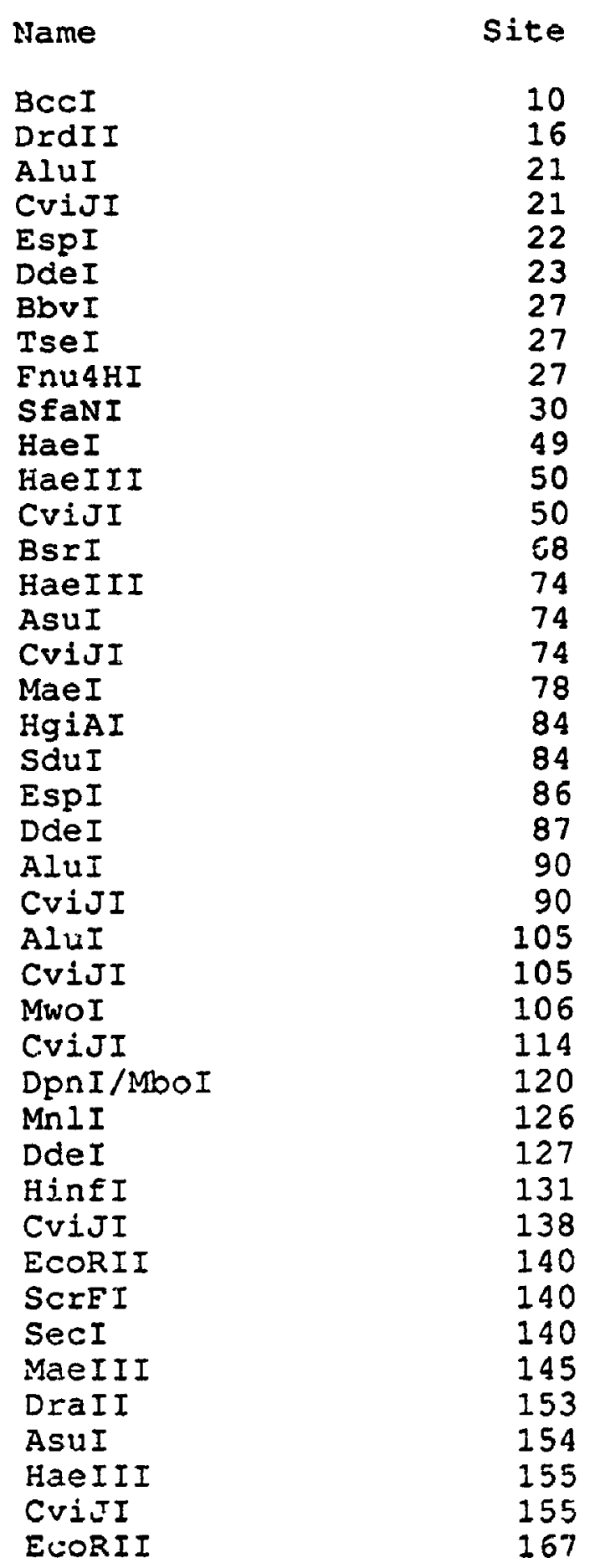




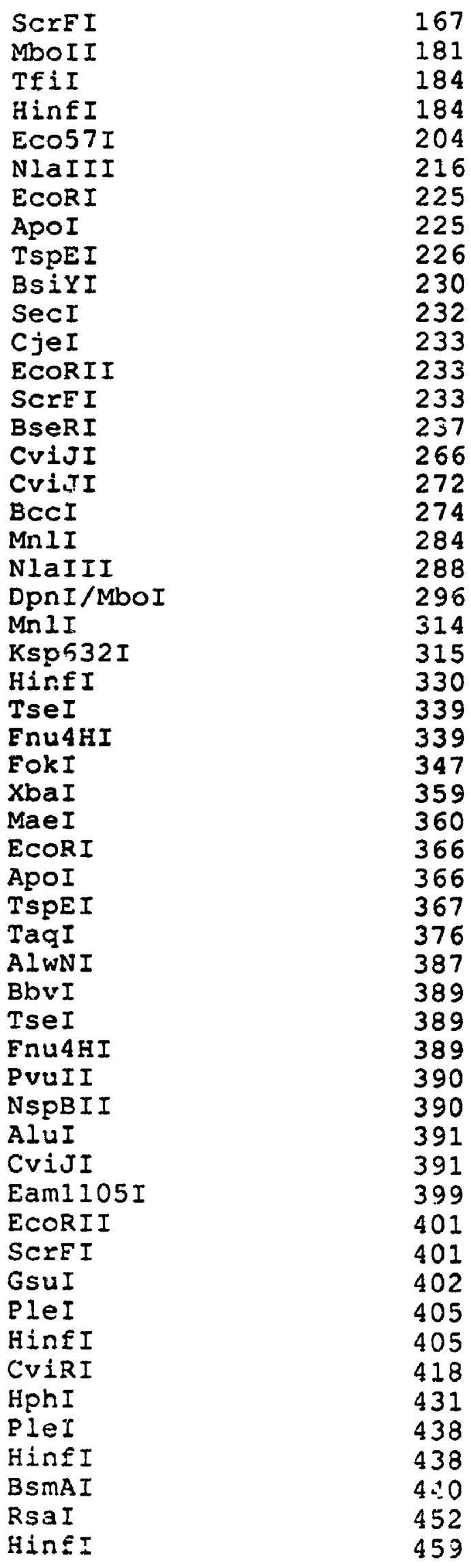




$\begin{array}{ll}\text { BCcI } & 463 \\ \text { EcoRII } & 467 \\ \text { ScrEI } & 467 \\ \text { CVIJI } & 470 \\ \text { XmnI } & 479 \\ \text { TfiI } & 493 \\ \text { HinfI } & 493 \\ \text { MboII } & 515 \\ \text { AluI } & 525 \\ \text { CviJI } & 525 \\ \text { EcoRII } & 535 \\ \text { ScrFI } & 535 \\ \text { SecI } & 535 \\ \text { BspHI } & 559 \\ \text { NlaIII } & 560 \\ \text { XhoII } & 564 \\ \text { DpnI/MboI } & 565 \\ \text { CViRI } & 589 \\ \text { MboII } & 602 \\ \text { SexAI } & 619 \\ \text { EcoRII } & 620 \\ \text { ScrEI } & 620 \\ \text { RsaI } & 624 \\ \text { BsbI } & 627\end{array}$

The human interferon-alpha 8 gene restriction endonuclease recognition sites are arranged by enzyme.

Name

AluI

AluI

AluI

AluI

AluI

AlWNI

ApoI

ApoI

AsuI

AsuI

BbvI

BbvI

BCCI

BCCI

BCCI

BsbI

BseRI

BSIYI

BJmAI

BSpH:

Site
21
90
105
391
525
387
225
366
74
154
27
389
10
274
463
627
237
230
440
559




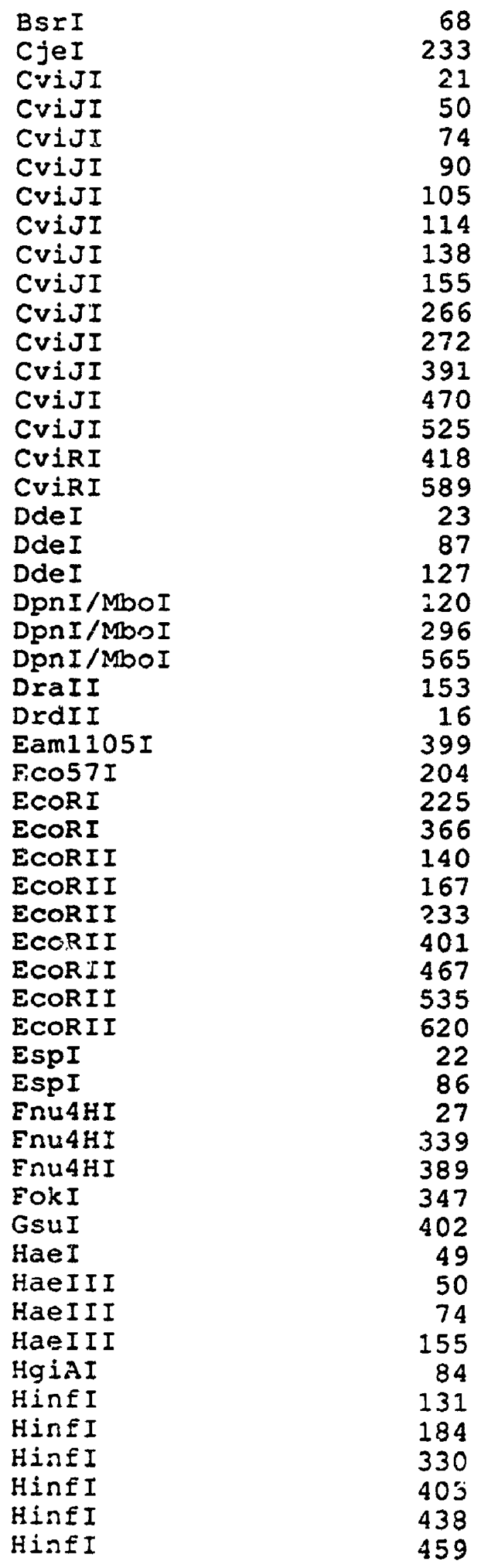




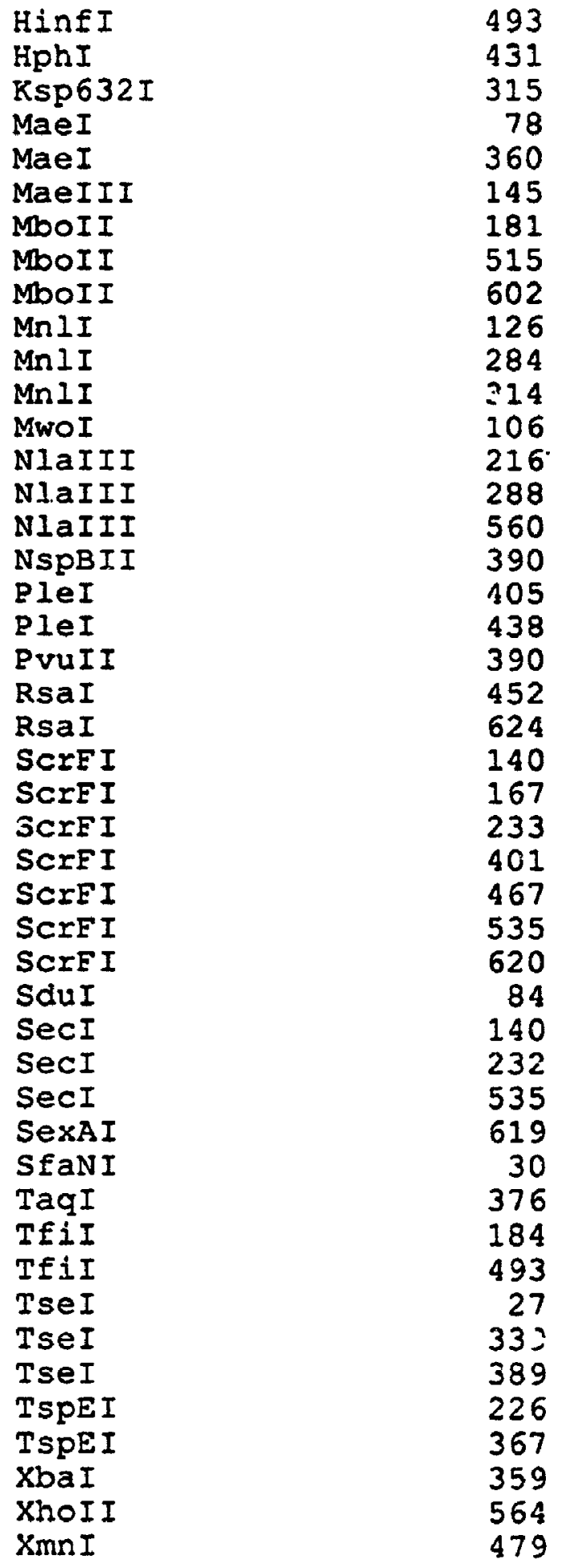


The human interferon-alpha 8 gene restriction endonuclease recognition sites are summarized by enzyme.

\begin{tabular}{|c|c|c|}
\hline Name & Pattern & Sites \\
\hline AatII & gacgtc & 0 \\
\hline$A C C I$ & gtmkac & 0 \\
\hline ACII & $\operatorname{cog} r$ & 0 \\
\hline ACII & aacgtt & 0 \\
\hline ACYI & gregyc & 0 \\
\hline AfIII & cttaag & 0 \\
\hline AfIIII & acrygt & 0 \\
\hline AgeI & accogyt & 0 \\
\hline AhaIII & tttaaa & 0 \\
\hline AIUI & agc: & 5 \\
\hline AIWNI & cägnnncig & \\
\hline Apa I & gggecc & 0 \\
\hline ApaBI & gcannnnntgc & 0 \\
\hline ApaII & gigcac & 0 \\
\hline ApoI & raatty & 2 \\
\hline AsreI & ggcgcgcc & \\
\hline AsuI & ggnce & 2 \\
\hline AsuII & ttcgaa & \\
\hline AvaI & cycgrg & 0 \\
\hline AvaII & ggwec & \\
\hline AvaIII & atgcat & 0 \\
\hline AvrII & cctagg & \\
\hline BaeI & acnnnngtayc & 0 \\
\hline $\mathrm{BaII}$ & tggcca & 0 \\
\hline BamH I & ggatcc & \\
\hline BbvI & geagc & sina \\
\hline BbvII & gaagac & \\
\hline$B C C I$ & ccatc & \\
\hline Bce83I & cttgag & \\
\hline BcefI & $\operatorname{acggc}$ & \\
\hline$B C g I$ & gcannnnnntcg & \\
\hline BcíI & tgatca & \\
\hline Bet I & wccggw & \\
\hline $\mathrm{Bg} I \mathrm{I}$ & gccnnnnnggc & \\
\hline$B g I^{\top} I$ & agatct & \\
\hline BinI & gqatc & \\
\hline Bpu10I & cetnage & \\
\hline BSaAI & yacgtr & \\
\hline BsaBI & gatñnnatc & \\
\hline BsbI & caacac & \\
\hline BscGI & cccgt & \\
\hline Bse? I & $\operatorname{gcgcgc}$ & \\
\hline BseRI & gaggag & \\
\hline BSGI & $g t g c a g$ & \\
\hline BSiI & ctcgtg & \\
\hline BSIYI & connnnnnngg & \\
\hline BsmI & gaatgc & \\
\hline
\end{tabular}




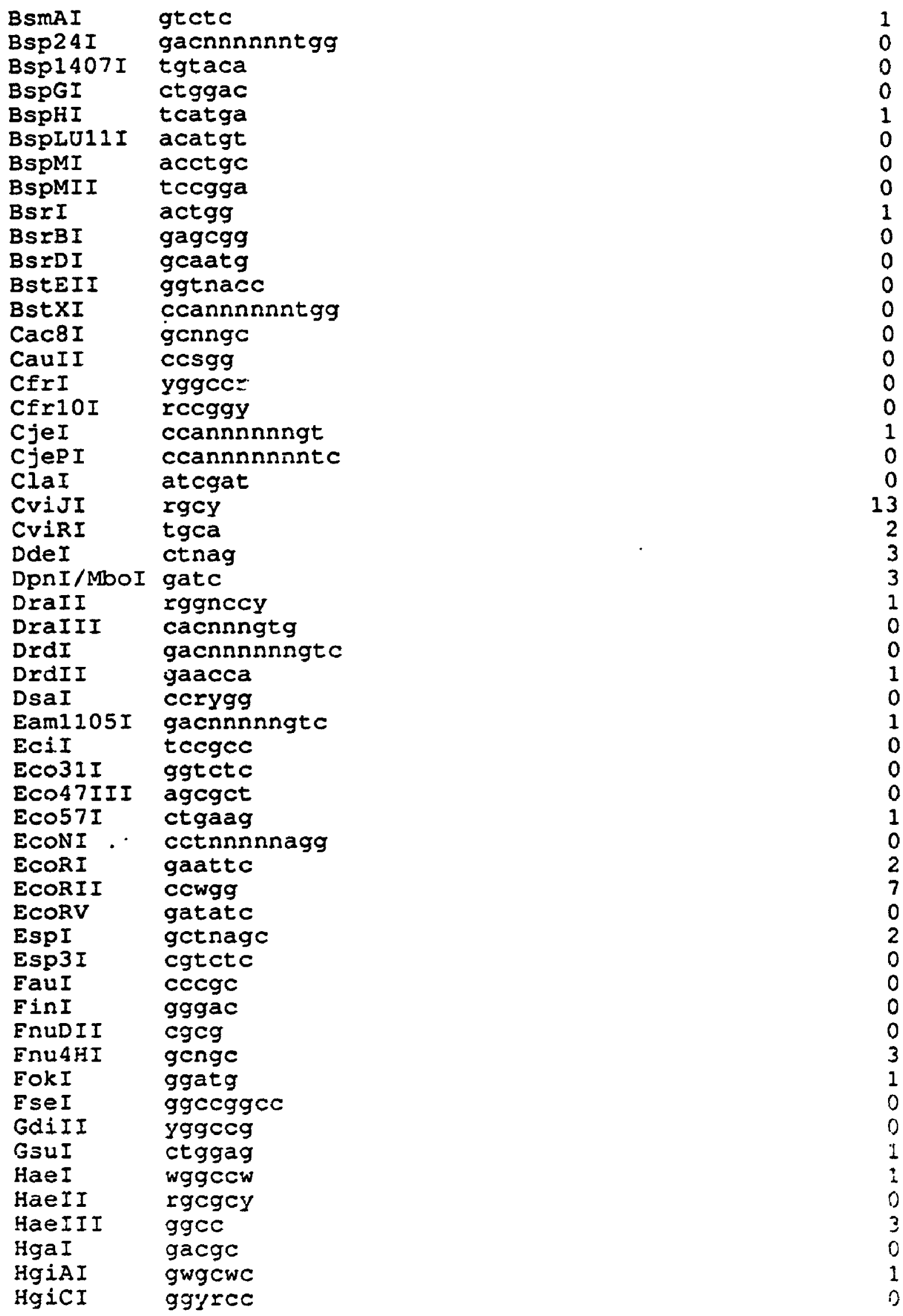




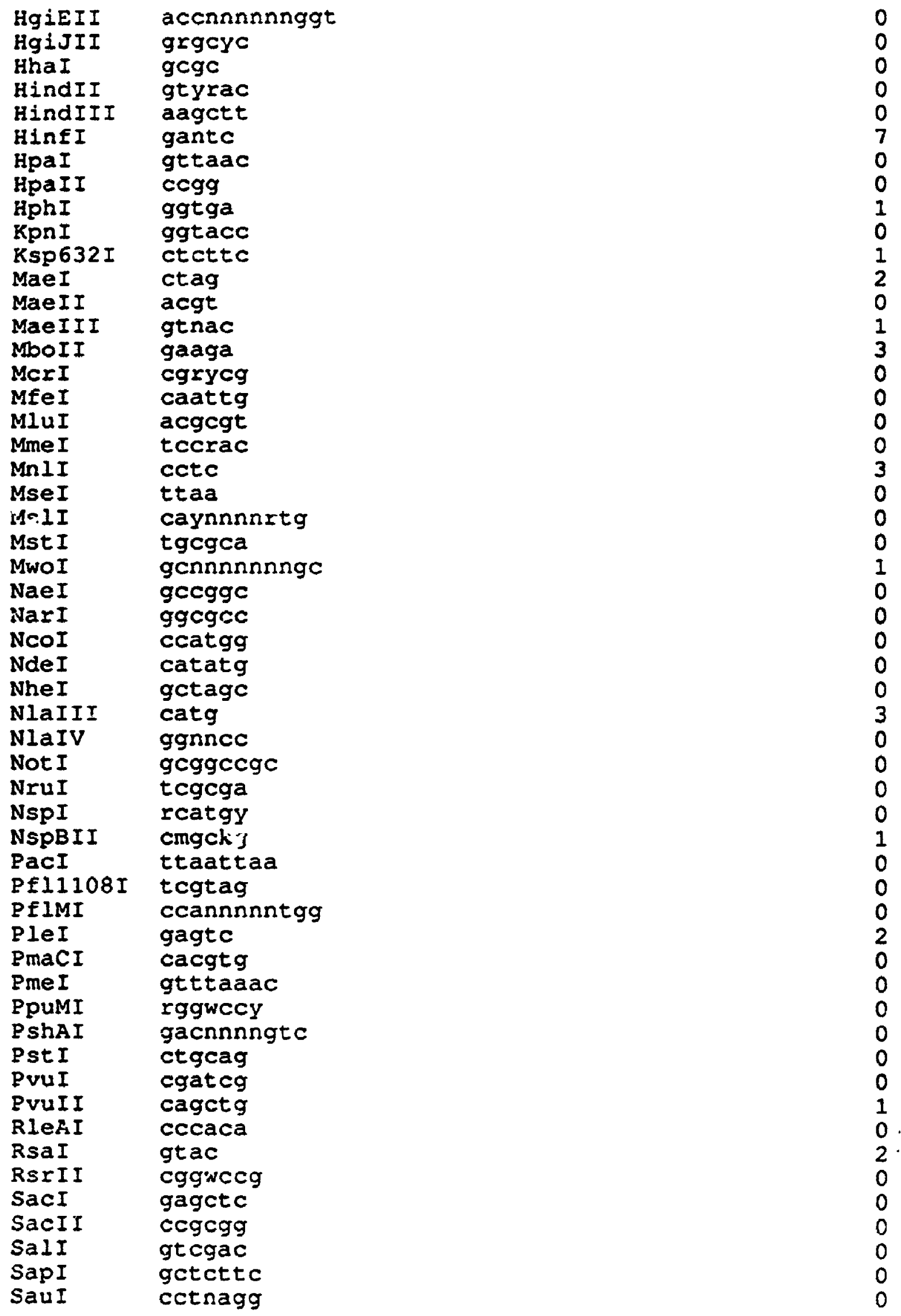




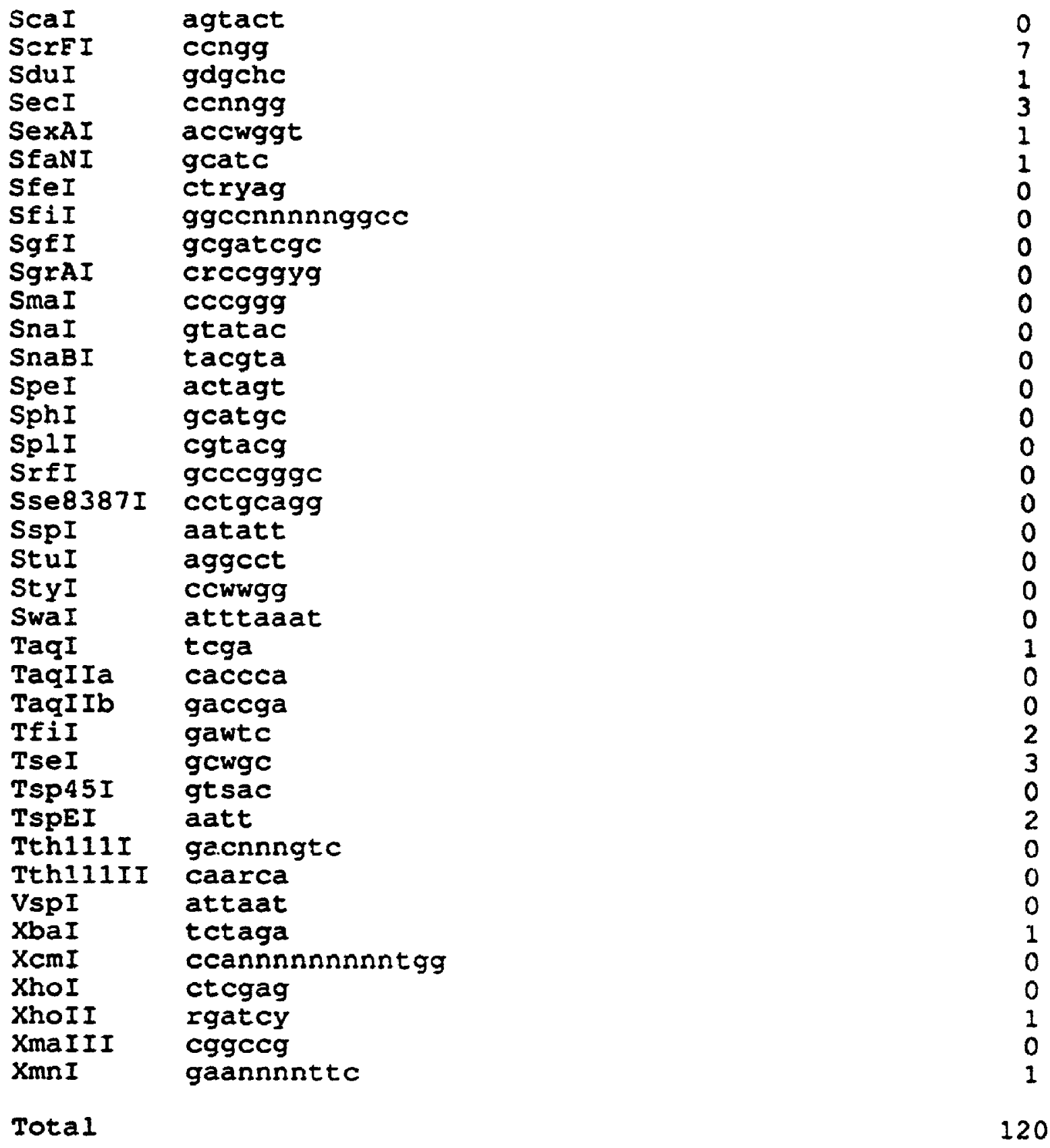




\section{Appendix $K$}

\section{Human Interferon-Alpha 13 Gene}

\section{K.1 Nucleotide Sequence}

The genomic DNA sequence of the human interferon-alpha 13 gene was retrieved

from the Genbank sequence file hsifrl8 (Mantei et. al., 1980) (Todokoro et. al., 1984)

(Benson et. al., 1993).

1 aaacaaaatg gttgagaaac acggctctaa actcatgtaa agagttcaag aaggaaagca

61 aaaacagaaa tggaaagtgg tccagaagca ttaagaaagt ggaaatcagt atgttcccta

121 ttaaggcat ctgcaggaag caaagectic agagaaceta gagcccaagg ttcagagtca

181 cccatctcag caagcccaga agcatctgca atatctatga iggcctcgec ctttgettra

241 ctgatggtec tggtggtgct cagctgcaag tcaagctgct ctctgggctg tgatctecet

301 gettacccaca gectggataa caggaggace ttgatgctec tggcacaaat gageagaatc

361 t::ectect cctgtctgat ggacagacat gacttggat ttccccagga ggaztttgat

421 ggeaaccagt tccagaagge tccagecatc tctgtectcc atgagctgat ccagcagatc

481 ttcaacctct ttaccacaaa agattcatct getgcttggg atgaggacet cctagycaaa

541 ttctgcaccg aactctacca geagetgaat gacttggaag cctgtgtgat geaggaggag

601 agggtgggag aaactccect gatgaatgeg gactccatct tggetgtgaa gaaatacttc

661 cgaagaatca ctctctatct gacagagaag aaatacagce cttgtgecte Egaggttgtc

721 agagcagaaa tcatgagatc cctctctta tcaacaaact tgcaagaaag attaaggagg

781 aaggaataac acctggtcca acatgaaaac auttcttatt gactcatata ccaggtcacg

841 ctttcatgaa ttetgtcatt tcaaagactc tcacecctge tataactatg accatgctga

901 taaactgatt tatctattta aatattatt tagctattca taagatttaa attattttg

961 ttcatataac atcatgtgca tettacact gtggttagtg taataaaaca tgttcettat

1021 . tctactcaa ttcattatt tatgttgttc attaaactt tactatagga acttcetgta

1081 tgtgttcatt cttaataca taaattce 


\section{K.2 Restriction Endonuclease Sites}

The human interferon-alpha 13 gene restriction endonuclease recognition sites are arranged by ascending sequence location.

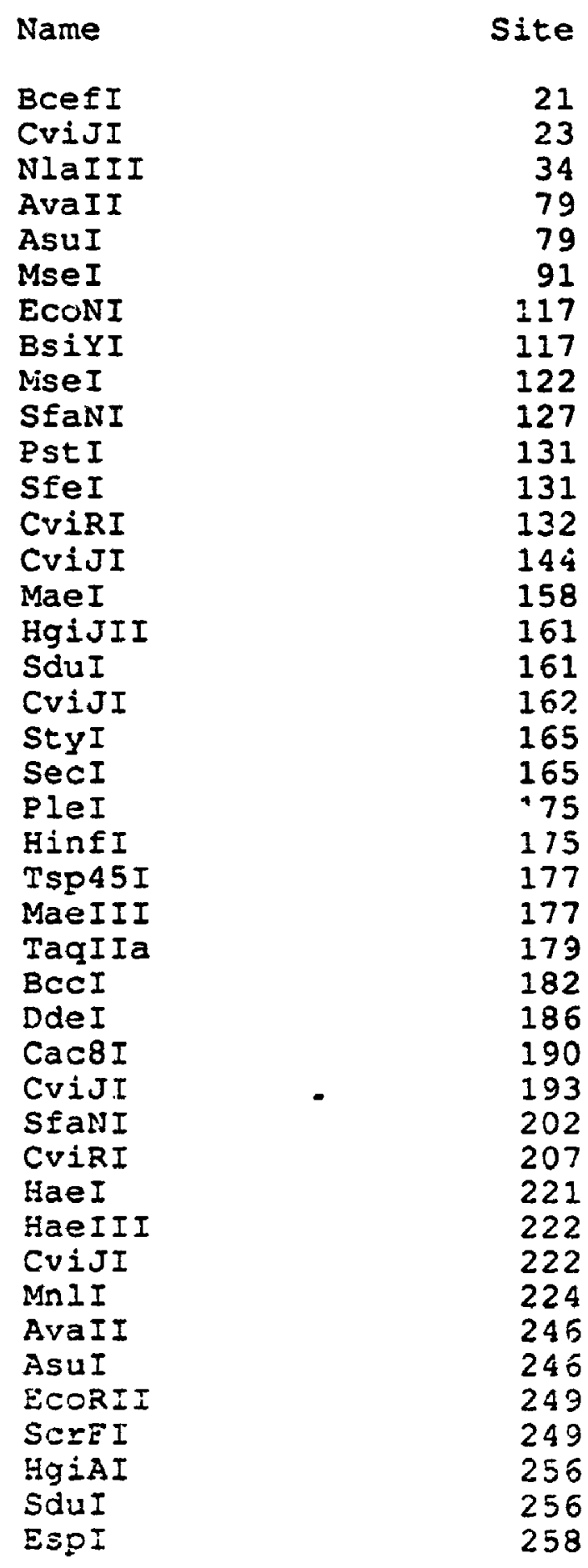



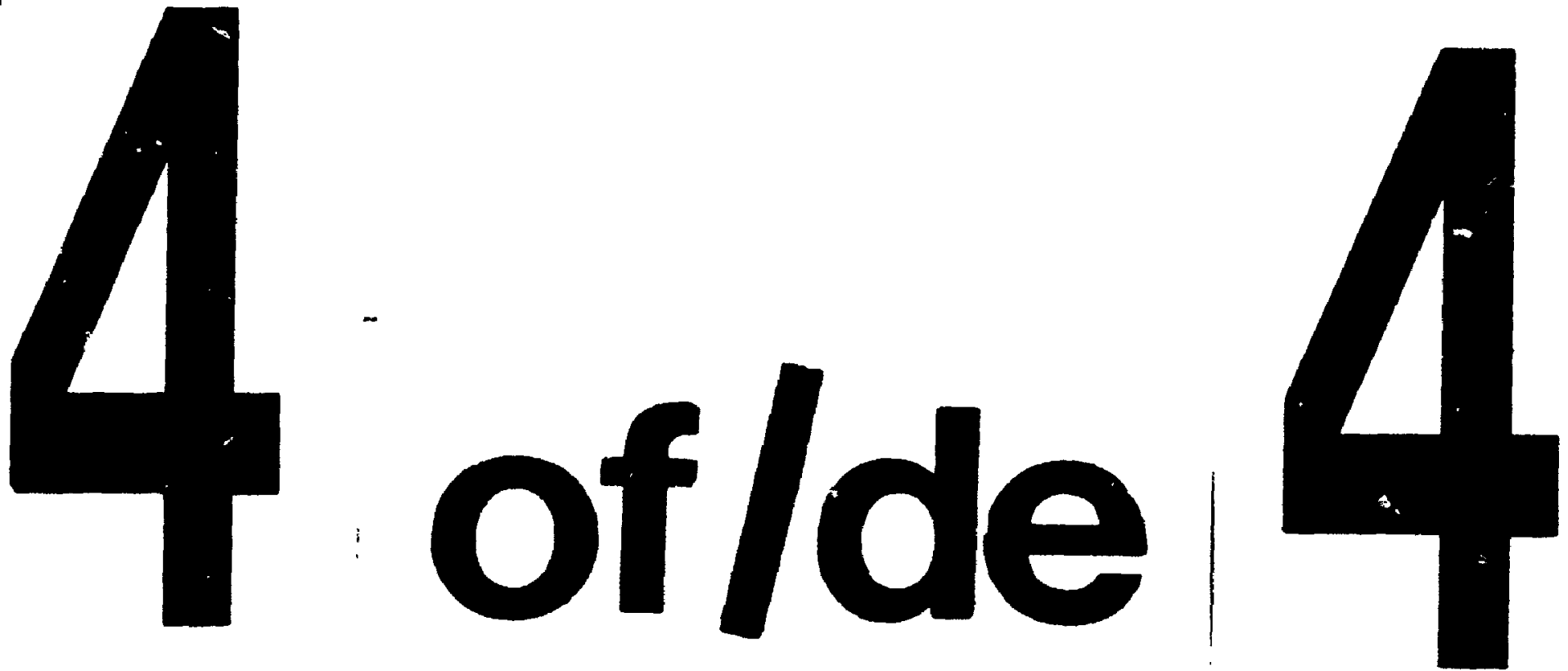

PM-1 3\%"x4" PHOTOGRAPHIC MICROCOPY TARCET NBS 1010a ANSI/ISO *2 EQUIVALENT

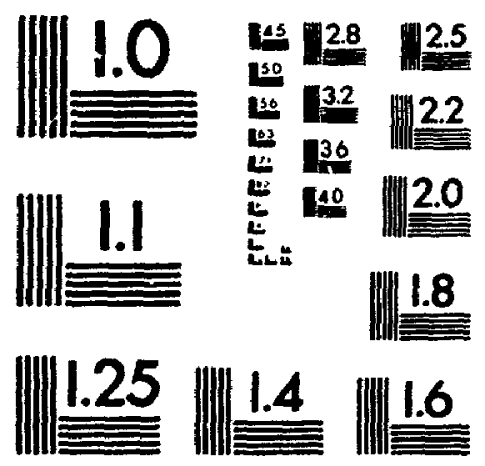

PRECISIONSM RESOLUTION TARGETS 


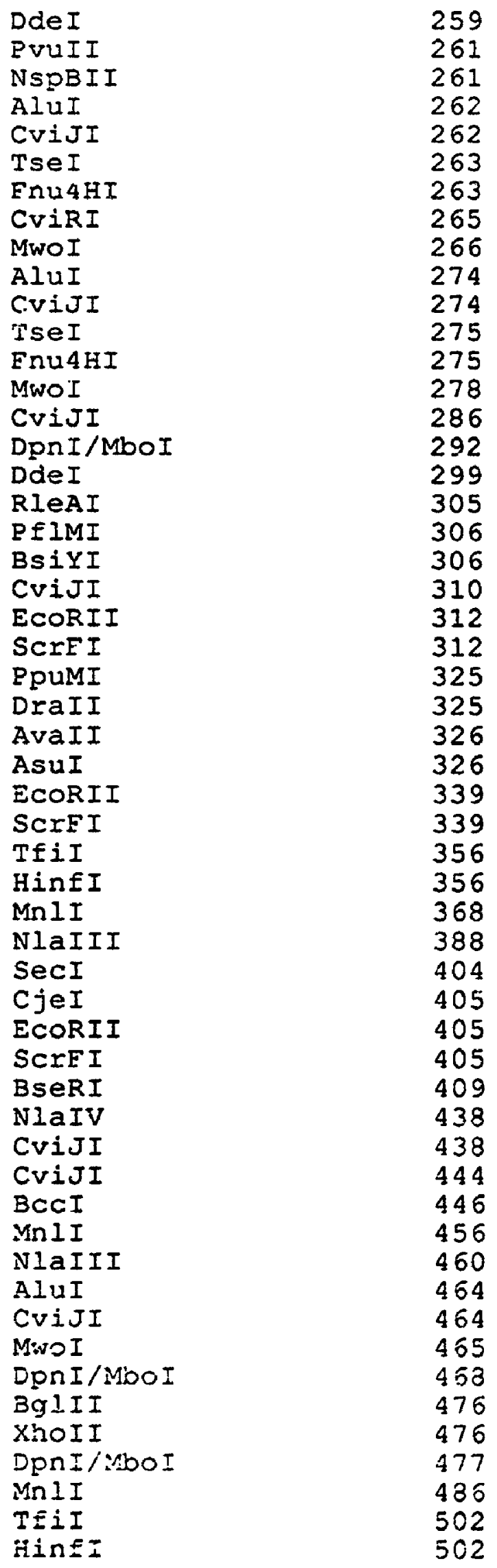




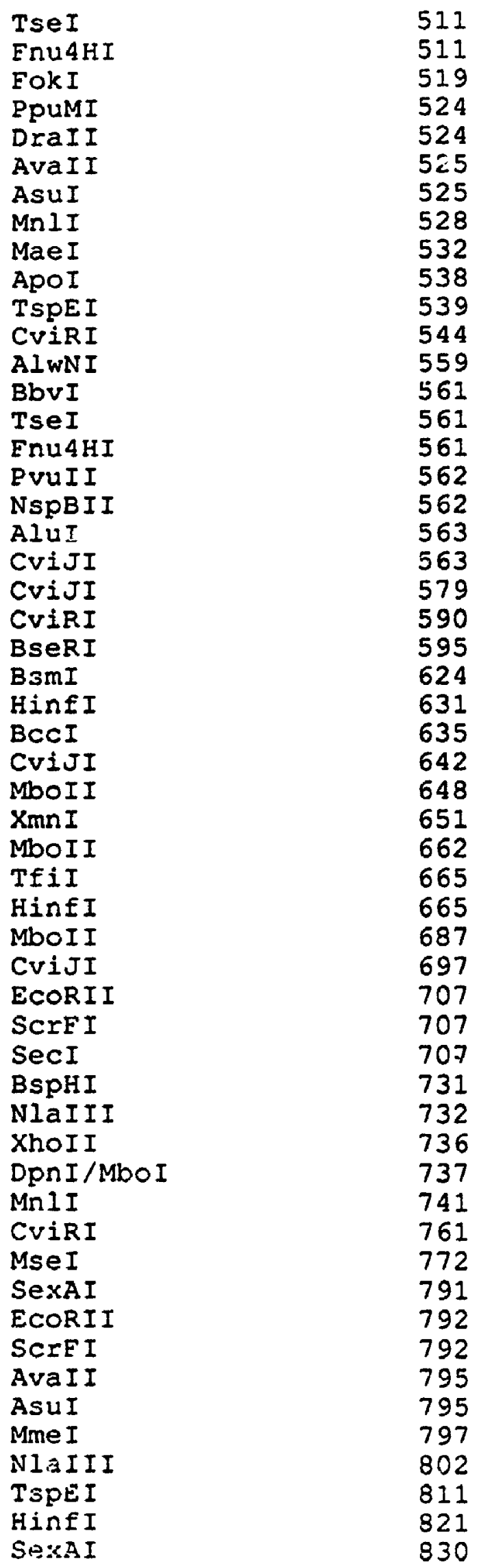




$\begin{array}{lr}\text { EcoRII } & 831 \\ \text { ScrEI } & 831 \\ \text { Tsp45I } & 835 \\ \text { MaeIII } & 835 \\ \text { BspHI } & 844 \\ \text { NlaIII } & 845 \\ \text { EcoRI } & 848 \\ \text { ApoI } & 848 \\ \text { TspEI } & 849 \\ \text { HinfI } & 866 \\ \text { NlaIII } & 893 \\ \text { SwaI } & 916 \\ \text { AhaIII } & 917 \\ \text { MseI } & 918 \\ \text { SspI } & 921 \\ \text { AluI } & 932 \\ \text { CviJI } & 932 \\ \text { SwaI } & 945 \\ \text { AhaIII } & 946 \\ \text { MseI } & 947 \\ \text { TspEI } & 950 \\ \text { NlaIII } & 973 \\ \text { CviRI } & 177 \\ \text { SfaNI } & 978 \\ \text { BspIUIII } & 1008 \\ \text { AfIIII } & 1008 \\ \text { NspI } & 1008 \\ \text { NlaIII } & 1009 \\ \text { TspEI } & 1029 \\ \text { MseI } & 1052 \\ \text { SEeI } & 1063 \\ \text { MseI } & 1093 \\ \text { ApoI } & 1102 \\ \text { TspEI } & 1103\end{array}$

The human interferon-alpha 13 gene restriction endonuclease recognition sites are arranged by enzyme.

Name

AfIIII

AhaIII

AhaIII

AluI

AluI

AluI

A.UI

AluI

Alivi

ApoI
Site

1008

917

946

262

274

464

563

932

559

538 


\begin{tabular}{|c|c|}
\hline 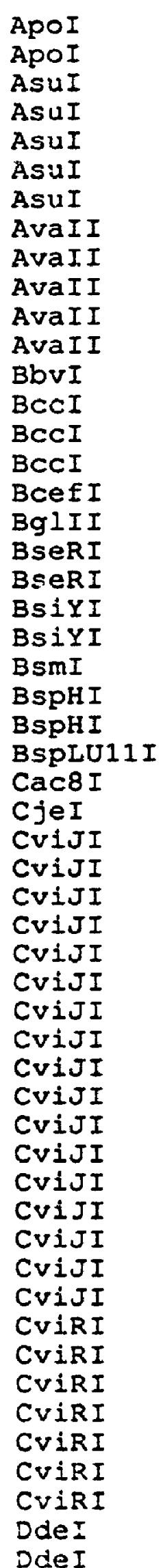 & $\begin{array}{r}848 \\
1102 \\
79 \\
246 \\
326 \\
525 \\
795 \\
79 \\
246 \\
326 \\
525 \\
795 \\
561 \\
182 \\
446 \\
635 \\
21 \\
476 \\
409 \\
595 \\
117 \\
306 \\
624 \\
731 \\
844 \\
1008 \\
190 \\
405 \\
23 \\
144 \\
162 \\
193 \\
222 \\
262 \\
274 \\
286 \\
310 \\
438 \\
444 \\
464 \\
563 \\
579 \\
642 \\
697 \\
932\end{array}$ \\
\hline
\end{tabular}




$\begin{array}{lr}\text { DdeI } & 299 \\ \text { DpnI/MboI } & 292 \\ \text { DpnI/MboI } & 468 \\ \text { DpnI/MboI } & 477 \\ \text { DpnI/MboI } & 737 \\ \text { DraII } & 325 \\ \text { DraII } & 524 \\ \text { EcoNI } & 117 \\ \text { EcoRI } & 848 \\ \text { EcoRII } & 249 \\ \text { EcoRII } & 312 \\ \text { EcoRII } & 339 \\ \text { EcoRII } & 405 \\ \text { EcoRII } & 707 \\ \text { EcoRII } & 792 \\ \text { EccRII } & 831 \\ \text { EsiI } & 258 \\ \text { Eiu4HI } & 263 \\ \text { Enu4HI } & 275 \\ \text { Fnu4HI } & 511 \\ \text { EnutHI } & 561 \\ \text { EokI } & 519 \\ \text { HaeI } & 221 \\ \text { HaeIII } & 222 \\ \text { HgiAI } & 256 \\ \text { HgiJII } & 1051 \\ \text { HinfI } & 1093 \\ \text { HinfI } & \end{array}$




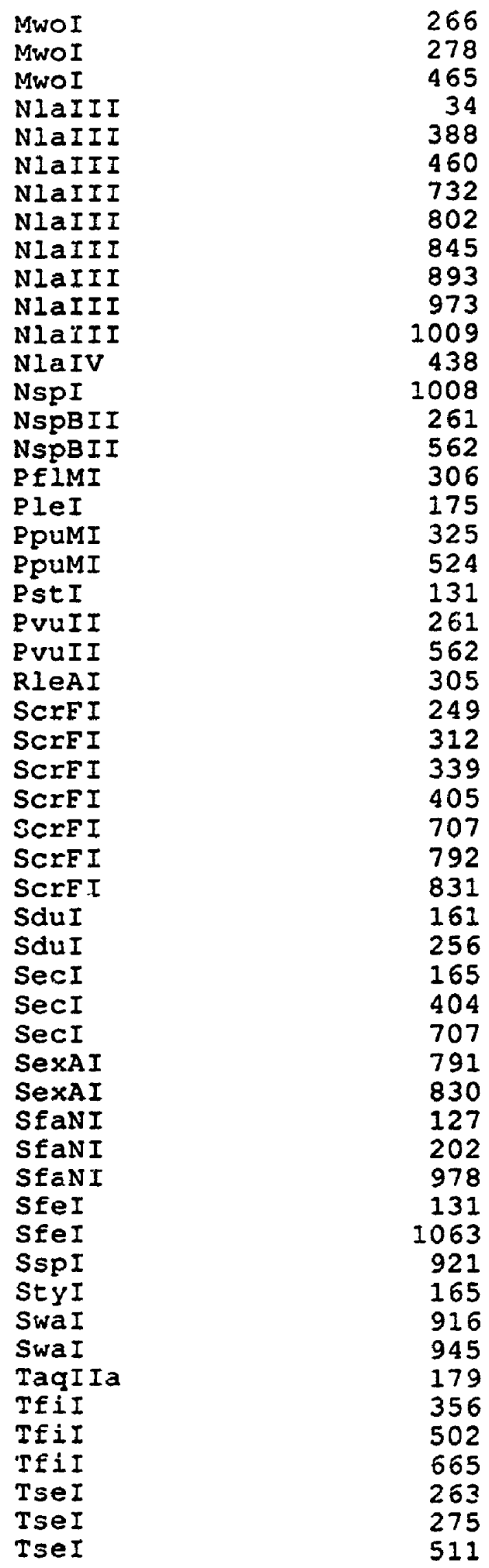


TseI

Tsp4 5 I

Tsp4 5 I

TspEI

TSpEI

TSPEI

TSPEI

TSPEI

TspEI

XhoII

XhoII

XmnI
561

177

835

539

811

849

950

1029

1103

476

736

651

The human interferon-alpha 13 gene restriction endonuclease recognition sites are summarized by enzyme.

\begin{tabular}{|c|c|c|}
\hline Name & Pattern & Sites \\
\hline AatII & gacgtc & 0 \\
\hline AccI & gtmkac & 0 \\
\hline AciI & $\operatorname{ccgc}$ & 0 \\
\hline ACII & aacgtt & 0 \\
\hline AcyI & grcgyc & 0 \\
\hline AfIII & cttaag & 0 \\
\hline AfIIII & acrygt & 1 \\
\hline AgeI & accggt & $\overrightarrow{0}$ \\
\hline AhaIII & tttaaa & 2 \\
\hline AluI & agct & 5 \\
\hline AlWNI & cagnn jetg & 1 \\
\hline ApaI & gggce. & 0 \\
\hline ApaBI & gcannnnntgc & 0 \\
\hline ApaLI & gtgcac & 0 \\
\hline ApoI & raatty & 3 \\
\hline AscI & ggcgcgcc & 0 \\
\hline AsuI & ggnce & 5 \\
\hline AsuII & tecgaa & 0 \\
\hline AvaI & cycgrg & 0 \\
\hline AvaII & SGNCC & 5 \\
\hline AvaIII & atgcat & 0 \\
\hline AvrII & cctagg & 0 \\
\hline BaeI & acnnnngtayc & 0 \\
\hline BaiI & tggcca & 0 \\
\hline BamHI & ggatcc & $\mathrm{c}$ \\
\hline BbvI & gcagc & 1 \\
\hline BbvII & gaagac & 0 \\
\hline$B C C I$ & ccatc & 3 \\
\hline Bce83I & cttgag & 0 \\
\hline BcefI & acggc & $i$ \\
\hline$B C g I$ & gcannnsnntcq & 0 \\
\hline BCII & tgatca & \\
\hline
\end{tabular}




\begin{tabular}{|c|c|}
\hline $\begin{array}{l}\text { BetI } \\
\text { BgII } \\
\text { BgIII } \\
\text { BinI } \\
\text { BpuIOI } \\
\text { BsaAI } \\
\text { BsaBI } \\
\text { BsbI } \\
\text { BscGI } \\
\text { BsePI } \\
\text { BseRI } \\
\text { BsgI } \\
\text { BsiI } \\
\text { BsiYI } \\
\text { BsmI } \\
\text { BsmAI } \\
\text { Bsp24I } \\
\text { BspI407I } \\
\text { BspGI } \\
\text { BspHI } \\
\text { BspIUIII } \\
\text { BspMI } \\
\text { BspMII } \\
\text { BsrI } \\
\text { BsrBI } \\
\text { BsIDI } \\
\text { BstEII } \\
\text { BstXI } \\
\text { Cac8I } \\
\text { CauII } \\
\text { CfrI } \\
\text { CExI0I } \\
\text { CjeI } \\
\text { CjePI } \\
\text { CIaI } \\
\text { CviJI } \\
\text { CviRI } \\
\text { DdeI } \\
\text { DpnI/MboI } \\
\text { DiaII } \\
\text { DraIII } \\
\text { DrdI } \\
\text { DrdII } \\
\text { DsaI } \\
\text { EamII05I } \\
\text { EciI } \\
\text { Eco3II } \\
\text { Eco47III } \\
\text { Eco57I } \\
\text { EcoNI } \\
\text { EcoRI } \\
\text { EcoRII } \\
\text { EcoRV } \\
\text { EspI }\end{array}$ & 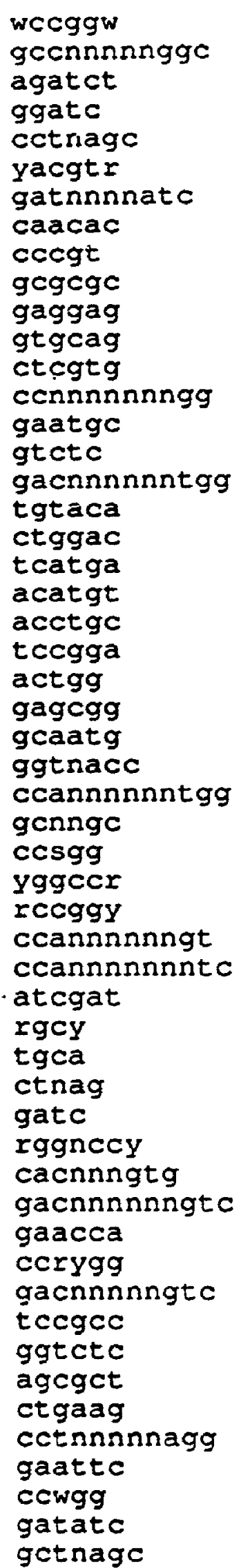 \\
\hline
\end{tabular}




\begin{tabular}{|c|c|c|}
\hline $\operatorname{Esp} 3 I$ & cgtctc & 0 \\
\hline FauI & $\operatorname{cccgc}$ & 0 \\
\hline EinI & gggac & 0 \\
\hline EnUDII & $\operatorname{cgcg}$ & 0 \\
\hline EnU4HI & gcngc & 4 \\
\hline EokI & ggatg & 1 \\
\hline EseI & ggccggcc & 0 \\
\hline GdiII & yggccg & 0 \\
\hline Gsur & ctggag & 0 \\
\hline HaeI & wggccw & 1 \\
\hline HaeII & rgcgcy & 0 \\
\hline HaeIII & ggce & 1 \\
\hline Hga I & gacgc & 0 \\
\hline HgiAI & gwgcwC & 1 \\
\hline HgiCI & ggyrce & $\mathrm{C}$ \\
\hline HgiEII & accnnnnnnggt & 0 \\
\hline HgiJII & grgcyc & 1 \\
\hline HhaI & gcgc & 0 \\
\hline HindII & gtyrac & 0 \\
\hline HindIII & aagctt & 0 \\
\hline HinfI & yantc & 7 \\
\hline HраI & gttaac & 0 \\
\hline HpaII & cogg & 0 \\
\hline HphI & ggtga & 0 \\
\hline KpnI & ggtacc & 0 \\
\hline Ksp632I & ctcttc & 0 \\
\hline MaeI & ctag & 2 \\
\hline MaeII & $\operatorname{acgt}$ & 0 \\
\hline MaeIII & gtrac & 2 \\
\hline MboII & gaaga & 3 \\
\hline McrI & cgryeg & 0 \\
\hline MfeI & caattg & 0 \\
\hline MluI & $\operatorname{acgcg} t$ & 0 \\
\hline Mime I & tccrac & 1 \\
\hline MnlI & $\cot c$ & 6 \\
\hline MseI & ttaa & 7 \\
\hline Ms1I & caynnnnxtg & 0 \\
\hline Mst I & tgcgca & 0 \\
\hline MwoI & gcnnnnnnngc & 3 \\
\hline NaeI & gccgge & 0 \\
\hline NarI & ggcgce & 0 \\
\hline NCOI & ccatgg & 0 \\
\hline NdeI & catatg & 0 \\
\hline NheI & gctagc & 0 \\
\hline NlaIII & catg & 9 \\
\hline NIaIV & ggnnes & 1 \\
\hline Not I & gcggccgc & 0 \\
\hline NruI & tcgcga & 0 \\
\hline NspI & rcatgy & 1 \\
\hline NspBII & cmgckg & 2 \\
\hline PacI & ttaattaa & 0 \\
\hline$P f 11108 I$ & tcgtag & 0 \\
\hline $\begin{array}{l}\text { PfIMI } \\
\text { PleI }\end{array}$ & $\begin{array}{l}\text { ccannnnntgg } \\
\text { qaqtc }\end{array}$ & \\
\hline
\end{tabular}




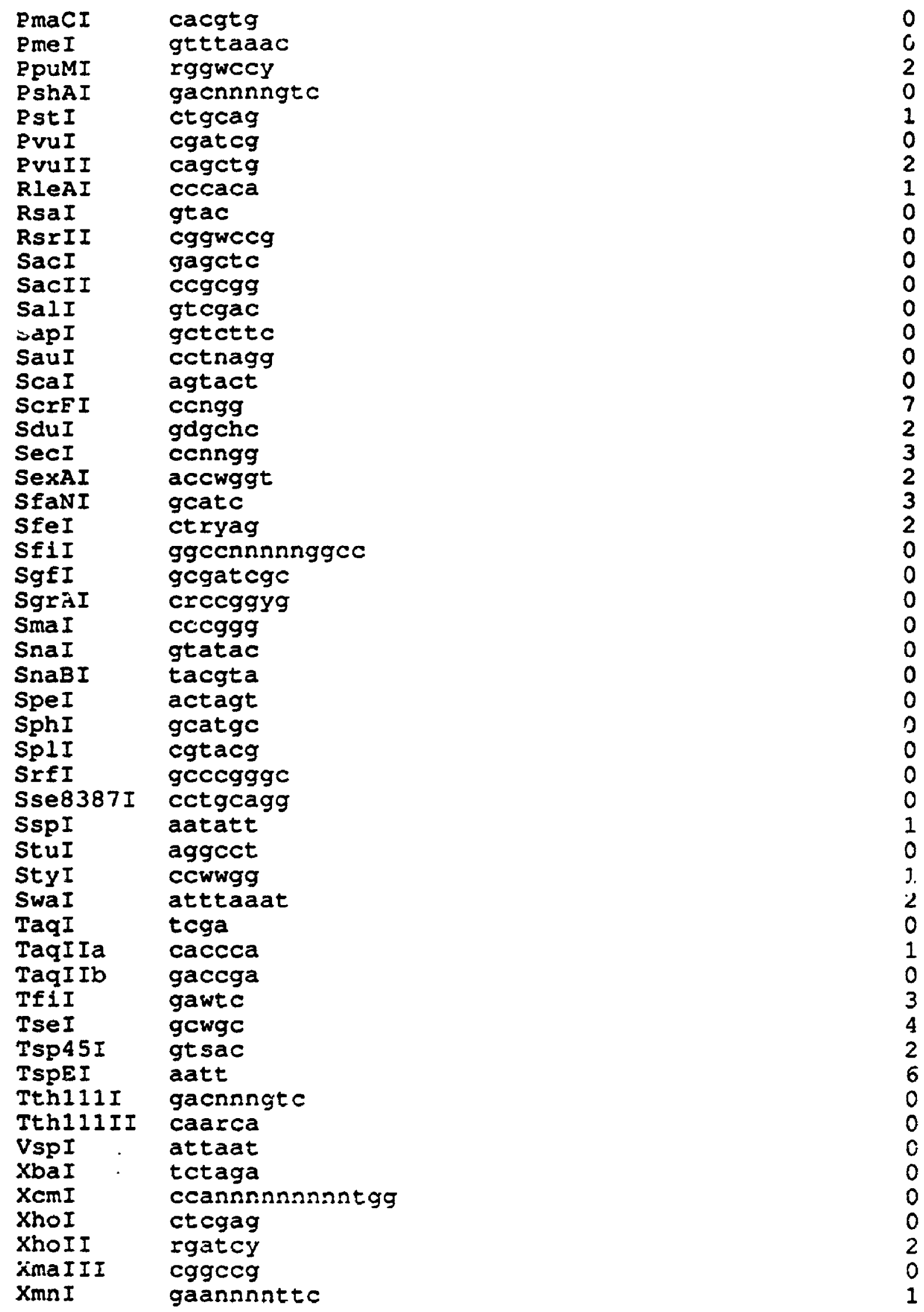


Total

234 


\section{Appendix L}

\section{Human Interferon-Alpha 14 Gene}

\section{L.1 Nucleotide Sequence}

The genomic DNA sequence of the human interferon-alpha 14 gene was retrieved from the Genbank sequence file hsifnal4 (Henco et. al., 1985) (Benson et. al., 1993).

1 gaattgaaa gaaattcta aa3agcccat ggggcaggga aagatgaggt aatactgaaa

61 ataaaagtgg ttgagaaact gctctacacc catgtagaca ggacataaag gaaagccaaa

121 agagaagtag aaaaaaacat gaagacgttc agaaaatgga agctagtatg ttccttattt

181 aagacctatg cacagagcaa ggtcttcaga aaacctacaa cccaaggtte agtgttacce

241 ctcatcaacc agcccagcag catcttcggg attcccaatg geattgccet ttgcttaat

301 gatggecctg gtggtgctca getgcaagtc aagetgetet ctgggetgta atctgtctca

361 aacesacage ctgaataaca ggaggacttt gatgctcatg geacaantga ggagaatctc

421 tcettctcc tgectgaagg acagacatga ctttgaattt ccccaggagg aattgatgg

481 caaccagttc cagaaagetc aagecatctc tglectccat gagatgatge agcagacctt

541 caatctcttc agcacaaaga actcatctge tgettgggat gagacectce tagaaaaatt

601 ctacattgaa ctttccage aaatgaatga cctggaagcc tgtgtgatac aggaggttgg

661 ggtggaagag actcccctga tgaatgagga ctccatcctg gctgtgaaga aatacttcca

721 aagaatcact ctttatctga tggagaagaa atacagecet tgtgectggg aggttgtcag

781 ageagaaatc atgagatcce tctcttttc aacaaacttg craaaangat raaggaggaa

841 ggattgaaaa ctggttcatc atggaaatga ttctcattga claatacatc atctcacact

901 ttcatgagtt cttccattc aaagactcac ttctcctata accascacaa gttgaatcaa

961 aatttcana tgtttcagg agtgtaanga agcatcatgt atacctgtge aggcactagt

1021 cctttacaga tgaccatgct gatgtctect ttcatetatt tattaaata ttatttatt

1081 taactattt tattattaa attatttt atgtaat $\%$ atgtgt 


\section{L.2 Restriction Endonuclease Sites}

The human interferon-alpha 14 gene restriction endonuclease recognition sites are arranged by ascending sequence location.

\begin{tabular}{|c|c|}
\hline \multicolumn{2}{|l|}{ Name } \\
\hline ApoI & \\
\hline TSPEI & \\
\hline APOI & 12 \\
\hline TSpEI & 1 \\
\hline CVIJI & 2 \\
\hline $\mathrm{BglI}$ & 2 \\
\hline MWOI & 2 \\
\hline NCOI & 2 \\
\hline DsaI & 2 \\
\hline styI & 2 \\
\hline SecI & 2 \\
\hline NIaIII & 2 \\
\hline TaqIIa & 8 \\
\hline NlaIII & \\
\hline ACCI & \\
\hline CviJI & 11 \\
\hline NIaIII & 13 \\
\hline MboII & 14 \\
\hline BbVII & 14 \\
\hline $\mathrm{Xmn} I$ & 14 \\
\hline MaeII & 14 \\
\hline AluI & 16 \\
\hline CviJI & 16 \\
\hline MaeI & 16 \\
\hline MseI & 17 \\
\hline CVIRI & 18 \\
\hline StyI & 22 \\
\hline SecI & 22 \\
\hline MaeIII & 23 \\
\hline $\operatorname{Mn} 1 I$ & 24 \\
\hline CviJI & 25 \\
\hline $\mathrm{Bb} v \mathrm{I}$ & 25 \\
\hline$B c g I$ & 25 \\
\hline TséI & 25 \\
\hline FЛU4HI & 25 \\
\hline SEaNI & \\
\hline TEII & 0 \\
\hline HinfI & \\
\hline MseI & \\
\hline aaeIII & \\
\hline AsuI & \\
\hline CViJI & \\
\hline
\end{tabular}




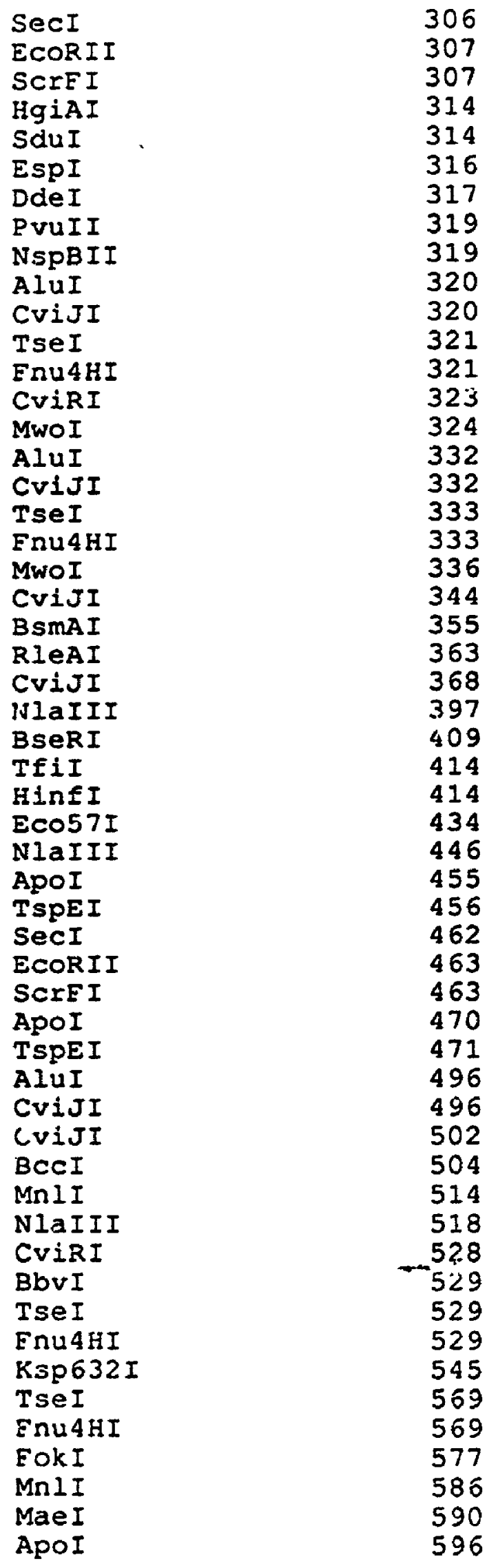




\begin{tabular}{|c|c|}
\hline $\begin{array}{l}\text { TspEI } \\
\text { EcoRII } \\
\text { ScrFI } \\
\text { CviJI } \\
\text { MboII } \\
\text { HinfI } \\
\text { HinfI } \\
\text { BccI } \\
\text { EcoRII } \\
\text { ScrFI } \\
\text { CviJI } \\
\text { MooII } \\
\text { XmnI } \\
\text { TfiI } \\
\text { HinfI } \\
\text { MboII } \\
\text { CviJI } \\
\text { EcoRII } \\
\text { ScrFI } \\
\text { Sec.I } \\
\text { BspHI } \\
\text { NlaIII } \\
\text { XhoII } \\
\text { DpnI/MboI } \\
\text { MnII } \\
\text { CviRI } \\
\text { MseI } \\
\text { BsrI } \\
\text { NIaIII } \\
\text { MslI } \\
\text { XmnI } \\
\text { TfiI } \\
\text { HinfI } \\
\text { MslI } \\
\text { BspHI } \\
\text { NlaIII } \\
\text { HinfI } \\
\text { CjeII } \\
\text { TfiI } \\
\text { HinfI } \\
\text { ApoI } \\
\text { TspEI } \\
\text { SfaNI } \\
\text { NlaIII } \\
\text { SnaI } \\
\text { AccI } \\
\text { BsgI } \\
\text { CviRI } \\
\text { Cac8I } \\
\text { SpeI } \\
\text { MaeI } \\
\text { NlaIII } \\
\text { MsII } \\
\text { BsmAI }\end{array}$ & $\begin{array}{l}597 \\
631 \\
631 \\
637 \\
665 \\
670 \\
689 \\
693 \\
697 \\
697 \\
700 \\
706 \\
709 \\
723 \\
723 \\
745 \\
755 \\
765 \\
765 \\
765 \\
789 \\
790 \\
794 \\
795 \\
799 \\
819 \\
830 \\
850 \\
860 \\
860 \\
864 \\
869 \\
869 \\
897 \\
902 \\
903 \\
924 \\
942 \\
954 \\
954 \\
960 \\
961 \\
992 \\
996 \\
999 \\
999 \\
1007 \\
1008 \\
1009 \\
1015 \\
1016 \\
1035 \\
1035 \\
1044\end{array}$ \\
\hline
\end{tabular}




$\begin{array}{ll}\text { SwaI } & 1062 \\ \text { AhaIII } & 1063 \\ \text { MseI } & 1064 \\ \text { SspI } & 1067 \\ \text { Mse : } & 1080 \\ \text { Swa! } & 1095 \\ \text { AhaI.TI } & 1096 \\ \text { MseI } & 1097 \\ \text { TspEI } & 1100 \\ \text { NlaIII } & 1120\end{array}$

The human interferon-alpha 14 gene restriction endonuclease recognition sites are arranged by enzyme.

$\begin{array}{lr}\text { Name } & \text { Site } \\ \text { AccI } & 94 \\ \text { AcCI } & 999 \\ \text { AhaIII } & 9063 \\ \text { AhaIII } & 10696 \\ \text { AluI } & 1096 \\ \text { AluI } & 161 \\ \text { AIuI } & 320 \\ \text { AIuI } & 332 \\ \text { ApoI } & 496 \\ \text { ApoI } & 1 \\ \text { ApoI } & 12 \\ \text { ApoI } & 455 \\ \text { ApoI } & 470 \\ \text { ApoI } & 596 \\ \text { AsuI } & 960 \\ \text { BbvI } & 304 \\ \text { BbvI } & 257 \\ \text { BbvII } & 529 \\ \text { BccI } & 141 \\ \text { BccI } & 504 \\ \text { BcgI } & 693 \\ \text { BgII } & 257 \\ \text { BseRI } & 25 \\ \text { BsgI } & 409 \\ \text { BsmAI } & 1007 \\ \text { BsmAI } & 355 \\ \text { BspHI } & 1044 \\ \text { BspHI } & 789 \\ \text { BsII } & 902 \\ \text { Cac8I } & 850 \\ \text { CjeI } & 1009 \\ \text { CviJI } & 942 \\ \text { CviJI } & 24 \\ \text { CviJI } & 114 \\ & 161\end{array}$




\begin{tabular}{|c|c|}
\hline $\begin{array}{l}\text { CviJI } \\
\text { CviJI } \\
\text { CviJI } \\
\text { CviJI } \\
\text { CviJI } \\
\text { CviJI } \\
\text { CviJI } \\
\text { CviJI } \\
\text { CviJI } \\
\text { CviJI } \\
\text { CviJI } \\
\text { CviRI } \\
\text { CviRI } \\
\text { CviRI } \\
\text { CviRI } \\
\text { CviRI } \\
\text { DdeI } \\
\text { DpnI/MboI } \\
\text { DsaI } \\
\text { Ecos7I } \\
\text { EcoRII } \\
\text { EcoRII } \\
\text { EcoRII } \\
\text { EcoRII } \\
\text { EcoRII } \\
\text { EspI } \\
\text { Enu4HI } \\
\text { Enu4HI } \\
\text { Enu4HI } \\
\text { Enu4HI } \\
\text { Enu4HI } \\
\text { EokI } \\
\text { HaeIII } \\
\text { HgiAI } \\
\text { HinfI } \\
\text { HinfI } \\
\text { HinfI } \\
\text { HinfI } \\
\text { HinfI } \\
\text { HinfI } \\
\text { HinfI } \\
\text { HinfI } \\
\text { Ksp632I } \\
\text { MaeI } \\
\text { MaeI } \\
\text { MaeI } \\
\text { MaeII } \\
\text { MaeIII } \\
\text { MboII } \\
\text { MboII } \\
\text { MboII } \\
\text { MboII } \\
\text { MnII } \\
\text { MnII } \\
\text { Cvoling }\end{array}$ & $\begin{array}{r}251 \\
304 \\
320 \\
332 \\
344 \\
368 \\
496 \\
502 \\
637 \\
700 \\
755 \\
189 \\
323 \\
528 \\
819 \\
1008 \\
317 \\
795 \\
27 \\
434 \\
307 \\
463 \\
631 \\
j 97 \\
765 \\
316 \\
257 \\
321 \\
333 \\
529 \\
569 \\
577 \\
304 \\
314 \\
270 \\
414 \\
670 \\
689 \\
723 \\
869 \\
924 \\
954 \\
545 \\
163 \\
590 \\
145\end{array}$ \\
\hline
\end{tabular}




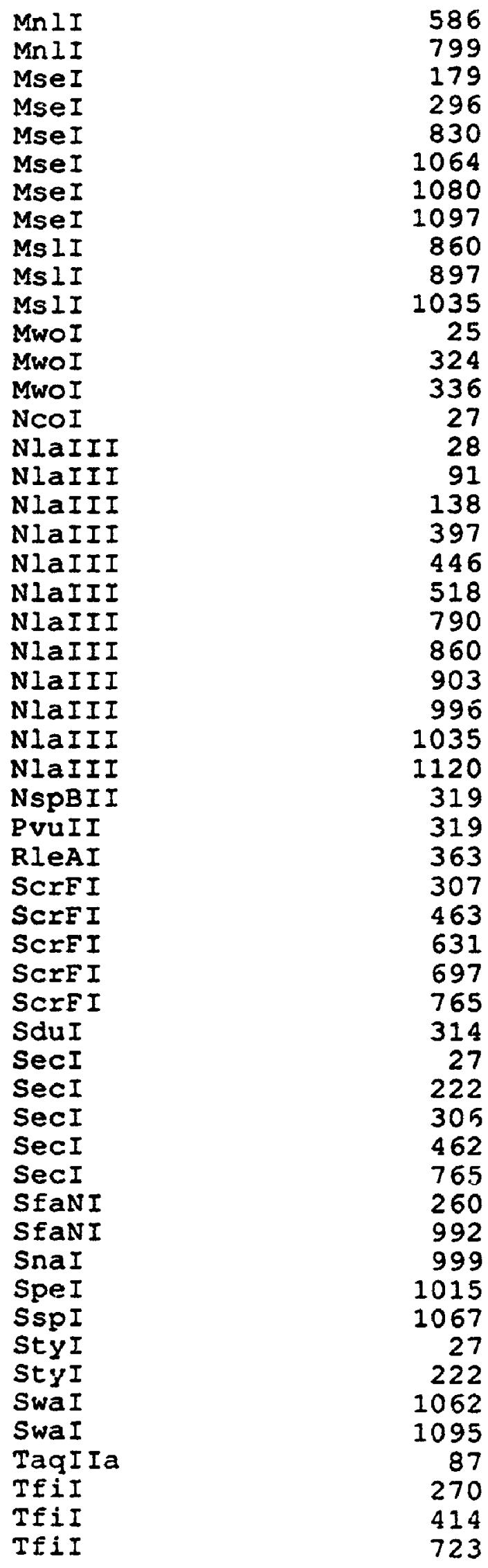




$\begin{array}{lr}\text { TfiI } & 869 \\ \text { TfiI } & 954 \\ \text { TseI } & 257 \\ \text { TseI } & 321 \\ \text { TseI } & 333 \\ \text { TseI } & 529 \\ \text { TseI } & 569 \\ \text { TspEI } & 2 \\ \text { TspEI } & 13 \\ \text { TspEI } & 456 \\ \text { TspEI } & 471 \\ \text { TspEI } & 597 \\ \text { TspEI } & 961 \\ \text { TspEI } & 1100 \\ \text { XhoII } & 794 \\ \text { XmnI } & 141 \\ \text { XmnI } & 709 \\ \text { XmnI } & 864\end{array}$

The human interferon-alpha 14 gene restriction endonuclease recognition sites are summarized by enzyme.

$\begin{array}{llr}\text { Name } & \text { Pattern } & \text { Sites } \\ \text { AatII } & \text { gacgtc } & 0 \\ \text { AccI } & \text { gtmkac } & 2 \\ \text { AciI } & \text { ccgc } & 0 \\ \text { AcII } & \text { aacgtt } & 0 \\ \text { AcyI } & \text { grcgyc } & 0 \\ \text { AfIII } & \text { cttaag } & 0 \\ \text { AflIII } & \text { acrygt } & 0 \\ \text { AgeI } & \text { accggt } & 0 \\ \text { AhaIII } & \text { tttaad } & 2 \\ \text { AluI } & \text { agct } & 4 \\ \text { AlwNI } & \text { cagnnnctg } & 0 \\ \text { ApaI } & \text { gggccc } & 0 \\ \text { ApaBI } & \text { gcannnnntgc } & 0 \\ \text { AnzII } & \text { gtgcac } & 0 \\ \text { ApoI } & \text { raatty } & 6 \\ \text { AscI } & \text { ggcgcgcc } & 0 \\ \text { AsuI } & \text { ggncc } & 1 \\ \text { AsuII } & \text { ttcgaa } & 0 \\ \text { AvaI } & \text { cycgrg } & 0 \\ \text { AvaII } & \text { ggNcc } & 0 \\ \text { AvaIII } & \text { atgcat } & 0 \\ \text { AvIII } & \text { cctagg } & 0 \\ \text { BaeI } & \text { acnnngtayc } & 0 \\ \text { BalI } & \text { tggcca } & 0 \\ \text { BamHI } & \text { ggatcc } & 0 \\ \text { BbvI } & \text { gcagc } & 2\end{array}$




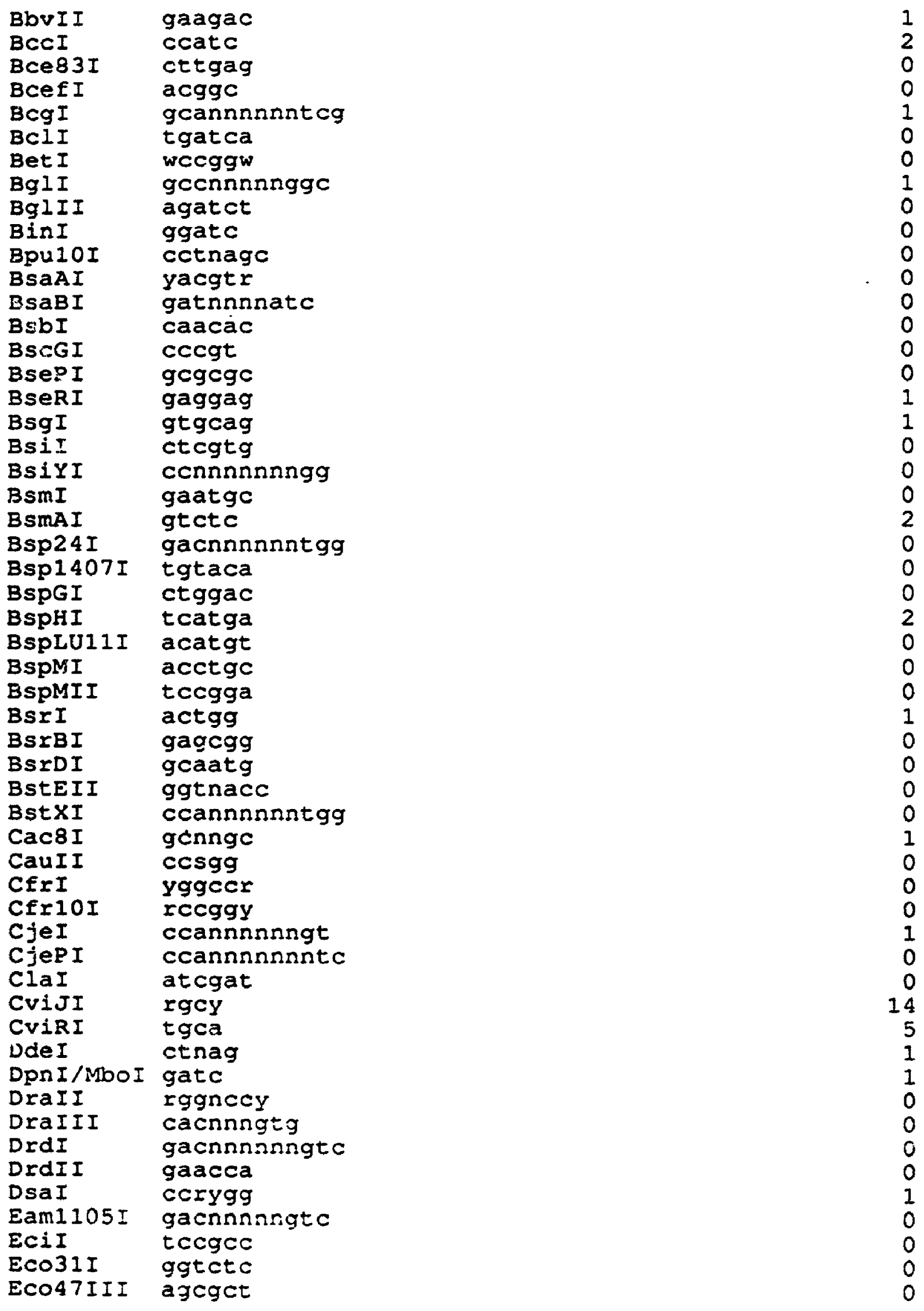




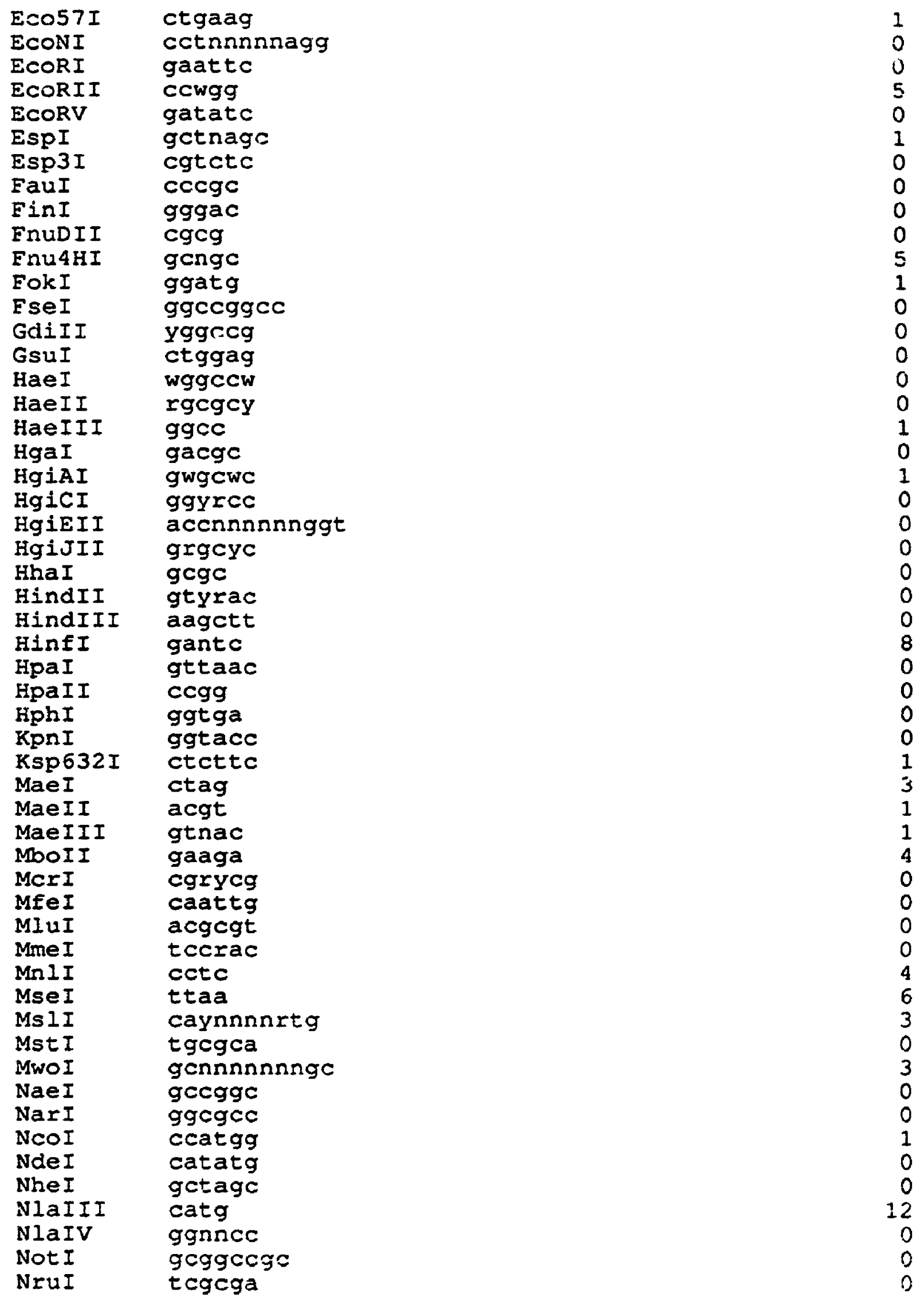




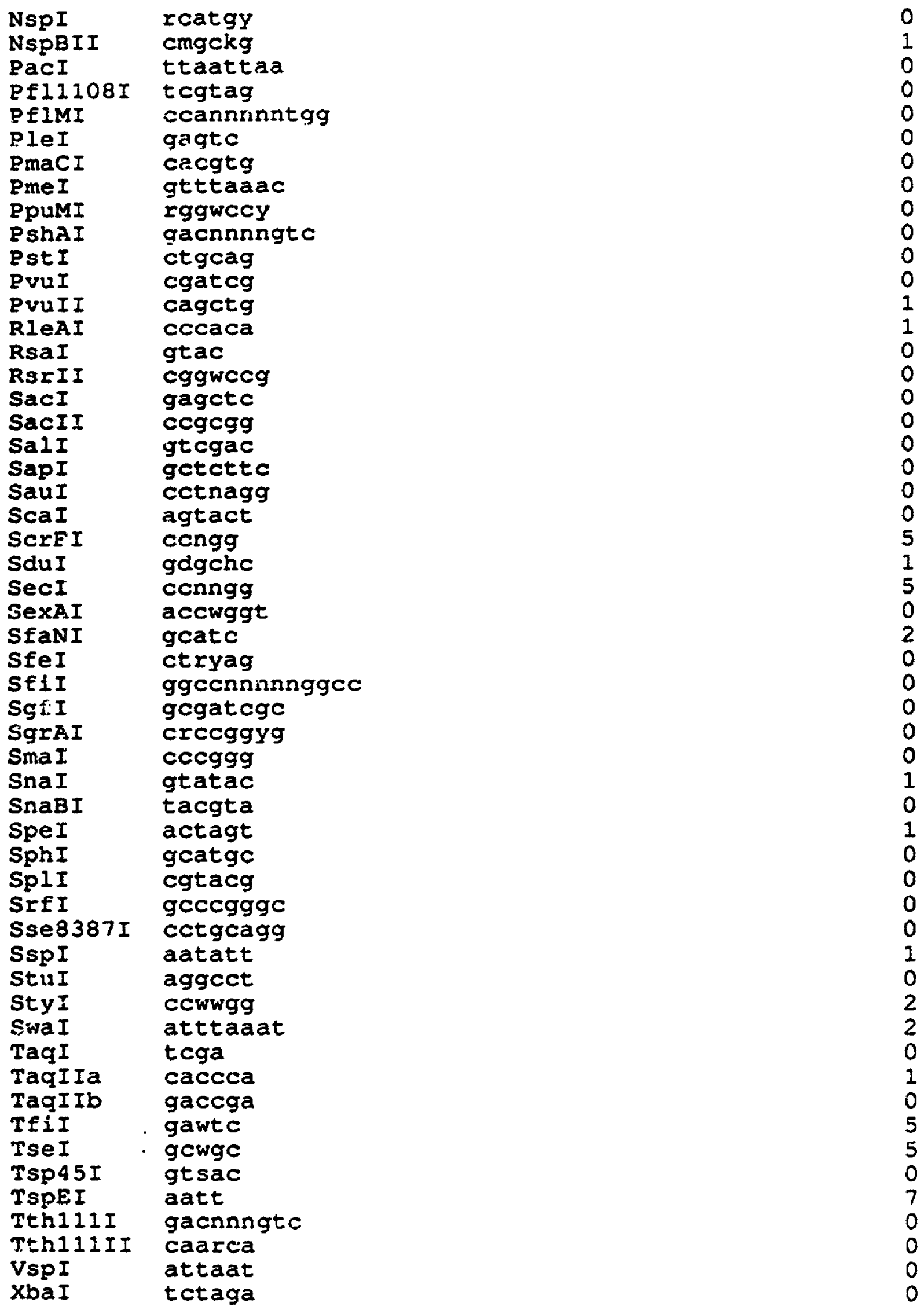


XcmI ccannnnnnnnntgg

XhoI

ctcgag

XhoII

rgatcy

XmaIII

cggcog

1

$X m n I$

gaannnnttc

0

3

Total 


\title{
Appendix $\mathbf{M}$
}

\section{Human Interferon-Alpha 16 Gene}

\author{
M.1 Nucleotide Sequence
}

The genomic DNA sequence of the human interferon-alpha 16 gene was retrieved from the Genbank sequence file hsifnal6 (Henco et. al., 1985) (Benson et. al., 1993).

1 aattacacta aaagttattc cagaaatata cttatcaaat taagttatat gtcaacagct

61 tttaaactta gattttagtt taactttct gtcattctta actttacatt gaathaaaag

121 agcaaacttt atagtttta tctgtgaagt agaggtatac gtagtataca taaatagata

181 tgccaaatct gtgttattaa aattcatga agattccaat tagtaaaaaa taccataaaa

241 gtcttgagt gcaggggaaa aataggcaat gatgaacaaa atgaaaaca tatgtaaaca

301 catgtagaga gtgcataaag aaagcaaaan cagagataga aagtaaaacc agggcattta

361 gaaaatggaa attagtatgt tcactatta agaactatgc acagagcaaa gtcttcagaa

421 aacctagagg ccanggttca agtttaccca tctccagtag cctagcaata tttgcaacat

481 cccaatggcc ctgtccttt ctttactgat ggccgtgctg gtgctcagct acaaatccat

541 ctgttctctg ggctgtgatc tgcctcagac tcacagectg ggtaatagga gggccttgat

601 actcctggca caaatgggaa gaatctctca tttctcctge ctgaaggaca gatatgattt

661 cggattcccc caggaggtgt ttgatggcaa ccagttccag aaggctcaag ccatctctge

721 cttccatgag atgatccagc agaccttcaa tetcttcagc acaaaggatt catctgctgc

781 ttgggatgag accctcctag acaaattcta cattgaactt ttccagcaac tgaatgacct

841 agaagectgt zitgacacagg aggttggggt ggaagagatt gecetgatga atgaggactc

901 catcctggct gtgaggaaat acttcaaag aatcactctt tatctgatgg ggaagaaata

961 cagcccttgt gecigggagg ttgtcagage agaaatcatg agatcettct cttttcaac

1021 aanctgc a aaggattaa gaaggaagga ttgaaactc attcancatg gaaatgatcc

1081 tcattgatta atacatc atc tcacacttc atgagttctt ccattcaaa gac 


\section{M.2 Restriction Endonuclease Sites}

The human interferon-alpha 16 gene restriction endonuclease recognition sites are arranged by ascending sequence location.

\begin{tabular}{|c|c|}
\hline Name & Site \\
\hline TSpEI & 1 \\
\hline TspEI & 38 \\
\hline MseI & 40 \\
\hline HindII & 51 \\
\hline AluI & 57 \\
\hline CviJI & 57 \\
\hline AhaIII & 61 \\
\hline MseI & 62 \\
\hline DdeI & 67 \\
\hline MseI & 80 \\
\hline MseI & 98 \\
\hline SnaI & 155 \\
\hline AccI & 155 \\
\hline SnaBI & 158 \\
\hline BsaAI & 158 \\
\hline MaeII & $\begin{array}{l}159 \\
164\end{array}$ \\
\hline $\begin{array}{l}\text { SnaI } \\
\text { AccI }\end{array}$ & $\begin{array}{l}104 \\
164\end{array}$ \\
\hline MseI & 197 \\
\hline ApoI & 200 \\
\hline TspeI & 201 \\
\hline BspHI & 205 \\
\hline NlaIII & 206 \\
\hline MboII & 209 \\
\hline TfiI & 212 \\
\hline HInEI & 212 \\
\hline TspEI & 218 \\
\hline BsgI & 249 \\
\hline CVIRI & 250 \\
\hline Bs rDI & 266 \\
\hline NdeI & 289 \\
\hline BspLU11I & 300 \\
\hline AEIIII & 300 \\
\hline NspI & 300 \\
\hline NlaIII & 301 \\
\hline CVIRI & 312 \\
\hline ECORII & 349 \\
\hline ScrEI & 349 \\
\hline SecI & 349 \\
\hline TSPEI & 370 \\
\hline MseI & 388 \\
\hline CVIRI & 98 \\
\hline
\end{tabular}




\begin{tabular}{|c|c|}
\hline $\begin{array}{l}\text { MaeI } \\
\text { HaeI } \\
\text { HaeIII } \\
\text { CviJI } \\
\text { StyI } \\
\text { SecI } \\
\text { BccI } \\
\text { CjeI } \\
\text { CviJI } \\
\text { MaeI } \\
\text { ApaBI } \\
\text { MwoI } \\
\text { SspI } \\
\text { CviRI } \\
\text { MsII } \\
\text { HaeIII } \\
\text { AsuI } \\
\text { CviJI } \\
\text { GdiII } \\
\text { CfrI } \\
\text { HaeIII } \\
\text { CviJI } \\
\text { HgiAI } \\
\text { SduI } \\
\text { EspI } \\
\text { DdeI } \\
\text { AluI } \\
\text { CviJI } \\
\text { BccI } \\
\text { CjePI } \\
\text { XcmI } \\
\text { CviJI } \\
\text { DpnI/MboI } \\
\text { MnII } \\
\text { DdeI } \\
\text { HinfI } \\
\text { CviJI } \\
\text { EcoRII } \\
\text { ScrEI } \\
\text { SecI } \\
\text { DraII } \\
\text { AsuI } \\
\text { HaeIII } \\
\text { CviJI } \\
\text { EcoRII } \\
\text { ScrFI } \\
\text { MboII } \\
\text { TfiI } \\
\text { HinfI } \\
\text { Eco57I } \\
\text { TEiI } \\
\text { HinfI } \\
\text { BsiYI } \\
\text { SecI } \\
\text { Sed }\end{array}$ & $\begin{array}{l}424 \\
428 \\
429 \\
429 \\
431 \\
431 \\
448 \\
448 \\
459 \\
462 \\
465 \\
465 \\
467 \\
473 \\
478 \\
487 \\
487 \\
487 \\
510 \\
510 \\
511 \\
511 \\
521 \\
521 \\
523 \\
524 \\
527 \\
527 \\
537 \\
537 \\
537 \\
551 \\
557 \\
563 \\
564 \\
568 \\
575 \\
577 \\
577 \\
577 \\
590 \\
591 \\
592 \\
592 \\
604 \\
604 \\
618 \\
621 \\
621 \\
641 \\
663 \\
663 \\
667 \\
669\end{array}$ \\
\hline
\end{tabular}




\begin{tabular}{|c|c|}
\hline 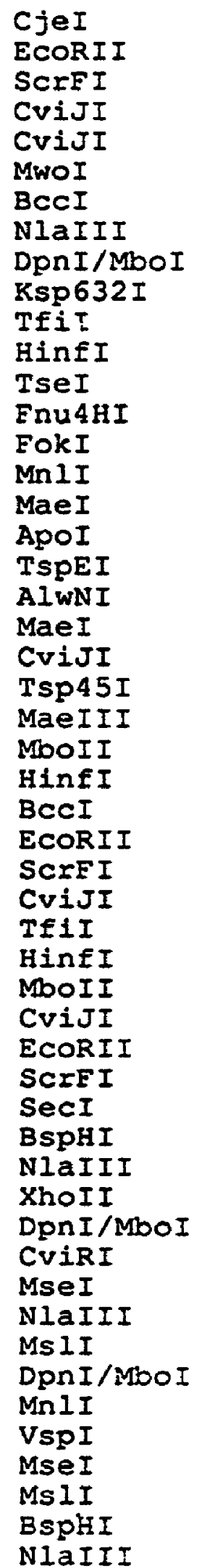 & $\begin{array}{l}670 \\
670 \\
670 \\
703 \\
709 \\
710 \\
711 \\
725 \\
733 \\
752 \\
767 \\
767 \\
776 \\
776 \\
784 \\
793 \\
797 \\
803 \\
804 \\
824 \\
839 \\
844 \\
851 \\
851 \\
872 \\
896 \\
900 \\
904 \\
904 \\
907 \\
930 \\
930 \\
952 \\
962 \\
972 \\
972 \\
972 \\
996 \\
997 \\
1001 \\
1002 \\
1026 \\
1037 \\
1067 \\
1067 \\
1076 \\
1079 \\
1087 \\
1088 \\
1104 \\
1109 \\
1110\end{array}$ \\
\hline
\end{tabular}


The human interferon-alpha 16 gene restriction endonuclease recognition sites are arranged by enzyme.

$\begin{array}{lr}\text { Name } & \text { Site } \\ \text { AccI } & \\ \text { ACCI } & 155 \\ \text { AfIIII } & 164 \\ \text { AhaIII } & 300 \\ \text { AluI } & 61 \\ \text { AluI } & 57 \\ \text { AIwNI } & 527 \\ \text { ApaBI } & 824 \\ \text { ApoI } & 465 \\ \text { ApoI } & 200 \\ \text { AsuI } & 803 \\ \text { AsuI } & 487 \\ \text { BCCI } & 591 \\ \text { BCCI } & 448 \\ \text { BCCI } & 537 \\ \text { BCCI } & 711 \\ \text { BsaAI } & 900 \\ \text { BsgI } & 158 \\ \text { BsiYI } & 249 \\ \text { BspHI } & 667 \\ \text { BspHI } & 205 \\ \text { BspHI } & 996 \\ \text { BspIUIII } & 1109 \\ \text { BsrDI } & 300 \\ \text { CfrI } & 266 \\ \text { CjeI } & 510 \\ \text { CjeI } & 526 \\ \text { CjePI } & \end{array}$




$\begin{array}{lr}\text { DdeI } & 67 \\ \text { DdeI } & 524 \\ \text { DdeI } & 564 \\ \text { DpnI/MboI } & 557 \\ \text { DpnI/MboI } & 733 \\ \text { DpnI/MboI } & 1002 \\ \text { DpnI/MboI } & 1076 \\ \text { DraII } & 590 \\ \text { Eco57I } & 641 \\ \text { EcoRII } & 349 \\ \text { EcoRII } & 577 \\ \text { EcoRII } & 604 \\ \text { EcoRII } & 670 \\ \text { EcoRII } & 904 \\ \text { EcoRII } & 972 \\ \text { EspI } & 523 \\ \text { Enu4HI } & 776 \\ \text { EokI } & 784 \\ \text { GdiII } & 510 \\ \text { HaeI } & 428 \\ \text { HaeIII } & 429 \\ \text { HaeIII } & 487 \\ \text { HaeIII } & 69 \\ \text { HaeIII } & 397 \\ \text { HgiAI } & 1037 \\ \text { HindII } & \end{array}$




$\begin{array}{lr}\text { MseI } & 1088 \\ \text { MsII } & 478 \\ \text { MsII } & 1067 \\ \text { MsII } & 1104 \\ \text { MwoI } & 465 \\ \text { MwoI } & 710 \\ \text { NceI } & 289 \\ \text { NlaIII } & 206 \\ \text { NlaIII } & 301 \\ \text { NlaIII } & 725 \\ \text { NlaIII } & 997 \\ \text { NlaIII } & 1067 \\ \text { NlaIII } & 110 \\ \text { NspI } & 300 \\ \text { ScrEI } & 349 \\ \text { ScrEI } & 577 \\ \text { ScrEI } & 604 \\ \text { ScrEI } & 670 \\ \text { ScrEI } & 904 \\ \text { ScrEI } & 972 \\ \text { SduI } & 521 \\ \text { SecI } & 349 \\ \text { SecI } & 537 \\ \text { SecI } & 1001 \\ \text { SecI } & \end{array}$


The human interferon-alpha 16 gene restriction endonuclease recognition sites are summarized by enzyme.

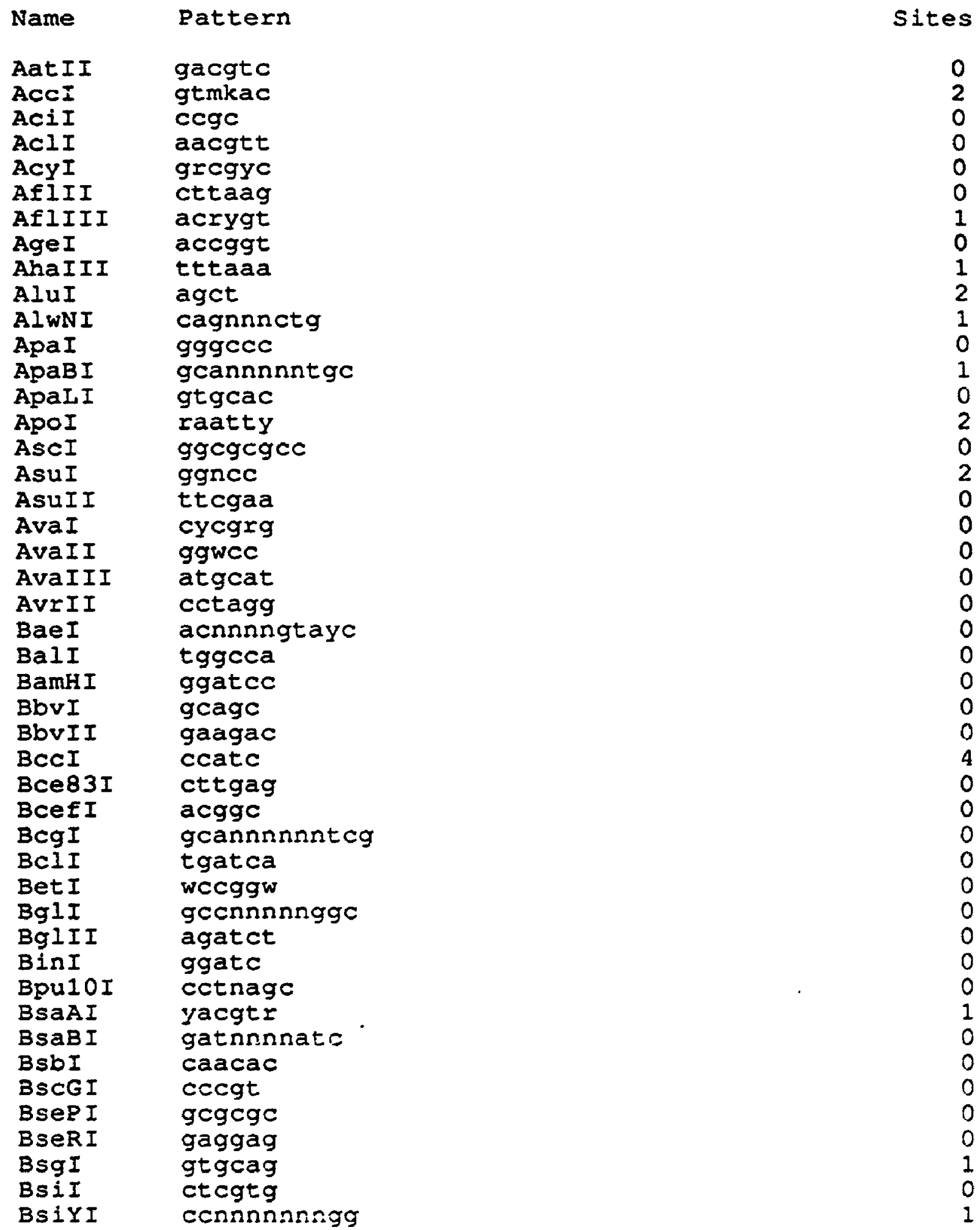




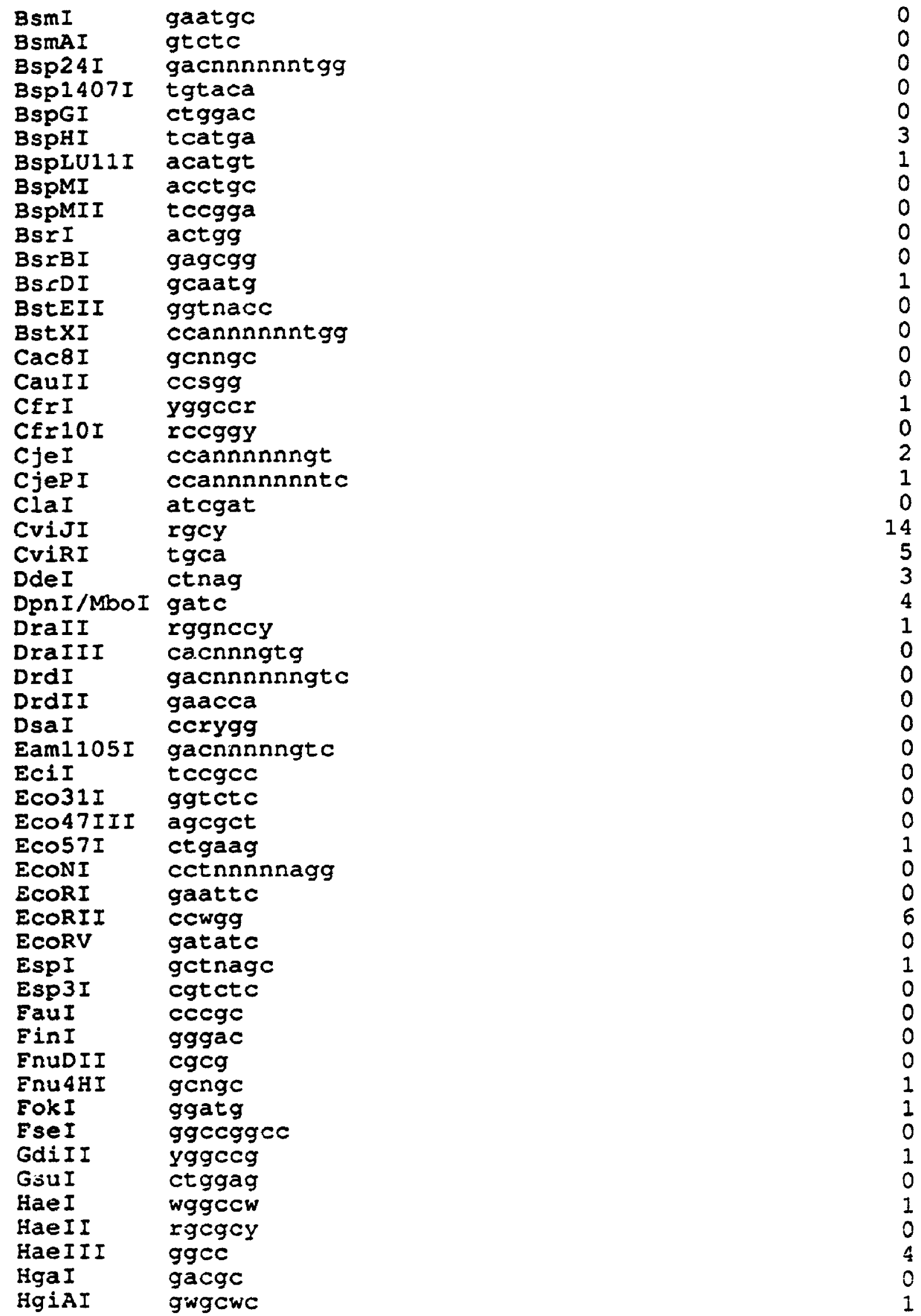




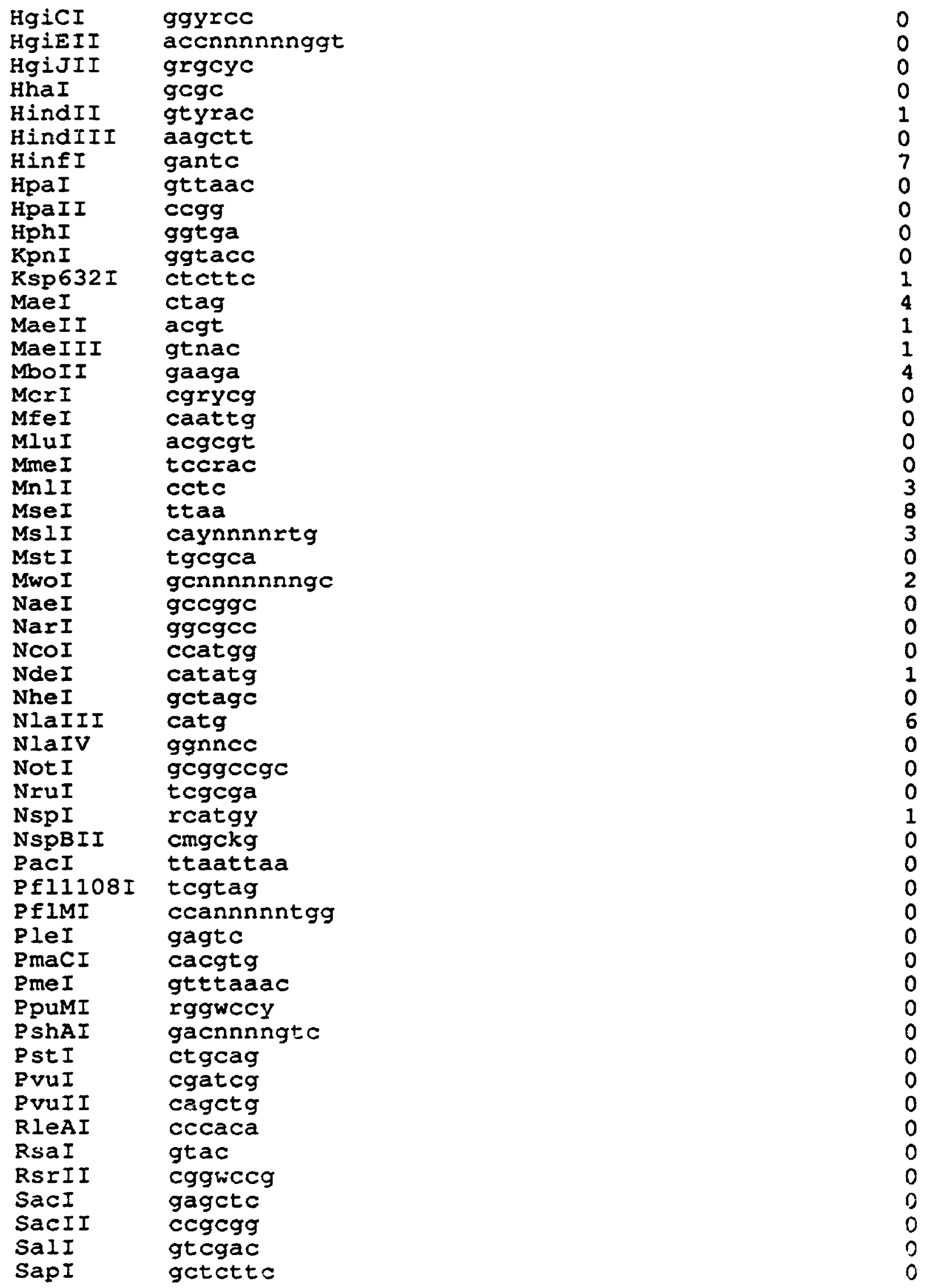




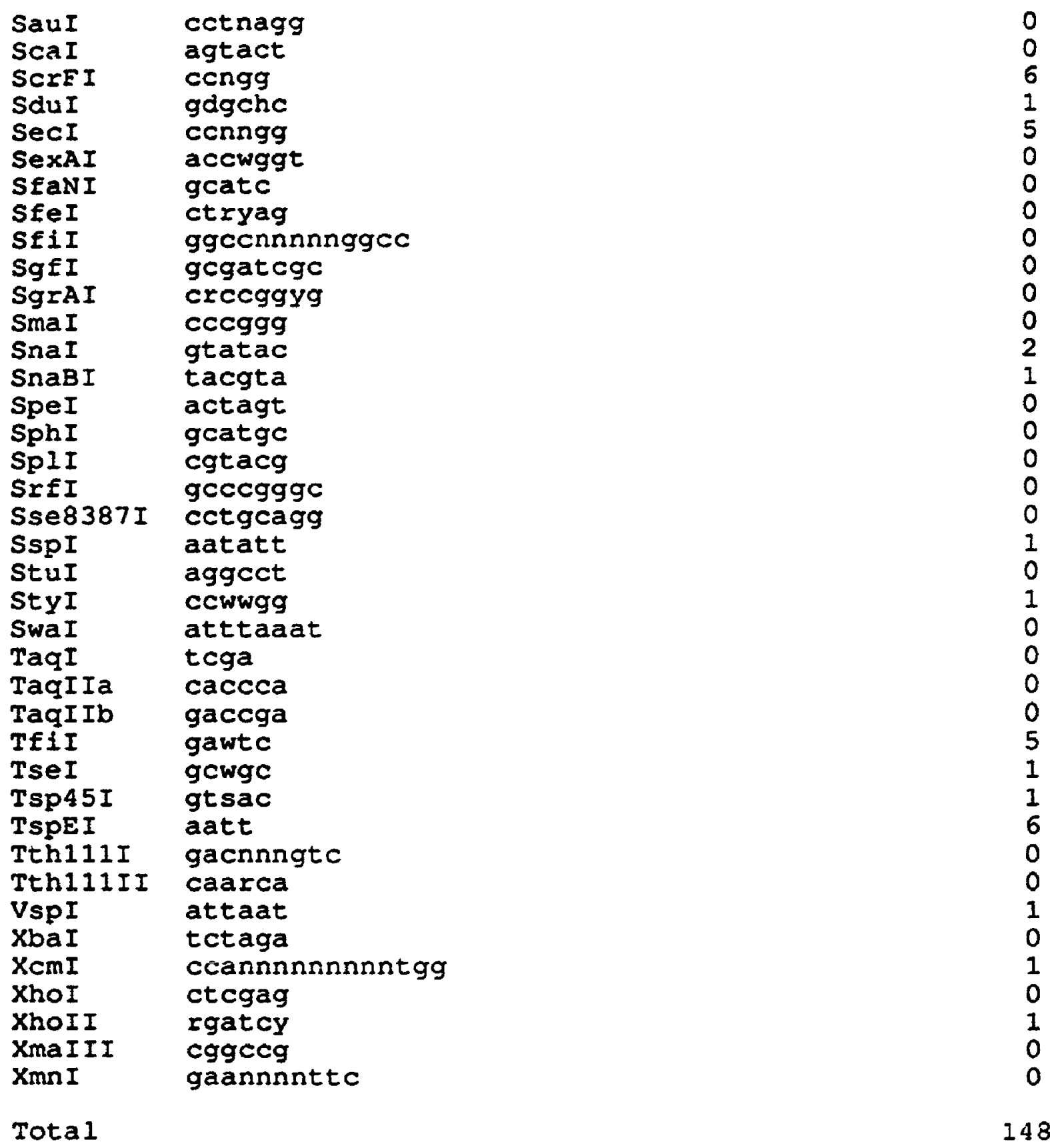




\section{Appendix $\mathbf{N}$}

\section{Human Interferon-Alpha 17 Gene}

\section{N.1 Nucleotide Sequence}

The genomic DNA sequence of the human interferon-alpha 17 gene was retrieved from the Genbank sequence file hsifd2 (Lawn et. al., 1981a) (Benson et. al., 1993).

1 agcttcatac actaagagaa aaatttaaa aaattattga ttcttattt caggagtttt

61 gaatgatcag gtatgtaatt atattcatat tattaatgtg tatttatata gattttatt

121 ttgcacaggt acttgatac aaaattraca tgaacaaatt acactaaaag ttatttcaca

181 aatatactta tcaagttaag ttaaatgcaa tagcttaaa cttagattt aattraactt

241 tttctatcat cattctttac attaaataaa aaaagcaaac tttatagttt ttatctataa

301 agtagaggta tacatagtat acataaatac atatgccaaa tctgtgttat taaaacttca

361 tgaagattc gattacaaaa aaataccgta aaagacttg agtgcagaag aaaaatgggc

421 aatgatgaaa aacaatgaaa aacattctta aacacatgta gagagtgcaa aaaganagea

48: aaaacagaca tagaaagtaa aactagggca ttagaaaat ggaaattagt atgttcacta

541 tttaaggcct atgcacagag caaagtcttc agaaaaccta gaggccaang ttcaaggtta

601 cccatctcaa gtagcctagc aacatttgca acatcccaat ggccetgtcc tttctttac

661 tgatggccgt getggtgetc agctacaaat ccatctgttc tctaggctgt gatctgcctc

721 agacceacag cctgggtaat aggagggect tgatactcct ggcacaaatg ggaagaatct

781 ctccttrctc ctgcctgaag gacagacctg actttggact tccccaggag gagtttgatg

841 gcaaccagtt ccagaagact caagccatct ctgtcctcca tgagatgatc cagcagacct

901 tcaatctctt cagcacagag gactcatctg ctgcttggga acagagcctc ctagaaaaat

961 ttrccactga actttaccag caactgaata acctggaagc atgtgtgata caggaggttg

1021 ggatggaaga gactccctg atgaatgagg actccatcct ggctgtgagg aaatacttcc

1081 aaagaatcac tctttatcta acagagaaga aatacagcce ttgtgectgg gaggttgtca

1141 gagcagaaat catgagatct ctctctttt caacaaactt gcaaaaaata ttaaggagga

1201 aggattgaaa actggttcaa catggcaatg atcetgattg actaatacat tatctcacac

1261 tttcatgagt tcctccatt caaagactca cttctataac caccacgagt tgaatcaaaa

1321 tttcaaatg tttcagcag tgtaaagaag cgtcgtgtat acctgtgcag gcactagtac

1381 tttacagatg accatgctga tgtctctgtt catctatta ttiaaatatt tattraatta

1441 tttttaagat ttaaattatt ttttatgta atatcatgtg taccttaca ttgtggtgaa

1501 tgtaacaata tatgttcttc atattragce aatatattaa tttcctttt cattalaattt

1561 ttactataca aaatttcttg tgtttgttta ttctttaaga taaantgtcg aggctgactt

1621 tacaacctga cttaaanatn tatgatttaa ttangttatc tatcataalt ttattcangt

1681 tattaaaaa acattttct gttactggtt atatgttgce ttcaagatat aałagtgaac

1741 atanaatata co ztcctgt tetcttgtat cttgatttt tgtcaggai:a gaaatctaa

1801 aacaataata atgctgaitt aatitcngtg atgctaactg ctataatgtg aggaagtaaa

1861 atacaatgaa ttc 
N.2 Restriction Endonuclease Sites

The human interferon-alpha 17 gene restriction endonuclease recognition sites are arranged by ascending sequence location.

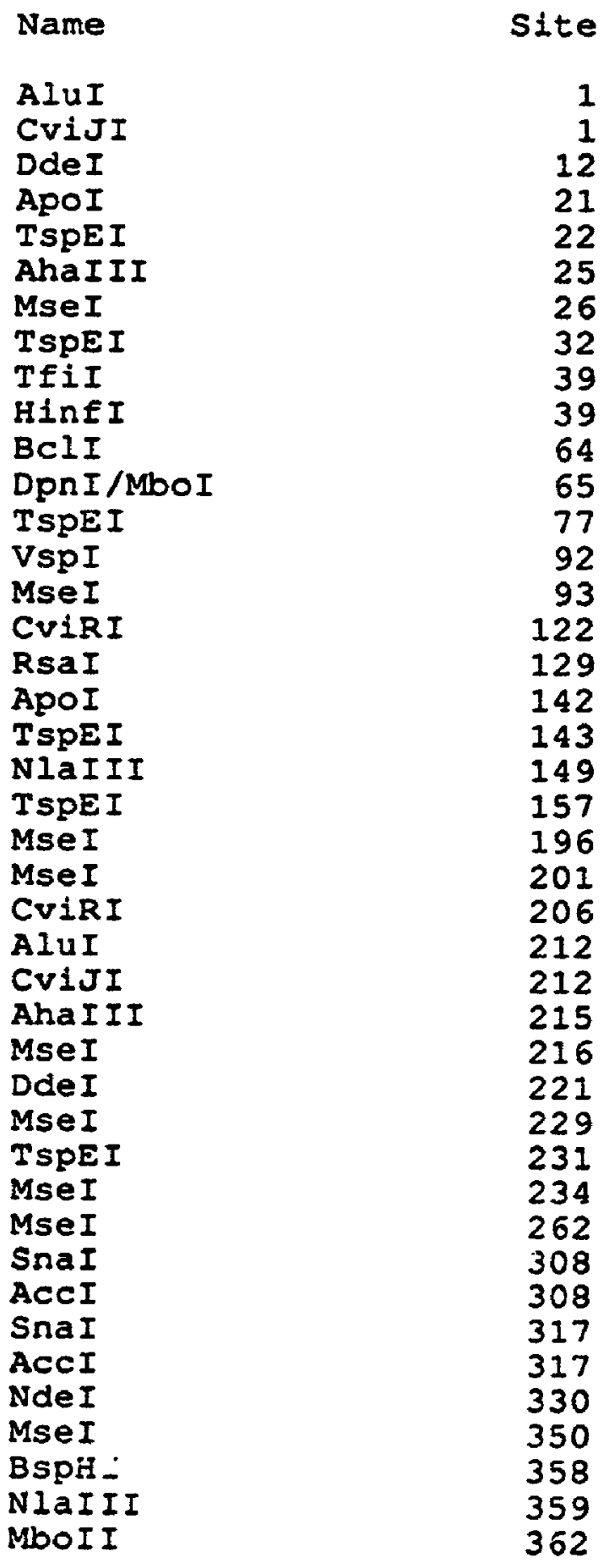




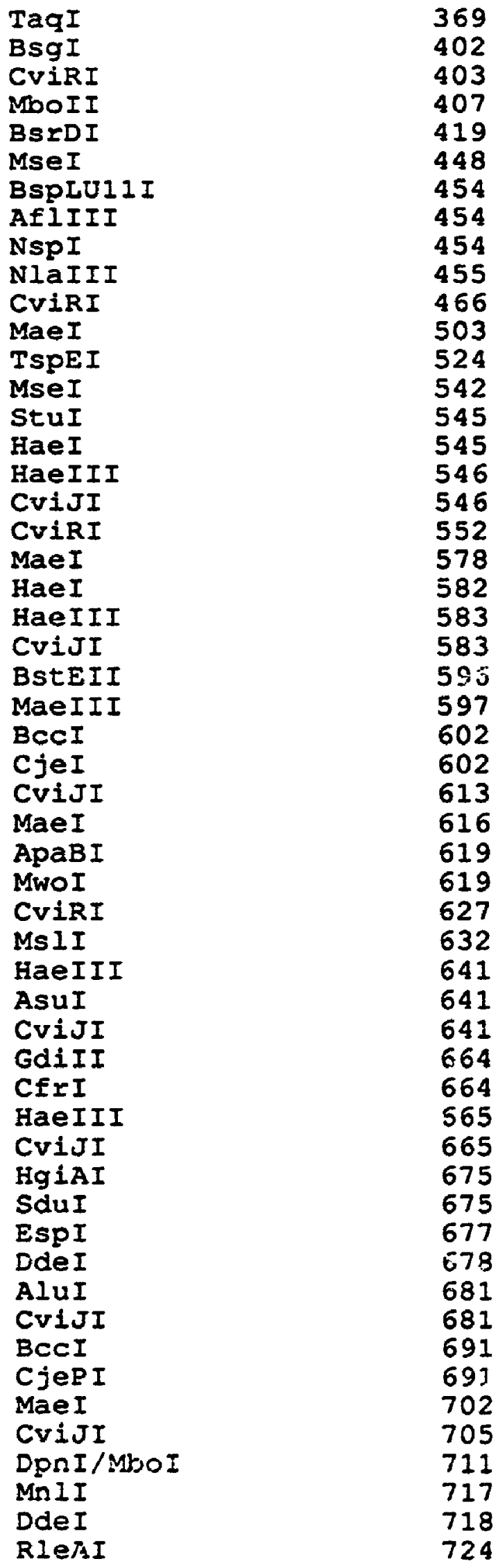




\begin{tabular}{|c|c|}
\hline 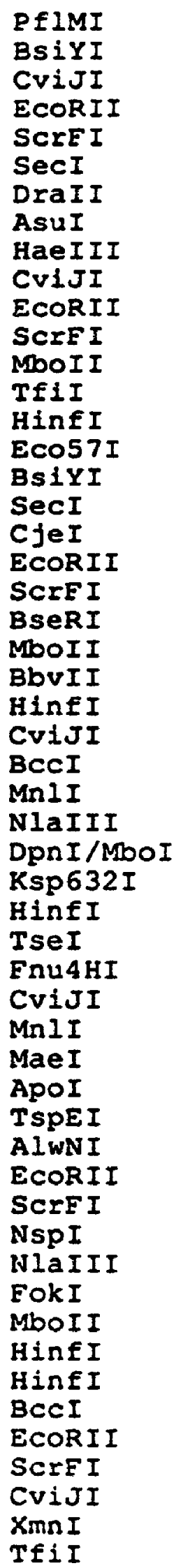 & $\begin{array}{r}725 \\
725 \\
729 \\
731 \\
731 \\
731 \\
744 \\
745 \\
746 \\
746 \\
758 \\
758 \\
772 \\
775 \\
775 \\
795 \\
807 \\
823 \\
824 \\
824 \\
824 \\
828 \\
854 \\
856 \\
857 \\
863 \\
865 \\
875 \\
879 \\
887 \\
906 \\
921 \\
930 \\
930 \\
945 \\
947 \\
951 \\
957 \\
958 \\
978 \\
992 \\
992 \\
999 \\
1000 \\
1021 \\
1026 \\
1031 \\
1050 \\
1054 \\
1058 \\
1058 \\
1061 \\
1084\end{array}$ \\
\hline
\end{tabular}




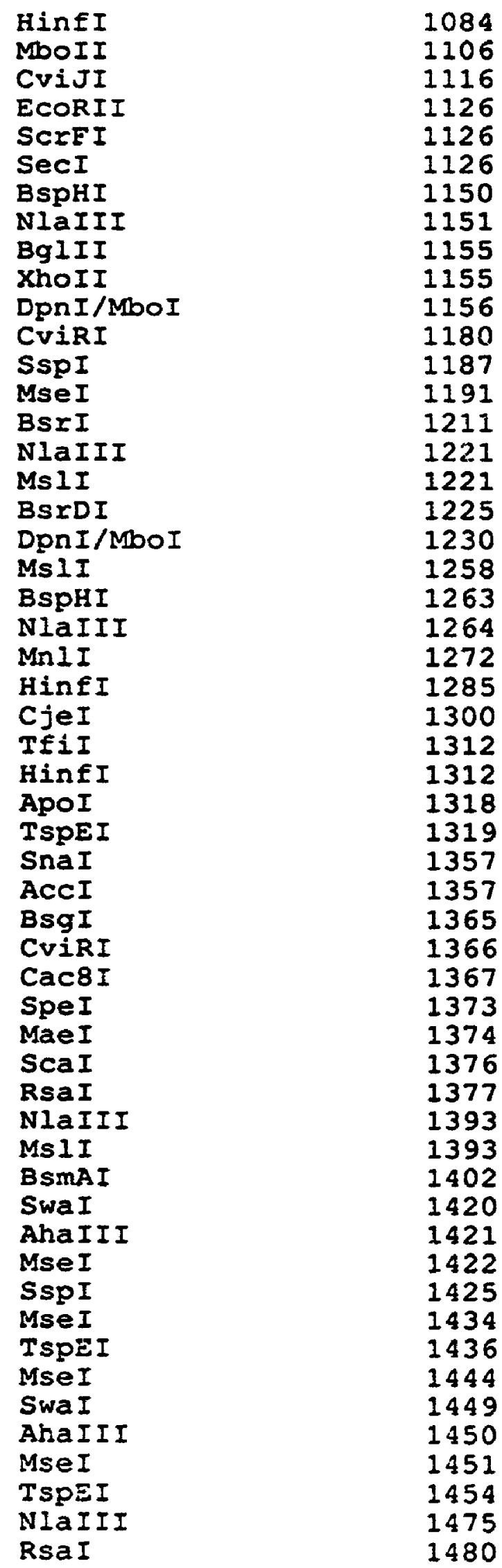




$\begin{array}{ll}\text { MsII } & 1489 \\ \text { HphI } & 1495 \\ \text { MaeIII } & 1502 \\ \text { CviJI } & 1527 \\ \text { VspI } & 1536 \\ \text { MseI } & 1537 \\ \text { TspEI } & 1539 \\ \text { MseI } & 1553 \\ \text { ApoI } & 1555 \\ \text { TspEI } & 1556 \\ \text { ApoI } & 1571 \\ \text { TspEI } & 1572 \\ \text { MseI } & 1595 \\ \text { TaqI } & 1608 \\ \text { CViJI } & 1612 \\ \text { MseI } & 1632 \\ \text { MseI } & 1647 \\ \text { PacI } & 1547 \\ \text { TspEI } & 1649 \\ \text { MseI } & 1651 \\ \text { TspEI } & 1667 \\ \text { MseI } & 1683 \\ \text { MaeIII } & 1701 \\ \text { BsII } & 1704 \\ \text { MaeII } & 1733 \\ \text { AlwNI } & 1751 \\ \text { TspEI } & 1817 \\ \text { VspI } & 1818 \\ \text { MseI } & 1819 \\ \text { EcoRI } & 1868 \\ \text { ApoI } & 1868 \\ \text { TspEI } & 1869\end{array}$

The human interferon-alpha 17 gene restriction endonuclease recognition sites are arranged by enzyme.

$\begin{array}{lr}\text { Name } & \text { site } \\ \text { AccI } & 308 \\ \text { AccI } & 317 \\ \text { AccI } & 1357 \\ \text { AfIIII } & 454 \\ \text { AhaIII } & 25 \\ \text { AhaIII } & 215 \\ \text { AhaIII } & 1421 \\ \text { AhaIII } & 1450 \\ \text { AluI } & 1 \\ \text { AluI } & 212 \\ \text { AluI } & 681 \\ \text { AlwNI } & 978\end{array}$




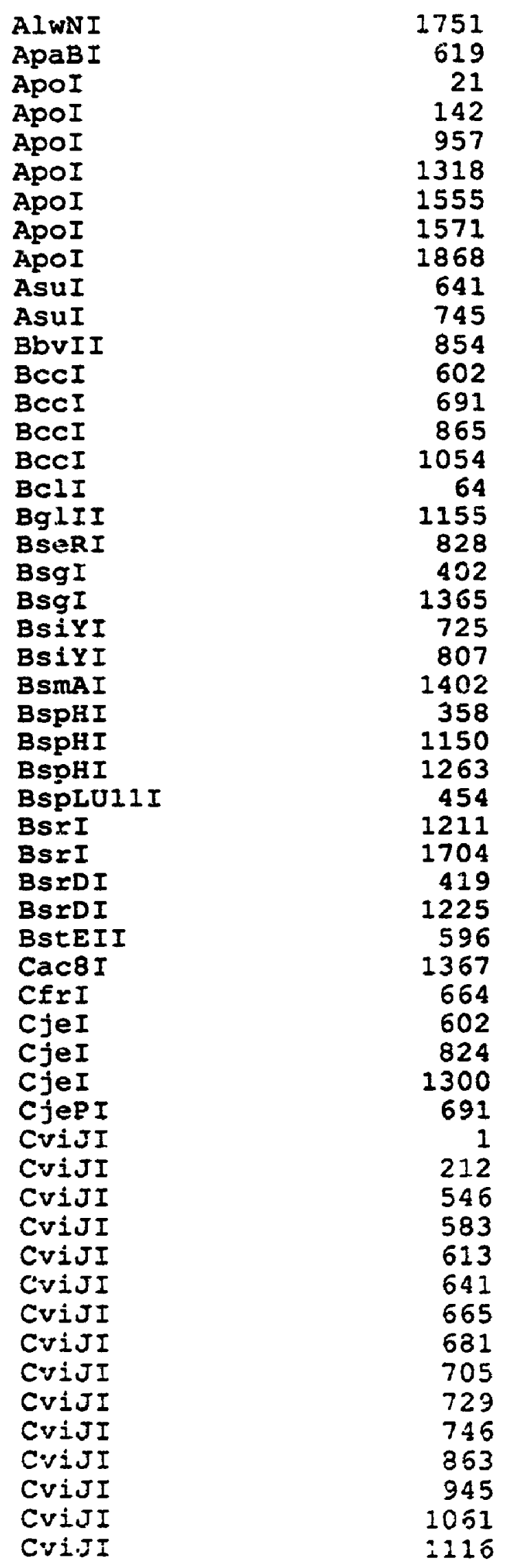




$\begin{array}{lr}\text { CviJI } & 1527 \\ \text { CviJI } & 1612 \\ \text { CviRI } & 122 \\ \text { CviRI } & 206 \\ \text { CviRI } & 403 \\ \text { CviRI } & 466 \\ \text { CviRI } & 552 \\ \text { CviRI } & 627 \\ \text { CviRI } & 1180 \\ \text { CviRI } & 1366 \\ \text { DdeI } & 12 \\ \text { DdeI } & 221 \\ \text { DdeI } & 678 \\ \text { DdeI } & 718 \\ \text { DpnI/MboI } & 65 \\ \text { DpnI/MboI } & 711 \\ \text { DpnI/MboI } & 887 \\ \text { DpnI/MboI } & 1156 \\ \text { DpnI/MboI } & 1230 \\ \text { DraII } & 744 \\ \text { Eco5TI } & 795 \\ \text { EcoRI } & 1868 \\ \text { EcoRII } & 731 \\ \text { ECoRII } & 758 \\ \text { EcoRII } & 824 \\ \text { EcoRII } & 503 \\ \text { EcoRII } & 578 \\ \text { EcoRII } & 616 \\ \text { EspI } & \end{array}$




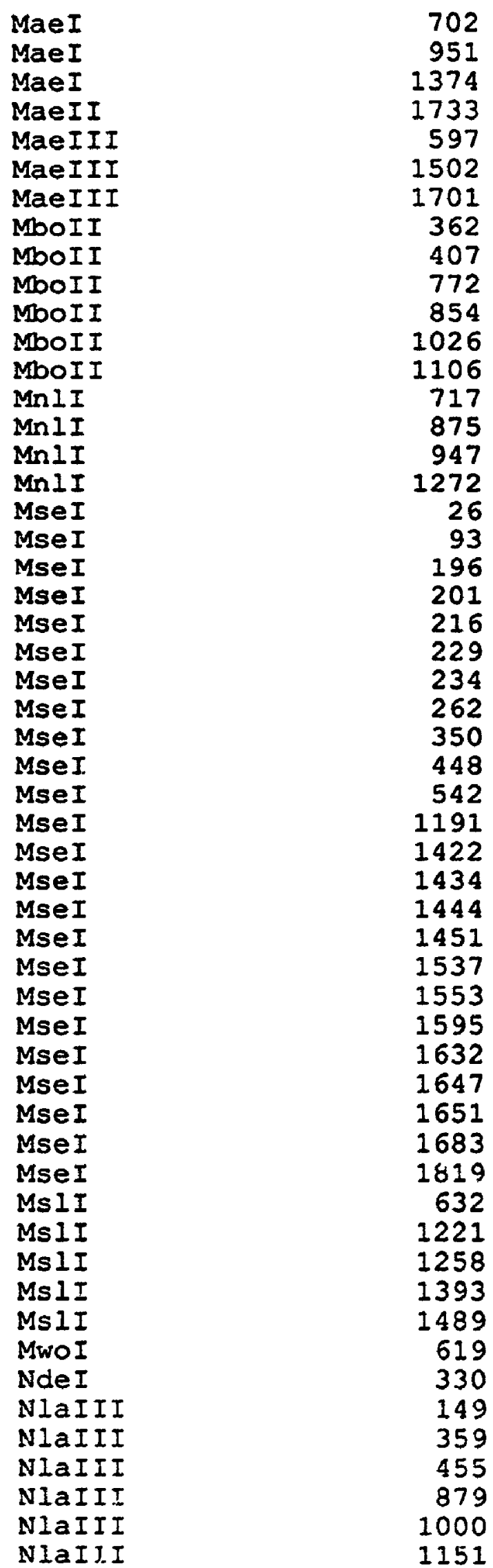




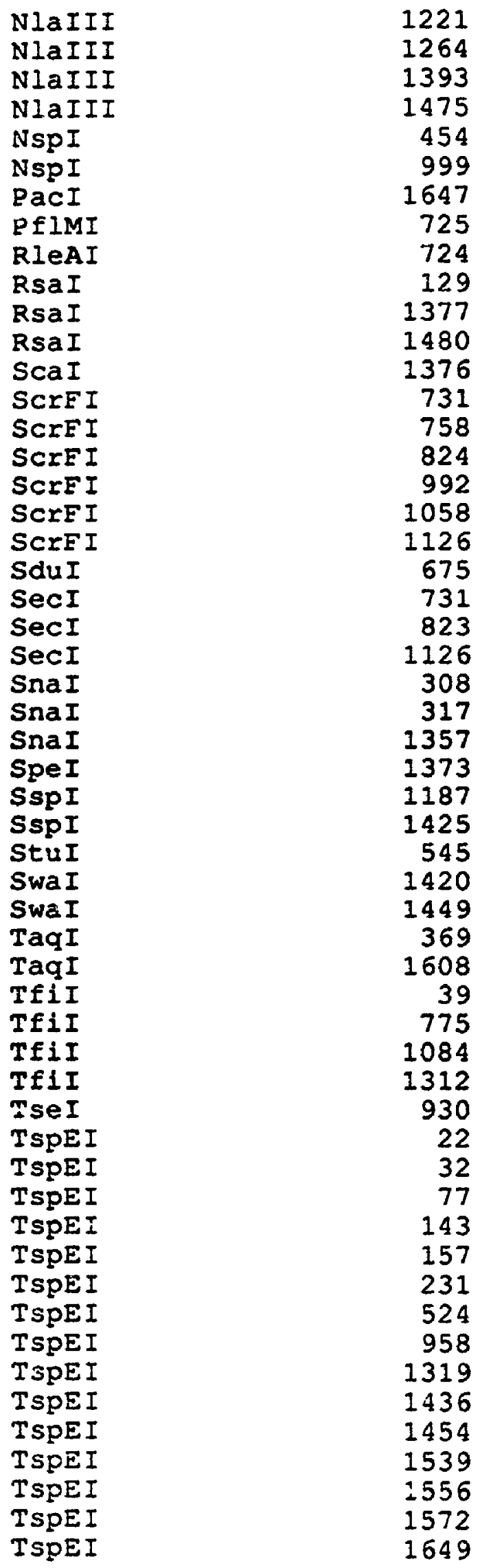


TSPEI

TSPEI

TSPEI

VspI

VspI

VspI

XhoII

XmnI
1667

1817

1869

92

1536

1818

1155

1070

The human interferon-alpha 17 gene restriction endonuclease recognition sites are summar:zed by enzyme.

\begin{tabular}{|c|c|c|}
\hline Name & Pattern & Sites \\
\hline AatII & gacgtc & 0 \\
\hline AcCI & gtmkac & 3 \\
\hline$k こ i I$ & $\operatorname{ccgc}$ & 0 \\
\hline ACII & aacgtt & 0 \\
\hline ACYI & grcgyc & 0 \\
\hline AEIII & cttaag & 0 \\
\hline AfIIII & acrygt & 1 \\
\hline AgeI & accggt & 0 \\
\hline AhaIII & tttaaa & 4 \\
\hline AluI & agct & 3 \\
\hline AlWNI & cagnnnctg & 2 \\
\hline ApaI & gggccc & 0 \\
\hline ApaBI & gcannnnntgc & 1 \\
\hline ApaI I & gtgcac & $\overline{0}$ \\
\hline ADOI & raatty & 7 \\
\hline AscI & ggcgcgce & 0 \\
\hline AsuI & ggnce & 2 \\
\hline AsuII & ttcgaa & 0 \\
\hline AvaI & cycgrg & 0 \\
\hline AvaII & ggwce & 0 \\
\hline AvaIII & atgcat & 0 \\
\hline AvIII & cctagg & 0 \\
\hline BaeI & acnnnngtayc & 0 \\
\hline BalI & tggcca & 0 \\
\hline BamHI & ggatcc & 0 \\
\hline BbvI & gcagc & 0 \\
\hline$B b \vee I I$ & gaagac & 1 \\
\hline BCCI & ccatc & 4 \\
\hline Bce83I & cttgag & 0 \\
\hline BcefI & $\operatorname{acggc}$ & 0 \\
\hline$B \subset g I$ & gcannnnnntcg & 0 \\
\hline$B C I I$ & tgatca & 1 \\
\hline BetI & wCcggw & 0 \\
\hline BgII & gccnnr!nnggo & 0 \\
\hline EgIII & agatct & 1 \\
\hline BinI & ggatc & 0 \\
\hline
\end{tabular}




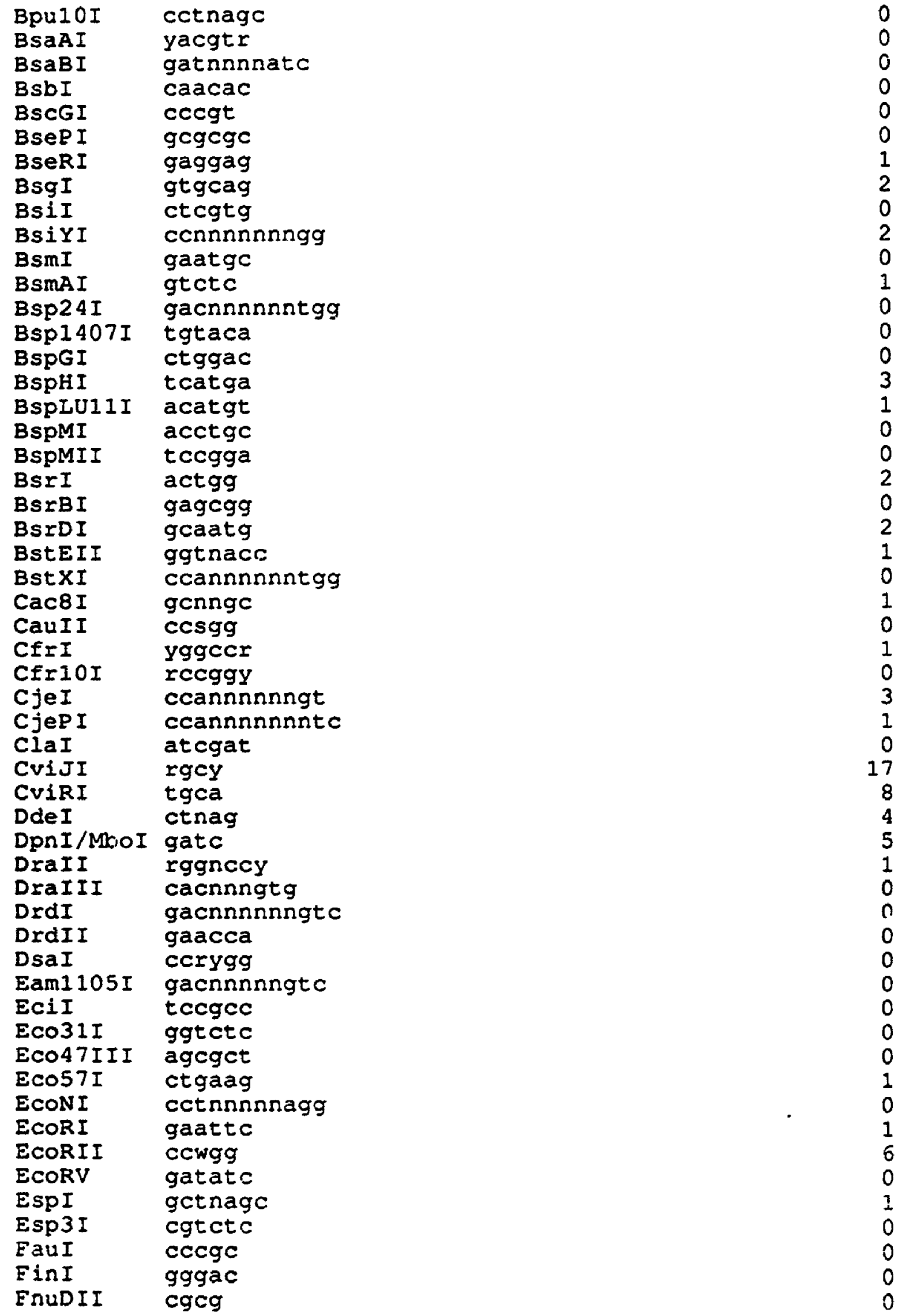




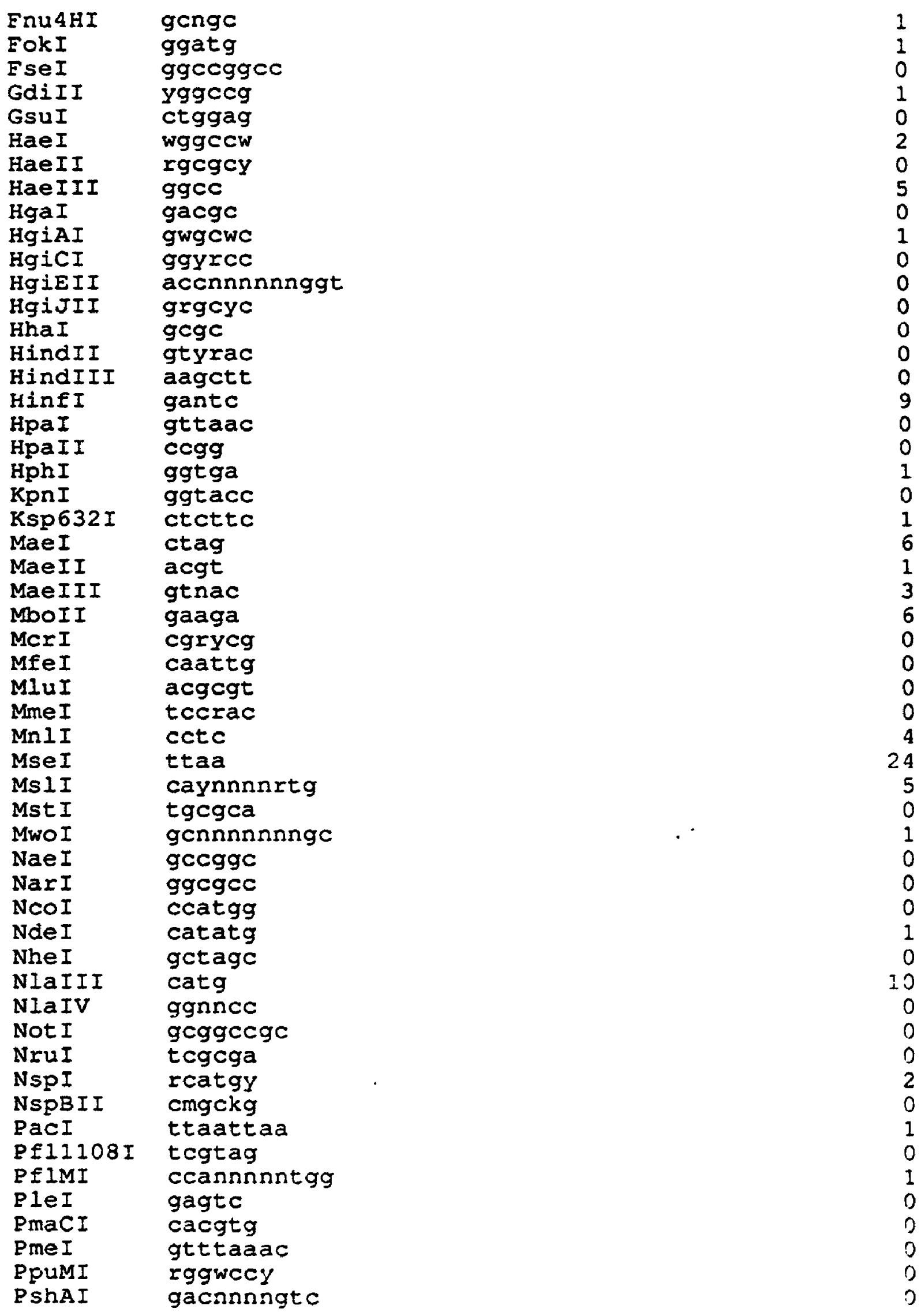




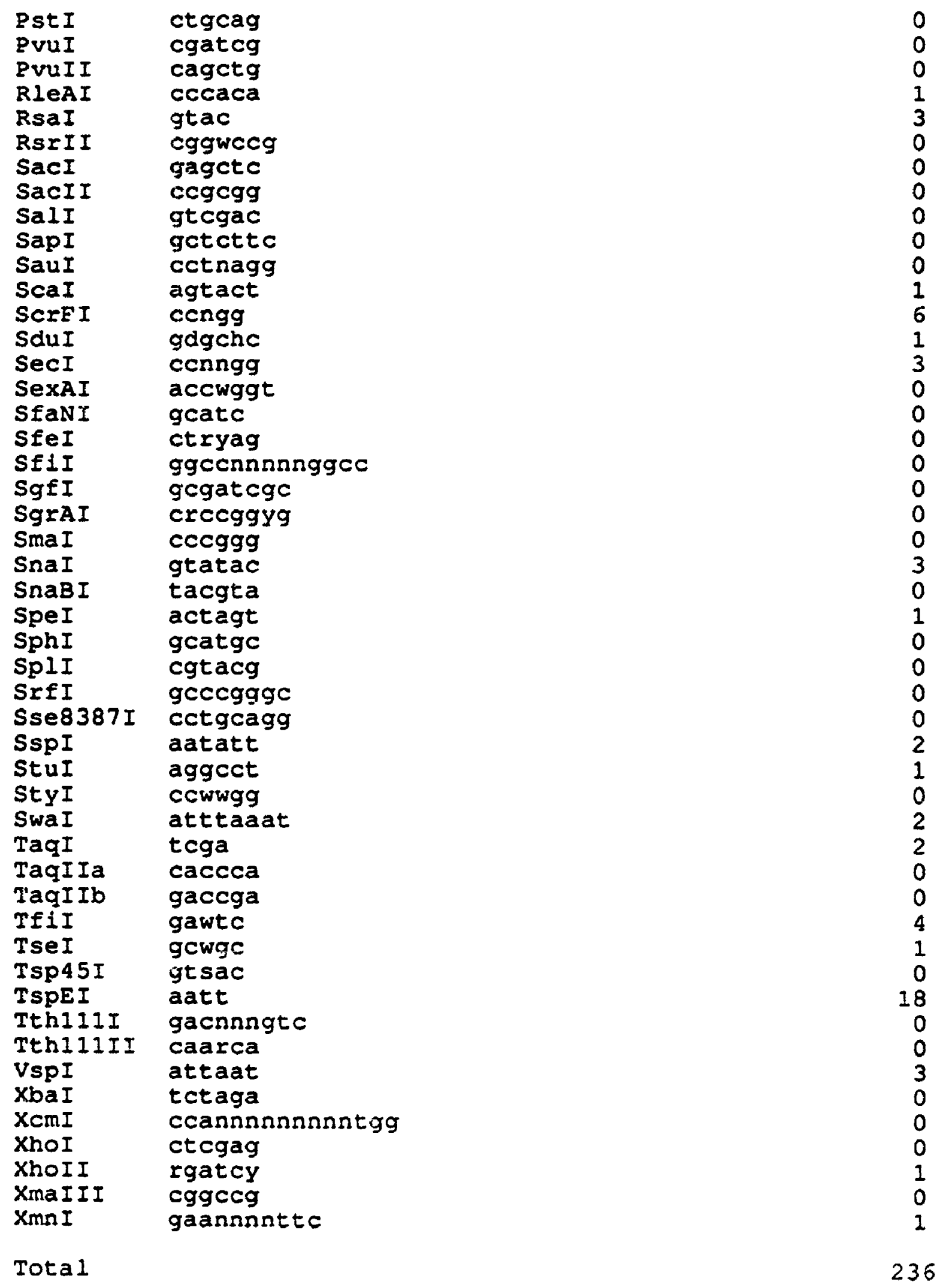




\section{Bibliography}

Adier, D. (1994). Human Chromosome 9 Ideogram, Department of Pathology, University of Washington.

Aho, A.V. (1990). "Algorithms for finding patterns in strings," in J. van Leeuwen ed.. Handbook of Theoretical Computer Science: Volume A Algorithms and Complexity, MIT Press, Cambridge, Massachusetts, pp. 256-300.

Aho, A.V. and Corasick, M.J. (1975). "Efficient string matching: an aid to bibliographic search," Communications of the Association for Computing Machinery 18 (6): 333-340.

Altschul, S.F., Boguski, M.S., Gish, W. and Wootton, J.C. (1994). "Issues in searching molecular sequence databases," Nature Genetics 6 (2): 119-129.

Anderson, J.E. (1990). "Restriction endonucleases and modification methylases," Current Opinion in Structural Biology 3 (1): 24-30.

Arrand, J.R., Myers, P.A. and Roberts, R.J. (1994). Unpublished observations.

Baeuerle, P.A. (1991). "The inducible transcription activator NF-KB: regulation by distinct protein subunits," Biochimica et Biophysira Acta 1072: 63-80.

Baeurele, P.A. and Baltimore, D. (1988). "I kappa B: a specific inhibitor of the NF kappa B transcription factor," Science 242: 540-546.

Baeza-Yates, R. and Regnier, M. (1990). "Fast algorithms for two dimensional and multiple pattern matching," in J.R. Gilbert and R. Karlsson eds., SWAT 90: Second Scandinavian Workshop on Algorithm Theory, Springer-Verlag Lecture Notes in Computer Science 447: 332-347.

Ballard, D.W., Walker, W.H., Doerre, S., Sista, P., Molitor, J.A., Dixon, E.P., Peffer, N.J., Hannink, M. and Greene, W.C. (1990). "The v-Rel oncogene encodes a KB enhancer binding protein that inhibits NF-KB function," Cell 63: 803-814. 
Bell, G.I. (1990). "Some computational challenges in the Human Genome Program," in C.R. Cantor and H.A. Lim eds., Electrophoresis, Supercomputing and the Human Genome, World Scientific Computing, pp. 6-11.

Benson, D., Lipman, D.J. and Ostell, J. (1993). "Genbank," Nucleic Acids Research 21 (13): 2963-2965.

Boguski, M.S. (1992). "Computational sequence analysis revisited: new databases, software tools and the research opportunities they engender," Journal of Lipid Restarch 33: 957-974.

Bookstein, R., Shew, J.Y., Chen, P.L., Scully, P. and Lee, W.H. (1990). "Suppression of tumorigenicity of human prostate carcinoma cells by replacing a mutated $\mathbf{R b}$ gene," Science 247: 712-715.

Bowcock, A.M., Farrer, L.A., Hebert, J.M., Bale, A.E. and Cavalli-Sforza, L. (1991). "A continuous linkage map of chromosome 13q with 39 distinct loci separated on average by 5.1 centimorgans," Genomics 11: $517-529$.

Bowden, D.W., Mao, J.I., Gill, T., Hsiao, K., Lillquist, J.S., Testa, D. and Vovis, G.F. (1984). "Cloning of eukaryotic genes in single-strand phage vectors: the human interferon genes," Gene 2'7: 87-99.

Boyer, R.S. and Moore, J.S. (1977). "A fast string searching algorithm," Communications of the Association for Computing Machinery 20: 762-772.

Brooks, J.E. (1987). "Properties and uses of restriction endonucleases," Methods in Enzymology 152: 113-129.

Buratowski. S. (1994). "The basics of basal transcription by RNA polymerase II," Cell 77: 1-3.

Chan, S.C., Wong, A.K.C. and Chiu, D.K.Y. (1992). "A survey of multiple sequence comparison methods," Bulletin of Mathematical Biology 54 (4): 563-598.

Collins, F.S. (1992). "Positional cloning: let's not call it reverse anymore," Nature Genetics 1 (1): 3-6. 
Collins, F. and Galas, D. (1993). "A new five-year plan for the US human genome project," Science 262 (5130): 43-46.

Commentz-Walter, B. (1979). "A string matching algorithm fast on the average," in H.A. Maurer ed., Automata Languages and Programming: Sixth Colloqium, SpringerVerlag Lecture Notes in Computer Science 71: 118-132.

Cuticchia, A.J., Fasman, K.H., Kingsbury, D.T., Robbins, R.J. and Pearson, P.L. (1993). "The GDB human genome data base anno 1993." Nucleic Acids Research 21 (13): 3003-3006.

Dayhoff, M.O., Schwartz, R.M. and Orcutt, B.C. (1978). "A model of evolutionary change in proteins," in M.O. Dayhoff ed. Atlas of Protein Sequence and Structure. National Biomedical Research Foundation, Washington, 5 (3): 345-352.

DeMaeyer, E. and DeMaeyer-Guignard, J. (1988). Interferons and Other Regulatory Cytokines, Wiley, New York.

Devereux, J., Haeberli, P. and Smithies, O. (1984). "A comprehensive set of sequence analysis programs for the VAX," Nucleic Acids Research 12 (1): 387-395.

Diaz, M.O., Bohlander, S. and Allen, G. (1993). "Nomenclature of the human interferon genes," Journal of Interferon Research 13: 243-244.

Diaz, M.O., Pomykala, H.M., Bohlander, S.K., Maltepe, E., Malik, K., Brownstein, B. and Olopade, O. (1994). "Structure of the human typ $\cdots 1$ interferon gene cluster determined from a YAC clone contig," Genomics 22: 540-552.

Dickerson, R.E. (1992). "DNA structure from A to Z," Methods in Enzymology 211: 67 111.

Dinter, H., Hauser, H., Mayr, U., Lammers, R., Bruns, W., Gross, G. and Collins, J. (1983). "Human interferon-beta and co-induced genes: molecular studies," in E. DeMaeyer and H. Schellekens eds. The Biology of the Interferon System. Elsevier, Amsterdam, pp. 33-44. 
Donis-Keller, H., Green, P., Helms, C., Cartinhour, S., Weiffenbach, B., Stephens, K., Keith, T.P., Bowden, D.W., Smith, D.R., Lander, E.S., Botstein, D., Akots, G., Rediker, K.S., Gravius, T., Brown, V.A., Rising, M.B., Parker, C., Powers, J.A., Watt, D.E., Kauffman, E.R., Bricker, A., Phipps, P., Muller-Kahle, H., Fulton, T.R., Ng, S., Schumm, J.W., Braman, J.C., Knowlton, R.G., Barker, D.F., Crooks, S.M., Linculn, S.E., Daly, M.J. and Abrahamson, J. (1987). "A genetic linkage map of the human genome," Cell 51: 319-337.

Dorr, R.T. (1993). "Inteferon- $\alpha$ in malignant and viral diseases," Drugs 45 (2): 177-211.

Drmanac, R., Strezoska, Z., Labal, I., Drmanac, S. and Crkvanjakov, R. (1990). "Laboratory methods: reliable hybridization of oligonucleotides as short as six nucleotides," DNA and Cell Biology 9: 527-534.

Dron, M., Rebouillat, D. and Tovey, M.G. (1992). "Tandem repeat polymers of a critical region of the human interferon- $\beta$ promoter exhibit a marked constitutive activity and enhanced responsiveness to transcriptional regulators in transfected HeLa cells," Journal of Interferon Research 12: 377-387.

Du, W. and Maniatis, T. (1992). “An ATF/CREB binding site protein is required for virus induction of the human interferon $\beta$ gene," Proceedings of the National Academy of Sciences USA 89: 2150-2154.

Dunn, J.M., Phillips, R.A., Zhu, X., Becker, A.J. and Gallie, B.L. (1989). "Mutations in the Rbl gene and their effects on transcription," Molecular and Cellular Biology 9: 4956-4604.

Engel, L.W. (1993). "The human genome project: history, goals and progress to date." Archives of Pathology and Laboratory Medicine 117 (5): 459-465.

Evans. S.W. and Whicher. J.T. (1993). "The cytokines: physiological and pathophysiological aspects," Advances in Clinical Chemistry 30: 1-88. 
Fan, C.M. and Maniatis, T. (1990). "A DNA-binding protein containing two widely separated zinc finger motifs that recognize the same DNA sequence," Genes and Development 4: 29-42.

Federation of European Biochemical Societies (FEBS) (1985). "Nomenclature for incompletely specified bases in nucleic acid sequences," European Journal of Biochemistry 150: 1-5.

Ford, G.M., Canton, M., Gallie, B.L., Phillips, R.A. and Becker, A.J. (1990). "A physical map around the retinoblastoma gene," Genomics 6: 284-292.

Franco, B., Guioli, S., Pragliola, A., Incerti, B., Bardoni, B., Tonlorenzi, R., Carrozzo, R., Maestrini. E., Pieretti, M., Taillon-Miller, P., Brown, C.J., Willard. H.F., Lawrence, C., Persico, M.G., Camerino, G. and Ballabio, A. (1991). "A gene deleted in Kallman's syndrome shares homology with neural cell adhesion and axonal path-firding molecules." Nature 353: 529-535.

Fraser, J.D., Irving, B.A., Crabtree, G.R. and Weiss, A. (1991). "Reguiation of interleukin-2 gene enhancer activity by the T cell accessory molecule CD28," Science 251: 313-316.

Friend, S.H., Bernards, R., Rogelj, S., Weinberg, R.A., Rapaport, J.M., Albert, D.M. and Dryja, T.P. (1986). "A human DNA segment with properties of the gene that predisposes to retinoblastoma and osteosarcoma," Nature 323: 643-646.

Fujita, T., Sakakibara, J., Sudo, Y., Miyamoto, M., Kimura, Y. and Taniguchi, T. (1988). "Evidence for a nuclear factor(s), IRF-1, mediating induction and silencing properties to human IFN- $\beta$ gene regulatory elements," European Molecular Biology Organization Journal 7: 3397-3405.

Furst, P., Hu, S., Harkett, R. and Hamer, D. (1988). "Conper activates methallothionein gene transcription by altering the conformation of a specific DNA binding protein," Cell 55: 705-717. 
Furukawa, Y., DeCaprio, J.A., Belvin, M. and Griffin, J.D. (1991). "Heterogeneous expression of the product of the retinoblastoma susceptibility gene in primary human leukemia cells," Oncogene 6: 1343-1346.

Gallie, B.L., Dunn, J.M., Hamel, P.A. and Phillips, R.A. (1990). "Point mutations in retinoblastoma," New England Journal of Medicine 322: 1397-1398.

Gallie, B.L., Ellsworth, R.M., Abramson, D.H. and Phillips, R.A. (1982).

"Retinoblastoma: spontaneous regression of retinoblastoma or benign manifestation of the mutation?" Eritish Joumal of Cancer 45: 513-521.

Garcia-Blanco, M.A., Clerc, R.G. and Sharp, P.A. (1989). "The DNA-binding homeo domain of the Oct-2 protein," Genes and Developinent 3: 739-745.

Genbank (1994). Personal communication.

Ghosh. D. (1993). "Status of the transcription factors database (TFD)," Nucleic Acids Research 21 (13): 3117-3118.

Giese, K., Cox, J. and Grosschedl, R. (1992). "The HMG domain of lymphoid enhancer factor 1 bends DNA and facilitates assembly of functional nucleoprotein structures," Cell 69: 185-195.

Gingeras, T.R., Greenough, L., Schildkraut, I. and Roberts, R.J. (1981). "Two new restriction endonucleases from Proteus vulgaris," Nucleic Acids Research 9 (18): 4525-4536.

Goeddel, D.V., Leung, D.W., Dull, T.J., Gross, M., Lawn, R.M., McCandliss, R., Seeburg, P.H., Ullich, A., Yelverton, E. and Gray, P.W. (1981). "The structure of eight distinct cloned human leukocyte interferon cDNAs," Nature 290: 20-26.

Goeddel, D.V., Yelverton, E., Ullrich, A., Heyneker, H.L., Miozzari, G., Holmes, W., Seeburg, P.H., Dull, T.J., May, L., Stebbing, N., Crea, R., Maeda. S., McCandliss, R., Sloma, A., Tabor, J.M., Gross. M., Familletti, P.C. and Pestka. S. (1980). "Human leukccyte interferon produced by E. coli is biologically active," Nature 287: 411-416. 
Goodbourn, S., Burstein, J. and Maniatis, T. (1986). "The human $\beta$-interferon gene enhancer is under negative control," Cell 45: 601-610.

Goodbourn, S., Zinn, K. and Maniatis, T. (1985). "Human $\beta$-interferon gene expression is regulated by an inducible enhancer element," Cell 41: 509-520.

Goodman, S.E. and Hedetniemi, S.T. (1977). Introduction to the Design and Analysis of Algorithms, McGraw-Hill, New York, pp. 265.

Goodrich, D.W. and Lee, W.H. (1993). "Molecular characterization of the retinoblastoma susceptibility gene," Biochimica et Biophysica Acta 155 (1): 43-61.

Gustafson, J.L., Montry, G.R. and Benner, R.E. (1988). "Development of parallel methods for a 1024-processor hypercube," SIAM Journal of Scientific and Statistical Computing 9 (4): 609-638.

Gutterman, J.U. (1994). "Cytokine therapeutics: lessons from interferon $\alpha$, Proceedings of the National Academy of Sciences USA 91: 1198-1205.

Guyer, M.S. and Collins, F.S. (1993). "The human genome project and the future of medicine," American Journal of Diseases of Children 147 (11): 1145-1152.

Hai, T., Liu, F., Coukos, W.J. and Green, M.R. (1989). "Transcription factor ATF cDNA clones: an extensive family of letcine zipper proteins able to selectively form DNA-binding heterodimers," Genes and Development 3: 2083-2090.

Harada, H., Fujita, T., Miyamoto, M., Kimura, Y., Maruyama, M., Furia, A., Miyata, T. and Taniguchi, T. (1989). "Structurally similar but functionally distinct factors, IRF-1 and IRF-2, bind to the same regulatory elements of IFN and IFN-inducible genes," Cell 58: 729-739.

Harbour, J.W., Lai, S.L., Whang, P.J., Gazdar, A.F., Mirna, J.D. and Kaye. F.J. (1988). "Abnormalities in structure and expression of the human retinoblastoma gene in SCLC." Science 241: 353-357. 
Hardy, K.J., Manger, B., Newton, M. and Stobo, J.D. (1987). "Molecular events involved in regulating human interferon- $\gamma$ gene expression during $T$ cell activation," Journal of Immunology 138: 2353.2358.

Hardy, K.J., Peterlin, B.M., Atchison, R.E. and Stobo, J.D. (1985). "Regulation of expression of the human interferon $\gamma$ gene," Proceedings of the National Academy of Sciences USA 82: 8173-8177.

Hatcker, P.J. and Quinn, M.J. (1991). Data-Parallel Programming, MIT Press, Cambridge, Massachusetts, pp. 6.

Hayes, M.P. and Zoon, K.C. (1994). "Production and action of interferons: new insights into molecular mechanisms of gene regulation and expression," Progress in Drug Research, in press.

Hedgpeth, J., Goodman, H.M. and Boyer, H.W. (1972). "DNA nucleotide sequence restricted by the RI enconuclease," Proceedings of the National Academy of Sciences USA 69 (11): 3448-3452.

Henco, K., Brosius, J., Fujisawa, A., Fujisawa, J.I., Haynes, J.R., Hochstadt, J., Kovacic, T., Pasek, M., Schamboeck, A., Schmid, J., Todokoro, K., Waelchli, M., Nagata, S. and Weissmann, C. (1985). "Structural relationship of human interferon alpha genes and pseudogenes," Journal of Molecular B1ology 185: 227-260.

Hiscott, J., Alper, D., Cohen, L., Leblanc, J.F., Sportza, L., Wong, A. and Xanthoudakis, S. (1989). "Induction of human interferon gene expression is associated with a nuclear factor that interacts with the NF-KB site of the human immunodeficiency virus enhancer," Journal of Virology 63 (6): 2557-2566. Hoffman, E.P. (1994). "The evolving genome project: current and future impact," American Joumal of Human Genetics 54 (1): 129-136. 
Horikoshi, M., Hai, T., Lin, Y.S., Green, M.R. and Roeder, R.G. (1988). "Transcription factor ATF interacts with the TATA box factor to facilitate establishment of a preinitiation complex," Cell 54: 1033-1042.

Horspool, R.N. (1980). "Pracical fast searching in strings," Software Practice and Experience 10: 501-506.

Horowitz, J.M., Yandeli, D.W., Park, S.H., Canning, S., Whyte, P., Buchkovich, K., Harlow, E., Weinberg, R.A. and Dryja, T.P. (1989). "Point mutational inactivation of the retinoblastoma antioncogene," Science 243: 936-940.

Houle, J.L. and Santoro, N. (1994). "Human interferon transcription factor recognition sequences," Technical Report, School of Computer Science, Carleton University, Ottawa, Canada.

Hu, Q.J., Dyson, N. and Harlow, E. (1990). "The regions of the retinoblastoma protein needed for binding to adenovirus E1A or SV40 large $\mathrm{T}$ antigen are common sites for mutations," European Molecular Biology Organization Journal 9: 1147-1155.

Intel Scientific Computers (1990). "Intel announces iPSC/860: 7.6 gflop system unveiled in London," iSCurrents, Winter 89/90: 1 .

Jacobson, K.B., Arlinghaus, H.F., Schmit, H.W., Sachleben, R.A., Brown, G.M., Thonnard, N., Sloop, F.V., Foote, R.S., Larimer, F.W., Woychik, R.P., England, M.W., Burchett, K.L. and Jacobson, D.A. (1991). "An approach to the use of stable isotopes for DNA sequencing," Genomics 9 (1): 51-59.

Kant, J.A. (1992). "DNA restriction enzymes and RFLPs in medicine," Methods in Biochemical Analysis 36: 129-149.

Keleher, C.... Gouthe, C. and Johnson, A.D. (1988). "The yeast cell-type specific repressor alpha 2 acts co-operatively with a non cell-type specific protein." Cell 53: 927-936. 
Kim, J.Y. and Shawe-Taylor, J. (1992). "Fast multiple keyword searching," in A. Apostolico. M. Crochemore, Z. Galil and U. Manber eds., Combinatorial Pattern Matching: Third Annual Symposium. Springer-Verlag Lecture Notes in Computer Science 644: 41-51.

King, C.T., Chou, W.H. and Ni, L.M. (1990). "Pipelined data-parallel algorithms: part I concept and modeling," IEEE Transactions on Parallel and Distributed Systems 1 (4): $470-485$.

Kornberg, A. and Baker, T.A. (1992). DNA Replication, Second Edition, W.H. Freeman, New York, pp. 4, 5, 7, 11.

Kuhl, D., de la Fuente, J., Chaturvedi, M., Parimoo, S., Ryals, J., Meyer, F. and Weissmann, C. (1987). "Reversible silencing of enhancers by sequences derived from the human IFN- $\alpha$ promoter," Cell 50: 1057-1069.

Kumar, V. and Gupta, A. (1994). "Analyzing scalabiitity of parallel algorithms and architectures," Journal of Parallel and Distributed Computing 22: 379-391.

Labdon, J.E., Gibson, K.D., Sun, S. and Pestka, S. (1984). "Some species of human leukocyte interferon are glycosylated," Archives of Biochemistry and Biophysics 232: $422-426$.

Lai, M.H.T. and Joklik, K. (1973). "The induction of interferon by temperature-sensitive mutants of reovirus, UV-irradiated reovirus and subviral reovirus particles," Virology 51: 191-204.

Latchman, D.S. (1993). "Transcription factors: an overview," International Journal of Experimental Pathology 74: 417-422.

Lawn, R.M., Adelman, J.P., Dull, T.J., Gross, M., Goeddel, D. and Ullrich, A. (1981a). "DNA sequence of two closely linked human leukocyte interferon genes," Science 212: 1159-1162. 
Lawn, R.M., Gross, Mi, Houck, C.M., Franke, A.E., Gray, P.V. and Goeddel, D.V. (1981b). "DNA sequence of a major human leukocyte interferon gene,"

Proceedings of the National Academy of Sciences USA 78: 5435-5439.

Lee, W.H., Bookstein, R., Hong, F., Young, L.J., Shew, J.Y. and Lee, E.Y.H.P. (1987a). "Human retinoblastoma susceptibility gene: cloning, identification and sequence," Science 235: 1394-1399.

Lee, W.H., Shew, J.Y., Hong, F.D., Sery, T.W., Donoso, L.A., Young, L.J., Bookstein, R. and Lee, E.Y.H.P. (1987b). "The retinoblastoma susceptibility gene encodes a nuclear phosphoprotein associated with DNA binding activity," Nature 329: 642-645.

Lee, E.Y., To, H., Shew, J.Y., Bookstein, R., Scully, P. and Lee, W.H. (1988). "Inactivation of the retinoblastoma susceptibility gene in human breast cancers," Science 241: 218-221.

Legouis, R., Hardelin, J.P., Levilliers, J., Claverie, J.M., Compain, S., Wunderie, V., Millasseau, P., Le Paslier, D., Cohen, D., Caterina, D., Bougueleret, L., Delemarre-Van de Waal, H., Lutfalla, G., Weissenbach, J. and Petit, C. (1991). "The candidate gene for the $\mathrm{X}$-linked Kallmann syndrome encodes a protein related to adhesion inolecules," Cell 67: 423-435.

Lehninger, A.L. (1982). Principles of Biochemistry, Worth, New York, pp. 56, 57.

Lenardo, M.J. and Baltimore, D. (1989). "NF-kB: a pleiotropic mediator of inducible and tissue-specific gene control," Cell 58: 227-229.

Levine, A.J. (1993). "The tumor suppressor genes," Annual Review of Biochemistry 62: 623-651.

Lindsay, S.M. and Philipp, M. (1991). "Can the scanning tunneling microscope sequence DNA?" Genetic Analysis 8: 8-13. 
MacDonald, N.J., Kuhl, D., Maguire, D., Naf, D., Gallant, P., Goswamy, A., Hug, H., Bueler, H., Chaturvedii, M., de la Fuente, J., Ruffner, H., Meyer, F. and Weissmann, C. (1990). "Different pathways mediate virus inducibility of the human IFN- $\alpha 1$ and IFN- $\beta$ genes," Cell 60: 767-779.

Macelis, D. (1994). Personal communication.

Maeda, S., McCandliss, R., Gross, M., Sloma, A., Familletti, P.C., Tabor, J.M., Evinger, M., Levy, W.P. and Pestka, S. (1980). "Construction and identification of bacterial plasmids containing nucleotide sequence for human leukocyte interferon," Proceedings of the National Academy of Sciences USA 77: 70107013.

Maniatis, T. (1988). "Mechanisms of human $\beta$-interferon gene regulation," Harvey Lectures 82: 71-104.

Maniatis, T., Whittemore, L.A., Wei, D., Fan, C.M., Keller, A.D., Palombella, V.J. and Thanos, D.N. (1992). "Positive and negative control of human interferon- $\beta$ gene expression," in S.L. McKnigitt and K.R. Yamamoto eds., Transcriptional Regulation, Cold Spring Harbor Laboratory, Cold Spring Harbor, New York, pp. 1193-1220.

Mantei, N., Schwarzstein, M., Streuli, M., Panem, S., Nagata, S. and Weissmann, C. (1980). "The nucleotide sequence of a cloned human leukocyte interferon cDNA," Gene 10: 1-10.

Matthews, C.R. (1993). "Pathways of protein folding," Annual Review of Biochemistry 62: 653-683.

McKusick, V.A. (1994). Mendelian Inheritance in Man: A Catalog of truman Genes and Genetic Disorders, 11 th. ed., John Hopkins University Press, Baltimore. 
Meitinger, T., Heye, B., Petit, C., Levilliers, J., Golla, A., Moraine, C., Dalla Piccola, B., Sipfrell, W.G., Murken, J. and Ballabio, A. (1990). "Definitive localization of $\mathrm{X}$-linked Kallman syndrome (hypogonadotropic hypogonadism and anosmia) to Xp22.3: clone linkage to the hypervariable repeat sequence CRI-S232," American Journal of Human Genetics 47: 664-669.

Miyamoto, M., Fujita, T., Kimura, Y., Maruyama, M., Harada, H., Sudo, Y., Miyata, T. and Taniguchi, T. (1988). "Regulated expression of a gene encoding a nuclear factor, IRF-1, that specifically binds to IFN- $\beta$ gene regulatory elements," Cell 54: 903-913.

Morimoto, R.I. (1992). "Transcription factors: positive and negative regulators of ce.l growth and disease," Current Opinion in Cell Biology 4 (3): 480-487.

Mount, D.W. and Conrad, B. (1986). "Improved programs for DNA and protein sequence analysis on the IBM personal computer and other standard computer systems," Nucleic Acids Research 14 (1): 443-454.

Murgolo, N.J., Windsor, W.T., Hruza, A., Reichert, P., Tsarbopoulos, A., Baldwin, S., Huang, E., Pramanik, B., Ealick, S. and Trotta, P.P. (1993). "A homology model of human interferon $\alpha-2$," Proteins: Structure, Function and Genetics 17: 62-74.

Murphy, K.P. and Freire, E. (1992). "Thermodynamics of structural stability and cooperative folding behavior in proteins," Advances in Protein Chemistry 43: 313361.

Naf, D., Hardin, S.E. and Weissmann, C. (1991). "Multimerization of AAGTGA and GAAAGT generates sequences that mediate virus inducibility by mimicking an interferon promoter element," Proceedings of the National Academy of Sciences USA 88: 1369-1373.

Nagata, S., Mantei, N. and Weissmann, C. (1980). "The structure of one of the eight or more distinct chromosomal genes for human interferon-alpha," Nature 287: 401408. 
Nakayama, K., Shimizu, H., Mitomo, K., Watanabe, T., Okamoto, S.I. and Yamamoto, K.I. (1992). "A lymphoid cell-specific nuclear factor containing c-Rel-like proteins preferentially interacts with interleukin-6 kB-related motifs whose activities are repressed in lymphoid cells," Molecular and Cellular Biology 12 (4): 1736-1746.

Nourbakhsh, M., Hoffmann, K. and Hauser, H. (1993). "Interferon- $\beta$ promoters contain a DNA element that acts as a position-independent silencer on the NF- $\mathrm{KB}$ site," EMBO Journal 12 (2): 451-459.

Olson, M.V. (1993). "The human genome project," Proceedings of the National Academy of Sciences USA 90: 4338-4344.

Olson, M., Hood, L., Cantor, C. and Botstein, D. (1989). "A common language for physical mapping of the human genome," Science 245: 1434-1435.

Pabo, C.O. and Sauer, R.T. (1992). "Transcription factors: structural families and principles of DNA recognition," Annual Review of Biochemistry 61: 1053-1095.

Pearson, M.L. and Soll, D. (1991). "The Human Genome Project: a paradigm for information management in the life sciences," FASEB Journal 5 (1): 35-39.

Penix, L., Weaver, W.M., Pang, Y., Young, H.A. and Wilson, C.B. (1993). "Two essential regulatory elements in the human interferon $\gamma$ promoter confer activation specific expression in T cells," Journal of Experimental Medicine 178: 1483-1496.

Ponder, J.W. and Richards, F.M. (1987). "Tertiary templates for proteins. Use of packing criteria in the enumeration of allowed sequences for different structural classes," Journal of Molecular Biology 193: 775-791.

Prestridge, D.S. and Stormo, G. (1993). "Signal Scan 3.0: new database and program features," Computer Applications in the Biological Sciences 9 (1): 113-115. Ptashne. M. (1988). "How eukaryotic transcriptional activators work," Nature 335: 683689. 
Quinn, M.J. and Hatcher, P.J. (1990). "Data-parallel programming on multicomputers," IEEE Software 7 (5): 69-76.

Quinn, M.J. (1994). Parallel Computing: Theory and Practice 2nd. ed., McGraw-Hill, New York.

Ranka, S and Sahni, S. (1990). Hypercube Algorithms with Applications to Image Processing and Pattern Recognition, Springer-Verlag, New York.

Richardson, J.S. and Richardson, D.C. (1989). "Principles and patterns of protein conformation," in G.D. Fasman ed., Prediction of Protein Structure and the Principles of Protein Conformation, Plenum Press, New York, pp. 1-98.

Rizo, J. and Gierasch, L.M. (1992). "Constrained pe ytides: models of bioactive peptides and protein substructures," Annual Review of Biochemistry 61: 387-418.

Roberts, R.J. and Halford, S.E. (1993). "Type II restriction endonucleases," in S.M. Linn, R.S. Lloyd and R.J. Roberts eds., Nucleases 2nd ed., Cold Spring Harbor Laboratory Press, Cold Spring Harbor, New York.

Roberts, R.J. and Macelis, D. (1994). "REBASE - restriction enzymes and methylases," Nucleic Acids Research 22 (17): 3628-3639.

Ryals, J., Dierks, P., Ragg, H. and Weissmann, C. (1985). “A 46-nucleotide promoter segment from an IFN- $\alpha$ gene renders an unrelated promoter inducible by virus," Cell 41: 497-507.

Saad, Y. and Schultz, M.H. (1988). "Topological properties of hypercubes," IEEE Transactions on Computers 37: 867-872.

Sakai, T., Ohtani, N., McGee, T.L., Robbins, P.D. and Dryja, T.P. (1991). "Oncogenic germ-line mutations in Spl and ATF sites in the human retinoblastoma gene." Nature 353: 83-86.

Scheidereit, C., Heguy, A. and Roeder, R.G. (1987). "Identification and purification of a human lymphoid-specitic octamer-binding protein (OTF-2) that activattes transcription of an imnunoglobulin promoter in vitro," Cell 51: 783-793. 
Schwarz, E.M., Salgame, P. and Bloom, B.R. (1993). "Molecular regulation of human interleukin 2 and T-cell function by interleukin 4," Proceedings of the National Academy of Sciences USA 90: 7734-7738.

Sehgal, P.B. (1982). "The interferon genes," Biochemica et Biophysica Acta 695: 17-33.

Sen, G.C. and Lengyel, P. (1992). "The interferon system: a bird's eye view of its biochemistry," Journal of Biological Chemistry 267: 5017-5020.

Sgaramella, V. (1972). "Enzymatic oligomerization of Bacteriophage P22 DNA and of linear Simian Virus 40 DNA," Proceedings of the National Academy of Sciences USA 69: 3389-3393.

Shields, J.A. (1983). Diagnosis and Management of Intraocular Tumors, C.V. Mosby, St. Louis, pp. 437-438.

Sica, A., Tan, T.H., Rice, N., Kretzschmar, Mi., Ghosh, P. and Young, H.A. (1992). "The c-Rel protooncogene product c-Rel but not NF-KB binds to the intronic region of the human interferon- $\gamma$ gene at a site related to an interferon-stimulable response element," Proceedings of the National Academy of Sciences USA 89: 1740-1744.

Singleton, P. and Sainsbury, D. (1987). Dictionary of Microbiology and Molecular Biology, 2nd. ed., Wiley, New York, pp. 383-384.

Smith, D.W. (1994). Biocomputing: Informatics and Genome Projects, Academic Press, San Diego.

Smith, H.O. and Nathans, D. (1973). "A suggested nomenclature for bacterial host modification and restriction systems and their enzymes," Journal of Molecular Biology 81: 419-423.

Smith, R. (1988). "A finite state machine algorithm for finding restriction sites and other pattern matching applications," Computer Applications in the Biological Sciences 4 (4): $459-465$. 
Soper, S.A., Davis, L.M., Fairfield, F.R., Hammond, M.L., Harger, C.A., Jett, J.H.. Keller, R.A., Marrone, B.L., Martin, J.C., Nutter, H.L., Shera, E.B. and Simpson, D.J. (1991). "Rapid sequencing of DNA sequencing based on single molecule detection," Proceedings of the Society for Photo-optical Instrumentation Engineers 1435: 168-178.

Staden, R. (1988). "Methods to define and locate patterns of motifs in sequences," Computer Applications in the Biosciences 4: 53-60.

Staden, R. (1990). "Searching for patterns in protein and nucleic acid sequences," Methods in Enzymology 183: 193-211.

Squire, J. and Palais, S.M. (1963). "Programming and design considerations of a highly parallel computer," in Proceedings of the AFIP Spring Joint Computer Conference 23: $395-400$.

Streuli, M., Nagata, S. and Weissmann, C. (1980). "At least three human type alpha interferons: structure of alpha-2," Science 209: 1343-1347.

Sullivan, H. and Bashkow, T. (1977). "A large scale, homogeneous, fully distributed parallel machine," in Proceedings of the Fourth Symposium on Computer Architecture, pp. 105-117.

Suzuki, D.T., Griffiths, A.J.F., Miller, J.H. and Lewontin, R.C. (1989). An Introduction to Genetic Analysis, Fourth Edition, W.H. Freeman, New York, pp. 262, 263, 267, 339.

Tanaka, N., Kawakami, T. and Taniguchi, T. (1993). "Recognition DNA sequences of interferon regulatory factor 1 (IRF-1) and IRF-2, regulators of cell growth and the interferon system," Molecular and Cellular Biology 13 (8): $4531-4538$.

Tanaka, N. and Taniguchi, T. (1992). "Cytokine gene regulation: regulatory cis-elements and DNA binding factors involved in the interferon system," Advances in Immunology 52: 263-281. 
Taniguchi, T. (1989). "Regulation of interferon- $\beta$ gene: structure and function of cis-elements and trans-acting factors," Journal of Interferon Research 9: 633-640.

Todokoro, K., Kioussis, D. and Weissmann, C. (1984). "Two non-allelic human interferon alpha genes with ideni_cal coding regions," European Molecular Biology Organization Journal 3: 1809-1812.

Toguchida, J., McGee, T.L., Paterson, J.C., Eagle, J.R., Tucker, S., Yandell, D.W. and Dryja, T.P. (1993). "Complete genomic sequence of the human retinoblastoma susceptibility gene," Genomics 17: 535-543.

Treacey, M.N., He, X. and Rosenfeld, M.G. (1991). "I-POU: a POU domain protein that inhibits neuron-specific gene activation," Nature 350: 577-584.

Trent, J.M., Olson, S. and Lawn, R.M. (1982). "Chromosomal localization of human leukocyte, fibroblast and immune interferon genes by means of in situ hybridization," Proceedings of the National Academy of Sciences USA 79: 7809-7813.

Wagner, R.P., Maguire, M.P. and Stallings, R.L. (1993). Chromosomes: A Synthesis, Wiley-Liss, New York.

Wang, T.C. and Brand, S.J. (1990). "Islet cell-specific regulatory domain in the gastrin promoter contains adjacent positive and negative DNA elements," Journal of Biological Chemistry 265: 8908-8914.

Watson, J.D., Hopkins, N.H., Roberts, J.W., Steitz, J.A. and Weiner, A.M. (1987). Molecular Biology of the Gene, Fourth Edition, Benjamin Cummings, Menlo Park, California, pp. 42, 44.

Weidle, U. and Weissmann, C. (1983). "The 5'-flanking region of a human IFN- $\alpha$ gene mediates viral induction of transcription," Nature 303: 442-446.

Weinberg, R.A. (1992). "The retinoblastoma gene and gene product," in A.J. Levine ed., Cancer Surveys Volume 12 Tumor Suppressor Genes the Cell Cycle and Cancer, pp. 5-24. 
W'einmann, R. (1992). "The basic RNA polymerase II transcriptional machinery," Gene Expression 2 (2): 81-91.

Weissmann, C. and Weber, H. (1986). "The interferon genes," Progress in Nucleic Acid Research 33: 251-300.

Wetzel, R. (1981). "Assignment of the disulfide bonds of leukocyte interferon." Nature 289: 606-60̄

Wilson, G.G. and Murray, N.E. (1991). "Restriction and modification systems," Annual Review of Genetics 25: 585-627.

Wingender, E., Heinemeyer, T. and Lincoln, D. (1990). "Regulatory DNA sequences: predictability of their function," in J. Collins and A.J. Driesel eds., Genome Analysis: From Sequence to Function, 1st European Meeting 1990 Human Genome Organisation (HUGO), Huthig Buch Verlag, Heidelberg, pp. 95-108.

Yager, T.D., Nickerson, D.A. and Hood, L.E. (1991). " the human genome project: creating an infrastructure for biology and medicine," Trends in Biochemical Sciences 16 (12): 454-461.

Yamamoto, K.R., Gonzalez, G.A., Menzel, P., Rivier, J. and Montminy, M.R. (1990). "Characterization of a bipartite activation domain in transcription factor CREB." Cell 60: 611-617.

Yuan, R. (1981). "Structure and mechanism of multifunctional restriction endonucleases," Annual Review of Biochemistry 50: 285-315.

Zacksenhaus, E., Bremner, R., Jiang, Z., Gill, R.M., Muncaster, M., Sopta, M., Phillips, R.A. and Gallie, B.L. (1993). "Unraveling the function of the retinoblastoma gene," Advances in Cancer Research 61: 115-141.

Zhang, K., Wang, M.X., Munier, F., Roth, D., Mastrangelo, D., Chung, S., Shields. J.A. and Donoso, L.A. (1993). "Molecular genetics of retinoblastoma," International Ophtalmology Clinics 33 (3): 53-65. 
Zimmerman, S.B. (1982). "The three-dimensional structure of DNA," Annual Review of Biochemistry 51: 395-427.

Zoon, K.C. (1987). "Human interferons: structure and function," in I. Gresser ed. Interferon 9, Academic Press, New York. 

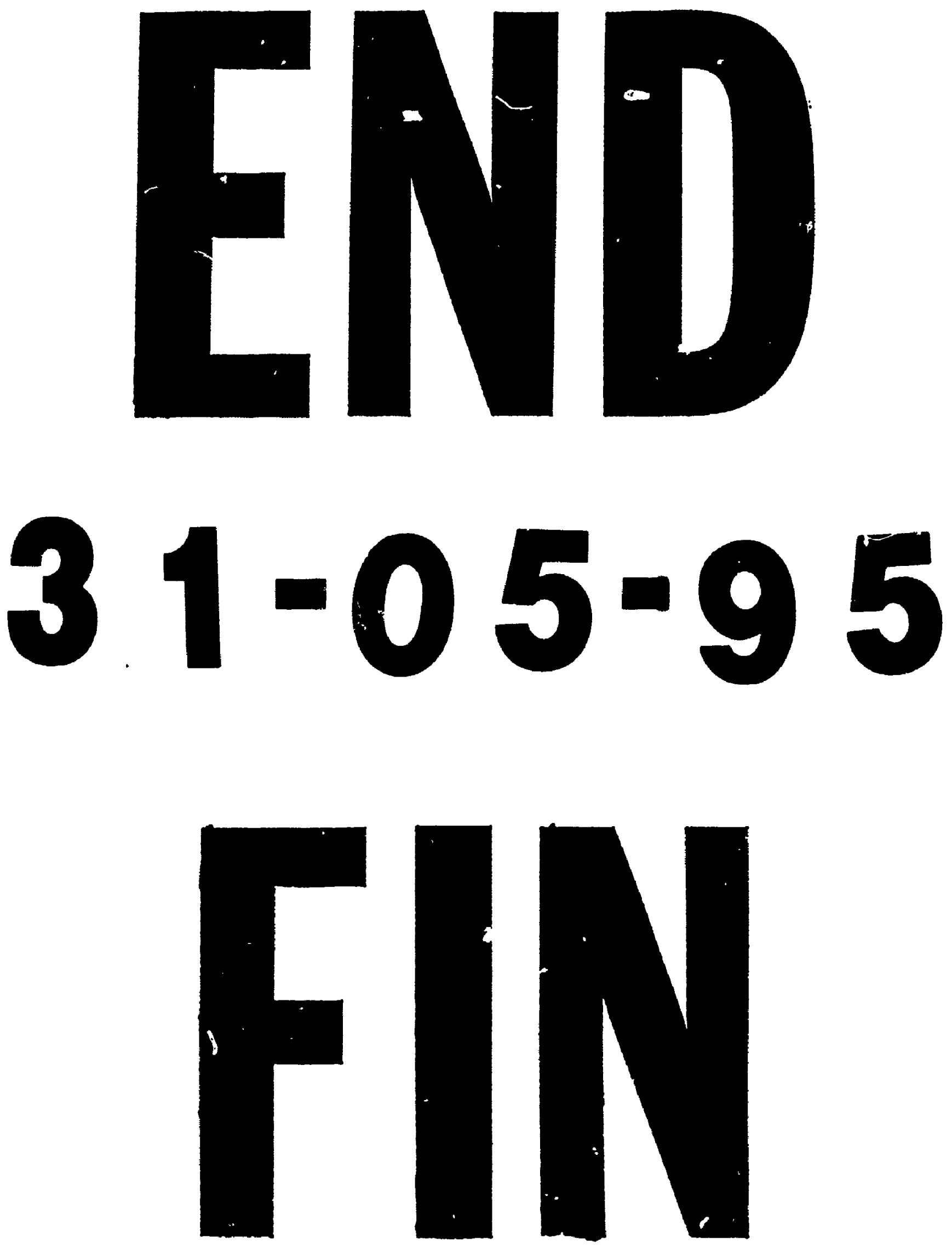


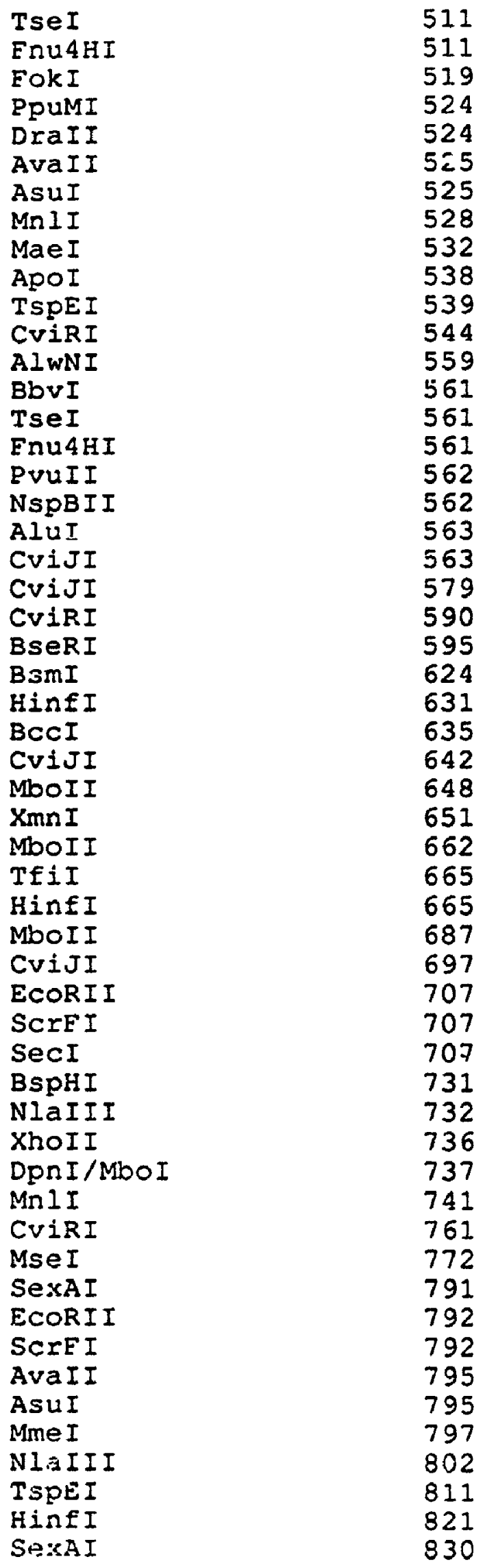

